Merci d'utiliser le titre suivant quand vous citez ce document :

Liebig, T. et al. (2012),"L'intégration des immigrés et de leurs enfants sur le marché du travail en Suisse", Documents de travail de l'OCDE sur les affaires sociales, l'emploi et les migrations, No.128, Éditions de l'OCDE

Documents de travail de l'OCDE sur les affaires sociales, l'emploi et les migrations

\title{
L'intégration des immigrés et de leurs enfants sur le marché du travail en Suisse
}

Thomas Liebig, Sebastian Kohls ef Karolin Krause

128

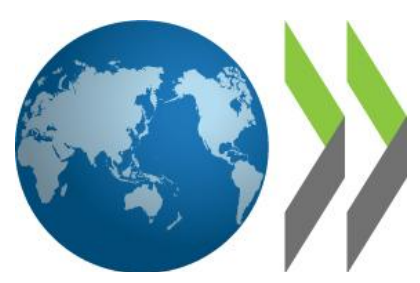


Organisation de Coopération et de Développement Économiques

Organisation for Economic Co-operation and Development

02-Feb-2012

DIRECTION DE L'EMPLOI, DU TRAVAIL ET DES AFFAIRES SOCIALES

Français - Or. Anglais

COMITE DE L'EMPLOI, DU TRAVAIL ET DES AFFAIRES SOCIALES

DOCUMENTS DE TRAVAIL DE L'OCDE SUR LES AFFAIRES SOCIALES,

L'EMPLOI ET LES MIGRATIONS, ${ }^{\circ} 128$

L'INTÉGRATION DES IMMIGRÉS ET DE LEURS ENFANTS SUR
LE MARCHÉ DU TRAVAIL EN SUISSE

Thomas Liebig, Sebastian Kohls et Karolin Krause

$J 13, J 15, J 21, J 24, J 61, J 68, J 7, J 8$

La traduction en allemand et en italien du résumé, ainsi que de l'évaluation et des recommandations sont dans les annexes 2 et 3 , respectivement

"Ce document et toute carte qu'il peut comprendre sont sans préjudice du statut de tout territoire, de la souveraineté s'exerçant sur ce dernier, du tracé des frontières et limites internationales, et du nom de tout territoire, ville ou région. »

\section{JT03315264}


DELSA/ELSA/WD/SEM(2012)2

\title{
DIRECTION DE L'EMPLOI, DU TRAVAIL ET DES AFFAIRES SOCIALES
}

www.oecd.org/els-fr

\section{DOCUMENTS DE TRAVAIL DE L'OCDE : QUESTIONS SOCIALES, EMPLOI ET MIGRATIONS}

www.oecd.org/els/documentsdetravail

Le but de cette série de documents est de fournir à un plus grand nombre de lecteurs des études sur les politiques sociales, la migration et le marché du travail destinées à l'origine à un usage interne à l'OCDE. En général, ces études ont un caractère collectif, et seuls les auteurs principaux sont cités nommément. Les documents ne sont généralement disponibles que dans leur langue d'origine - anglais ou français - avec un résumé dans l'autre langue.

Les commentaires sont les bienvenus, et sont à envoyer à la Direction de l'emploi, du travail et des affaires sociales, 2, rue André-Pascal, 75775 PARIS CEDEX 16, France. Des exemplaires supplémentaires, en nombre limité, sont disponibles sur demande.

Les opinions exprimées et les arguments employés dans ce document sont la responsabilité des auteurs, et ne représentent pas nécessairement ceux de l'OCDE.

Les demandes de reproduction ou de traduction totales ou partielles doivent être adressées à :

\author{
M. le Chef du Service des Publications \\ OCDE \\ 2, rue André-Pascal \\ 75775 Paris, Cedex 16 \\ France
}

Copyright OCDE 2010 


\section{REMERCIEMENTS}

Ce rapport a été rédigé par la Division des migrations internationales de l'OCDE sous la supervision de Thomas Liebig. Le rapport a été écrit conjointement avec Sebastian Kohls et Karolin Krause. Yassine Khoudja a apporté une aide statistique précieuse. Le rapport contient également une contribution d'Etienne Piguet (Université de Neuchâtel).

Les auteurs sont reconnaissants pour le soutien et les conseils qu'ils ont reçus de Jonathan Chaloff, Jean-Christophe Dumont, Jean-Pierre Garson, Georges Lemaître, John Martin, Stefano Scarpetta and Alfonso Sousa-Poza.

Un projet de rapport a été présenté et discuté à l'occasion de la Réunion du Comité de l'OCDE de l'Emploi, du Travail et des Affaires sociales le 14 octobre 2011. Les auteurs remercient les participants du Comité pour leurs précieux commentaires.

La préparation de ce rapport n'aurait pu se faire sans le soutien des Autorités suisses, en particulier Kurt Rohner et Adrian Gerber et leurs équipes respectives de l'Office fédéral des migrations. Les auteurs sont également reconnaissants à l'Office statistique suisse et, en particulier, à Alain Vuille et son équipe, ainsi qu'à Sandra Hupka-Brunner et Thomas Meyer de l'Université de Bâle, pour leur coopération et pour toutes les données qu'ils leur ont fournies. Enfin, les auteurs tiennent à remercier tous les acteurs rencontrés durant les missions en Suisse, pour les précieuses informations récoltées et les discussions particulièrement fructueuses.

\section{Contact:}

Thomas Liebig

Division des migrations internationales

Direction de l'Emploi, du Travail et des Affaires sociales

2, rue André-Pascal

F-75775 Paris Cedex 16

Tel. +33-1-45 249068

Thomas.Liebig@oecd.org

www.oecd.org/migration 


\section{RÉSUMÉ}

La Suisse est, parmi les pays de l'OCDE, l'un de ceux où il y a la plus forte proportion d'immigrés $27 \%$ des personnes en âge de travailler sont nées à l'étranger - et la question de l'immigration occupe une place importante, tant dans l'agenda politique que dans le débat public. Compte tenu des nombreux débats que la question suscite dans le pays, on pourrait être tenté de penser que les immigrés sont moins bien intégrés en Suisse que dans d'autres pays.

Les faits montrent cependant que, dans l'ensemble, l'intégration fonctionne plutôt bien en Suisse. La situation sur le marché du travail de la population immigrée dans son ensemble est largement favorable au regard de ce qu'on observe dans d'autres pays. Pour les hommes comme pour les femmes, le taux d'emploi des immigrés est plus élevé que dans les autres pays de l'OCDE.

Ces bons résultats s'expliquent principalement par la situation, globalement bonne, du marché du travail en Suisse, et par une combinaison particulière de pays d'origine. La majeure partie des migrants (plus de $60 \%$ ) sont originaires de pays de l'OCDE à haut revenu, et plus de la moitié d'entre eux viennent des pays voisins dont les langues nationales sont les mêmes que celles de la Suisse. Les autres immigrés proviennent majoritairement des pays issus de l'ex-Yougoslavie et de la Turquie.

Au cours des dernières années, suite à l'introduction progressive de la libre circulation avec les pays membres de l'Union européenne, la Suisse a vu arriver un nombre exceptionnellement élevé d'étrangers. Quelque $5 \%$ de la population résidante se compose d'immigrés récents, c'est-à-dire installés depuis moins de cinq ans. Les nouveaux arrivants sont, pour la plupart, originaires de pays voisins, en particulier d'Allemagne, et ils s'intègrent bien sur le marché du travail au regard de tous les indicateurs conventionnels.

Cependant, malgré un tableau globalement positif, la situation est plus délicate pour certaines catégories d'immigrés, par exemple les femmes qui ont de jeunes enfants. Peu d'initiatives sont prises pour favoriser l'intégration des femmes immigrées, qui n'ont généralement pas accès à tous les volets de la politique active du marché du travail. Certains éléments semblent indiquer, par ailleurs, que le taux d'activité de ce groupe de population est en baisse depuis quelques années.

Un autre groupe enregistre des taux d'emploi médiocres, y compris dans les comparaisons internationales : il s'agit des migrants humanitaires récemment arrivés, qui semblent avoir plus de mal que les cohortes précédentes à s'intégrer sur le marché du travail en Suisse. Contrairement à d'autres pays, la Suisse n'a pas encore mis en place un programme d'intégration spécifique pour cette catégorie de nouveaux arrivants, ce qui peut expliquer en partie leurs mauvaises performances. Au vu des expériences encourageantes enregistrées par les pays de l'OCDE proposant des programmes d'intégration structurés et axés sur le marché du travail, il serait souhaitable que la Suisse envisage sérieusement l'adoption d'initiatives similaires.

Le taux d'emploi généralement élevé des immigrés en Suisse est également lié à une fréquente surqualification des migrants diplômés de pays non membres de l'OCDE. Les qualifications acquises à l'étranger sont insuffisamment valorisées sur le marché du travail suisse, et il n'y a que peu de cours passerelle. Contrairement à d'autres pays de l'OCDE, la Suisse a mis en place peu de programmes de parrainage ou d'initiatives analogues, qui fourniraient aux immigrés les contacts indispensables avec des autochtones et des employeurs tout en les familiarisant avec le fonctionnement du marché du travail, ces deux aspects étant décisifs pour accéder à des emplois hautement qualifiés. De telles mesures mériteraient d'être plus largement répandues, en coopération avec les employeurs. 
Le caractère fédéral du pays se reflète clairement dans la politique d'intégration, en fait, différentes mesures ont évolué aux niveaux local et cantonal pour promouvoir l'intégration. L'application du principe de subsidiarité a donné lieu à des mesures ponctuelles et flexibles visant de nombreux immigrés, mais en même temps a retardé le développement d'une politique fédérale d'intégration. En conséquence, et malgré une nette amélioration au cours de la dernière décennie, l'architecture générale en matière d'intégration demeure sous-développée. La politique fédérale d'intégration est somme toute modeste si on la compare aux actions menées dans d'autres pays de l'OCDE, dans la plupart desquels la population immigrée est moins importante qu'en Suisse. À l'exception de quelques instruments comme la formation linguistique élémentaire financée par l'Office fédéral des migrations, peu de mesures d'intégration ont été destinées en Suisse spécifiquement aux immigrés. En fait, l'approche globale de l'intégration repose sur l'idée que les immigrés vont bénéficier des services offerts à tous, plutôt que de concevoir des politiques ne visant que les immigrés. Afin de pallier les insuffisances du système actuel, la Confédération, les cantons et les autorités locales ont formulé récemment un certain nombre de suggestions pour améliorer le cadre général d'intégration, et se sont engagés à augmenter les ressources allouées à l'intégration.

Au niveau cantonal, les mesures d'aide à l'intégration varient beaucoup, ce qui tient en partie aux différences de taille et de composition des populations immigrées. Si de nombreux cantons ont intensifié leurs mesures d'intégration au cours des dernières années, ils l'ont souvent fait à petite échelle et dans le cadre de projets types, ce qui ne permet pas d'en évaluer facilement l'efficacité. Il faudrait que les autorités fédérales fixent quelques normes minimales, de sorte que tous les immigrés puissent bénéficier des mesures dont ils ont besoin, quel que soit leur canton de résidence.

L'accès à la nationalité suisse est difficile pour les immigrés : la durée de séjour requise est en effet particulièrement longue - 12 ans pour la procédure normale, soit le délai le plus long de l'OCDE - et le processus d'acquisition se déroule à trois niveaux, le candidat devant satisfaire aux exigences fédérales, cantonales et municipales. Une réforme de la législation est en cours, qui devrait remédier à certaines des lacunes les plus importantes de la loi sur la nationalité et améliorer la mobilité des migrants sur le territoire suisse. Les observations empiriques semblent indiquer que cela pourrait dynamiser fortement l'intégration des groupes d'immigrés défavorisés.

Dans l'ensemble, les performances des enfants d'immigrés sur le marché du travail sont bonnes en termes de comparaison internationale, ce qui est en partie dû aux bonnes conditions générales du marché du travail, et à d'autres facteurs tels que l'importance de l'apprentissage, qui semble particulièrement bénéfique pour les enfants d'immigrés dans le processus de transition de l'école vers le travail. Quelques programmes novateurs ont également été mis en place pour préparer les jeunes peu scolarisés (parmi lesquels une large part d'enfants d'immigrés) à l'apprentissage, et ils semblent avoir eu un effet bénéfique.

Les enfants d'immigrés dont les parents ont de faibles niveaux d'éducation obtiennent des résultats scolaires médiocres ; ils sont actuellement de plus en plus nombreux à entrer sur le marché du travail. Il semble que ces performances moins satisfaisantes puissent être attribuées, du moins en partie, à l'absence de prise en charge par le système éducatif à un stade suffisamment précoce, la scolarisation des tout jeunes enfants n'étant pas encore très développée en Suisse. Offrir aux enfants d'immigrés, dès l'âge critique de trois ou quatre ans, un enseignement à la fois adapté et ciblé, en même temps qu'un apprentissage de la langue, devrait s'imposer d'urgence comme une priorité de l'action publique.

Il est un domaine dans lequel la Suisse accuse un retard certain par rapport à d'autres pays de l'OCDE : celui de la lutte contre les discriminations. Les employeurs n'ont pas suffisamment conscience du problème, qui est par ailleurs absent du débat public. Des études ont pourtant montré qu'un enfant d'immigrés, en particulier si ses parents sont originaires de l'ex-Yougoslavie, doit présenter, à qualifications égales, jusqu'à cinq fois plus de candidatures qu'un enfant d'autochtones avant d'être convié à un entretien d'embauche. Il importerait de prendre les mesures requises pour atténuer ce type d'inégalité. 
Tout bien considéré, s'il ressort des comparaisons internationales que la Suisse obtient globalement de bons résultats quant à l'intégration de sa population immigrée sur le marché du travail, plusieurs signes révèlent néanmoins une situation divergente pour certaines catégories de migrants, quelques groupes désavantagés courant le risque d'être laissés sur le bord de la route. Il importe donc d'agir dès à présent, tant que les résultats d'ensemble restent positifs. La prise de conscience a eu lieu, et les efforts en faveur de l'intégration s'intensifient aux trois échelons du gouvernement. À de nombreux égards, cependant, les politiques d'intégration de la Suisse restent en deçà de celles menées par d'autres pays de l'OCDE. Un certain nombre de mesures devraient être envisagées pour remédier à cette situation, et pour faire en sorte que les résultats soient désormais satisfaisants pour toutes les catégories d'immigrés.

Mots clés : Intégration, immigrés, marché du travail, Suisse, compétences, reconnaissances des diplômes, discrimination

\section{Résumé des principales recommandations politiques}

\section{A) Renforcer le cadre global d'intégration}

- Développer des normes minimum communes pour que les mesures d'intégration s'appliquent dans tous les cantons.

- $\quad$ Faciliter l'échange de bonnes pratiques entre cantons et municipalités.

- S'assurer que tous les immigrés ayant besoin d'une aide à l'intégration y aient accès comme il convient, indépendamment du type de permis qu'ils possèdent et de la nature et de l'importance des prestations qu'ils perçoivent, notamment dans le cas des femmes immigrées.

- Offrir une formation linguistique à tous les immigrés qui en ont besoin, en tenant compte de leurs compétences et de leurs qualifications.

- Faciliter l'accession à la nationalité suisse, en réduisant en particulier les exigences des cantons et des communes en matière de durée de séjour pour faciliter la mobilité géographique des migrants.

- Sensibiliser aux avantages qu'implique l'acquisition de la nationalité suisse pour une meilleure intégration des immigrés et de leurs enfants.

\section{B) Favoriser une intégration rapide sur le marché du travail des migrants pour raisons humanitaires}

- Renforcer les incitations des cantons en faveur de l'intégration rapide sur le marché du travail des migrants pour raisons humanitaires au cours des cinq premières années de séjour.

- $\quad$ Mettre en œuvre un programme d'intégration structuré en faveur de tous les migrants pour raisons humanitaires arrivés récemment (requérants dont la demande est reconnue ou qui bénéficient de l'admission provisoire) en fonction des besoins des individus, en mettant clairement l'accent sur l'intégration sur le marché du travail.

- Mieux informer les employeurs au sujet de l'accès au marché du travail des personnes bénéficiant l'admission provisoire. 
Résumé des principales recommandations politiques (suite)

\section{C) Mieux utiliser les compétences des migrants}

- S'assurer que l'accent mis actuellement sur l'emploi peu qualifié des migrants pour raisons humanitaires n'empêche pas d'utiliser au mieux leurs compétences.

- Mieux faire connaître aux immigrés les offres disponibles en matière d'évaluation et de reconnaissance des qualifications acquises à l'étranger et mieux faire percevoir les avantages qu'implique cette reconnaissance.

- Mettre en place des formations passerelles et d'autres programmes de soutien pour aider les immigrés possédant des diplômes acquis à l'étranger à accéder à des emplois plus qualifiés.

- Envisager d'étendre avec discernement le bénéfice des subventions salariales temporaires aux immigrés.

D) Intensifier les efforts en faveur de l'intégration rapide des enfants d'immigrés

- Développer l'éducation préscolaire et accorder une attention particulière à la participation des enfants d'immigrés issus de milieux défavorisés dès l'âge de 3 ou 4 ans.

- Renforcer la formation linguistique des enfants d'immigrés, en particulier les plus jeunes.

- Examiner les causes des taux apparemment faibles d'achèvement de l'apprentissage par les enfants d'immigrés et prendre des mesures pour remédier à cette situation.

\section{E) Instaurer un cadre solide de lutte contre la discrimination}

- Rendre illégale la discrimination à l'embauche fondée sur la nationalité.

- Sensibiliser davantage les employeurs et la société en général à la question de la discrimination.

- $\quad$ Envisager de mettre en place des mesures plus volontaristes pour lutter contre la discrimination. 


\section{TABLE DES MATIÈRES}

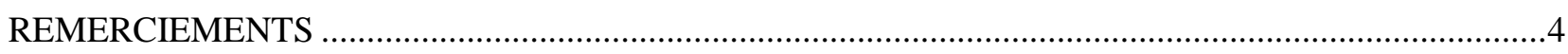

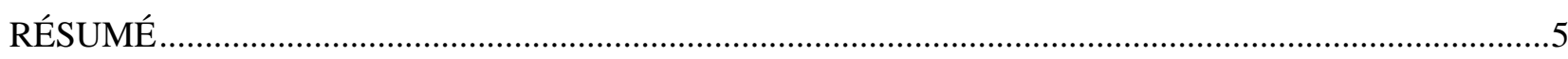

INTRODUCTION

I. PREMIER APERÇU DE LA SITUATION DES IMMIGRÉS ET DE LEURS ENFANTS SUR LE MARCHÉ DU TRAVAIL : COMPARAISON AVEC D'AUTRES PAYS ET ÉVOLUTION DANS LE TEMPS

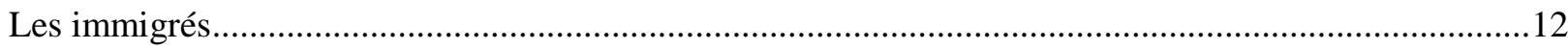

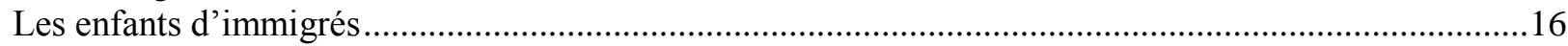

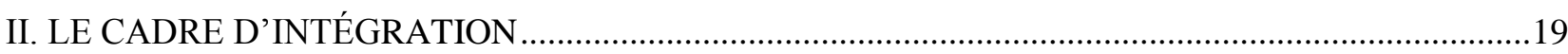

L'évolution des migrations à destination de la Suisse et les principaux groupes de migrants .................19

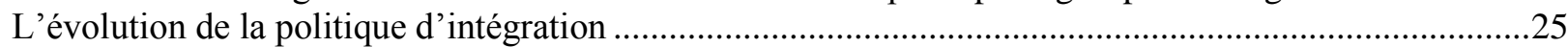

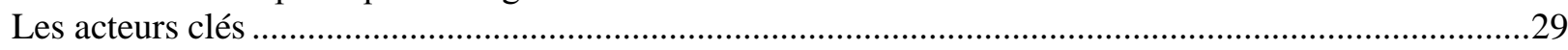

III. INTÉGRATION DES IMMIGRÉS SUR LE MARCHÉ DU TRAVAIL : PRINCIPALES

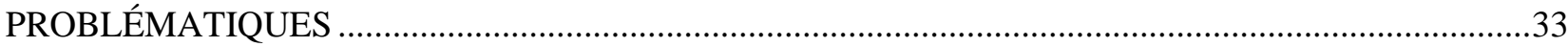

Qualifications et résultats en matière d'emploi.......................................................................................33

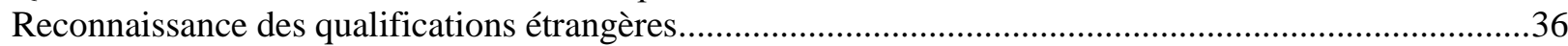

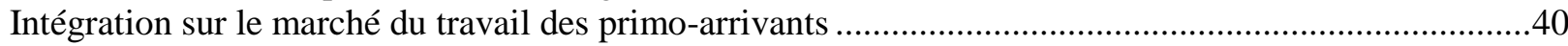

Participation des immigrés aux mesures actives du marché du travail ....................................................44

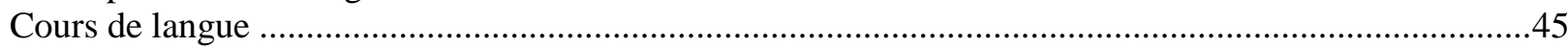

L'intégration sur le marché du travail des migrants pour raisons humanitaires ....................................46

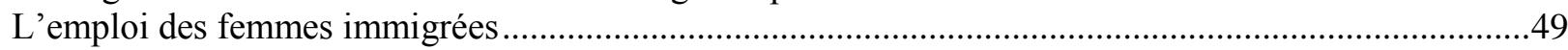

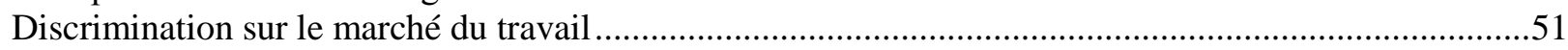

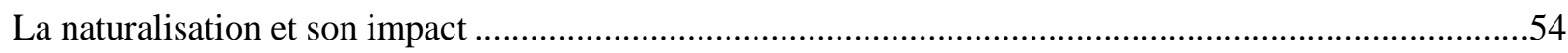

VI. L'INTÉGRATION DES ENFANTS D'IMMIGRÉS SUR LE MARCHÉ DU TRAVAIL ....................58

Résultats au regard de l'éducation des enfants d'immigrés en Suisse ......................................................58

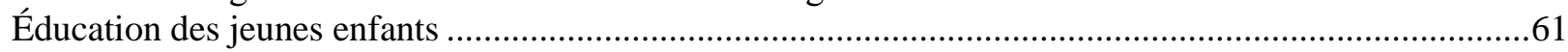

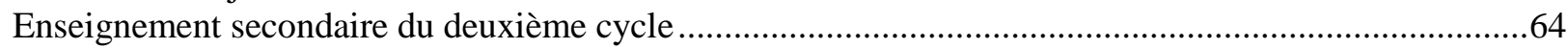

Processus de transition de l'école à l'emploi et résultats sur le marché du travail ...................................68

Intervention des pouvoirs publics pour faciliter la transition de l'école à l'emploi...................................71

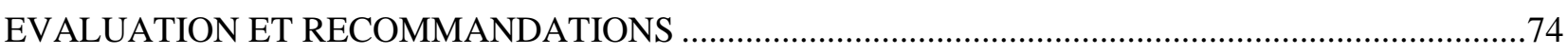

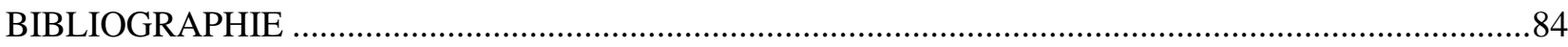

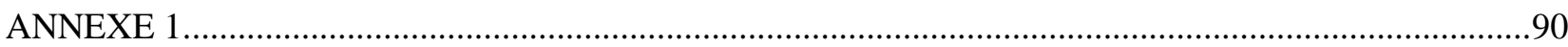

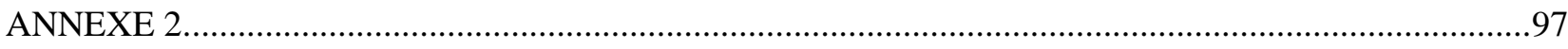

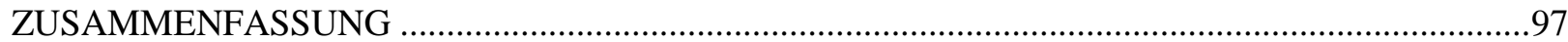

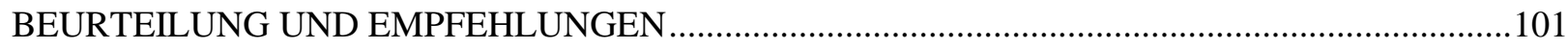

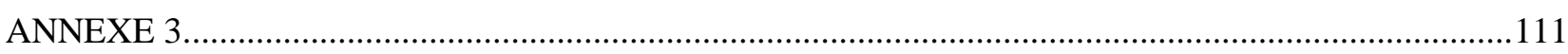

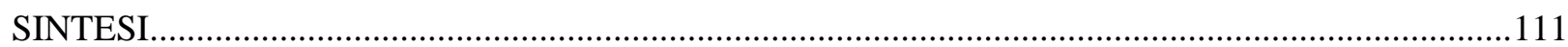

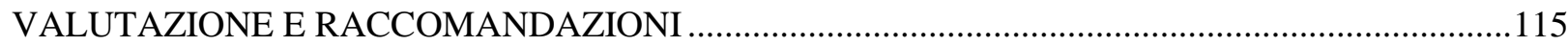


DELSA/ELSA/WD/SEM(2012)2

\section{L'INTÉGRATION DES IMMIGRÉS ET DE LEURS ENFANTS SUR LE MARCHÉ DU TRAVAIL EN SUISSE}

\section{Introduction}

1. Avec environ $27 \%$ de la population en âge de travailler née à l'étranger, la Suisse compte - avec l'Australie et le Luxembourg - l'une des plus fortes proportions d'immigrés, en termes relatifs, au sein de 1'OCDE. Cette situation tient au fait que la Suisse est depuis longtemps une terre d'immigration : en 1970, déjà, plus de $16 \%$ de sa population avaient une nationalité étrangère.

2. En Suisse, la population immigrée se caractérise, entre autres, par une forte concentration de ressortissants d'autres pays européens, en particulier des pays voisins - Italie, Allemagne et France -, ainsi que du Portugal. L'immense majorité venait initialement d'Italie - c'était le cas, en 1960, de $63 \%$ des étrangers présents sur le territoire. Avec le temps, les flux se sont diversifiés, englobant des immigrés venus d'autres pays du sud de l'Europe, en particulier de l'ex-Yougoslavie. ${ }^{1}$ Plus récemment, suite à l'instauration progressive par la Suisse de la libre circulation avec les pays membres de l'Union européenne, la Suisse a enregistré un afflux important d'immigrés venant d'Allemagne. Ce sont aujourd'hui quasiment $60 \%$ des immigrés qui sont issus de l'Espace économique européen (EEE), $17 \%$ venant de l'ex-Yougoslavie.

3. Les migrations vers la Suisse sont essentiellement des migrations de travail, avec les flux connexes liés au regroupement familial. Il s'ensuit que l'intégration des immigrés sur le marché du travail a moins été un enjeu, dans le débat public, que la question de l'impact macroéconomique de l'immigration sur les salaires et les changements structurels.

4. Au fil des vingt dernières années, suite à la chute du Rideau de fer, la Suisse est également devenue l'un des principaux pays de destination pour les migrations humanitaires, ce qui a accentué la diversification des flux. Parallèlement à cette diversification et à l'arrivée de migrants de pays plus lointains pour des raisons autres que le travail, la question de l'intégration sur le marché du travail a gagné en importance dans le débat public, sans atteindre, toutefois, l'acuité qu'elle revêt dans bon nombre d'autres pays de l'OCDE où le nombre d'immigrés est pourtant nettement plus faible.

5. Il est indispensable de s'attarder aussi, pour analyser cet aspect, sur trois grandes particularités structurelles de la Suisse. La première réside dans la décentralisation des décisions politiques, auxquelles contribuent activement les trois échelons administratifs (fédéral, cantonal et local), et dans l'hétérogénéité linguistique du pays, où coexistent quatre langues officielles (allemand, français, italien, romanche). La deuxième particularité tient à la souplesse du marché du travail, qui se distingue par un fort taux d'activité chez les hommes et les femmes, un nombre limité de chômeurs, des rémunérations élevées et des taux d'indemnisation relativement faibles en cas de chômage par rapport à d'autres pays européens de l'OCDE. Enfin, dotée d'une économie très productive et fortement internationalisée, la Suisse enregistre un PIB par habitant parmi les plus élevés de l'OCDE. L'économie suisse repose à plus de $40 \%$ sur les exportations.

6. Le présent rapport s'articule comme suit: la section I présente, sous forme de comparaison internationale, un tableau général des résultats enregistrés sur le marché du travail par les immigrés en Suisse, et s'intéresse également à l'évolution de ces chiffres dans le temps. La section II expose le cadre de

Pour plus de facilité, les termes "ex-Yougoslavie" et "ancienne Yougoslavie" sont employés indifféremment, dans le présent document, pour désigner l'ancienne République fédérative de Yougoslavie et les pays qui en sont issus. La Slovénie, qui fait partie de ces pays mais est aujourd'hui membre de l'Union européenne et de l'OCDE, n'est généralement pas prise en compte dans ces chiffres. 
l'intégration, c'est-à-dire l'évolution et la composition actuelle de la population immigrée, les grands volets de la politique d'intégration, et les acteurs clés de l'intégration des migrants sur le marché du travail. La section III met l'accent sur les principales problématiques de l'intégration. Enfin, la section IV s'intéresse à l'intégration des enfants d'immigrés en termes d'emploi. Le rapport s'achève par une synthèse et par des recommandations. 


\section{PREMIER APERÇU DE LA SITUATION DES IMMIGRÉS ET DE LEURS ENFANTS SUR LE MARCHÉ DU TRAVAIL : COMPARAISON AVEC D'AUTRES PAYS ET ÉVOLUTION DANS LE TEMPS}

\section{Les immigrés}

7. Une comparaison internationale de la situation des migrants sur le marché du travail montre en premier lieu, de façon très nette, que les taux d'emploi les plus élevés, pour les hommes comme pour les femmes, se trouvent en Suisse (tableau 1). ${ }^{2}$ Le tableau est moins satisfaisant en matière de chômage : les taux sont, en effet, de deux à trois fois supérieurs chez les immigrés par rapport aux autochtones. Il importe cependant de prendre en compte le taux de chômage global relevé en Suisse, le plus faible du groupe témoin après celui de la Norvège.

Tableau 1 : Caractéristiques de l'emploi des personnes nées dans le pays et des personnes nées à l'étranger, 15-64 ans, dans une sélection de pays de l'OCDE, moyenne 2008/2009

\begin{tabular}{|c|c|c|c|c|c|c|c|c|c|c|c|c|c|}
\hline & \multirow{2}{*}{\multicolumn{2}{|c|}{\begin{tabular}{cc|} 
& $\%$ de \\
$\%$ de la & personnes nées \\
dans un pays à \\
population & bas revenu \\
née à & parmi les \\
l'étranger & personnes nées \\
& à l'étranger
\end{tabular}}} & \multicolumn{3}{|c|}{ Taux de participation } & \multicolumn{4}{|c|}{ Taux d'emploi } & \multicolumn{4}{|c|}{ Taux de chômage } \\
\hline & & & $\begin{array}{c}\text { Personnes } \\
\text { nées dans } \\
\text { le pays }\end{array}$ & $\begin{array}{c}\text { Personnes } \\
\text { nées à } \\
\text { l'étranger }\end{array}$ & $\begin{array}{l}\text { Personnes } \\
\text { nées dans un } \\
\text { pays à bas } \\
\text { revenu }\end{array}$ & $\begin{array}{l}\text { Personnes } \\
\text { nées dans } \\
\text { le pays }\end{array}$ & $\begin{array}{c}\text { Personnes } \\
\text { nées à } \\
\text { l'étranger }\end{array}$ & $\begin{array}{l}\text { Personnes } \\
\text { nées dans un } \\
\text { pays à bas } \\
\text { revenu }\end{array}$ & $\begin{array}{c}\text { Différence nés } \\
\text { dans le pays- } \\
\text { nés à } \\
\text { l'étranger } \\
\text { (points de } \\
\text { pourcentage) }\end{array}$ & $\begin{array}{l}\text { Personnes } \\
\text { nées dans } \\
\text { le pays }\end{array}$ & $\begin{array}{c}\text { Personnes } \\
\text { nées à } \\
\text { l'étranger }\end{array}$ & $\begin{array}{c}\text { Personnes } \\
\text { nées dans } \\
\text { un pays à } \\
\text { bas revenu }\end{array}$ & $\begin{array}{c}\text { Différence nés } \\
\text { dans le pays- } \\
\text { nés à } \\
\text { l'étranger } \\
\text { (points de } \\
\text { pourcentage) }\end{array}$ \\
\hline \multicolumn{14}{|l|}{ Hommes } \\
\hline Autriche & 16.4 & 49 & 81.2 & 81.0 & 81.0 & 78.5 & 73.7 & 71.5 & 4.8 & 3.4 & 9.0 & 11.6 & 5.6 \\
\hline Australie (1) & 28.1 & $\ldots$ & 83.8 & 80.5 & $\ldots$ & 79.7 & 76.1 & & 3.6 & 4.9 & 5.5 & & 0.6 \\
\hline Belgique & 12.3 & 51 & 73.0 & 74.0 & 74.1 & 68.6 & 62.2 & 57.8 & 6.4 & 5.9 & 15.9 & 22.0 & 10.0 \\
\hline Canada (2) & 20.3 & $\ldots$ & 82.0 & 83.2 & $\ldots$ & 75.5 & 75.8 & & -0.3 & 7.9 & 8.8 & & 0.9 \\
\hline Danemark & 8.4 & 49 & 84.6 & 80.2 & 77.6 & 80.7 & 73.4 & 70.2 & 7.4 & 4.5 & 8.5 & 9.5 & 4.0 \\
\hline France & 11.9 & 66 & 75.1 & 77.2 & 76.4 & 69.6 & 66.9 & 64.3 & 2.7 & 7.4 & 13.4 & 15.9 & 5.9 \\
\hline Allemagne & 15.1 & & 82.2 & 82.6 & & 76.4 & 72.0 & & 4.4 & 7.0 & 12.8 & & 5.8 \\
\hline Pays-Bas & 12.2 & 76 & 85.8 & 79.9 & 79.1 & 83.4 & 73.9 & 72.4 & 9.5 & 2.8 & 7.6 & 8.5 & 4.8 \\
\hline Norvège & 9.0 & 53 & 82.0 & 81.3 & 75.5 & 79.8 & 74.4 & 66.5 & 5.4 & 2.7 & 8.5 & 12.0 & 5.8 \\
\hline Suède & 15.2 & 59 & 81.9 & 79.3 & 78.2 & 76.7 & 68.3 & 63.6 & 8.5 & 6.3 & 14.0 & 18.6 & 7.6 \\
\hline Suisse & 27.2 & 33 & 87.6 & 88.9 & 87.8 & 85.4 & 83.9 & 80.1 & 1.5 & 2.5 & 5.6 & 8.8 & 3.1 \\
\hline $\begin{array}{l}\text { Suisse alémanique (3) } \\
\text { Suisse romande ou }\end{array}$ & 24.8 & 49 & 88.9 & 89.3 & 86.9 & 87.1 & 84.7 & 80.9 & 2.4 & 2.0 & 5.2 & 7.1 & 3.1 \\
\hline italienne (3) & 33.5 & 33 & 84.0 & 88.1 & 86.0 & 80.5 & 82.4 & 77.4 & -1.9 & 4.3 & 6.6 & 10.2 & 2.3 \\
\hline Royaume-Uni & 13.7 & 63 & 81.9 & 83.2 & 80.9 & 75.8 & 76.9 & 73.8 & $\begin{array}{l}-1.1 \\
\end{array}$ & 7.5 & 7.6 & 8.9 & 0.1 \\
\hline États-Unis & 16.7 & 89 & 77.4 & 86.0 & 86.3 & 70.5 & 79.1 & 79.0 & -8.6 & 8.6 & 8.0 & 8.5 & $(-0.6)$ \\
\hline Moyenne (4) & 15.9 & 59 & 81.4 & 81.3 & 79.7 & 77.0 & 73.6 & 69.9 & 3.4 & 5.5 & 9.6 & 12.4 & 4.1 \\
\hline \multicolumn{14}{|l|}{ Femmes } \\
\hline Autriche & 18.1 & 43 & 70.7 & 62.0 & 55.5 & 68.1 & 57.0 & 49.7 & 11.1 & 3.7 & 8.0 & 10.3 & 4.3 \\
\hline Australie (1) & 28.4 & $\ldots$ & 72.6 & 64.0 & $\ldots$ & 69.1 & 60.1 & $\ldots$ & 9.0 & 4.9 & 6.0 & $\ldots$ & 1.1 \\
\hline Belgique & 13.2 & 50 & 62.3 & 51.4 & 46.4 & 58.0 & 43.2 & 36.2 & 14.8 & 6.9 & 16.0 & 22.0 & 9.1 \\
\hline Canada (2) & 21.6 & $\ldots$ & 75.6 & 69.7 & $\ldots$ & 71.2 & 63.7 & $\ldots$ & 7.5 & 5.8 & 8.6 & $\ldots$ & 2.8 \\
\hline Danemark & 9.7 & 51 & 78.3 & 66.8 & 60.7 & 75.1 & 60.9 & 54.5 & 14.2 & 4.2 & 8.9 & 10.3 & 4.8 \\
\hline France & 12.5 & 65 & 67.3 & 59.7 & 55.4 & 61.6 & 51.4 & 46.4 & 10.2 & 8.5 & 13.8 & 16.2 & 5.4 \\
\hline Allemagne & 16.0 & $\ldots$ & 72.9 & 62.3 & $\ldots$ & 68.0 & 54.5 & & 13.5 & 6.6 & 12.5 & $\ldots$ & 5.9 \\
\hline Pays-Bas & 13.6 & 73 & 75.1 & 61.6 & 57.5 & 72.8 & 57.2 & 52.9 & 15.5 & 3.1 & 7.0 & 8.0 & 4.0 \\
\hline Norvège & 9.7 & 59 & 77.1 & 72.3 & 68.1 & 75.4 & 68.3 & 63.4 & 7.1 & 2.2 & 5.5 & 7.0 & 3.3 \\
\hline Suède & 17.4 & 57 & 78.6 & 67.6 & 63.7 & 73.7 & 58.3 & 52.0 & 15.4 & 6.2 & 13.7 & 18.4 & 7.5 \\
\hline Suisse & 28.0 & 36 & 78.4 & 73.3 & 68.2 & 76.0 & 67.6 & 59.5 & 8.3 & 3.1 & 7.7 & 12.8 & 4.7 \\
\hline $\begin{array}{l}\text { Suisse alémanique (3) } \\
\text { Suisse romande ou }\end{array}$ & 25.5 & 51 & 80.0 & 74.2 & 69.1 & 77.9 & 68.8 & 61.5 & 9.0 & 2.8 & 7.4 & 11.2 & 4.6 \\
\hline $\begin{array}{l}\text { Suisse romande ou } \\
\text { italienne (3) }\end{array}$ & 34.7 & 39 & 74.1 & 71.9 & 68.7 & 71.0 & 65.9 & 60.1 & 5.1 & 4.3 & 8.5 & 12.7 & 4.2 \\
\hline Royaume-Uni & 14.2 & 62 & 70.4 & 62.9 & 55.9 & 66.5 & 58.2 & 50.6 & 8.3 & 5.6 & 7.5 & 9.5 & 2.0 \\
\hline États-Unis & 15.7 & 88 & 68.9 & 62.9 & 62.3 & 64.7 & 58.4 & 57.7 & 6.3 & 6.1 & 7.1 & 7.4 & 1.0 \\
\hline Moyenne (4) & 16.8 & 59 & 72.9 & 64.3 & 59.4 & 69.2 & 58.4 & 52.3 & 10.9 & 5.1 & 9.4 & 12.2 & 4.3 \\
\hline
\end{tabular}

Notes : 1. Les données se réfèrent à la moyenne janvier 2008 - juin 2009. 2. Pour le Canada, des données distinctes pour les hommes et les femmes n'étaient pas disponibles pour les personnes nées dans un pays à bas revenu. 3 . Données de l'Enquête suisse sur la population active (ESPA Office fédéral de la statistique suisse) 2008/2009. 4. la moyenne se réfère à la moyenne non pondérée de tous les pays compris dans le tableau. La définition des pays à bas revenu diffère légèrement entre ESPA et l'Enquête européenne sur la population active. Les "pays à bas revenu" se réfèrent à tous les pays autres que les pays de l'OCDE à haut revenu.

Source : Enquête européenne sur la population active, sauf la Suisse par région (ESPA), les États-Unis (Current Population Survey March Supplement), le Canada (Enquête sur la population active) et l'Australie (Enquête sur la population active). témoin comprend des pays de l'OCDE ayant aussi, depuis longtemps, de fortes populations immigrées, dont beaucoup ont déjà fait l'objet d'une étude de l'OCDE. 
8. Ces bons résultats sont, dans une large mesure, liés au fait que la majorité des immigrés approximativement les deux tiers, soit la plus forte proportion au sein du groupe de pays soumis à l'étude comparative - proviennent de pays de l'OCDE à haut revenu. ${ }^{3}$ Dans la plupart des pays, l'intégration sur le marché du travail est généralement plus facile pour cette catégorie de migrants (voir également OCDE, 2007, 2008b). Différents facteurs expliquent ce phénomène, par exemple le fait que de nombreuses personnes originaires de pays à faible revenu sont des migrants humanitaires. En outre, au moment de la migration, le marché du travail et le système éducatif dans ces pays étaient souvent très différents de ceux des pays de l'OCDE à revenu élevé. ${ }^{4}$

9. Si l'on considère uniquement les immigrés issus de pays à faible revenu, on constate que la situation reste relativement favorable pour les hommes, alors que la situation des femmes issues de ces pays vis-à-vis du marché du travail est globalement similaire à celle observée dans d'autres pays de l'OCDE, comme le Danemark et la Norvège, qui bénéficient d'un marché du travail dynamique.

10. Le tableau 1 montre aussi que les différences sont ténues entre la partie germanophone, d'une part, et les parties francophone et italophone de la Suisse. On relève cependant un taux d'emploi plus élevé et un niveau de chômage plus faible du côté germanophone. Cela se vérifie également pour les immigrés, mais dans une moindre mesure. De plus, la proportion de migrants originaires de pays à faible revenu principalement l'ancienne Yougoslavie et la Turquie - est supérieure dans les cantons de langue allemande.

11. Les données relatives à la situation des migrants en matière d'emploi sont fournies exclusivement par l'Enquête suisse sur la population active (ESPA), qui n'enregistre régulièrement que depuis 2003 les informations concernant le pays de naissance : il est donc difficile d'analyser comment l'intégration des immigrés et de leurs enfants sur le marché du travail évolue au fil du temps. ${ }^{5}$ De fait, le terme «immigrés » évoque généralement pour les Suisses, dans le débat public comme dans les données et travaux administratifs, les personnes de nationalité étrangère (voir encadré 1).

Encadré 1. Intégration des immigrés et de leurs enfants sur le marché du travail en Suisse : population cible, données et recherches

En dépit du nombre important d'immigrés installés en Suisse et de l'attention croissante que les pouvoirs publics accordent à leur intégration sur le marché du travail, peu d'analyses quantitatives ont été consacrées à cette question. L'une des raisons vient de ce que les textes juridiques (comme l'Ordonnance sur l'intégration des étrangers,

OIE) et les bases de données administratives fournissant des informations sur l'intégration des «immigrés » en termes d'emploi visent non pas les populations nées à l'étranger, mais les ressortissants étrangers. La prépondérance du critère de nationalité gêne les comparaisons internationales, car les lois sur la nationalité et le nombre de naturalisations varient considérablement entre les différents pays de l'OCDE.

Sauf indication contraire, l'expression «pays à revenu élevé » désigne les pays de l'OCDE ayant un revenu important (pays de l'OCDE, sauf le Mexique et la Turquie). Les « pays à faible revenu » sont tous les autres pays d'origine.

4

Il convient de souligner que la distinction entre pays à faible revenu/à revenu élevé dissimule une certaine hétérogénéité au sein même de ces groupes. Ainsi, comme on le verra plus loin, les migrants originaires du Portugal - pays à revenu élevé sont souvent confrontés à d'importantes difficultés en matière d'emploi. Cela s'explique en partie par un faible niveau d'études et conduit également à une situation peu satisfaisante pour leurs enfants (voir aussi Fibbi et al., 2010).

On dispose aussi d'informations sur le pays de provenance pour les années 1998 et 2001. Jusqu'en 2001, toutefois, seuls 16000 ménages étaient couverts par l'Enquête suisse sur la population active, dont les résultats relatifs à la population immigrée ne sont pas fiables. En 2001, l'échantillon a été porté à 35000 ménages. Depuis 2003, l'enquête comprend systématiquement une question sur le pays de provenance. En outre, et depuis 2003 également, 15000 ménages supplémentaires ayant un étranger pour chef de famille sont sélectionnés à partir du Système d'information central sur les migrations (le SYMIC). Depuis 2010, l'Enquête sur la population active s'effectue de manière continue, et l'échantillon a beaucoup été élargi (105 000 ménages, auxquels s'ajoutent 21000 ménages étrangers supplémentaires). 


\section{Encadré 1. Intégration des immigrés et de leurs enfants sur le marché du travail en Suisse : population cible, données et recherches (suite)}

Si la nationalité australienne, belge ou canadienne peut être obtenue après seulement trois ans dans le pays, la Suisse applique des règles relativement strictes dans ce domaine et, en particulier, n'accorde pas automatiquement la nationalité à une personne née en Suisse de parents immigrés (voir section III ci-après). De plus, il semble que la naturalisation soit sélective : les personnes qui acquièrent la nationalité suisse bénéficient souvent d'une éducation plus poussée et trouvent plus facilement leur place sur le marché du travail. Cela peut entraîner une aggravation des résultats concernant les "étrangers ", même si la situation réelle des migrants reste globalement constante, voire s'améliore. Par ailleurs, la Suisse étant depuis longtemps une terre d'immigration et l'accès à la nationalité y étant difficile, plus d'un étranger sur cinq est un enfant d'immigré né en Suisse. Malgré tout, les enjeux ne sont pas les mêmes pour les personnes nées et éduquées dans le pays hôte, et pour celles qui ont immigré, le plus souvent alors qu'elles étaient déjà adultes, et qui ont acquis à l'étranger au moins une partie de leur capital humain. Pour toutes ces raisons, le présent document s'intéresse, autant que possible, aux personnes nées à l'étranger plutôt qu'à celles qui ont une nationalité étrangère. Les enfants nés en Suisse de parents immigrés étant nombreux, une section distincte est consacrée à la question de leur intégration.

Un autre facteur vient compliquer les comparaisons internationales relatives à l'intégration des immigrés sur le marché du travail : en effet, la plupart de ces personnes sont originaires de l'Espace économique européen (Union européenne élargie, Norvège, Islande et Liechtenstein). Non seulement les migrants issus de ces pays parviennent mieux à trouver un emploi dans la plupart des pays de destination, mais ils peuvent facilement retourner dans leur pays d'origine en cas de chômage, et bénéficient en Suisse d'une série de dispositions favorables quant à la mobilité professionnelle et la reconnaissance de leurs qualifications. De façon générale, la Suisse considère que le problème de l'intégration sur le marché du travail se pose essentiellement pour les migrants venus de pays extérieurs à l'Espace économique européen (EEE). Le présent rapport cible donc surtout les immigrés originaires de pays non EEE, ainsi que leurs enfants.

Les données concernant les personnes nées à l'étranger ont longtemps été peu abondantes, mais la situation s'est sensiblement améliorée ces dernières années. L'Enquête suisse sur la population active comporte régulièrement des modules consacrés à l'immigration : ce fut le cas en 1998, 2001, 2003 et 2008. Le plus récent de ces modules fournit également de nombreuses indications sur la participation aux services d'intégration et sur la reconnaissance de qualifications acquises à l'étranger; il permet aussi le recensement des enfants d'immigrés nés en Suisse. Depuis 2003, l'enquête procède à un suréchantillonnage des étrangers, et mentionne le pays de naissance des sondés. Depuis 2009, les informations issues de cette enquête peuvent en outre être reliées aux données administratives des caisses d'assurances sociales; on dispose ainsi d'éléments remontant à 1999. Toutefois, on ne dispose que d'informations très globales sur les types de mesures d'intégration auxquelles un individu prend part.

La Suisse a également participé à l'Enquête de 2003 sur la littératie et les compétences des adultes (ALL). L'échantillon suisse se composait de 1087 immigrés et de 334 personnes nées en Suisse de deux parents immigrés, ce qui a permis quelques analyses de base dans le cadre de cette étude (voir plus loin).

Le principal ensemble de données longitudinales est le Panel suisse de ménages (PSM). Lancé en 1999 avec 5074 ménages participants, il contient des informations sur un large éventail de questions liées à l'intégration, et notamment sur les parents des personnes interrogées. Cependant, la modestie de l'échantillon et le phénomène d'attrition limitent les possibilités d'exploitation de cette source de données en vue d'une analyse longitudinale de l'intégration des immigrés sur le marché du travail. L'intégration du PSM dans l'Enquête de l'Union européenne sur le revenu et les conditions de vie (EU-SILC) permet néanmoins de faire bouger progressivement les choses. En 2011, un module spécial couvre la transmission intergénérationnelle des handicaps sociaux.

La base de données TREE (Transition de l'école à l'emploi) facilite l'analyse longitudinale de l'intégration des enfants d'immigrés sur le marché du travail. Elle porte sur 5528 personnes ayant participé à l'enquête PISA de 2000 en Suisse, dont elle a suivi le passage de l'école à l'emploi en huit tranches annuelles jusqu'en 2007, une neuvième tranche ayant été examinée en 2010. La base de données contient des informations sur les performances scolaires et sur les antécédents d'immigration des sondés, ce qui permet une étude approfondie de l'intégration des enfants d'immigrés dans sa dimension longitudinale. Cet ensemble de données a été utilisé pour l'analyse, présentée ci-après, relative aux enfants d'immigrés. 


\section{Encadré 1. Intégration des immigrés et de leurs enfants sur le marché du travail en Suisse : population} cible, données et recherches (suite)

Différentes institutions et initiatives ont récemment donné une impulsion aux recherches sur l'intégration des immigrés ; le volet « marché du travail » n'est cependant pas toujours le premier sujet d'intérêt. En 1995, le Forum suisse pour l'étude des migrations et de la population a été créé à l'Université de Neuchâtel. Ce Forum effectue des recherches, dans une perspective interdisciplinaire, sur les questions de migration et d'intégration. Entre 2003 et 2008 , le Fonds national suisse a financé un programme de recherche ambitieux consacré à l'intégration, pour un montant total de 13 millions CHF. Il s'agissait du deuxième grand programme-cadre sur les migrations et l'intégration ; en effet, le Fonds avait déjà financé, pour un montant de 8 millions CHF, des travaux de recherche relatifs aux migrations et aux relations interculturelles qui avaient pris fin en 2000 .

12. Le graphique 1 retrace l'évolution, depuis 2003, du taux d'emploi chez les immigrés en Suisse. S'agissant des hommes, on observe une amélioration régulière depuis 2003, à la fois en termes absolus et par rapport aux autochtones. Cela peut s'expliquer, dans une certaine mesure, par le fait que la population immigrée comprend davantage de ressortissants de pays à revenu élevé. Il est, de fait, surprenant de constater que le taux d'emploi de cette catégorie de population est en corrélation quasi-parfaite avec celui des autochtones pour l'ensemble de la période considérée. En ce qui concerne les personnes nées dans des pays à faible revenu, la situation s'est sensiblement améliorée dans le domaine de l'emploi, les écarts ayant presque diminué de moitié depuis 2003.

13. Chez les femmes, on constate la même corrélation à peu près parfaite entre le taux d'emploi des immigrées issues de pays OCDE à revenu élevé et celui des autochtones. Il existe néanmoins des décalages importants pour les femmes originaires de pays à faible revenu. Par ailleurs, et contrairement à ce que l'on relève chez les hommes, l'écart se creuse à nouveau entre le niveau d'intégration des femmes autochtones et celui des immigrées issues de pays à faible revenu sur le marché du travail. Les chiffres n'en restent pas moins assez élevés par comparaison avec d'autres pays (voir tableau 1), le taux d'emploi se situant aux environs de $60 \%$.

\section{Graphique 1 : Évolution du taux d'emploi depuis 2003, par pays de naissance, 15-64 ans Hommes Femmes}
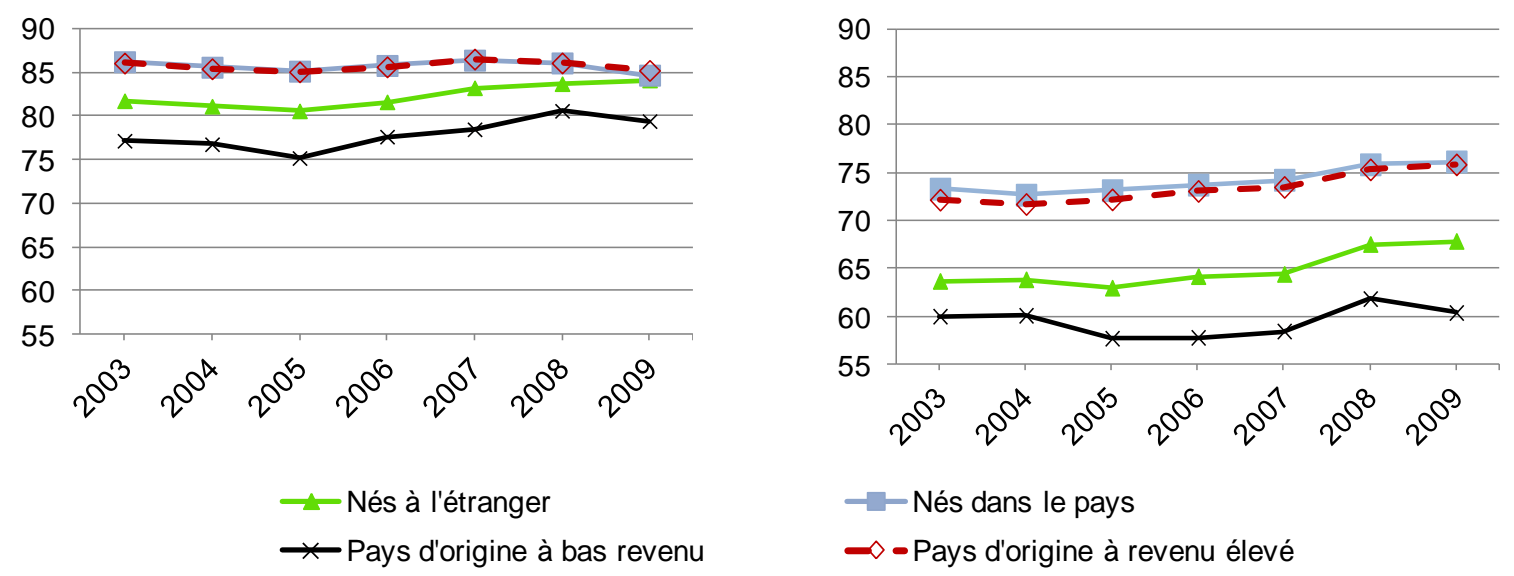

Source : Enquête suisse sur la population active, Office fédéral de la statistique 2003-2009. 


\section{Graphique 2 : Évolution du taux de chômage depuis 2003, par pays de naissance, 15-64 ans}
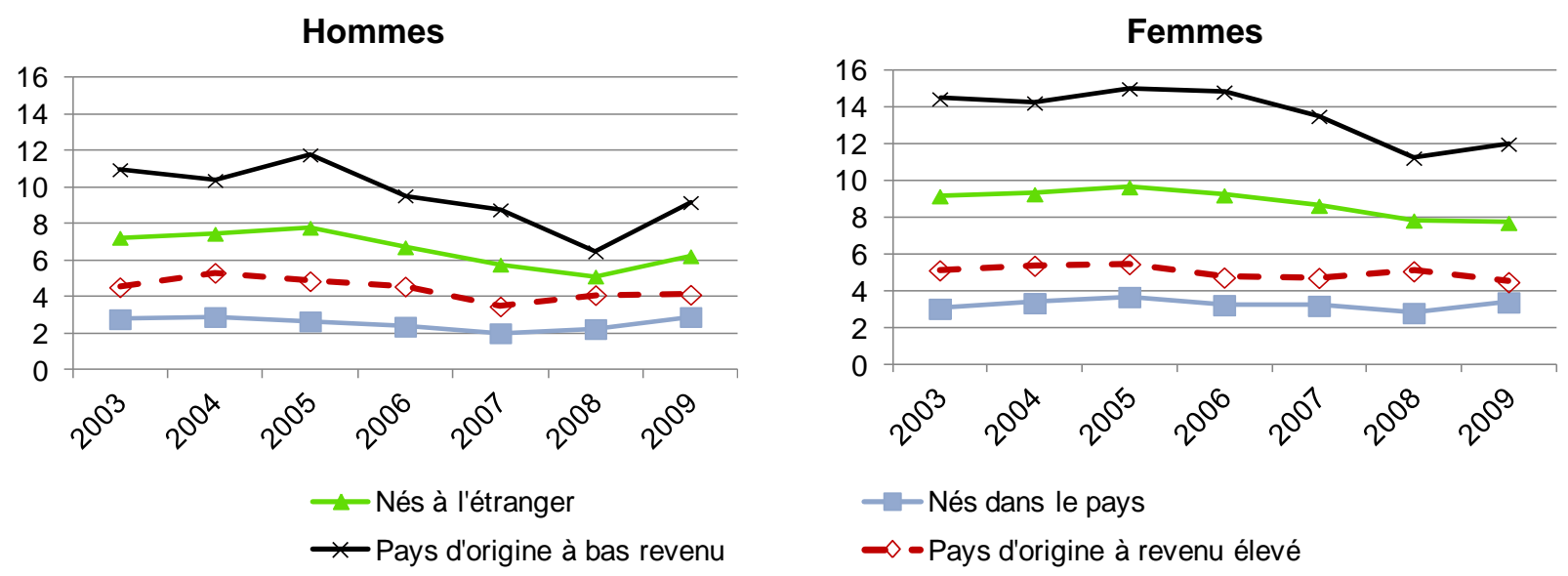

Source : Enquête suisse sur la population active, Office fédéral de la statistique 2003-2009.

14. Le graphique 2 illustre l'évolution de la situation dans le domaine du chômage. On constate un taux de chômage plus élevé pour tous les groupes d'immigrés, ainsi que des écarts particulièrement prononcés, là encore, chez les femmes originaires de pays à faible revenu. Cependant, le taux de chômage des immigrés issus de pays à faible revenu - hommes et femmes - a enregistré une baisse non négligeable entre 2005 et 2008, diminuant même quasiment de moitié par rapport à 2005 dans le cas des hommes. Le taux de chômage de cette catégorie de population a toutefois connu une envolée démesurée avec la crise.

15. Un examen rapide des rémunérations des immigrés fait apparaître que, lorsqu'ils ont un emploi, ils sont très nombreux à figurer parmi les bas salaires (voir Annexe, graphique 1). Une fois de plus, les disparités s'avèrent assez fortes entre les immigrés issus de pays à revenu élevé et ceux qui proviennent de pays à faible revenu, ces derniers étant nettement moins bien lotis. Vingt-huit pour cent de cette deuxième catégorie d'immigrés gagnent moins des deux tiers du salaire horaire médian pour un emploi à temps plein, contre $17 \%$ des personnes originaires de pays à revenu élevé et $20 \%$ des autochtones. Dans le même temps, les ressortissants de pays à faible revenu sont sous-représentés dans les hauts salaires (plus de $166 \%$ du salaire horaire médian pour un emploi à temps plein).

\section{Les enfants d'immigrés}

16. A leur arrivée dans le pays hôte, les migrants se heurtent souvent à de grosses difficultés pour entrer sur le marché du travail, en raison de leur capital humain différent de celui des autochtones. Les enfants nés en Suisse de parents immigrés, en revanche, ne devraient pas être confrontés aux mêmes problèmes puisqu'ils ont été formés dans la langue et dans le système éducatif du pays d'accueil. Ils devraient donc obtenir a priori, au regard de l'emploi, sensiblement les mêmes résultats que les enfants d'autochtones, et ils sont souvent considérés comme le marqueur en matière d'intégration (Card, 2004).

17. On dispose de données internationalement comparables concernant l'intégration des enfants d'immigrés sur le marché du travail pour la tranche d'âge 20-29 ans. Dans la quasi-totalité des pays de l'OCDE utilisés pour la comparaison, les enfants nés en Suisse de parents immigrés affichent un taux d'emploi nettement inférieur à celui des enfants d'autochtones (voir graphique 3). En Suisse, par contre, l'écart se révèle pour ainsi dire négligeable. S'agissant des femmes, le taux d'emploi des enfants nés sur le territoire suisse de parents immigrés peut même dépasser celui des enfants d'autochtones. Les enfants d'immigrés - hommes et femmes - connaissent dans ce pays le taux d'emploi le plus élevé de la zone OCDE. 


\section{Graphique 3 : Taux d'emploi des enfants de personnes nées dans le pays et des enfants d'immigrés nés dans le pays, par genre, 20-29 ans et non scolarisés, autour de 2008}
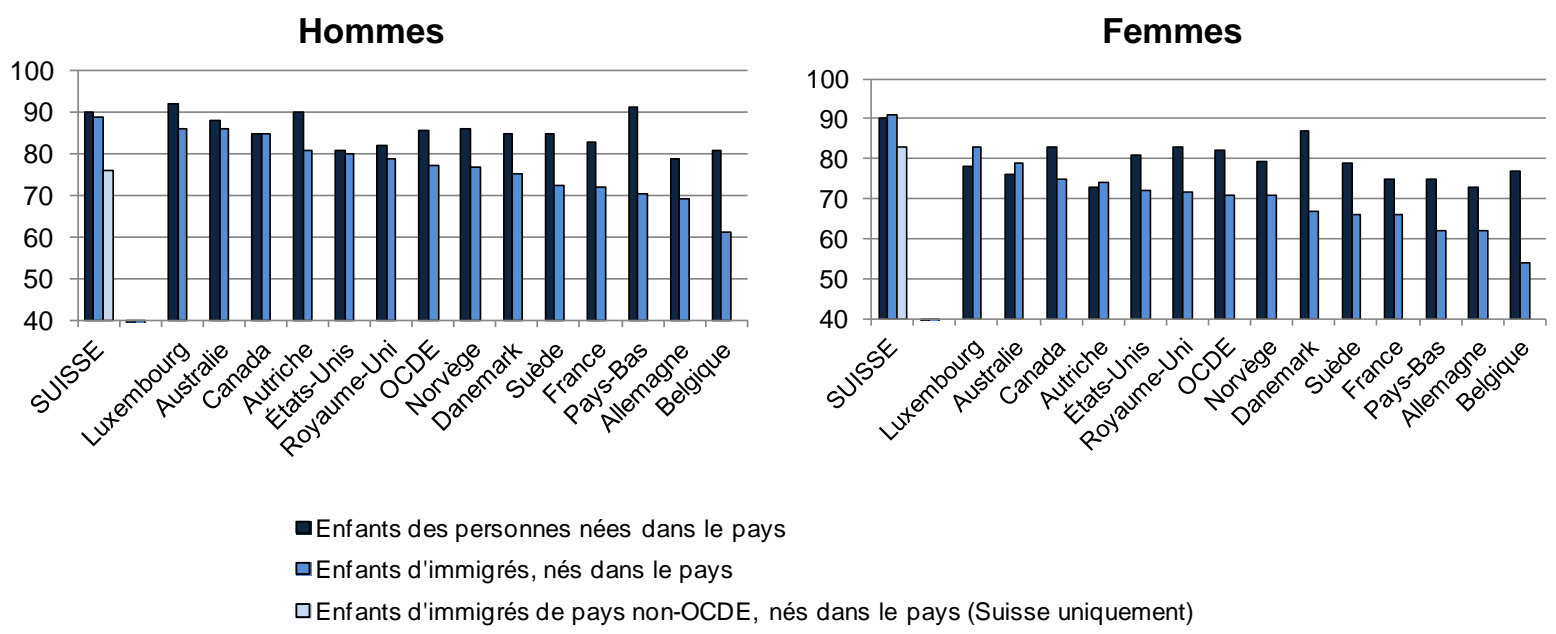

Source : Pour la Suisse, Enquête suisse sur la population active, (Office fédéral de la statistique). Pour les autres pays et pour les notes, cf. Liebig et Widmaier (2010).

18. Ce tableau encourageant vaut aussi pour un groupe de population qui préoccupe spécialement les pouvoirs publics : les jeunes peu qualifiés non scolarisés, qui ne suivent aucune formation et n'occupent aucun emploi («neither in employment nor in education or training » - NEET). C'est encore en Suisse, par comparaison avec les autres pays de l'OCDE, que cette catégorie de population à risque est le plus faiblement représentée parmi les enfants nés sur le territoire de parents immigrés (graphique 4). ${ }^{6}$

\section{Graphique 4 : "Population à risque" parmi les enfants d'immigrés nés dans le pays et parmi les enfants de personnes nées dans le pays, par genre, 20-29 ans, autour de 2007}

Hommes

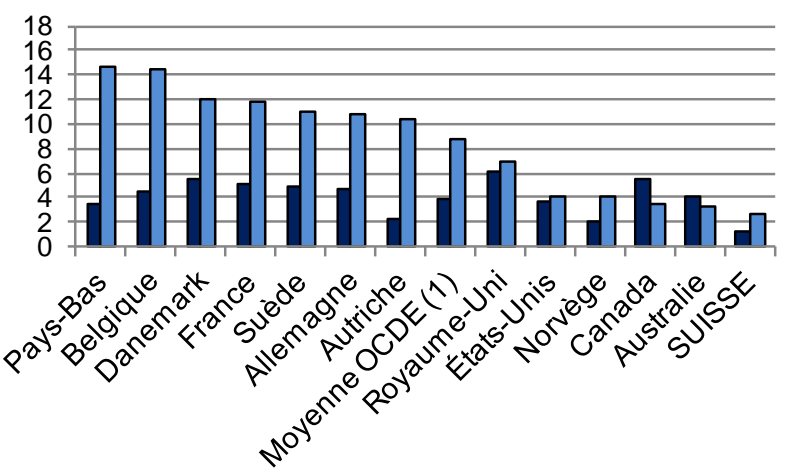

Enfants de personnes nées dans le pays
Femmes
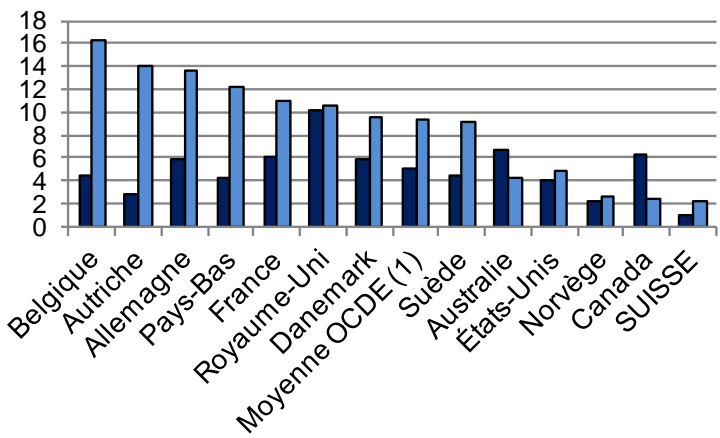

¿Enfants d'immigrés, nés dans le pays

Note : La "population à risque" est définie comme étant celle ayant un bas niveau d'éducation, sans emploi, ni scolarisée, ni en formation. 1 : La moyenne OCDE se réfère à la moyenne non pondérée des pays présentés dans le graphique.

Source: Adapté d'OCDE (2010a).

Les chiffres relatifs au groupe NEET pour les enfants nés en Suisse de parents issus de pays à faible revenu sont inférieurs au seuil de publication. 
19. Dans tous les pays, les enfants de parents issus de pays à faible revenu ont généralement un taux d'emploi inférieur à celui des enfants d'immigrés issus de pays à revenu élevé. Si quelque $90 \%$ des enfants d'autochtones et des enfants nés en Suisse de parents originaires de pays à revenu élevé occupent un emploi, le chiffre tombe en dessous de $80 \%$ pour les enfants d'immigrés de pays à faible revenu.

20. De fait, l'intégration globalement réussie des enfants d'immigrés de cette tranche d'âge sur le marché du travail en Suisse est à relier en partie au constat que la proportion d'enfants dont les parents sont originaires de pays à revenu élevé est plus forte en Suisse que dans tout autre pays de l'OCDE étudié à ce jour. Dans les deux tiers des cas ou presque, les enfants d'immigrés du groupe des 20-29 ans qui sont nés en Suisse ont des parents issus d'un pays à revenu élevé, principalement l'Italie, l'Espagne et le Portugal. A eux trois, ces pays fournissent près de la moitié des immigrés nés à l'étranger de cette tranche d'âge. Seuls $30 \%$ des enfants d'immigrés nés en Suisse ont des parents originaires de pays à faible revenu, contre $65 \%$ en moyenne pour les douze pays de l'OCDE sur lesquels on dispose de données (voir OCDE, 2010a). En Suisse, les deux tiers de ces jeunes sont nés de parents originaires de l'ex-Yougoslavie ou de Turquie.

21. Toutefois, la composition du groupe des enfants nés en Suisse de parents immigrés évolue assez rapidement, comme l'atteste le graphique 5. Parmi ceux qui entrent aujourd'hui sur le marché du travail (âgés de 15 à 19 ans), la majorité ont des parents originaires de pays à faible revenu, et représentent plus de six pour cent de cette cohorte d'âge.

\section{Graphique 5 : Nombre et composition des enfants d'immigrés nés dans le pays, groupe d'origine et âge, 2008/2009}

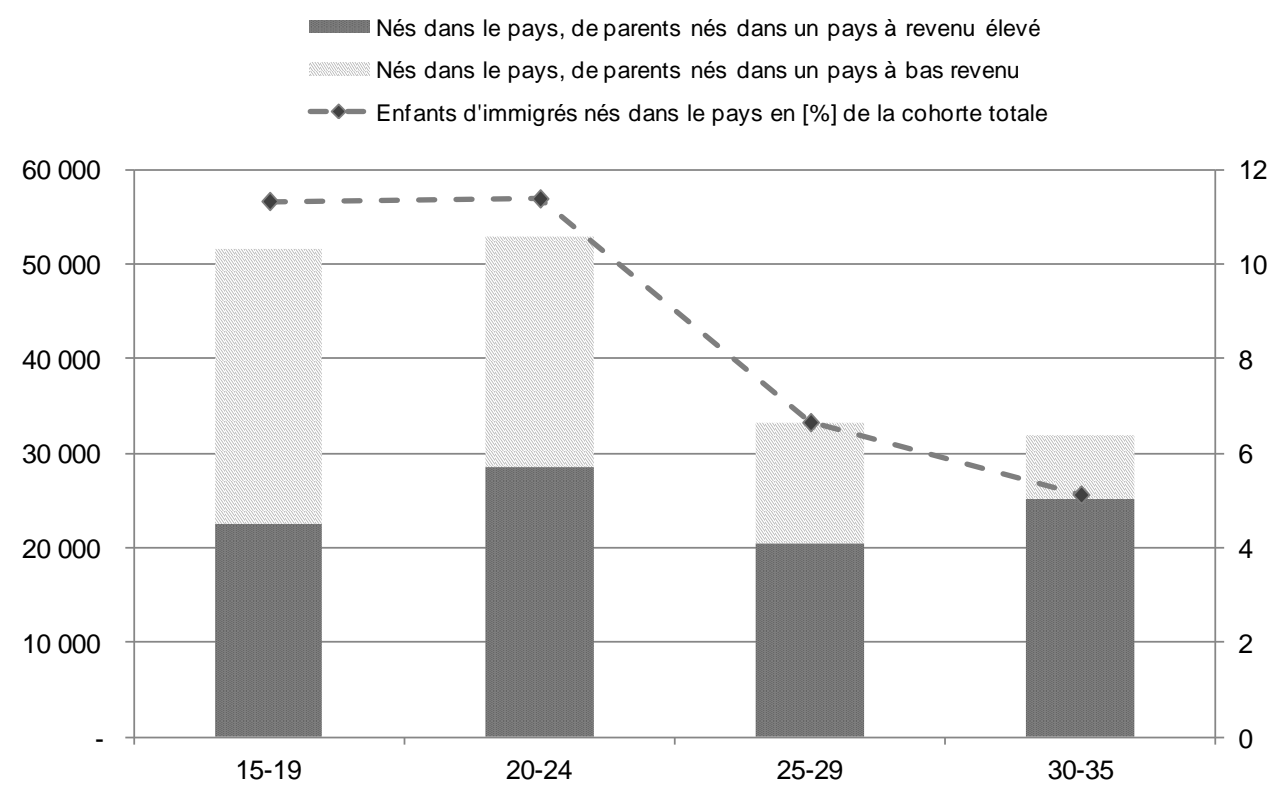

Source : Enquête suisse sur la population active, Office fédéral de la statistique, 2009.

22. En résumé, il ressort d'un premier examen des résultats d'ensemble que la composition favorable des pays d'origine de la population née à l'étranger joue un rôle déterminant pour expliquer les résultats globalement positifs des immigrés et de leurs enfants sur le front de l'emploi, en Suisse. Néanmoins, les résultats apparaissent tout à fait positifs même pour les immigrés - relativement peu nombreux originaires de pays à faible revenu qui rencontrent généralement davantage de difficultés dans tous les pays de l'OCDE. 
DELSA/ELSA/WD/SEM(2012)2

\section{LE CADRE D'INTÉGRATION}

\section{L'évolution des migrations à destination de la Suisse et les principaux groupes de migrants}

23. Peu après la Seconde guerre mondiale, la Suisse a connu un fort développement économique qui s'est traduit par des besoins accrus en main-d'œuvre. Le stock de capitaux du pays était resté quasiment intact, et le manque de bras s'est fait sentir plus tôt que dans les autres pays européens.

24. En 1948, la Suisse fut l'un des premiers pays d'Europe à recruter activement des travailleurs étrangers par le biais d'un traité avec l'Italie. A mesure que s'intensifiait la course aux «travailleurs invités » avec des pays comme l'Allemagne, la Suisse a dû revoir ce traité, en 1964, et accorder davantage de droits aux Italiens qui vivaient sur son territoire (par exemple, en assouplissant les dispositions relatives au statut de résident permanent et au regroupement familial).

25. Contrairement à d'autres pays d'Europe occidentale, il n'existait pas, en Suisse, d'organismes publics chargés du recrutement de travailleurs étrangers, lequel était organisé par les employeurs euxmêmes (voir Körner, 1990). Aujourd'hui encore, les partenaires sociaux (associations d'employeurs et syndicats) conservent, dans plusieurs secteurs, des agences de recrutement et de formation à l'étranger. La Suisse ayant d'abord considéré, comme d'autres pays, que ses besoins en main-d'œuvre et l'immigration qui en découlait seraient temporaires, de nombreux travailleurs immigrés se sont installés sur son territoire sans trop de restrictions. Toutefois, le développement rapide de ce vivier de main-d'œuvre s'est heurté à une hostilité croissante envers les migrants. En 1963, la Suisse a entrepris d'instaurer progressivement un système de contrôle de l'immigration de travail, et a ainsi plafonné le nombre d'étrangers par établissement.

26. En 1970, déjà, la proportion d'étrangers en Suisse était supérieure à $16 \%$ de la population, ce qui plaçait le pays, avec le Luxembourg, en tête des pays européens. ${ }^{7}$ Les trois quarts environ de la population étrangère provenaient alors des pays voisins. Les Italiens étaient de loin les plus nombreux (plus de la moitié de la population étrangère, voir le graphique 6), suivis par les Espagnols (11\%), les Allemands (11\%), les Français (5\%) et les Autrichiens (4\%).

27. La même année, le gouvernement suisse a fixé une limite supérieure globale au nombre d'immigrants autorisés à entrer dans le pays. ${ }^{8}$ La Suisse devenait ainsi l'un des premiers pays d'Europe occidentale à restreindre l'immigration de travail. C'est en partie grâce à cette réaction précoce qu'elle a pu maintenir le cadre initial en la matière, y compris après le premier choc pétrolier, et éviter, contrairement à d'autres pays de l'OCDE comme l'Autriche, la France et l'Allemagne voisines, de mettre un coup d'arrêt général au recrutement de la main-d'œuvre étrangère.

28. Lors du premier choc pétrolier, la Suisse n'avait pas instauré l'assurance-chômage obligatoire, ce qui poussa de nombreux étrangers dépourvus de protection sociale à quitter le pays. Les travailleurs étrangers ont ainsi joué un rôle d' « amortisseur » sur le marché du travail malgré une réduction de $8 \%$ de la population active totale - soit la baisse la plus forte au sein de l'OCDE - le taux de chômage n'a jamais atteint $1 \%$ (voir Sheldon, 2001).

On ne dispose de données concernant la population née à l'étranger que pour les années récentes. Pour l'essentiel de la période d'après-guerre, toutefois, les données relatives aux personnes étrangères et nées à l'étranger se recoupent largement.

8

Cette mesure a été prise en réponse à une initiative populaire ("Schwarzenbach") destinée à limiter à $10 \%$ la proportion d'étrangers par rapport à l'ensemble de la population. À l'issue d'une campagne intense, puis d'un vote marqué par l'un des plus forts taux de participation de l'histoire du pays et par un résultat relativement serré, la population suisse a rejeté cette initiative par $54 \%$ des voix contre $46 \%$. Cinq autres initiatives visant à limiter la proportion d'étrangers dans la population ont, depuis, fait l'objet d'un scrutin ; toutes ont été repoussées, la dernière en 2000. 
29. Le développement économique rapide de l'Italie et son intégration dans l'Espace économique européen, de même que le développement économique et la démocratisation de l'Espagne, ont rendu la Suisse moins attirante pour les Italiens et les Espagnols tentés par l'immigration. Pendant les deux décennies qui ont suivi le premier choc pétrolier, les besoins en main-d'œuvre de la Suisse ont été comblés, pour l'essentiel, par des ressortissants de l'ex-Yougoslavie et, dans une moindre mesure, du Portugal. Ceux-ci étaient recrutés principalement pour des activités saisonnières nécessitant peu de qualifications, dans les secteurs de l'agriculture et de l'hôtellerie. Au bout de quatre ans, cependant, les permis saisonniers pouvaient donner droit à un permis de séjour régulier (permis $\mathrm{B}$, délivré pour un an, renouvelable), la conséquence étant qu'un grand nombre de travailleurs immigrés sont entrés sur le territoire suisse par le biais d'emplois peu qualifiés dans des secteurs saisonniers. ${ }^{9}$ De fait, dans les années 1980, l'impact macroéconomique de l'immigration des années précédentes, majoritairement peu qualifiée, est devenu un important sujet de débat. Les données empiriques semblaient indiquer que cette immigration avait freiné les changements structurels et, partant, la croissance économique (voir, par ex., Schwarz, 1988). Parallèlement, la prédominance de l'Italie parmi les pays d'origine avait cessé : les immigrés récents étaient désormais plus nombreux à venir de l'ancienne Yougoslavie.

\section{Graphique 6 : Évolution et composition de la population étrangère en Suisse, nombres absolus et part dans la population totale, 1950-2009}
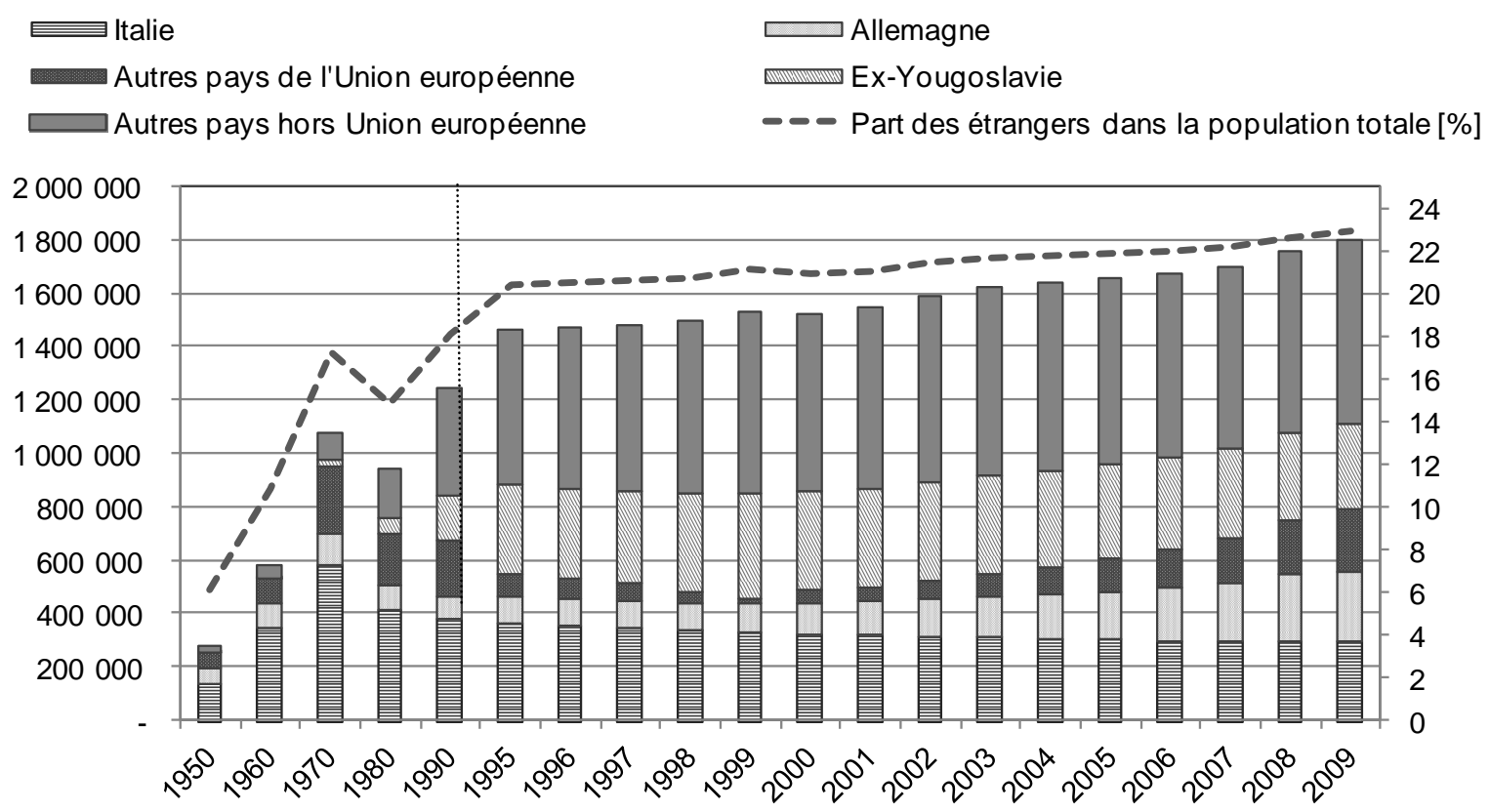

Note : Avant 1995, l'information est tirée du recensement suisse; À partir de cette année, les données viennent des registres de population.

Source : Calculs de l'OCDE basés sur les données de l'Office fédéral suisse de la statistique.

30. Face à une telle évolution, les autorités suisses ont lancé en 1991 le modèle dit « des trois cercles ». Selon ce concept, les migrants originaires de l'EEE bénéficiaient d'un traitement préférentiel (premier cercle). Si les besoins en main-d'œuvre n'étaient toujours pas satisfaits, il était possible de recruter des ressortissants des États-Unis, du Canada, d'Australie et de Nouvelle-Zélande (deuxième cercle). ${ }^{10}$ Tous les autres pays faisaient partie du troisième cercle. Parallèlement à l'évolution de la

Pour un examen de l'évolution de la politique suisse concernant l'immigration de travail, voir Liebig (2002). 
politique, l'immigration de travail en provenance de pays extérieurs à l'EEE a été progressivement limitée aux étrangers hautement qualifiés. Il est vrai que, par comparaison avec la situation des années 1980, les immigrés arrivés en Suisse dans les années 1990 étaient surreprésentés dans les emplois très qualifiés ; les faits montrent qu'une telle évolution a eu des répercussions positives sur la croissance économique de la Suisse (Becker, Liebig et Sousa-Poza, 2006).

31. Pays de longue tradition humanitaire, la Suisse constitue l'une des principales destinations pour les demandeurs d'asile. Jusqu'à la chute du Rideau de fer en 1989, toutefois, le nombre de demandes d'asile est resté relativement modeste, pour ensuite augmenter rapidement. Les demandes d'asile ont été particulièrement fortes dans les années 1990, décennie au cours de laquelle la Suisse a reçu, par rapport au nombre de ses habitants, plus de demandeurs d'asile que n'importe quel autre pays de l'OCDE. La majorité écrasante d'entre eux venaient de pays issus de l'ancienne Yougoslavie. L'année 1999 a été marquée par un pic en raison de la guerre au Kosovo : plus de 46000 personnes ont alors demandé l'asile en Suisse. Conséquence de ces flux de migrants humanitaires et du rapprochement familial, les trois quarts ou presque des immigrés originaires d'ex-Yougoslavie qui résident aujourd'hui en Suisse sont arrivés après 1991, c'est-à-dire après la fin des principales vagues de recrutement de travailleurs provenant de cette région. Ces immigrés récents sont établis, pour la plupart, dans la partie germanophone du pays, notamment en Suisse orientale (voir Annexe, graphique 4). Etant donné que les migrants humanitaires et leurs familles sont souvent moins bien placés sur le marché du travail, et ce dans tous les pays, il n'est guère surprenant que les taux d'emploi des personnes arrivées après 1991 restent sensiblement inférieurs à ceux des immigrés plus anciens de l'ex-Yougoslavie, quel que soit leur sexe. De même, l'incidence du chômage est plus forte pour cette catégorie de migrants (voir Annexe, tableau 3).

32. Aujourd'hui encore, la Suisse accueille, par rapport au nombre d'habitants, l'un des plus gros contingents de demandeurs d'asile de l'OCDE ; malgré tout, les chiffres actuels sont très en deçà de ceux des années 1990. La situation a évolué aussi en ce qui concerne les pays d'origine, parmi lesquels l'Afrique est désormais largement représentée.

33. Il n'en reste pas moins que l'immigration humanitaire occupe en général une place relativement modeste en Suisse par rapport à l'ensemble - elle a dépassé $10 \%$ du total de l'immigration de type permanent exclusivement dans les années 1990 (voir graphique 7). ${ }^{11}$ Comparée à la majorité des autres pays européens de l'OCDE ayant participé aux études de l'Organisation sur l'intégration des immigrés et de leurs enfants en matière d'emploi, la part des immigrés humanitaires dans les chiffres de l'immigration globale permanente s'avère plutôt limitée pour les six dernières années à propos desquelles on dispose de données standardisées (voir OCDE, 2011a). Pour ce qui est des demandeurs d'asile, en outre, la tendance apparaît plutôt anticyclique (voir graphique 8 ). ${ }^{12}$

été remplacé en 1998 par un système de recrutement dual, qui se borne à faire la distinction entre les ressortissants de l'EEE et les autres immigrants. Fondamentalement, ce modèle est toujours en vigueur aujourd'hui.

Il convient de noter que l'immigration familiale englobe, comme dans d'autres pays de l'OCDE, la fondation d'une famille et le regroupement familial, y compris les réfugiés.

On ne dispose de statistiques relatives à la main-d'œuvre que depuis 1991. 


\section{Graphique 7 : Évolution de la migration de type permanent en Suisse, par principale catégorie d'entrée, 1987-2009}

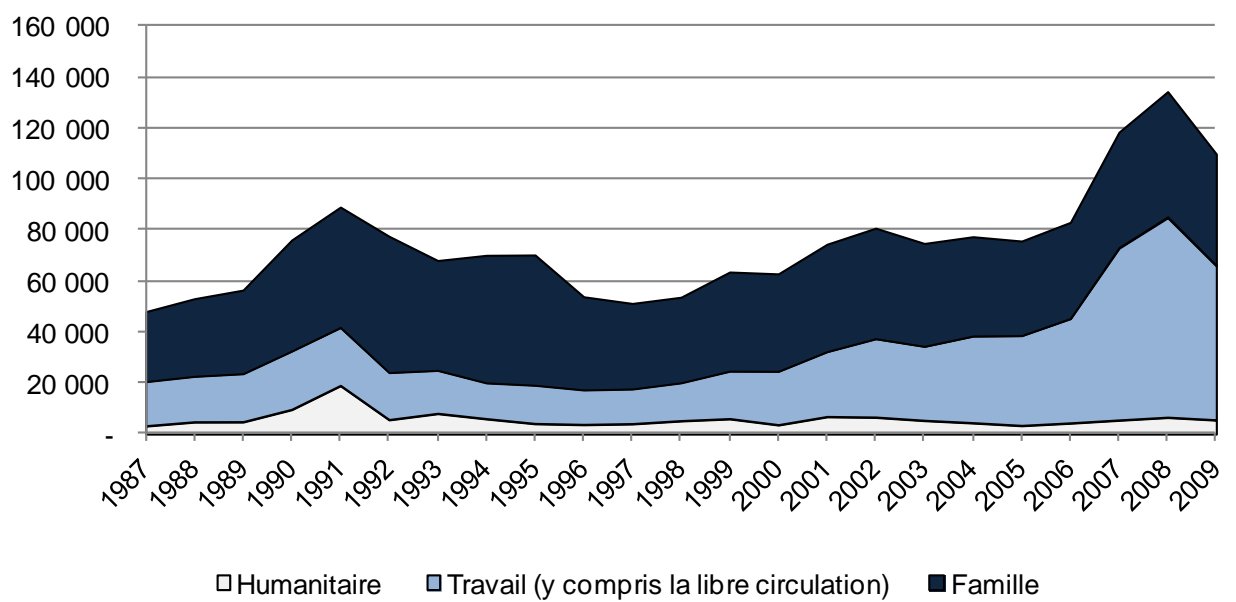

Note : Les retraités et les migrants venus pour étudier ne figurent pas dans le graphique. Les données sont basées sur les statistiques nationales et diffèrent légèrement des statistiques harmonisées publiées par l'OCDE (cf. OCDE 2011a).

Source : Office fédéral suisse de la statistique.

34. À compter de 2002, la Suisse a introduit graduellement la libre circulation avec les pays membres de l'EEE, ce qui s'est traduit, au cours des dernières années, par une explosion des migrations (de travail) dans ce cadre, surtout en provenance d'Allemagne. Cet afflux récent a modifié considérablement, de plusieurs façons, la composition de la population immigrée. ${ }^{13}$ Premièrement, la majorité des nouveaux travailleurs immigrés ont un bon niveau d'études, ce qui n'était pas le cas auparavant. ${ }^{14}$ Deuxièmement, alors que la proportion d'immigrés originaires de pays à revenu élevé était en recul depuis la fin des années 1980, elle s'est mise à augmenter de nouveau à partir de 2002. Enfin, la majorité des immigrés récents parlaient l'une des langues nationales de la Suisse à leur arrivée.

\section{Graphique 8 : Les demandeurs d'asile et la situation du marché du travail en Suisse}

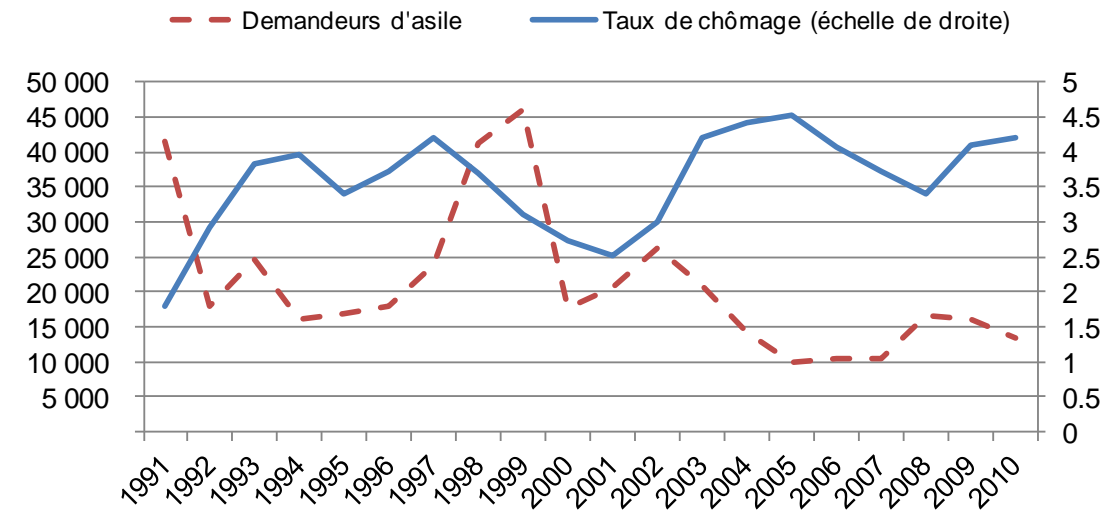

Source : UNHCR ; Statistiques de l'OCDE sur la population active.

Il ressort de l'Enquête suisse sur la population active qu'en 2009, 53 \% des immigrés originaires de l'UE-15 et installés en Suisse depuis moins de cinq ans avaient un diplôme de l'enseignement supérieur. 
35. Le graphique 9 donne un aperçu du volume et de la composition actuels des migrations de type permanent à destination de pays de l'OCDE. Le cas de la Suisse est particulier à deux égards. Premièrement, elle affichait en 2009 la plus forte proportion de migrants par habitant, avec un taux de $1.5 \%$ contre une moyenne OCDE de $0.6 \%$. Deuxièmement, les trois quarts des installations de type permanent relevaient pour la même année de la liberté de circulation, ce qui représentait la proportion la plus importante dans l'OCDE $^{15}$.

\section{Graphique 9. Flux permanents dans certains pays de l'OCDE et de pays non OCDE par catégorie d'entrée, 2009 (pourcentage de la population totale)}

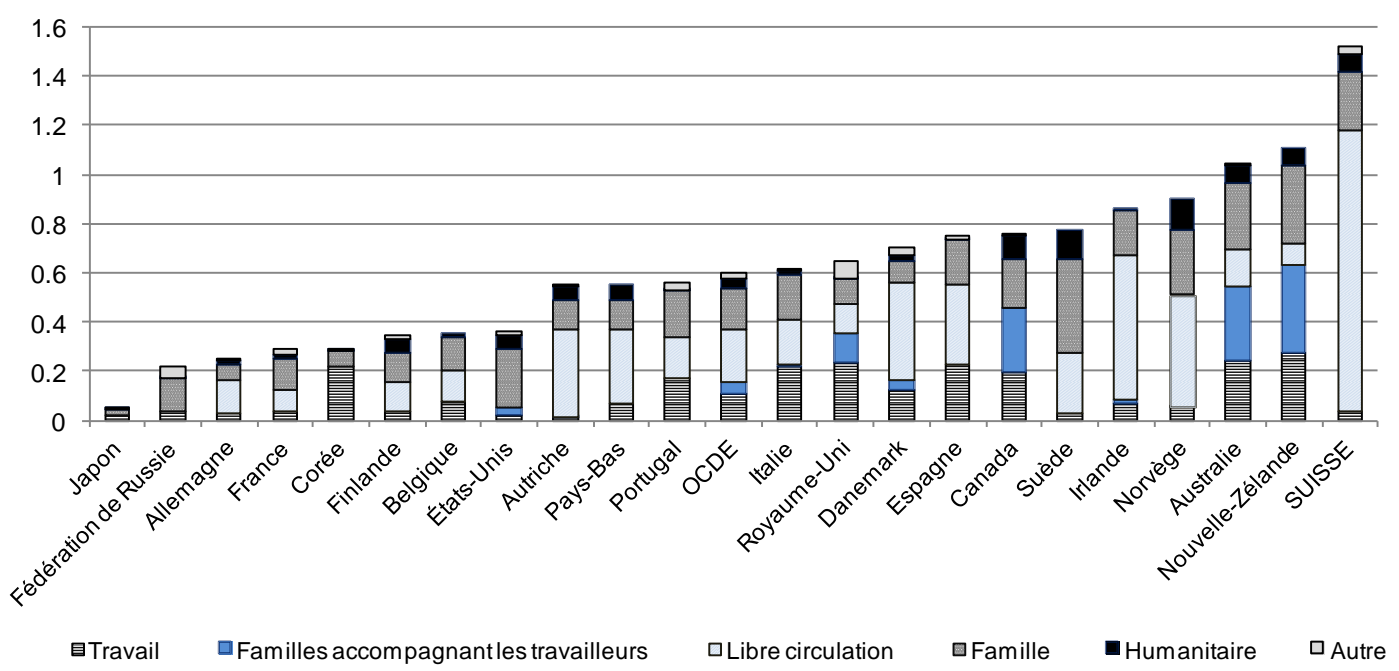

Note : Pour plus d'informations concernant le mode de calcul des statistiques standardisées, voir : www.oecd.org/migrations/pmi.

Source : OCDE, Perspectives des migrations internationales (2011b).

36. Sur l'ensemble de la population immigrée résidant en Suisse, plus d'une personne sur cinq en âge de travailler est issue d'un des pays de l'ex-Yougoslavie (voir Annexe, tableau 1). Le principal pays d'origine est l'Allemagne (13\%), suivie par l'Italie (10\%) et par le Portugal (9\%). Parmi les pays à faible revenu, la Turquie se classe en deuxième position après les pays de l'ancienne Yougoslavie, avec $5 \%$ du contingent total. L'encadré 2 donne, sous forme de comparaison internationale, une vue d'ensemble de la situation des immigrés venus d'ex-Yougoslavie ou de Turquie sur le marché du travail.

\section{Encadré 2. L'intégration des immigrés originaires d'ex-Yougoslavie et de Turquie sur le marché du travail en Suisse}

En Suisse, les immigrés provenant des divers pays issus de l'ex-Yougoslavie et de Turquie représentent $24 \%$ de la population immigrée en âge de travailler, et $54 \%$ des immigrés de cette tranche d'âge originaires de pays à faible revenu.

La base de données de l'OCDE sur les immigrés et les expatriés dans les pays de l'OCDE (DIOC-2005) ayant été actualisée récemment, il est désormais possible de comparer la situation des migrants sur le marché du travail en fonction de leur pays d'origine, dans les pays de l'OCDE où ils forment un contingent suffisant. Le tableau ci-dessous effectue cette comparaison pour sept pays européens de l'OCDE (vers 2005). Dans l'ensemble, la situation des deux groupes sur le marché du travail en Suisse est relativement favorable par rapport à ce qu'on observe dans d'autres pays. C'est particulièrement vrai pour les immigrées turques, dont le taux d'emploi atteint $59 \%$, contre une moyenne de $42 \%$ dans les sept pays. En revanche, les femmes originaires de l'ex-Yougoslavie dépassent à peine la moyenne, et font beaucoup moins bien qu'en Autriche et en Norvège. En outre, pour ces deux catégories d'origines et pour les deux sexes, on observe des écarts assez marqués dans les taux de chômage par rapport aux autochtones. Les différences sont particulièrement importantes dans le cas des hommes originaires de Turquie. 
DELSA/ELSA/WD/SEM(2012)2

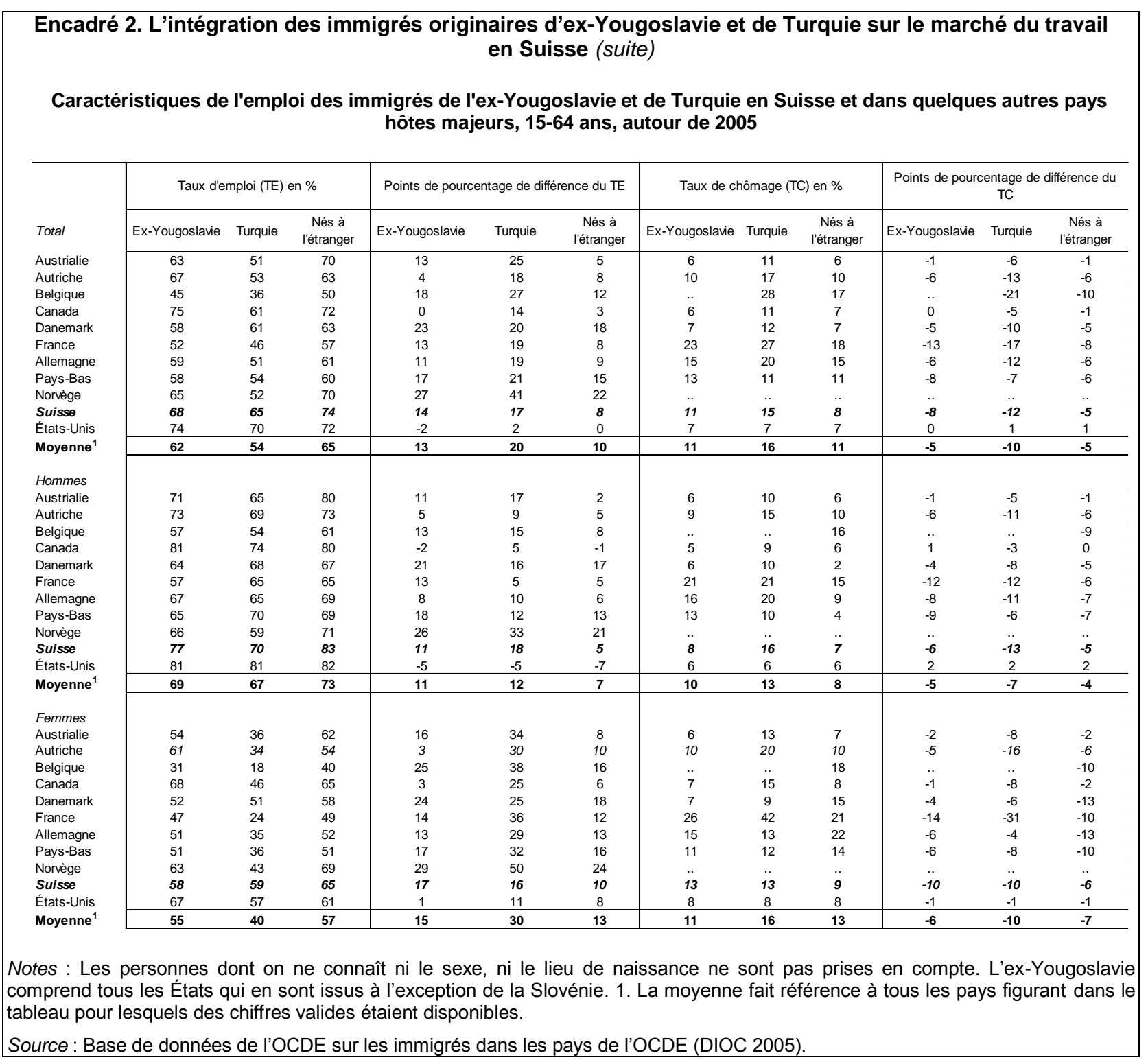

37. Comme dans d'autres pays de l'OCDE, la répartition des immigrés sur le territoire suisse n'est pas homogène. Le graphique 3 de l'Annexe fournit un panorama de la population immigrée, par région et en fonction de sa composition. La plus forte concentration (proche de $40 \%$ ) se trouve dans le canton italophone du Tessin où, de façon logique, la moitié des immigrés viennent d'Italie. Dans les autres régions de la Suisse, la population immigrée est plus diversifiée. Non loin du Tessin, on trouve de nombreux immigrés près du lac Léman, originaires principalement de la France voisine et du Portugal. À Zurich et dans le nord-ouest de la Suisse, les immigrés constituent aussi plus de $30 \%$ de la population. C'est le contingent allemand qui domine dans ces deux régions, suivi par celui des ressortissants de l'exYougoslavie.

38. En résumé, on peut distinguer quatre grandes vagues de migration vers la Suisse, de composition différente. La première, formée par les «travailleurs invités » et leurs familles - italiens pour la plupart est arrivée entre 1950 et 1970. La deuxième fut celle des derniers «travailleurs invités », venus entre 1970 et 1991, principalement d'ex-Yougoslavie, du Portugal et, dans une moindre mesure, d'Espagne. Sont entrés ensuite les migrants humanitaires, dont le nombre a connu un pic dans les années 1990 et demeure, 
aujourd'hui encore, non négligeable : si l'ex-Yougoslavie a d'abord été le principal pourvoyeur, les pays d'origine se sont récemment diversifiés, principalement au profit de l'Afrique, mais aussi de l'Asie. La quatrième vague, qui est également la plus récente, est liée à aux migrations au titre de la libre circulation, en provenance de l'Union européenne et, en particulier, d'Allemagne.

\section{L'évolution de la politique d'intégration}

39. Ainsi que nous l'avons vu précédemment, pendant la plus grande partie de l'après-guerre, la question de l'établissement durable de migrants sur le territoire et, par conséquent, toute idée d'intégration n'étaient pas à l'ordre du jour. La durée du séjour sur le territoire suisse était au contraire rigoureusement limitée, selon un système de rotation qui excluait le regroupement familial («Gastarbeiter »). Ainsi, aux termes de l'accord signé en 1948 avec l'Italie concernant le recrutement de main-d'œuvre, les travailleurs pouvaient être invités à tout moment à retourner dans leur pays, et il fallait résider en Suisse pendant dix ans avant de pouvoir obtenir un permis de séjour permanent. L'idée sous-jacente était que les immigrés n'aspiraient qu'à un séjour de durée limitée avant de regagner leur pays d'origine. La mobilité géographique sur le territoire était restreinte, de même que le droit à changer d'employeur. Ces restrictions à la mobilité sont restées en vigueur jusqu'en 2008, y compris pour les immigrés permanents.

40. Les importantes restrictions imposées aux immigrés en Suisse avaient pour principal objectif d'éviter un établissement durable. Quelques mesures ont bien été prises afin d'apporter un soutien aux travailleurs immigrés, mais elles visaient surtout à améliorer les conditions de leur séjour, et non à promouvoir leur intégration. ${ }^{16}$

41. Au début des années 1960, cette politique d'immigration axée sur les exigences du marché du travail, fonctionnant selon le principe d'une rotation des travailleurs sans se préoccuper de leur intégration, a commencé à montrer ses limites : un courant xénophobe est apparu dans l'opinion et des pressions internationales sont intervenues pour améliorer le statut des migrants. En 1953, déjà, l'OECE (qui deviendrait ensuite l'OCDE) avait suggéré que soit ramené de dix à cinq ans le délai requis pour l'obtention d'un permis de séjour permanent; par ailleurs, la diplomatie italienne s'employait activement à fournir une aide aux 400000 Italiens qui résidaient en Suisse. La Confédération céda du terrain sur ces questions et adopta de nouvelles directives facilitant le regroupement familial. Certains signes montraient, en outre, une prise de conscience accrue du fait que l'immigration était un phénomène durable et non pas simplement conjoncturel. Le Conseil fédéral chargea alors une commission d'étude formée de scientifiques et de représentants de l'administration d'élaborer un rapport exhaustif sur «le problème de la maind'œuvre étrangère ». Publié en 1964, ce document de planification définissait la voie à suivre pour les décennies suivantes : le modèle de rotation doit continuer à s'appliquer pour une majorité de migrants ${ }^{17}$ mais ceux qui sont appelés à rester en Suisse doivent emprunter - et se voir offrir - la seule issue qui permet de «lutter contre un excès de pénétration étrangère », à savoir l'assimilation puis la naturalisation (qui a été difficile à obtenir malgré tout : voir la section III, qui traite du sujet). La commission adopte, par ailleurs, une approche " universaliste », suggérant que l'égalité de traitement entre les citoyens suisses et les étrangers s'étende à toutes les formes de protection sociale et à la formation professionnelle. Elle s'élève aussi contre l'idée de scolariser les enfants d'immigrés dans des classes séparées.

16 En 1962, par exemple, il a été décidé de vérifier que les logements mis à disposition par les employeurs étaient adéquats. Cette mesure faisait suite à la constatation que les travailleurs étaient souvent mal logés, dans des baraquements ou des bâtiments vétustes convertis en logements. Le degré de ségrégation géographique des immigrés restait considérable et les contacts avec la population locale limités, sans que cela soit jugé préoccupant.

17 [le statut de saisonnier] "constitue l'un des moyens les plus efficaces contre l'Überfremdung et ... doit être maintenu dans toute la mesure du possible." (p. 200). 
42. Une stratégie plus ciblée a également été définie autour de la notion de "points de contact». Il s'agissait de créer dans les grandes villes des centres de soutien chargés de proposer différents services, par exemple des cours de langues, à l'intention des nouveaux arrivants. L'idée consistait à promouvoir une meilleure connaissance mutuelle et à limiter les conflits entre étrangers et autochtones. Cependant, la mise en œuvre de ce qui représentait à l'évidence le premier programme d'intégration jamais mentionné officiellement en Suisse fut laissée entièrement aux bonnes volontés individuelles : un appel en ce sens fut lancé implicitement aux propriétaires d'immeubles, colocataires, voisins, employeurs, collègues et subordonnés, membres d'associations et d'organisations en tout genre, artisans, directeurs d'organismes publics, médecins, enseignants, etc. Comme le veut la tradition suisse de partenariat social, les syndicats étaient perçus comme une très bonne filière d'intégration, ce qui rendait regrettable le fait que peu d'étrangers en soient membres. Il faut reconnaître que les différentes mesures concrètes adoptées le furent sans cohérence géographique ni coordination, offrant principalement une formation linguistique sommaire aux immigrés, ainsi que des conseils et un soutien aux employeurs, aux syndicats et aux associations représentant les immigrés aux niveaux national ou régional.

43. C'est seulement après le choc de l'initiative xénophobe «Schwarzenbach » du 7 juin 1970, qui proposait une réduction radicale du nombre d'étrangers et ne fut rejetée que de justesse, que les autorités fédérales ont pris davantage conscience de la nécessité d'intégrer les immigrés et de faire le nécessaire pour instaurer une politique concrète dans ce domaine. Très symptomatique à cet égard a été la mention, pour la première fois, de l'existence d'une «seconde génération » d'immigrants, lors d'une session du Conseil fédéral, en 1970 (Niederberger 2005). ${ }^{18}$ Une commission permanente spécialisée fut mise en place à l'échelle nationale - prédécesseur de l'actuelle Commission fédérale pour les questions de migration (voir plus bas). ${ }^{19}$ Si l'orientation universaliste des politiques à mettre en œuvre reste prédominante et si l'octroi à tous les travailleurs de la liberté de changer d'emploi, de profession et de résidence est visée par le programme du Conseil fédéral pour la période 1971-1975, la priorité nationale sur le marché du travail reste l'élément clé de la politique suisse et proroge, de fait, la politique de non-intégration des années 1960. Lorsque la situation économique est brutalement affectée par le choc pétrolier de 1973, le Conseil fédéral annonce ainsi que les demandeurs d'emploi suisses doivent avoir la priorité par rapport aux étrangers et une circulaire en ce sens est envoyée aux autorités cantonales (Piguet, 2009). Signe d'un changement en cours cependant : la Commission fédérale nouvellement formée prend la liberté de critiquer sur ce point les autorités fédérales.

44. Entre le milieu des années 1970 et les années 1990, période au cours de laquelle l'immigration s'est de nouveau accélérée, la politique fédérale d'intégration est demeurée minimaliste, se bornant à tenter d'améliorer progressivement la situation des étrangers au regard de la loi, de sorte qu'ils puissent jouir des mêmes droits civiques que les Suisses. Il s'est avéré que le principal obstacle à cette politique tenait à deux facteurs : le caractère sensible des questions d'immigration dans la population suisse et la volonté des mouvements xénophobes de recourir à la démocratie directe. Lorsque les mesures proposées avaient une portée universelle et ne concernaient qu'indirectement les étrangers, comme ce fut le cas avec la généralisation de l'assurance-chômage en 1976, elles étaient acceptées. Les initiatives plus ciblées, en revanche, ne passaient pas la barrière du scrutin populaire : on citera à ce propos la tentative de réforme de

"Pour cela, nos efforts doivent tendre avant tout à assimiler les jeunes étrangers de la seconde génération d'immigrants..." - Réunion du Conseil fédéral, extrait du protocole, mercredi 15 juillet 1970, Archives fédérales. auparavant, la ville de Zurich avait joué un rôle pionnier avec sa «Commission pour les questions d'assimilation » (Mahnig, 2005). La Commission fédérale fut ultérieurement baptisée «Commission pour les questions concernant les étrangers » en 1980, puis Commission fédérale des étrangers (CFE), en 1993 ; y siégeaient des représentants des employeurs, des églises, des syndicats, des communes et de la société civile en général. Le Conseil fédéral refusa jusqu'en 1980 d'admettre dans la Commission des représentants étrangers. 
la Loi sur les ressortissants étrangers, en 1978, ainsi que les deux tentatives visant à faciliter la naturalisation des enfants d'immigrés, en 1983 et $1994 .{ }^{20}$

45. Une autre raison explique que le gouvernement central n'ait pas joué un rôle très actif : il s'agit de l'importance que l'on attache en Suisse au principe fédéraliste de subsidiarité, particulièrement dans le domaine de l'intégration. En vertu de ce principe, c'est essentiellement aux autorités locales qu'incombe la mise en œuvre de mesures concrètes en faveur de l'intégration. Faute de bases légales, jusque récemment, pour affecter des ressources financières à l'intégration, la Confédération s'est trouvée réduite à tenter de stimuler l'action de cantons et de municipalités pas toujours convaincus de l'utilité des politiques proposées. La Commission fédérale s'est ainsi employée dans un premier temps, sans grand succès, à encourager la création de commissions locales chargées des questions d'intégration, qui serviraient de points de contact et centraliseraient les informations pour ensuite les disséminer (Niederberger, 2004). Elle a aussi voulu promouvoir l'implication, à titre consultatif, de délégués étrangers. En 1979, elle a publié un document en coopération avec les principales associations de communes suisses, dans le but de fournir informations et soutien aux municipalités dans le domaine de l'intégration, soulignant la nécessité de faire participer davantage les étrangers à la vie publique (Steiner, 2007). Révisé en 1989, ce manuel («Les étrangers dans notre commune ») montre bien le rôle prépondérant joué par l'échelon local en matière d'intégration jusque dans les années 1990.

46. Lorsque la Suisse a été confrontée, dans les années 1980, à une diversification accélérée de sa population étrangère et à l'accroissement du nombre d'enfants nés de parents immigrés, la Commission a entrepris d'inciter les cantons et les villes à instaurer des programmes de soutien scolaire à l'intention de ces enfants, à faciliter l'accès de ces populations aux services sociaux et médicaux et à veiller à ce que les parents puissent faire partie des conseils d'établissement. Parmi les mesures prises au niveau cantonal en faveur des immigrés, la création de classes séparées pour les nouveaux arrivants, d'abord décriée, s'est largement répandue. L'accent fut mis également sur la formation professionnelle des jeunes gens issus de parents immigrés, dont le nombre était en rapide augmentation.

47. Au cours des années 1990, l'immigration humanitaire s'est intensifiée ; les autorités fédérales ont alors accordé plus d'attention à la question de l'asile, au détriment des aspects liés à l'intégration. À cela est venu s'ajouter le fait que de nombreux retours ont été enregistrés lors de la récession de 1993, ce qui a renforcé l'idée selon laquelle les immigrés ayant du mal à s'intégrer sur le marché du travail finiraient par quitter le pays. En 1991 a été publié un important rapport commandé par le Conseil fédéral à ses services, qui exposait la conception et les priorités de la politique suisse à l'égard des étrangers pour la décennie (OFIAMT/OFE 1991), mais la question de l'intégration n'y était traitée que marginalement. C'est au niveau des villes et des cantons, grâce à l'introduction de politiques d'intégration actives, que le changement est devenu perceptible au cours de cette période. Bon nombre de villes et de cantons ont adopté des «lignes directrices » en la matière, et ont mis sur pied des services administratifs spécialisés (Sancar, 1999). ${ }^{21}$ Le rôle central joué ainsi par les instances locales confirmait le caractère prépondérant des structures « ordinaires » et « universalistes » en matière d'intégration (éducation, etc.), car ces dernières relèvent précisément, en Suisse, des compétences communales ou cantonales. Il est également possible que la forte décentralisation de la politique d'intégration ait contribué aux divergences, soulignées par maints observateurs, entre la conception de l'intégration en Suisse romande - davantage axée sur la participation et en Suisse alémanique, insistant plutôt sur les droits et les devoirs (D'Amato, 2009).

\footnotetext{
20 Une troisième tentative a échoué en 2004 (voir également la section III ci-dessous).

21 C'est à cette époque également que les cantons ont décidé de ne plus exclure du système scolaire les enfants dépourvus de permis de résidence, tout d'abord à Genève, en 1991, puis dans l'ensemble du pays, lors de la ratification par la Suisse de la Convention des Nations Unies sur les droits de l'enfant, en 1997.
} 
48. Vers la fin des années 1990, différents facteurs - la diversification accrue, déjà mentionnée, des pays d'origine et des motifs d'immigration, la perspective d'un abandon définitif du modèle de rotation lié au concept de «travailleur invité », la préoccupation suscitée par la situation socio-économique de certains groupes d'immigrés établis et la perspective d'une totale liberté de circulation au sein de l'Union européenne - ont donné à la question de l'intégration une place de toute première importance dans les programmes politiques. En 1999, un article sur l'intégration a été ajouté dans la loi sur les étrangers, conférant pour la première fois une base légale aux initiatives de la Confédération à l'appui des activités d'intégration des cantons. Le mandat général de la loi ainsi amendée a été précisé en 2000, avec l'entrée en vigueur d'une ordonnance du Conseil fédéral sur l'intégration des étrangers. La Confédération se voyait attribuer pour la première fois un mandat explicite, assorti d'une enveloppe de 10 millions CHF, afin d'œuvrer à l'intégration des immigrés. Certes, le montant était très modeste, mais une nouvelle ère s'ouvrait pour la politique d'intégration.

49. La nouvelle Loi sur les étrangers, entrée en vigueur en 2008, a élargi cette base légale. Elle comporte de nombreuses références à la question de l'intégration et confie à l'Office fédéral des migrations un rôle de coordination en la matière. Elle accorde en outre à la plupart des immigrés permanents l'accès au marché du travail et une totale mobilité dans le pays (c'est-à-dire le droit de changer de lieu de travail et d'employeur). ${ }^{22}$ De plus, les cantons ont désormais la possibilité d'adopter des conventions d'intégration, qui peuvent soumettre à certaines conditions, comme la participation à des cours de langue, à une formation professionnelle ou à tout autre programme censé favoriser l'emploi, l'octroi d'un permis de séjour ou l'accès à certaines prestations sociales pour les migrants originaires de pays non membres de l'UE/de l'AELE. À ce jour, cet outil n'a été utilisé que dans certains cas exceptionnels.

50. La nouvelle politique d'intégration de la Confédération s'énonce dans le «Rapport sur les mesures d'intégration» (Office fédéral des migrations, 2007), qui fixe les principaux objectifs de la politique fédérale d'intégration pour les années 2008-2011. Des mesures sont prises dans ce cadre afin d'augmenter les compétences interculturelles et linguistiques dans les organismes de médiation du travail, au moyen de stages et de recommandations ciblées, prévoyant par exemple de faire figurer les compétences interculturelles dans les critères de recrutement des nouveaux agents. En outre, la compétitivité des immigrés sur le marché du travail doit s'accroître avec l'acquisition d'un minimum de rudiments indispensables sur le marché du travail (comme les notions mathématiques utilisées dans la vie quotidienne), de même qu'avec une formation linguistique de base. La coopération entre agences gouvernementales, prestataires de services de placement et entreprises privées est à intensifier également, de même que doit augmenter le nombre de demandeurs d'emploi étrangers pouvant percevoir une aide à l'installation. En plus de ces mesures, qui font partie des services disponibles pour tout un chacun, la Confédération propose différentes initiatives spécifiquement axées sur les migrants (voir plus loin).

51. En 2010, le Conseil fédéral a décidé, dans le «Rapport sur l'évolution de la politique d'intégration de la Confédération », (Conseil fédéral, 21010 ; voir aussi de Coulon et Gäumann, 2011), de maintenir les niveaux de financement et les priorités actuels, tout en poursuivant la réorganisation des responsabilités. Le financement fédéral sera également renforcé à compter de 2014. La Confédération sera l'acteur stratégique, et les cantons des acteurs opérationnels, dans le cadre des politiques d'intégration. Bien que plusieurs débats parlementaires aient souligné que la discrimination constituait un obstacle de poids à l'intégration des immigrés sur le marché du travail, le Conseil fédéral n'a pas jugé nécessaire la mise en œuvre d'une législation supplémentaire pour lutter contre les mesures discriminatoires. Toutefois, plusieurs initiatives sont prévues, à partir de 2014, afin de susciter une prise de conscience. L’État fédéral

22 Avant l'adoption de la nouvelle loi, tout changement d'emploi ou de canton devait faire l'objet d'une demande d'autorisation lorsque la personne n'était pas titulaire d'un permis d'établissement ; en règle générale, celui-ci ne pouvait être obtenu qu'après cinq ou dix années de résidence en Suisse, selon que la personne était ou non originaire d'un pays de l'EEE. 
se propose, en outre, de rendre obligatoire, à cette même date, l'organisation de sessions d'information pour les nouveaux arrivants. Le potentiel d'intégration du migrant sera examiné lors de ces réunions et, suivant l'évaluation qui en sera faite et le statut d'admission de la personne, les autorités établiront avec elle une convention d'intégration.

\section{Les acteurs clés}

52. Nous avons vu plus haut qu'en raison du principe de subsidiarité et de la structure de l'État suisse, la politique d'immigration et d'intégration est relativement décentralisée dans le pays. Les programmes actifs du marché du travail étant dans l'ensemble décentralisés également, la coordination pose des difficultés bien particulières (voir Duell et al., 2010 pour une description exhaustive).

\section{Au niveau fédéral, plusieurs acteurs élaborent ou influencent la politique d'intégration}

53. L'Office fédéral des migrations (OFM) a été créé en 2005 pour traiter de toutes les questions migratoires relevant de compétences fédérales, notamment l'immigration et la naturalisation. Il est chargé de la politique fédérale d'intégration, ainsi que de la coordination des activités d'intégration au niveau des collectivités territoriales. Issu de la fusion entre l'Office fédéral pour les réfugiés et l'Office fédéral pour l'immigration, l'intégration et l'émigration, l'OFM compte plus de 700 employés. Il a progressivement développé, au cours des dernières années, ses activités dans le domaine de l'intégration : le budget prévu à ce titre, en 2010, avoisinait les 72 millions CHF. La plus grosse partie de ce montant -59 millions - se compose de paiements forfaitaires aux cantons, au titre de l'intégration des migrants humanitaires (réfugiés et personnes admises provisoirement sur le territoire). Seize millions CHF seulement ont été affectés aux mesures d'intégration générales (lesquelles ne ciblent pas spécifiquement les migrants humanitaires), essentiellement pour l'apprentissage de la langue (9 millions). Le solde était destiné à financer les "Centres de compétence pour l'intégration», dont la tâche consiste principalement à accueillir les migrants, à fournir des informations sur les questions d'intégration et à jouer un rôle de médiation entre les institutions et les personnes impliquées dans le processus d'intégration, ainsi qu'un «Centre de médiation interculturelle » et la mise au point de projets pilotes novateurs en matière d'intégration. D'ici 2014, il est prévu d'inclure dans le budget ordinaire les montants forfaitaires versés au titre des migrants humanitaires, et d'ajouter à l'ensemble 20 millions CHF. Cela devrait renforcer sensiblement non seulement le budget proprement dit, mais aussi le volume et la portée des mesures d'intégration au niveau fédéral, qui resteront néanmoins plus limitées que dans d'autres pays de l'OCDE.

54. Le deuxième acteur clé au niveau fédéral est le Secrétariat d'État à l'Économie (SECO), « centre de compétence » du gouvernement suisse pour toutes les questions relatives à la politique économique. Au sein du SECO, la Direction du travail est chargée de la politique liée au marché du travail. Le SECO effectue également un travail d'analyse de l'action des pouvoirs publics: il examine les politiques d'intégration sur le marché du travail, et rédige périodiquement des rapports assortis de recommandations.

55. La Commission fédérale pour les questions de migration (CFM) a été créée en 2008 par le Conseil fédéral, suite à la fusion de la Commission fédérale des étrangers (CFE) et de la Commission fédérale des réfugiés (CFR). Elle a pour fonction de faire le lien entre la société civile et l'administration fédérale. Instance extra-parlementaire, elle compte 30 membres élus pour un mandat de trois ans et emploie quatre personnes à temps plein. Son prédécesseur a assuré de 2001 à 2007 la gestion des fonds fédéraux spécialement affectés à l'intégration, d'un montant de 10 millions CHF par an. Depuis 2008, la CFM attribue chaque année, par adjudication, des aides représentant quelque 2 millions CHF. Les projets bénéficiaires peuvent porter sur l'apprentissage linguistique, sur les services d'intégration, ou sur des « innovations » dans lesquelles la Commission s'implique directement. Les fonds dont elle dispose étant limités, elle se concentre sur l'expérimentation, afin d'inciter les autorités locales à mettre en œuvre de bonnes pratiques. Outre le financement de projets, la CFM publie des rapports et des recommandations, et 
formule des avis à l'intention du gouvernement. En 2010-2011, elle a ainsi publié des recommandations sur plusieurs questions (femmes immigrées, contrats d'intégration, naturalisation, migrants sans papiers). Toutefois, l'intégration effective des immigrés sur le marché du travail n'est pas son premier domaine d'intérêt.

56. Il existe enfin, au niveau fédéral, une «Commission contre le racisme », dotée d'un budget assez modeste (155000 CHF en 2008). Cette instance a pour mission première l'organisation de campagnes d'information sur la discrimination et le racisme, et publie des déclarations de principe dans ces domaines.

\section{Qui dit « action concrète » dit « dépenses » : les responsabilités se situent alors au niveau cantonal/communal}

57. S'agissant des politiques et des dépenses liées à l'intégration, les responsabilités se situent pour la plupart au niveau des collectivités locales, mais sont réparties entre différents échelons et branches. Les cantons, par exemple, représentent le principal niveau administratif chargé des politiques actives d'intégration des chômeurs sur le marché du travail. Les offices cantonaux de l'emploi, qui font partie des départements économiques des cantons, gèrent les offices régionaux de placement, ORP/ " regionale Arbeitsvermittlungszentren", RAV. Les cantons se chargent également de l'assurance-chômage, mais l'administration des indemnités est en général dissociée des activités de placement. On soulignera, par ailleurs, que si l'assurance invalidité est régie par la loi fédérale, les offices y afférents sont gérés au niveau cantonal (pour plus de détails, voir Duell et al., 2010).

58. Outre les principales prestations fournies par le Service public de l'emploi (SPE), l'aide sociale et les offices d'assurance invalidité, la plupart des cantons ont instauré des services d'intégration, qui accompagnent des projets et leurs parties prenantes. Ils décident également des modalités de versement des sommes forfaitaires affectées à l'intégration des migrants humanitaires. Tous les cantons disposent aussi, désormais, de délégués à l'intégration chargés de coordonner les activités au niveau cantonal. L'ampleur et la portée de leurs tâches varient beaucoup, ce qui s'explique entre autres par les différences de volume et de composition de leurs populations immigrées (voir Annexe, graphique 3). Il n'est pas rare, même dans certains des plus grands cantons, que les activités d'intégration s'adressent plus ou moins exclusivement aux migrants humanitaires arrivés de fraîche date, auxquels on a vu que des fonds sont spécialement affectés.

59. Sous réserve de la réglementation propre à chaque canton, c'est généralement aux municipalités qu'incombent l'assistance sociale et l'aide à la santé. Les petites communes ont cependant la possibilité de regrouper leurs services d'administration des prestations sociales ou de les externaliser vers le canton, ce qui permet à ce dernier d'intervenir à tous les niveaux. Les politiques actives d'intégration sur le marché du travail visant les bénéficiaires de l'aide sociale sont le plus souvent cofinancées par le canton. Le SPE, dont le rôle est de placer les chômeurs et dont l'évaluation se fait sur ce critère, n'offre en principe pas de services aux bénéficiaires d'aides sociales dans le cadre des programmes actifs d'intégration sur le marché du travail. Les grandes villes ont elles aussi instauré leurs propres services d'intégration.

60. La Conférence tripartite sur les agglomérations (Tripartite Agglomeration Conference), fondée en 2001, sert de plateforme de coordination des politiques au sein des trois niveaux de gouvernement. L'une de ses principales tâches est de formuler des recommandations en matière d'intégration, et en 2009 elle a produit un document clef sur la future politique d'intégration (Tripartite Agglomeration Conference, 2009). Ce document contient des dispositions qui vont promouvoir le développement de la politique d'intégration (voir Conseil fédéral, 2010). 


\section{Le modèle opérationnel d'intégration sur le marché du travail fonctionne selon un schéma de rétribution pour services rendus et de contrats à court terme attribués à des entreprises privées, à vocation sociale ou non}

61. Les services publics de l'emploi jouent un rôle majeur en matière de placement, mais ce sont les acteurs privés qui accomplissent l'essentiel de cette tâche : on comptait en 2008 quelque 4000 agences privées de placement et de travail temporaire, qui assuraient le placement de 70 à $80 \%$ des demandeurs d'emploi pris en charge par le dispositif de placement institutionnel.

62. La plupart des fonds nécessaires aux politiques actives du marché du travail (PAMT) font l'objet d'une externalisation par les cantons. Les budgets cantonaux varient en fonction du volume de dossiers. Les organismes travaillant sous contrat assurent généralement la formation et la préparation. En ce qui concerne les prestataires de services intervenant dans le cadre des PAMT, ils peuvent être financés par différents cantons, et proposer des interventions à différents organismes de financement pour des bénéficiaires de prestations diverses. Quant aux instituts de formation - souvent étroitement liés à des associations d'employeurs - ils constituent un autre maillon clé du système suisse, le subventionnement des cours étant déterminé au vu du dossier du bénéficiaire.

63. Il semble que la diversité des acteurs intervenant dans la politique d'intégration des immigrés sur le marché du travail soit à la fois un atout et une faiblesse. D'une part, les acteurs ont la possibilité de tester différentes approches ; d'autre part, il est parfois difficile de reproduire une initiative fructueuse à cause de divergences sur les principes et la démarche à adopter, et les évaluations approfondies sont plutôt rares. Enfin, lorsqu'elle est externalisée, la mise en œuvre des PAMT est déterminée par les paramètres d'évaluation de l'offre, si bien que les initiatives peuvent être définies non pas en fonction des besoins de chaque bénéficiaire mais des objectifs de performance sur la base desquels un projet est approuvé. Il peut s'agir là d'un aspect problématique en ce qui concerne les migrants, qui ont des besoins particuliers et se trouvent fréquemment très éloignés du marché du travail. Cette situation pourrait notamment se traduire par une sorte d' " écrémage », les immigrés bénéficiant moins que d'autres des PAMT, encore qu'il existe à ce stade peu d'éléments à l'appui d'une telle supposition concernant la Suisse (voir la section III ci-après).

\section{Les organisations non gouvernementales (ONG) jouent un rôle plus limité qu'ailleurs}

64. Contrairement à d'autres pays de l'OCDE, comme la Belgique, l'Allemagne ou les pays scandinaves, il n'existe pas de représentation officielle des immigrés au niveau fédéral, et ce en dépit du fait que leurs représentants occupent la moitié des sièges à la Commission fédérale pour les questions de migration. Plus généralement, on observe que les ONG jouent un rôle moindre qu'ailleurs. En matière d'intégration, la principale ONG est l'Organisation suisse d'aide aux réfugiés (OSAR), qui conduit plusieurs projets liés à l'intégration au bénéfice des migrants humanitaires et des demandeurs d'asile. ${ }^{23}$ Son budget avoisinait les 6.8 millions CHF en 2010. 
65. À ce jour, les associations professionnelles et les associations d'employeurs n'ont pas joué un rôle majeur en ce qui concerne l'intégration des migrants sur le marché du travail. Les syndicats, au contraire, et en particulier les syndicats d'ouvriers, ont toujours eu un fort contingent d'immigrés parmi leurs membres. Plus de $50 \%$ des membres de l'UNIA, principal syndicat suisse, sont des immigrés (ce qui conduit ce syndicat à se définir comme «la plus grande association de migrants en Suisse »). L'UNIA propose des formations linguistiques, ainsi que des programmes de parrainage. Ceux-ci, toutefois, s'adressent surtout aux personnes qui occupent déjà un emploi. De plus, la forte proportion d'immigrés semble associée en grande partie à une concentration sectorielle (ainsi, la construction représente plus du quart des membres ayant un emploi) et comprend essentiellement des travailleurs immigrés (italiens, espagnols et portugais) installés depuis plusieurs décennies et, pour la plupart, proches de l'âge de la retraite, plutôt que de jeunes immigrés originaires d'autres pays ou des migrants humanitaires. 


\section{INTÉGRATION DES IMMIGRÉS SUR LE MARCHÉ DU TRAVAIL : PRINCIPALES PROBLÉMATIQUES}

\section{Qualifications et résultats en matière d'emploi}

66. La structure des qualifications de la population active suisse âgée de 25 à 54 ans est fortement dominée par des qualifications du deuxième cycle de l'enseignement secondaire et par des qualifications de l'enseignement supérieur (voir tableau 2). Comme aux États-Unis, en Autriche et en Allemagne, les personnes qui ont un niveau d'instruction intermédiaire représentent plus de $50 \%$ de la population autochtone ; en outre, les personnes très instruites représentent une proportion relativement forte, égale à $38 \%$. En revanche, la population peu instruite représente une proportion plus faible en Suisse que dans tous les autres pays du groupe de comparaison. Elle constitue en effet seulement $5 \%$ de la population autochtone, contre $15 \%$ en moyenne dans le groupe de comparaison.

Tableau 2. Distribution (en \%) des personnes nées dans le pays et des personnes nées à l'étranger, âgées de 25 à 54 ans, par niveau d'éducation, dans quelques pays à revenu élevé, autour de 2008/2009

\begin{tabular}{|c|c|c|c|c|}
\hline & & CITE 0-2 & CITE 3/4 & CITE 5/6 \\
\hline \multirow{3}{*}{ Autriche } & Nés dans le pays & 13 & 68 & 19 \\
\hline & Nés à l'étranger & 30 & 51 & 19 \\
\hline & Nés à l'étranger, pays à bas revenu & 44 & 43 & 14 \\
\hline \multirow{3}{*}{ Belgique } & Nés dans le pays & 23 & 41 & 37 \\
\hline & Nés à l'étranger & 39 & 30 & 31 \\
\hline & Nés à l'étranger, pays à bas revenu & 46 & 28 & 26 \\
\hline \multirow{3}{*}{ Danemark } & Nés dans le pays & 20 & 43 & 37 \\
\hline & Nés à l'étranger & 29 & 39 & 32 \\
\hline & Nés à l'étranger, pays à bas revenu & 42 & 36 & 22 \\
\hline \multirow{3}{*}{ France } & Nés dans le pays & 23 & 45 & 32 \\
\hline & Nés à l'étranger & 41 & 31 & 28 \\
\hline & Nés à l'étranger, pays à bas revenu & 43 & 30 & 27 \\
\hline \multirow{3}{*}{ Allemagne } & Nés dans le pays & 9 & 64 & 28 \\
\hline & Nés à l'étranger & 35 & 45 & 20 \\
\hline & Nés à l'étranger, pays à bas revenu & $\ldots$ & $\ldots$ & $\ldots$ \\
\hline \multirow{3}{*}{ Pays-Bas } & Nés dans le pays & 22 & 44 & 35 \\
\hline & Nés à l'étranger & 39 & 33 & 28 \\
\hline & Nés à l'étranger, pays à bas revenu & 45 & 32 & 22 \\
\hline \multirow{3}{*}{ Norvège } & Nés dans le pays & 18 & 44 & 38 \\
\hline & Nés à l'étranger & 26 & 35 & 38 \\
\hline & Nés à l'étranger, pays à bas revenu & 36 & 33 & 31 \\
\hline \multirow{3}{*}{ Suède } & Nés dans le pays & 11 & 55 & 34 \\
\hline & Nés à l'étranger & 25 & 40 & 35 \\
\hline & Nés à l'étranger, pays à bas revenu & 29 & 37 & 33 \\
\hline \multirow{3}{*}{ Royaume-Uni } & Nés dans le pays & 27 & 39 & 34 \\
\hline & Nés à l'étranger & 22 & 41 & 37 \\
\hline & Nés à l'étranger, pays à bas revenu & 26 & 38 & 36 \\
\hline \multirow{3}{*}{ États-Unis } & Nés dans le pays & 7 & 61 & 32 \\
\hline & Nés à l'étranger & 28 & 42 & 30 \\
\hline & Nés à l'étranger, pays à bas revenu & 31 & 41 & 28 \\
\hline \multirow{3}{*}{ Suisse } & Nés dans le pays & 5 & 58 & 36 \\
\hline & Nés à l'étranger & 27 & 38 & 36 \\
\hline & Nés à l'étranger, pays à bas revenu & 31 & 40 & 29 \\
\hline
\end{tabular}

Source : Enquête européenne sur la population active 2009 et, pour les États-Unis, Current Population Survey March Supplement 2009.

67. On rencontre davantage de personnes justifiant d'une qualification d'un niveau inférieur au deuxième cycle du secondaire parmi les migrants que parmi les personnes nées en Suisse - plus d'un quart des personnes nées à l'étranger étant peu instruites. En revanche, la proportion de personnes très instruites 
est aussi élevée que parmi les personnes nées en Suisse. Au sein du groupe constitué par les migrants originaires de pays à faible revenu, la part des personnes très instruites et celle des personnes peu instruites sont quasi identiques, s'établissant à environ $30 \%$ chacune.

68. S'agissant des résultats sur le plan de l'emploi, contrairement à ce qui est observé dans tous les autres pays européens membres de l'OCDE du groupe de comparaison, les immigrés qui ont un faible niveau de qualification sont dans une situation plus favorable que leurs homologues nés en Suisse (graphique 10) ${ }^{24}$. Toutefois, il existe un écart de taux d'emploi relativement grand entre les immigrés qui ont un niveau intermédiaire ou élevé et leurs homologues autochtones.

69. Parmi les immigrés très qualifiés, $69 \%$ occupent un poste correspondant à leur niveau de qualification, ce qui représente une proportion plus élevée que dans tous les autres pays (voir annexe, tableau 2). Toutefois, cette situation s'explique une fois de plus par le fait qu'un fort pourcentage de la population née à l'étranger est originaire de pays à revenu élevé. En réalité, ce groupe est même moins touché par la surqualification que la population autochtone. La situation est toute différente pour les immigrés originaires de pays à faible revenu, seules $53 \%$ des personnes très qualifiées originaires de ces pays occupent un poste qui correspond à leur niveau de qualification. Bien que cette proportion soit relativement élevée comparativement à ce qui est observé dans d'autres pays, elle est nettement plus faible que celle constatée parmi les personnes nées en Suisse ou les immigrés originaires de pays à revenu élevé, proportion qui s'établit respectivement à $72 \%$ et $76 \%$. Il apparaît donc que les immigrés très qualifiés originaires de pays à faible revenu rencontrent des difficultés pour faire valoir leurs qualifications et leur expérience professionnelle sur le marché du travail suisse, même si ces difficultés ne sont pas nécessairement plus grandes en Suisse qu'ailleurs.

\section{Graphique 10 : Points de pourcentage de différence entre les taux d'emploi des personnes nées à l'étranger et des personnes nées dans le pays, selon le niveau d'éducation, personnes âgées de 15-64 ans non scolarisées, moyenne 2008/2009}

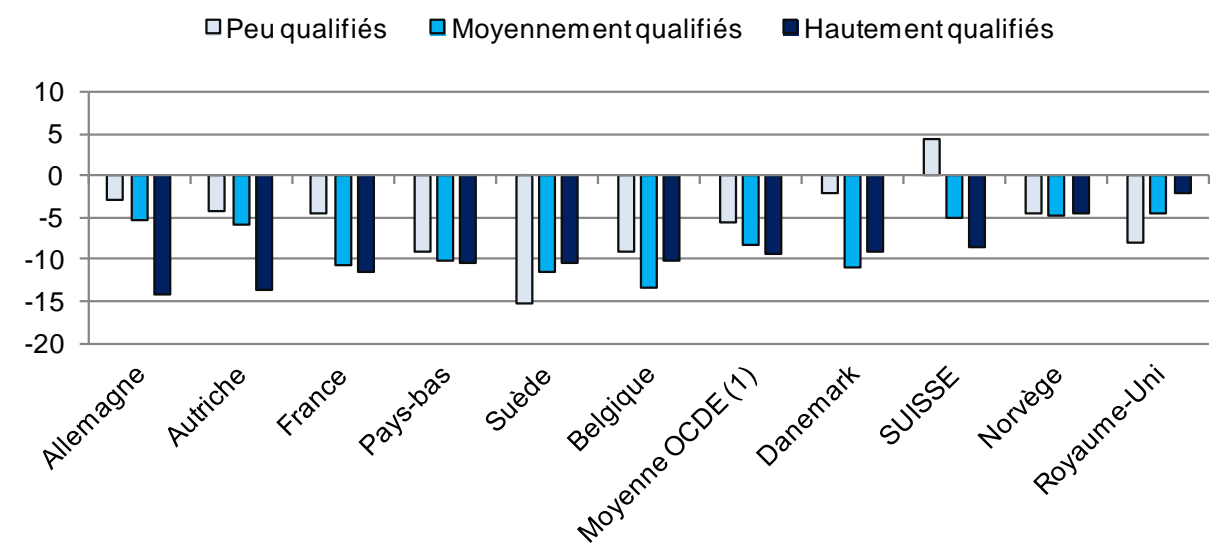

Note : La moyenne OCDE fait référence à une moyenne non pondérée de tous les pays figurant dans le graphique. Source : Enquête européenne sur la population active 2008-2009.

70. Plusieurs raisons peuvent être avancées pour expliquer ce constat. Premièrement, les immigrés peuvent avoir acquis leurs qualifications et leur expérience professionnelle à l'étranger ; or, ces diplômes et cette expérience peuvent être difficiles à évaluer par les employeurs, en particulier lorsqu'ils ont été acquis dans des systèmes éducatifs ou des contextes professionnels différents de ceux rencontrés en Suisse. Il est 
aussi possible que des qualifications acquises dans des pays non membres de l'OCDE soient en réalité moins recherchées sur le marché du travail suisse. De fait, certaines données montrent que ce phénomène explique en partie la décote des qualifications étrangères sur le marché du travail des pays de l'OCDE (voir OCDE, 2008b). Toutefois, il ressort d'analyses empiriques (tableau 3) que les migrants très qualifiés originaires de pays à faible revenu sont désavantagés même lorsqu'ils sont titulaires de diplômes suisses. Ce handicap reste significatif même si l'on tient compte des différences d'expérience professionnelle sur le marché du travail suisse. Il est permis d'en déduire que les migrants appartenant à ce groupe se heurtent à des obstacles supplémentaires indépendants du pays où ils ont acquis leurs qualifications ou leur expérience professionnelle.

71. Au nombre de ces obstacles pourraient figurer un accès insuffisant à des réseaux et une méconnaissance du fonctionnement du marché du travail, deux problèmes qui concernent particulièrement les migrants. Même si rien ne prouve que ces obstacles soient importants dans le cas de la Suisse, il est peu vraisemblable que la situation soit très différente de celle observée dans d'autres pays de l'OCDE, comme la Norvège, la Suède, l'Allemagne ou l'Autriche, où une grande partie - voire la plupart - des emplois supposent des contacts informels avec les employeurs. Les migrants ont moins de relations de ce type et certains pays de l'OCDE ont même mis en place des programmes de tutorat pour les aider à en tisser. Certains cantons suisses ont certes adopté de tels programmes, mais leur ampleur et leur portée sont limitées et ils s'adressent souvent à un autre public que les migrants ${ }^{25}$. La discrimination dans l'emploi reste le troisième obstacle possible et semble effectivement jouer un rôle important en Suisse (voir ciaprès).

72. La décote des qualifications étrangères est particulièrement forte pour les migrants qui ont obtenu leur diplôme le plus élevé dans un pays à faible revenu. Dans une analyse de données issues de l'Enquête internationale sur la littératie des adultes, Weins (2010) montre que la dévalorisation des titres étrangers sur le marché du travail suisse se vérifie même après prise en compte d'un indicateur objectif de la littératie. S'agissant des rendements de l'éducation, les migrants qui ont obtenu un diplôme professionnel ou universitaire à l'étranger tirent beaucoup moins avantage de leur investissement dans la formation professionnelle ou supérieure que les migrants qui ont obtenu un diplôme en Suisse. Ceux qui ont obtenu un diplôme à l'étranger (sauf lorsqu'il s'agit d'un diplôme professionnel de l'enseignement supérieur) ne semblent pas en retirer d'avantage significatif en termes d'amélioration de leur salaire par rapport aux personnes qui ne sont pas titulaires d'un diplôme du deuxième cycle de l'enseignement supérieur, alors que c'est le cas pour ceux qui ont obtenu leur diplôme en Suisse.

73. D'après les données de l'enquête suisse sur la population active (ESPA) sur la reconnaissance des qualifications, les immigrés qui ont fait officiellement reconnaître leur diplôme étranger ne sont plus réellement désavantagés sur le marché du travail suisse en termes de perspectives d'emploi (voir tableau 3$)^{26}$.

25 L'un des plus ambitieux de ces programmes est le programme de mentorat « Tandem » mis en place par le Canton de StGall pour faciliter l'intégration professionnelle des jeunes. Depuis 2006, 150 personnes en ont bénéficié.

En réalité, il semble même que leur situation sur le marché du travail soit plus favorable que celle des migrants originaires du même groupe de pays qui ont acquis leurs qualifications en Suisse. Toutefois, ce phénomène pourrait en partie s'expliquer par des différences de pays d'origine, de nombreux migrants appartenant à ce groupe et titulaires de qualifications suisses étant originaires d'Asie, d'Afrique et d'Amérique latine, tandis que ceux qui ont un diplôme étranger reconnu en Suisse sont plus souvent originaires d'Europe centrale et orientale. 
Tableau 3 : Points de pourcentage de différence de probabilité d'occuper un emploi hautement qualifié pour une personne ayant un niveau d'éducation élevé âgée de 15-64 ans en Suisse, personnes nées à l'étranger comparées aux personnes nées dans le pays, 2008

\begin{tabular}{|c|c|c|c|c|}
\hline Variables & Modèle 1 & Modèle 2 & Modèle 3 & Modèle 4 \\
\hline $\begin{array}{l}\text { Personnes nées à l'étranger } \\
\text { Plus haut niveau d'éducation obtenu en Suisse, dont: } \\
\text { Nés dans un pays à revenu élevé } \\
\text { Nés dans un pays à faible revenu } \\
\text { Plus haut niveau d'éducation obtenu dans un pays à revenu élevé } \\
\text { Plus haut niveau d'éducation obtenu dans un pays à faible revenu, dont: } \\
\text { Plus haut niveau d'éducation reconnu } \\
\text { Plus haut niveau d'éducation non reconnu, dont } \\
\text { N'a pas rempli un dossier de demande de reconnaissance des diplômes } \\
\text { Le dossier de demande de reconnaissance a été rejeté }\end{array}$ & $-6.5^{\star \star \star}$ & $\begin{array}{l}0.4 \\
-26.0^{\star * *}\end{array}$ & 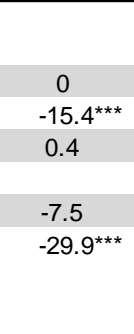 & $\begin{array}{c}-7.5 \\
-27.4^{\star \star \star} \\
-47.7^{\star \star \star}\end{array}$ \\
\hline \multicolumn{5}{|l|}{ Variables de contrôle } \\
\hline $\begin{array}{l}\text { Expérience professionnelle } \\
\text { Expérience professionnelle } \\
\text { Genre }\end{array}$ & $\begin{array}{l}0.5^{\star \star \star} \\
-0.02 \\
-6.0^{\star \star \star}\end{array}$ & $\begin{array}{l}0.6^{\star \star *} \\
-0.03^{\star \star *} \\
-5.6^{\star * *}\end{array}$ & $\begin{array}{l}0.6^{\star * *} \\
-0.03^{\star * *} \\
-5.7^{\star * *}\end{array}$ & $\begin{array}{l}0.6^{\star \star \star} \\
-0.03^{\star \star \star} \\
-5.7^{\star \star \star}\end{array}$ \\
\hline
\end{tabular}

Note : Les chiffres montrent la différence entre les immigrés et les personnes nées dans le pays. Ils correspondent aux effets marginaux obtenus par une régression logistique, calculée à partir des moyennes des variables dans les échantillons. Les personnes nées dans le pays constituent le groupe de référence. ${ }^{*},{ }^{* *},{ }^{* * *}$ indiquent une significativité aux seuils de $1 \%, 5 \%$ et $10 \%$, respectivement. Toutes les régressions comprennent des contrôles pour le genre et un indicateur des années d'expérience professionnelle en Suisse. Les dirigeants de petites entreprises (jusqu'à 5 employés) ont été exclus de l'échantillon.

Source : Enquête suisse sur la population active, Office fédéral de la statistique, Suisse.

74. Plus généralement on pourrait s'attendre à ce que la reconnaissance d'un diplôme étranger joue un rôle particulièrement grand en Suisse, dans la mesure où les titres officiels sont très importants sur le marché du travail et comptent beaucoup pour les employeurs pendant le processus de recrutement (voir Weins, 2010). Ce phénomène s'explique notamment par la stratification relativement forte du système éducatif suisse. Comme en Autriche, au Danemark et en Allemagne, il existe en Suisse des filières de formation professionnelle initiale et supérieure relativement spécialisées, qui débouchent sur la délivrance de diplômes officiels correspondant à des profils professionnels assez spécifiques (pour une étude plus approfondie du système de formation professionnelle initiale suisse, voir la partie IV).

\section{Reconnaissance des qualifications étrangères}

\section{Qualifications étrangères sur le marché du travail suisse et intérêt de la reconnaissance}

75. Étant donné que les migrants très qualifiés originaires de pays à faible revenu sont désavantagés sur le marché du travail suisse et que le fait de posséder une qualification étrangère officiellement reconnue semble offrir des avantages, on pourrait s'attendre à une forte demande de reconnaissance des diplômes, d'autant plus que plus de $80 \%$ des immigrés très qualifiés ont obtenu leur diplôme le plus élevé à l'étranger (voir tableau 4). Ils sont donc potentiellement touchés par la dévalorisation des diplômes étrangers de niveau supérieur sur le marché du travail suisse.

76. Il est donc étonnant de constater que dans leur grande majorité, les immigrés titulaires de diplômes de l'enseignement supérieur ne cherchent pas à les faire reconnaître. Seulement $25 \%$ environ des migrants originaires de pays à faible revenu déclarent avoir demandé la reconnaissance de leur diplôme, tandis que la majorité déclarent avoir jugé cette démarche «pas nécessaire » (voir tableau 4) ${ }^{27}$. Ceux qui

$27 \quad$ Il est toutefois difficile de déterminer s'ils choisissent cette réponse parce qu'ils n'ont tout simplement pas eu besoin de faire évaluer leurs qualifications pour trouver un emploi correspondant à leur niveau de compétences ou parce que leur qualification a été reconnue automatiquement, c'est-à-dire en vertu d'accords bilatéraux. La variable correspondante de 
déposent une demande ont de bonnes chances d'obtenir satisfaction puisque, jusqu'en 2008, sur trois demandes déposées par des migrants originaires de pays à faible revenu, deux recevaient une suite favorable. Ces constatations peuvent laisser perplexe - alors que la reconnaissance semble présenter un grand intérêt sur le marché du travail suisse, rares sont les immigrés qui cherchent à l'obtenir. Ce paradoxe pourrait s'expliquer, soit par une ignorance des possibilités de reconnaissance et de l'intérêt de cette démarche, soit par d'autres facteurs inhérents au système suisse de reconnaissance des diplômes étrangers de nature à dissuader les migrants de déposer une demande. Pour apporter un éclairage sur cette question, il faut commencer par examiner plus précisément ce système.

Tableau 4 : Origine des qualifications et participation aux procédures de reconnaissance des diplômes, personnes nées à l'étranger hautement qualifiées âgées de 15-64 ans, 2008

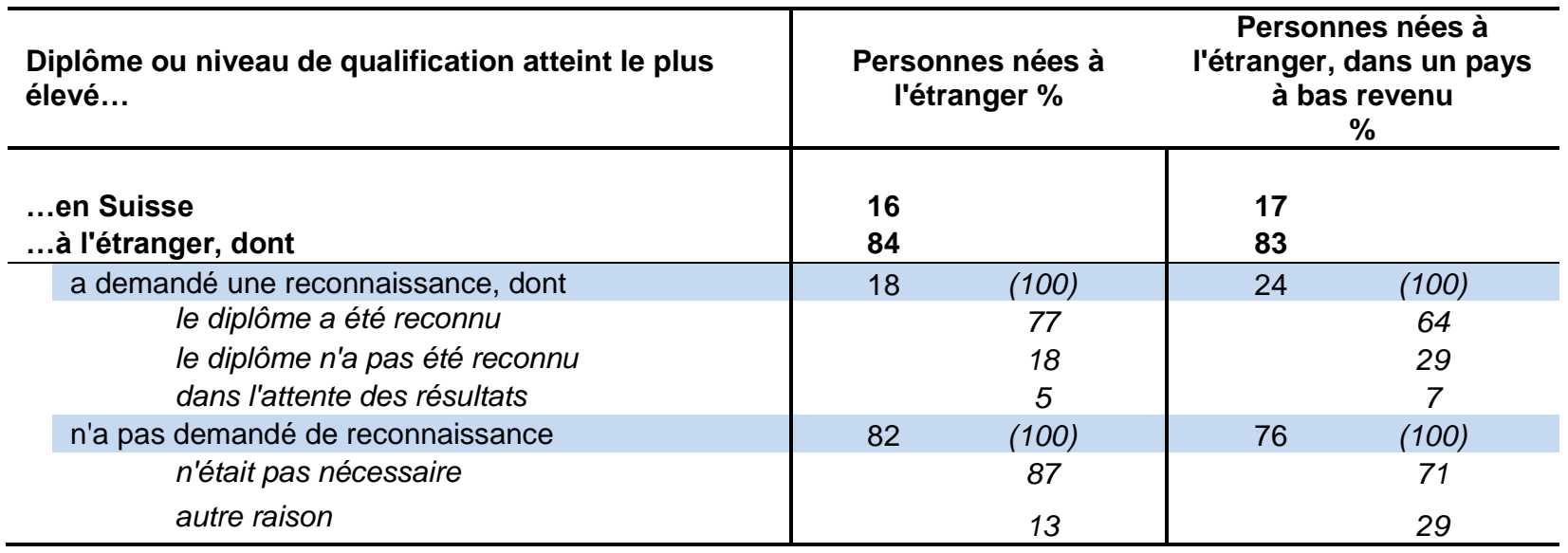

Note : Lecture : $84 \%$ des immigrés hautement qualifiées ont obtenu leur plus haute qualification à l'étranger. $18 \%$ ont déposé une demande de reconnaissance.

Source : Enquête suisse sur la population active, Office fédéral de la statistique, Suisse.

\section{Le système suisse de reconnaissance des qualifications acquises à l'étranger}

77. Comme dans d'autres pays de l'OCDE, il n'existe pas en Suisse d'obligation officielle de reconnaissance pour les professions non réglementées : il appartient à l'employeur d'accepter ou non les qualifications dont se prévaut le candidat. S'agissant des professions réglementées, une reconnaissance officielle est généralement exigée, mais une même profession peut être réglementée ou non selon la législation cantonale. Comparativement à d'autres pays de l'OCDE, il existe relativement peu de professions réglementées en Suisse, mais pour celles qui existent, le système de reconnaissance est complexe.

78. Il n'existe pas, au niveau national, de cadre ou de loi générale régissant la reconnaissance des qualifications étrangères, les compétences étant partagées entre de multiples acteurs, qui peuvent être des ministères et offices fédéraux, les autorités des cantons et des organisations telles que la Conférence des recteurs des universités suisses (CRUS) ou la Croix-Rouge suisse. Cette fragmentation est due au système fédéral et au caractère stratifié du système éducatif (les universités, les hautes écoles spécialisées et les programmes de formation professionnelle initiale relevant de la compétence de différents ministères et autorités cantonales), ainsi qu'au fait que certaines professions (comme les professions médicales) ne sont pas visées par les réglementations générales.

l'ESPA ne permet pas de faire une distinction entre différentes motivations, si bien que la réponse « inutile » peut avoir été choisie pour différents raisons. 
79. Le système de reconnaissance des diplômes le plus complet est géré par l'Office fédéral de la formation professionnelle et de la technologie (OFFT), chargé d'évaluer les qualifications professionnelles acquises dans le cadre de la formation professionnelle initiale, de la formation professionnelle supérieure et des hautes écoles spécialisées. Jouant en quelque sorte un rôle de guichet unique, l'OFFT reçoit les «demandes préalables» de reconnaissance et les soumet à un premier examen, destiné à identifier les diplômes qui relèvent de la compétence d'un autre organismee ${ }^{28}$. Le demandeur est ensuite informé des démarches qu'il doit accomplir et, si nécessaire, orienté vers l'organisme compétent.

80. S'agissant des demandes qui entrent dans son champ de compétence, l'OFFT procède à l'évaluation après réception des copies certifiées conformes des documents requis. La procédure d'évaluation dans son ensemble dure deux à quatre mois en moyenne. Lorsqu'une suite favorable est réservée à la demande, la reconnaissance peut revêtir deux formes. Pour les diplômes nécessaires à l'exercice de professions réglementées, l'OFFT délivre en général une reconnaissance (Anerkennung), qui coûte $550 \mathrm{CHF}$ et a une valeur juridiquement contraignante, dans le sens où elle autorise à exercer une profession réglementée. En cas de subsistance d'un doute sur l'équivalence entre le diplôme concerné et un diplôme suisse, l'OFFT peut recommander des mesures de compensation, mais ne propose pas de cours passerelles. Pour les diplômes correspondant à des professions non réglementées, pour lesquels une reconnaissance officielle n'est pas obligatoire, l'OFFT délivre une attestation de niveau (Niveaubestätigung), qui coûte 150 CHF. Cette attestation a pour objectif de situer le diplôme étranger au regard du système éducatif suisse pour aider les employeurs à en apprécier la valeur.

81. En 2009, l'OFFT a reçu environ 4000 « demandes préalables ». Un tiers seulement de ces demandes concernaient des professions réglementées, la majorité ayant été déposées par des migrants titulaires de qualifications correspondant à une profession non réglementée. Les demandeurs étaient, pour $60 \%$ d'entre eux, ressortissants de 1'Union européenne ${ }^{29}$. Environ 900 demandeurs ont été orientés vers d'autres organismes - par exemple la CRUS dans le cas des diplômes de l'enseignement supérieur - et 1200 demandes ont été évaluées et traitées par l'OFFT lui-même. Seulement 1 à $3 \%$ des demandes traitées ont été rejetées.

82. Les migrants qui ne peuvent pas apporter la preuve qu'ils sont titulaires d'un diplôme de formation professionnelle initiale (par exemple parce qu'ils ne disposent pas des documents requis) ou qui n'ont jamais obtenu de diplôme officiel du deuxième cycle de l'enseignement secondaire ne peuvent pas obtenir de reconnaissance officielle de l'OFFT. Toutefois, la Suisse met actuellement en place un système national de validation des connaissances acquises ("validation d'acquis »). Dans le cadre de ce système, les professionnels non titulaires d'un diplôme du deuxième cycle de l'enseignement secondaire auront la possibilité d'obtenir un diplôme de formation professionnelle initiale de base pour que les compétences formelles et non formelles qu'ils ont acquises dans le cadre de leur expérience professionnelle aient de la valeur sur le marché du travail ${ }^{30}$.

83. Les cantons sont chargés de la mise en œuvre de ce dispositif de validation d'acquis, tandis que l'OFFT édicte des lignes directrices à l'échelon fédéral. Pour améliorer l'harmonisation des procédures de

28 À ce stade de la procédure, l'OFFT n'exige pas de copies certifiées des diplômes. Cette évaluation se fait sur la base de documents (documents rédigés dans une des langues de la Suisse ou en anglais, ou traductions libres) décrivant le niveau, la durée, le contenu et la composante pratique du programme de formation.

Les qualifications acquises au sein de l'Union européenne ou de l'Association européenne de libre-échange (AELE) sont généralement reconnues dans le cadre de l'Accord bilatéral sur la libre circulation des personnes conclu entre la Suisse et l'Union européenne et ses États membres en 1999. Cet accord s'applique à une liste précise de professions réglementées qui est relativement longue et couvre à la fois des qualifications supérieures et professionnelles. 2002 et 2003. 
validation, l'OFFT a, en 2010, publié un guide en coopération avec les cantons, les partenaires sociaux et les organisations d'employeurs (voir Office fédéral de la formation professionnelle et de la technologie, 2010). Dans ce guide, les différents acteurs concernés élaborent une procédure d'évaluation par étape sur la base de profils de qualification individuels pour lesquels un diplôme officiel ne peut être décerné que si les candidats apportent la preuve qu'ils possèdent les compétences habituellement exigées des participants au cursus de formation professionnelle initiale correspondant. Bien que tous les cantons n'aient pas encore défini de procédure de validation, le processus est en cours.

84. Alors que la reconnaissance des qualifications professionnelles initiales est gérée relativement efficacement à l'échelon fédéral, les diplômes universitaires (à l'exception de ceux délivrés par les hautes écoles spécialisées) ne sont pas visés par les réglementations fédérales. Ils relèvent de la compétence de la CRUS, qui est habilitée à émettre des « recommandations » non contraignantes pour la reconnaissance des diplômes universitaires étrangers à condition qu'un cursus ou diplôme universitaire similaire soit proposé par une université suisse ${ }^{31}$. Ces «recommandations» visent à faciliter l'accès aux professions non réglementées et sont conçues pour les titulaires de diplômes étrangers désireux d'intégrer le marché du travail suisse.

85. Toutefois, lorsque la reconnaissance est une condition préalable indispensable pour suivre d'autres études supérieures, les universités évaluent elles-mêmes, de façon autonome, les diplômes antérieurs. Il en va également ainsi pour les certificats de fin de scolarité secondaire. À noter que les universités suisses tendent à être exigeantes quant aux pré-requis qu'elles demandent. Selon la CRUS - qui se réunit tous les deux mois avec les agents chargés des admissions dans les différentes universités suisses afin d'harmoniser les pratiques en matière de reconnaissance des diplômes -, les universités se montrent particulièrement méfiantes vis-à-vis des diplômes obtenus dans les pays à faible revenu. Il n'existe toutefois pas de conception commune susceptible de servir de base à la mise en place de cours passerelles destinés à combler les carences de la formation antérieure. Au contraire, les conseillers d'orientation ont tendance à ne pas orienter les candidats vers le système universitaire, ce qui est souvent justifié par l'idée que la Suisse offre des postes très qualifiés qui ne requièrent pas de diplôme universitaire.

86. En outre, des problèmes peuvent également se poser pour des diplômes universitaires obtenus en dehors de 1'Union européenne et correspondant à un diplôme de formation professionnelle initiale existant dans le système suisse (comme dans le cas du travail social). La CRUS ne peut pas émettre de « recommandation» pour ces diplômes et il appartient à l'OFFT d'évaluer s'il y a équivalence des diplômes. Or l'OFFT ne tient lui-même pas compte des diplômes universitaires (sauf de ceux délivrés par les hautes écoles spécialisées), même lorsqu'ils ont un diplôme de formation professionnelle initiale pour seul équivalent dans le système suisse. De ce fait, les migrants titulaires de ce type de diplôme ne peuvent bénéficier, ni des «recommandations » non contraignantes, ni d'une reconnaissance officielle de leur diplôme en Suisse. La validation d'acquis est donc particulièrement intéressante pour ces migrants, qui peuvent l'utiliser pour donner davantage de valeur à leurs compétences sur le marché du travail et pour obtenir une qualification officielle. Il en va de même des migrants qui se trouvent dans l'impossibilité de présenter des pièces justificatives de leurs études - ce qui est le cas, par exemple, de nombreux réfugiés.

87. Il existe des professions dont l'exercice est subordonné à l'obtention d'une autorisation officielle et qui sont par conséquent soumises à des procédures de reconnaissance spécifiques en Suisse. Il s'agit des professions exercées dans le domaine de la santé et de l'enseignement, de certaines professions techniques et des professions de juriste et de notaire. En l'espèce, la procédure de reconnaissance relève des autorités

31 Cette recommandation est formulée dans le cadre du Réseau européen des Centres nationaux d'information sur la reconnaissance et la mobilité universitaires (ENIC) du Conseil de l'Europe. La mise en œuvre de Swiss ENIC a été déléguée à la CRUS. La CRUS utilise les informations sur les correspondances recueillies et diffusées par ENIC et traite les diplômes obtenus en dehors de l'Union européenne en utilisant le même cadre. 
cantonales ou fédérales chargées de délivrer les autorisations d'exercice. En cas de compétence des autorités cantonales, la procédure de reconnaissance peut varier en fonction de la législation cantonale. La profession d'architecte, par exemple, est une profession réglementée nécessitant une reconnaissance officielle dans certains cantons et une profession non réglementée dans d'autres ${ }^{32}$. Les immigrés peuvent ainsi être désavantagés du seul fait qu'ils résident dans tel ou tel canton, ce qui est discutable du point de vue du respect du principe d'égalité de traitement, en particulier si leur situation au regard du droit au séjour ne leur permet pas de changer de canton facilement.

88. En outre, les migrants originaires de pays non membres de l'Union européenne et titulaires de diplômes médicaux font face à de nombreux obstacles sur le marché du travail suisse. Ces diplômes ne peuvent en effet être reconnus qu'en vertu de l'Accord bilatéral sur la reconnaissance réciproque des diplômes conclu entre la Suisse et 1'UE-25/AELE ${ }^{33}$. Par conséquent, les ressortissants de pays tiers, de même que les ressortissants bulgares et roumains titulaires de diplômes étrangers, ne peuvent pas bénéficier d'une reconnaissance officielle, sauf si leur diplôme a déjà été reconnu dans un État membre de l'Union européenne et satisfait à un certain nombre d'autres critères ${ }^{34}$. Certains cantons autorisent toutefois, dans certaines circonstances, les professionnels de santé formés à l'extérieur de l'UE/AELE à exercer même si leur diplôme n'est pas reconnu.

89. En somme, en Suisse, les procédures de reconnaissance des diplômes étrangers diffèrent sensiblement selon la nature et le niveau du diplôme, la profession, l'autorité chargée de délivrer les autorisations d'exercer et la législation cantonale. Cette disparité tend à rendre le système relativement complexe et va en général de pair avec une inégalité d'accès aux procédures de reconnaissance et à certaines professions, et elle entrave l'intégration sur le marché du travail des migrants titulaires de certains diplômes acquis dans les pays non membres de l'UE. Le coût des procédures n'étant pas élevé, la complexité du système et une méconnaissance de son fonctionnement et de son intérêt de la part des immigrés semblent être les principaux facteurs qui dissuadent ces derniers de l'utiliser davantage.

\section{Intégration sur le marché du travail des primo-arrivants}

\section{Tour d'horizon}

90. Ces dernières années, la Suisse a connu un afflux massif d'immigrés. Elle est le pays de l'OCDE qui a accueilli le plus grand nombre d'immigrés permanents depuis que l'OCDE a commencé à collecter des statistiques comparables, en 2003. De ce fait, environ un immigré résident sur cinq est arrivé au cours des cinq dernières années, ce qui représente $5 \%$ de la population résidente. Compte tenu de ces chiffres, la question de l'intégration de ces primo-arrivants sur le marché du travail revêt une importance particulière en Suisse.

91. L'idée de convergence qui sous-tend le concept d'intégration laisse entendre qu'à mesure qu'ils acquièrent le capital humain spécifique à leur pays d'accueil, en particulier les compétences linguistiques, les immigrés voient leurs résultats en matière d'emploi se rapprocher progressivement de ceux des autochtones. Les données relatives à d'autres pays de l'OCDE (OCDE, 2007a, 2008b) montrent qu'une

Autrefois, ces problèmes de mobilité intercantonale se posaient même pour les titulaires de diplômes suisses, mais la loi fédérale sur le marché intérieur suisse de 1995 a éliminé les obstacles auxquels se heurtaient jusqu'alors ces personnes.

L'accord vise à la fois les professions médicales de niveau universitaire (médecin, dentiste, vétérinaire et pharmacien) et les titres de niveau secondaire en médecine humaine et dentaire. La reconnaissance des professions médicales relève de la compétence de l'Office fédéral de la santé publique, tandis que celle des professions de niveau secondaire dépend de la Croix-Rouge suisse.

Pour des informations complètes sur cette règlementation, voir http://www.crus.ch/information-programme/anerkennungswiss-enic/reglementierte-berufe/gesundheitsbereich.html, 02.08.2011. 
insertion précoce sur le marché du travail joue un rôle fondamental dans les résultats en matière d'intégration à long terme.

92. Le graphique 11a présente, pour 2003/04 et 2008/09, le rapport emploi-population des immigrés en Suisse par nombre d'années de résidence et catégorie de migrants. Il en ressort que les primo-arrivants de sexe masculin affichent des résultats plutôt satisfaisants en matière d'emploi. Ces résultats sont particulièrement bons pour les migrants originaires de pays à revenu élevé, qui représentent la majorité des primo-arrivants. Ils sont également positifs par rapport à ceux constatés dans d'autres pays et restent bons même lorsque seuls les immigrés originaires de pays à faible revenu sont pris en compte (graphique 11b). Toutefois, la situation est moins favorable pour les femmes originaires de pays à faible revenu et certaines données montrent que les résultats des primo-arrivants se sont dégradés par rapport à ce qu'ils étaient.

Graphique 11a : Différences de taux d'emploi entre les immigrés et les personnes nées dans le pays, selon la durée de résidence, le groupe d'origine et le genre, personnes âgées de 15 à 64 ans, 2003/2004 et 2008/2009

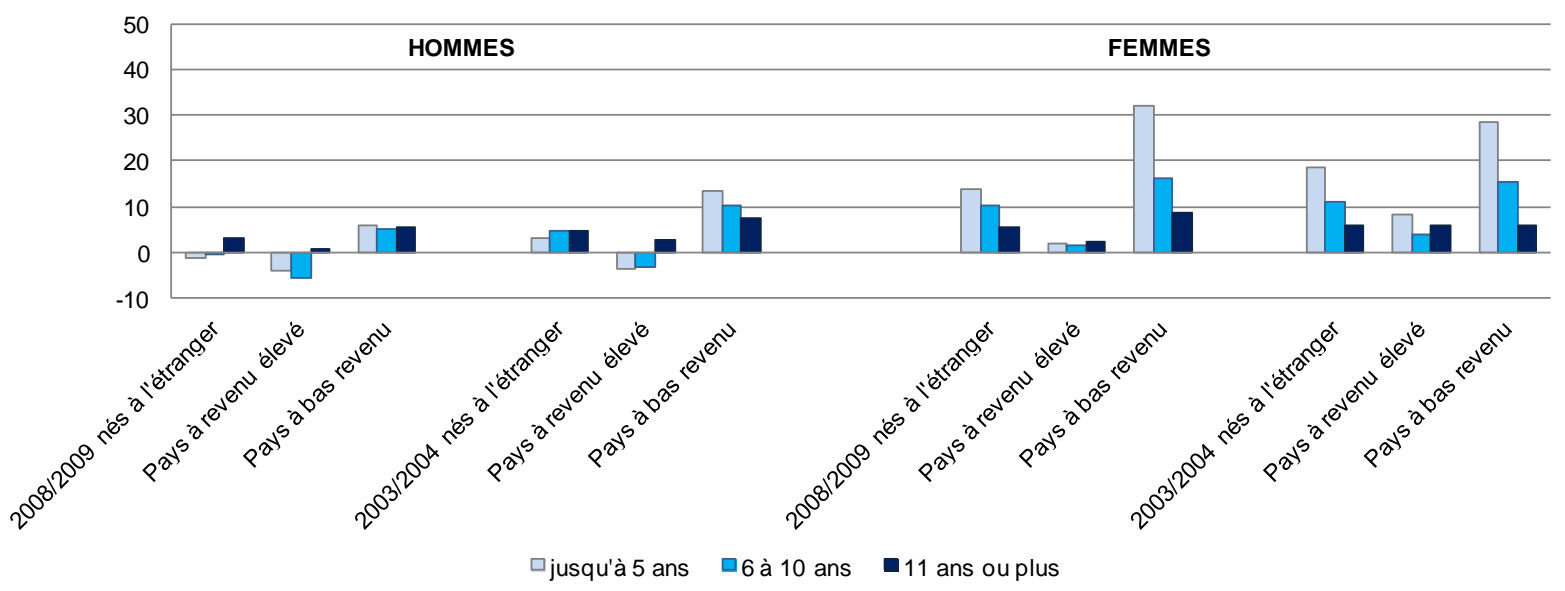

Source : Enquête suisse sur la population active, Office fédéral de la statistique, Suisse.

Graphique 11b : Différences de taux d'emploi entre les immigrés nés dans un pays à bas revenu et les personnes nées dans le pays, selon la durée de résidence et le genre, personnes âgées de 15 à 64 ans, dans quelques pays de l'OCDE, 2008/2009

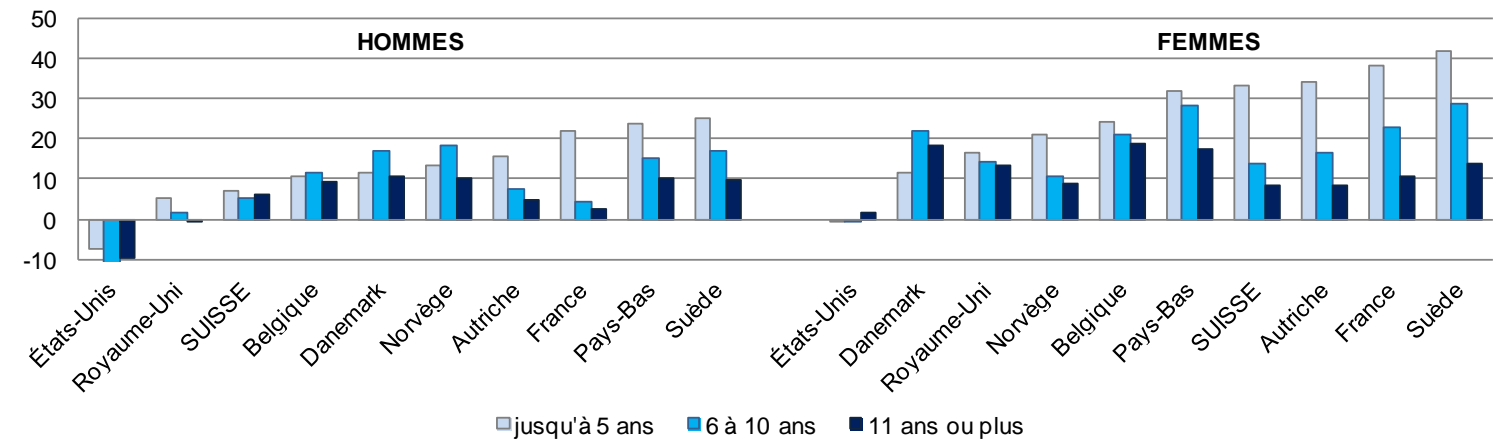

Note : La définition de "pays à bas revenu" diffère légèrement entre l'Enquête européenne sur la population active et l'Enquête suisse sur la population active. Les différences dans les taux d'emploi sont calculées comme le taux d'emploi des personnes nées dans le pays moins le taux d'emploi des personnes nées à l'étranger.

Source : Enquête européenne sur la population active. 
93. À noter que les graphiques 11a et $11 \mathrm{~b}$ ne reposent pas sur des données longitudinales, en d'autres termes que les individus ne sont pas suivis dans le temps. En utilisant les données de l'ESPA relatives à l'année d'arrivée, il est possible de procéder à une analyse de pseudo-cohorte pour les immigrés arrivés aux alentours de 2003. Les résultats de cette étude sont présentés sur le graphique 12. La situation des immigrés originaires de pays à faible revenu présente là aussi un intérêt particulier parce que la plupart d'entre eux ne sont pas venus en Suisse dans le cadre de l'immigration de travail - contrairement à leurs homologues originaires de pays à revenu élevé, en particulier de l'Union européenne (voir infra). En moyenne, le taux d'emploi des immigrés originaires de pays à faible revenu est faible au départ, mais on observe une convergence plutôt rapide au cours des trois premières années. Ainsi, après trois ans, $60 \%$ d'entre eux occupaient un emploi. Il s'agit là d'un taux d'emploi supérieur à celui observé pour l'ensemble des migrants pour cette durée dans des pays où la situation de l'emploi est aussi favorable comme le Danemark (environ $55 \%$ pour la cohorte arrivée en 2004), la Norvège (51\% pour la cohorte arrivée en 2002), l'Autriche (50\% pour la cohorte arrivée aux alentours de 2004) ou les Pays-Bas (40\% pour la cohorte arrivée en 2000). Toutefois, au-delà d'environ trois années, les progrès semblent marquer nettement le pas.

\section{Graphique 12 : Évolution estimée du taux d'emploi des immigrés qui sont arrivés autour de 2003, 15-64 ans}

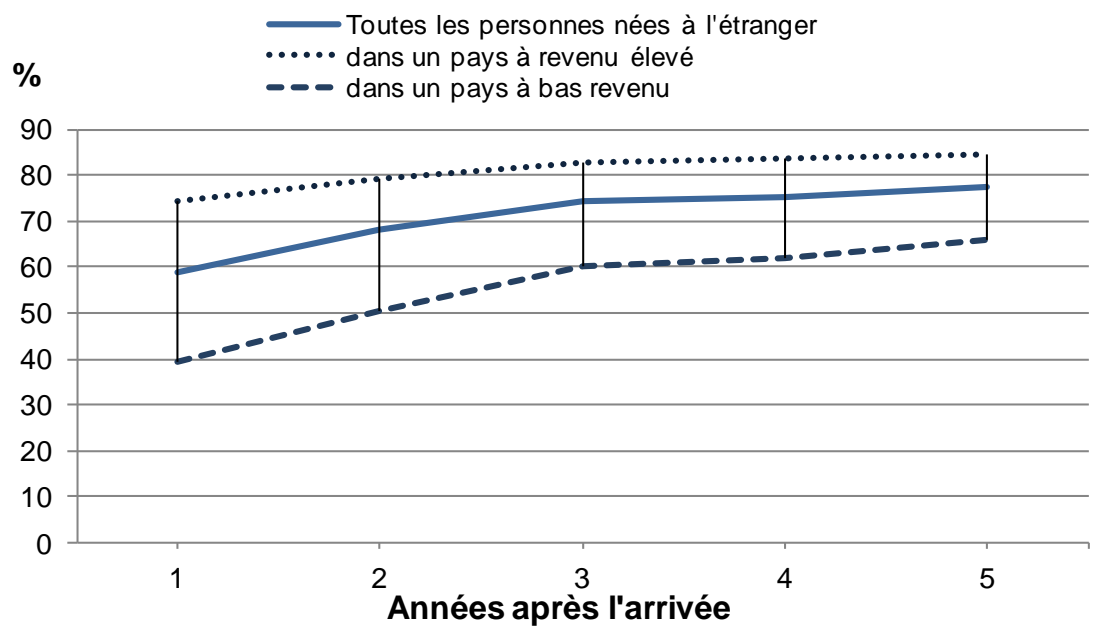

Note : Le graphique a été construit à partir de l'analyse d'une pseudo-cohorte en utilisant des données transversales et l'information sur les années de résidence. Pour obtenir des résultats solides, trois années consécutives de l'enquête sur la population active ont été regroupées.

Source : Enquête suisse sur la population active, Office fédéral de la statistique, Suisse.

\section{L'intégration des primo-arrivants originaires de l'UE-27}

94. La grande majorité - $64 \%$ - des primo-arrivants (qui résident en Suisse depuis moins de cinq ans) sont originaires de l'UE-27, leur arrivée s'inscrivant dans le cadre de l'instauration progressive, sur la base d'accords bilatéraux, de la libre circulation des personnes entre la Suisse et l'Union européenne et ses États membres. Les arrivées récentes représentent plus de $5 \%$ de la population active en âge de travailler, ce qui a été au cœur des débats publics en Suisse. Toutefois, ce n'est pas tant la question de leur intégration sur le marché du travail qui a fait débat que leur impact macroéconomique sur la croissance, les salaires, les prix de l'immobilier et les infrastructures ${ }^{35}$.

35 Les données disponibles montrent que cet afflux a eu un impact limité sur les salaires (voir, par exemple, Cueni et Sheldon, 2011). En revanche, il a apparemment eu un impact positif sur la croissance, tant au niveau global que par tête d'habitant. Il n'est pas possible d'établir avec précision dans quelle mesure l'immigration a exercé des pressions sur les infrastructures et 
95. La plupart de ces primo-arrivants venant pour travailler, il n'est pas surprenant que leur taux d'emploi soit supérieur à $84 \%$. Le niveau d'études élevé de ce groupe - $58 \%$ des primo-arrivants d'âge très actif (25-54 ans) sont titulaires d'un diplôme universitaire, contre $37 \%$ pour la population autochtone - explique également qu'ils parviennent dans l'ensemble à bien s'intégrer sur le marché du travail.

96. Le tableau 5 compare les résultats en matière d'emploi et le niveau d'études des primo-arrivants originaires de l'UE-27 à ceux de la population immigrée dans son ensemble et de la population autochtone. La conclusion la plus frappante est la forte disparité observée parmi les primo-arrivants originaires de l'UE élargie. Ainsi, la plupart des immigrés d'origine portugaise sont peu instruits et occupent des emplois peu ou moyennement qualifiés. Ils sont également confrontés à un chômage plutôt élevé. En revanche, les immigrés originaires d'Allemagne et de pays de l'Espace économique européen autres que l'Allemagne, le Portugal et l'Italie ont généralement un niveau d'instruction élevé et sont surreprésentés dans les emplois très qualifiés ${ }^{36}$. Ils affichent un taux de chômage similaire à celui de la population autochtone. Les primoarrivants originaires d'Italie se situent entre les Allemands et les Portugais en termes de niveau d'études et de résultats en matière d'emploi.

Tableau 5 : Résultats sur le marché du travail et profil d'instruction des récentes arrivées en Suisse, par pays, 15-64 ans, moyenne 2008/2009

\begin{tabular}{|c|c|c|c|c|c|c|c|c|}
\hline & \multicolumn{6}{|c|}{ Arrivées récentes de... } & \multirow{2}{*}{$\begin{array}{l}\text { tous les } \\
\text { migrants } \\
\text { résidents }\end{array}$} & \multirow{2}{*}{$\begin{array}{c}\text { nés dans le } \\
\text { pays }\end{array}$} \\
\hline & Allemagne & Portugal & Italie & autres UE-27 & tous UE-27 & $\begin{array}{l}\text { arrivées } \\
\text { récentes }\end{array}$ & & \\
\hline$\%$ des migrants résidents & 5 & 2 & 1 & 4 & 12 & 20 & 100 & - \\
\hline$\%$ des arrivées récentes & 26 & 11 & 5 & 22 & 64 & 100 & - & - \\
\hline \multicolumn{9}{|l|}{ Niveau d'éducation (en \%) } \\
\hline Bas & 3 & 73 & 20 & 6 & 17 & 19 & 29 & 15 \\
\hline Moyen & 36 & 19 & 34 & 29 & 31 & 32 & 39 & 57 \\
\hline Élevé & 61 & [8] & 45 & 65 & 52 & 50 & 31 & 28 \\
\hline \multicolumn{9}{|l|}{ Profil occupationnel (en \%) } \\
\hline Dirigeants & 5 & $\ldots$ & $\ldots$ & 7 & 5 & 5 & 3 & 4 \\
\hline Professionnels et techniciens & 65 & [9] & 52 & 65 & 41 & 50 & 37 & 45 \\
\hline Services, vente, artisanat et commerces associés & 19 & 57 & 27 & 18 & 28 & 27 & 33 & 27 \\
\hline Autres professions moyennement qualifiées & 9 & 16 & 12 & 8 & 13 & 11 & 17 & 21 \\
\hline Ouvriers et employés non qualifiés (ISCO 9) & [2] & 16 & $\ldots$ & [3] & 13 & 7 & 10 & 4 \\
\hline \multicolumn{9}{|l|}{ Résultats sur le marché du travail (en \%) } \\
\hline Taux d'emploi & 89 & 81 & 83 & 78 & 83 & 75 & 76 & 81 \\
\hline Taux de chômage & 3 & 11 & $\ldots$ & 5 & 5 & 8 & 7 & 3 \\
\hline $\begin{array}{l}\text { Taux de surqualification parmi les hautement et } \\
\text { moyennement qualifiés }\end{array}$ & 9 & $\ldots$ & 8 & 11 & 11 & 13 & 13 & 11 \\
\hline Salaire horaire brut médian (en CHF) & 41 & 26 & 32 & 45 & 38 & 36 & 35 & 40 \\
\hline
\end{tabular}

Source : Enquête suisse sur la population active, Office fédéral de la statistique, Suisse.

Note : Les chiffres entre parenthèses sont basés sur des échantillons en dessous de 50 . "..." se réfère à moins de cinq observations.

97. Cette situation globalement favorable se confirme même lorsque l'on examine d'autres indicateurs, comme les salaires (voir annexe, tableau 2). En moyenne, les primo-arrivants originaires de l'Espace économique européen ont un taux de surqualification inférieur à celui des autochtones. En outre,

le marché immobilier parce que ces pressions sont essentiellement dues à d'autres facteurs, indépendants de l'immigration (voir OCDE, à paraître).

36 Ces autres pays de l'Espace économique européen sont principalement la France, le Royaume-Uni et l'Autriche. 
une régression standard de la rémunération montre qu'à caractéristiques observables similaires, ils perçoivent des salaires légèrement plus élevés que les Suisses.

98. Cueni et Sheldon (2011) ont réalisé une étude complète des liens entre l'immigration récente en provenance de l'UE et le marché du travail suisse. Ils constatent aussi, qu'en moyenne, les migrants concernés occupent des postes plus qualifiés et sont plus rémunérés que les Suisses. Selon les auteurs, cet écart s'explique en grande partie (à plus de $75 \%$ ) par les caractéristiques observables plus favorables des immigrés arrivés récemment, notamment un niveau d'études plus élevé, une durée de travail plus longue et une surreprésentation dans les régions où les salaires sont élevés. En revanche, le risque de passer, d'une année sur l'autre, de l'emploi au chômage est quasiment $70 \%$ plus élevé pour les immigrés originaires de l'Union européenne que pour les autochtones, et un peu plus d'un tiers seulement de cet écart s'explique par des différences au niveau des caractéristiques observables, comme l'ancienneté. Enfin, les auteurs n'ont pas constaté d'impact négatif sur la rémunération des autochtones.

\section{Participation des immigrés aux mesures actives du marché du travail}

99. Comme indiqué précédemment, la Suisse a opté pour une approche reposant sur la prise en compte des questions d'intégration dans l'ensemble des politiques, si bien qu'il existe peu de mesures visant spécifiquement les immigrés. Toutefois, certaines mesures sont indirectement fortement ciblées, les immigrés et leurs enfants représentant la majorité des bénéficiaires de prestations de chômage et d'aide sociale $^{37}$. En avril 2011, une modification de la loi fédérale sur l'assurance-chômage obligatoire a limité les possibilités qu'avaient les services de l'emploi cantonaux de proposer certains dispositifs aux personnes ne justifiant pas de la durée de cotisation requise pour bénéficier de l'assurance-chômage, sauf si les services sociaux interviennent en leur faveur. De ce fait, il est désormais plus difficile pour beaucoup de primoarrivants et d'immigrés arrivés dans le cadre de l'immigration familiale d'accéder aux mesures du marché du travail. La participation à certaines mesures actives reste toutefois possible, à condition que les cantons contribuent au financement à hauteur de $50 \%$.

100. Bien que les immigrés soient fortement surreprésentés dans les groupes visés par les politiques actives du marché du travail, aucune étude n'a été réalisée pour évaluer leur participation effective à ces programmes ou pour apprécier l'impact effectif de ces mesures sur le groupe qu'ils forment ${ }^{38}$. De fait, alors que l'évaluation est une pratique courante en Suisse, les évaluations axées sur les immigrés restent rares. Toutefois, certaines évaluations utilisent la nationalité et/ou le statut au regard du droit au séjour comme variables de contrôle. Dans l'ensemble, la représentation des immigrés parmi les bénéficiaires de mesures actives du marché du travail est comparable à leur représentation parmi les chômeurs (voir Duell et al., 2010), ce qui avait déjà été constaté dans des études antérieures (voir Gerfin et Lechner, 2002 et Spycher et al., 2007).

37 Les immigrés et leurs enfants représentent aussi $35 \%$ des bénéficiaires du dispositif de prestations d'invalidité, qui constitue le troisième pilier du système de protection sociale. Pour une étude complète du système de protection sociale suisse et des politiques d'insertion, voir Duell et al. (2010).

Toutefois, Spycher et al. (2007) se livrent à une analyse approfondie des liens entre le système de sécurité sociale suisse et l'intégration des étrangers sur le marché du travail. De même, BASS (2006) dans une étude commanditée par le SECO examine les risques de chômage parmi les immigrés et discute des réponses politiques à y apporter. 
101. Dans leur évaluation microéconométrique des politiques actives du marché du travail mises en œuvre par la Suisse, Gerfin et Lechner (2002) constatent que les subventions salariales ont un impact positif pour les immigrés et que cet impact est plus fort que celui observé pour les ressortissants suisses présentant des caractéristiques comparables. Ils observent toutefois qu'étant donné leurs caractéristiques, les immigrés sont sous-représentés dans les dispositifs de subventions salariales temporaires ${ }^{39}$. Ces résultats sont confirmés par Lalive et al. (2002), qui constatent, en utilisant des données sur les titres de séjour, que ces dispositifs n'ont des effets très positifs que pour les étrangers titulaires d'un livret $\mathrm{B}$ (autorisation de séjour annuelle, renouvelable), mais que ces derniers sont nettement sous-représentés parmi les bénéficiaires de ces mesures. Ces résultats sont conformes aux données empiriques relatives aux pays nordiques (voir Nekby, 2008).

\section{Cours de langue}

102. De même, on dispose de peu d'informations sur les cours de langue proposés aux immigrés. L'Office fédéral des migrations consacre 9 millions CHF au financement de cours d'alphabétisation de base, qui ne représentent toutefois qu'une petite partie de l'ensemble des cours de langue offerts. Les cours sont principalement proposés par les cantons. L'offre est très diversifiée, variant non seulement entre les cantons mais au sein d'un même canton, du fait que la formation peut être financée par les services d'aide sociale, ou bien par les services publics de l'emploi ou encore par les services chargés de l'invalidité.

103. Il ressort des rares données disponibles que cette multiplicité d'acteurs va de pair avec une insuffisance plutôt qu'avec un excès de l'offre de cours de langue. Ainsi, dans une analyse des mesures destinées à faciliter l'intégration des immigrés sur le marché du travail, Spycher et al. (2007) constatent que les différentes branches de la sécurité sociale proposent trop peu de cours de langue et que la qualité générale de l'enseignement laisse souvent beaucoup à désirer.

104. Le module intégré à l'ESPA en 2008 fournit quelques données de base (autodéclarées) sur la participation aux cours de langue. Il en ressort que moins d'un immigré originaire de pays à faible revenu sur quatre a pu suivre un cours de langue dans les deux années qui ont suivi son arrivée. Moins de la moitié des personnes qui ont immigré en Suisse pour des raisons humanitaires déclarent avoir bénéficié de cours de langue, alors qu'elles forment le principal groupe ciblé par la politique d'intégration mise en œuvre à l'échelon fédéral (voir ci-après).

105. Bien que les cours de langue dispensés en Suisse n'aient jusqu'à présent fait l'objet d'aucune évaluation approfondie, des évaluations générales permettent de disposer de données de base sur l'impact des cours financés par les services publics de l'emploi. Dans l'ensemble, ces études révèlent que les perspectives sur le marché du travail des personnes qui suivent ces cours ne sont pas meilleures que celles des personnes qui ne les suivent pas. Gerfin et Lechner (2002) constatent même un impact négatif ; cela pourrait toutefois s'expliquer par une sélection négative des participants (à savoir que les immigrés qui rencontrent peu de difficultés ne participent pas) et cela n'implique pas nécessairement que ces cours aient effectivement un impact négatif. Quoi qu'il en soit, moins d'un tiers des participants trouvent un emploi dans l'année qui suit les cours.

106. Pour résumer, il existe une grande diversité de cours de langue en Suisse, mais il semble que seule une minorité des immigrés parlant une langue différente des langues officielles du pays en bénéficient et que les cours proposés ne soient pas fructueux en termes d'intégration sur le marché du travail. 
DELSA/ELSA/WD/SEM(2012)2

\section{L'intégration sur le marché du travail des migrants pour raisons humanitaires}

\section{Accès au marché du travail}

107. Le groupe formé par les immigrés arrivés en Suisse dans le cadre de l'immigration humanitaire se compose de deux sous-groupes de taille approximativement égale. Le premier, qui compte environ 25300 membres, est composé d'immigrés reconnus réfugiés aux termes de la Convention des Nations Unies. Ils obtiennent une autorisation de séjour (Livret B) et ont accès au marché du travail sans restriction. Le second groupe, qui comprend environ 23500 membres, est composé de personnes qui sont admises au séjour à titre provisoire et doivent demander un permis de travail, qui est toutefois accordé sans que la situation de l'emploi leur soit opposable (comme c'était le cas jusqu'en 2007), si elles trouvent un employeur prêt à les recruter ${ }^{40}$. En outre, lorsqu'elles veulent changer de canton de résidence, elles doivent en faire la demande. Ces dernières années, le nombre de personnes admises à titre provisoire a dépassé le nombre de primo-arrivants demandeurs d'asile.

108. Les demandeurs d'asile n'ont pas accès au marché du travail pendant les trois premiers mois. Audelà, ils peuvent travailler mais la situation du marché du travail peut leur être opposée. Il semble toutefois que la Suisse renonce en général à appliquer le critère de la situation de l'emploi pour certains postes peu qualifiés, pour lesquels il semble y avoir une pénurie de main-d'œuvre, en particulier pour les emplois dans le secteur de l'hôtellerie et de la restauration.

\section{Résultats en matière d'emploi par rapport à ceux observés dans d'autres pays}

109. Dans tous les pays de l'OCDE, les personnes qui immigrent pour motifs humanitaires rencontrent des difficultés particulières pour s'intégrer sur le marché du travail. Elles n'ont généralement pas de liens avec le marché du travail du pays d'accueil et souffrent souvent de difficultés psychologiques ou de handicaps. En outre, leurs qualifications et leur expérience professionnelle ont souvent été acquises dans des circonstances fort différentes de celles qui caractérisent le système éducatif et le marché du travail du pays d'accueil ; elles peuvent en outre être dans l'incapacité d'apporter la preuve de ces qualifications et de cette expérience.

110. Comparativement à ce qui est observé dans d'autres pays de l'OCDE, en Suisse les personnes qui immigrent pour des motifs humanitaires ne représentent qu'une faible proportion des personnes nées à l'étranger ${ }^{41}$. D'après l'ESPA qui, en 2008, comportait une question sur les motifs de l'immigration, ces migrants ne représentent que quelque $3.4 \%$ de l'ensemble de la population née à l'étranger et environ $10 \%$ des immigrés originaires de pays à faible revenu. En Suède, près de $23 \%$ des immigrés sont arrivés dans le pays pour des raisons humanitaires. En ce qui concerne les nouveaux flux d'entrée en 2009, d'après les statistiques normalisées de l'OCDE, moins de $5 \%$ des nouveaux migrants permanents, en Suisse, sont arrivés pour des raisons humanitaires, contre environ $10 \%$ en Autriche et $15 \%$ au Canada, en Norvège et en Suède.

Bien que ces migrants ne soient officiellement admis au séjour que provisoirement, il est vraisemblable que la plupart d'entre eux restent en Suisse. Ils ont toutefois un long chemin à parcourir pour obtenir un titre de séjour. Au terme d'une période de cinq ans, ils peuvent obtenir une autorisation de séjour (Livret B) qui peut être transformée en permis d'établissement après dix années supplémentaires.

L'analyse de la situation des migrants pour raisons humanitaires présentée infra repose sur les déclarations d'immigrés interrogés dans le cadre de la vague 2008 de l'ESPA (motif de l'immigration = pour demander l'asile). S'agissant des immigrés arrivés en Suisse ces 15 dernières années, on dispose également de données administratives (dites SYMIC). Une comparaison des informations sur les raisons de l'immigration figurant dans ces deux ensembles de données montre que, parmi les migrants qui ont déclaré être venus en Suisse pour des raisons humanitaires dans la vague 2008 de l'ESPA, $48 \%$ étaient recensés dans le système d'information central sur la migration (SYMIC) comme des migrants pour raisons familiales qui comprend également les migrations familiales concernant des migrants humanitaires. 
111. Le tableau 6 présente, sur la base d'informations fournies par l'ESPA, une comparaison des résultats en matière d'emploi des immigrés arrivés en Suisse pour des motifs humanitaires avec ceux des autres immigrés et des autochtones. Il en ressort que les personnes qui ont immigré pour des raisons humanitaires ont en général un niveau d'instruction plus faible que les autres migrants. Elles enregistrent néanmoins un taux d'activité relativement élevé, en particulier lorsqu'elles sont peu instruites. Les principaux problèmes d'intégration posés par ces migrants semblent être l'importance du chômage de longue durée et le fait qu'ils occupent souvent des postes pour lesquels ils sont surqualifiés. Ainsi, parmi les personnes très qualifiées arrivées en Suisse pour des raisons humanitaires, une sur deux environ occupe un emploi nécessitant une qualification inférieure à la sienne. Le taux d'emploi élevé est donc associé à une forte surqualification formelle. De fait, pour ce groupe, la question de la possibilité de transférer et de faire reconnaître les qualifications acquises à l'étranger est fondamentale (sur ces questions, voir supra).

\section{Tableau 6 : Résultats sur le marché du travail et niveau d'éducation des migrants pour raisons humanitaire en} Suisse, 15-64 ans, 2008

\begin{tabular}{|c|c|c|c|c|c|}
\hline & \multirow[b]{3}{*}{$\begin{array}{c}\text { Personnes } \\
\text { nées dans le } \\
\text { pays } \\
\text { (en } \%)\end{array}$} & \multicolumn{4}{|c|}{$\begin{array}{c}\text { Point de pourcentage de différence avec les personnes } \\
\text { nées dans le pays }\end{array}$} \\
\hline & & \multirow{2}{*}{$\begin{array}{c}\text { Immigrés } \\
\text { nés dans un } \\
\text { pays à } \\
\text { revenu élevé }\end{array}$} & \multicolumn{2}{|c|}{$\begin{array}{l}\text { Né dans un pays à bas } \\
\text { revenu }\end{array}$} & \multirow[b]{2}{*}{$\begin{array}{l}\text { Toutes les } \\
\text { personnes } \\
\text { nées à } \\
\text { l'étranger } \\
\text { (en \%) }\end{array}$} \\
\hline & & & $\begin{array}{l}\text { Migrants non } \\
\text { humanitaires } \\
\text { (en \%) }\end{array}$ & $\begin{array}{c}\text { Migrants } \\
\text { humanitaires } \\
(\text { en } \%)\end{array}$ & \\
\hline Pourcentage de tous les immigrés & & 54.3 & 42.2 & 3.4 & 100 \\
\hline Taux d'emploi & 81 & -1 & -11 & -4 & -5 \\
\hline quand le niveau d'éducation est bas & 56 & 16 & 3 & 20 & 10 \\
\hline quand le niveau d'éducation est élevé & 93 & -7 & -15 & -16 & -10 \\
\hline Taux de chômage & 2 & 2 & 6 & [8] & 4 \\
\hline Durée médiane du chômage (mois) & 4 & 2 & 5 & 11 & 4 \\
\hline Surqualifiés (2) & 21 & -8 & 8 & [28] & -2 \\
\hline $\begin{array}{l}\text { Pourcentage à plein temps parmi toutes les personnes } \\
\text { ayant un emploi }\end{array}$ & 65 & 7 & 10 & 16 & 9 \\
\hline $\begin{array}{l}\text { Salaire horaire brut médian pendant les douze derniers } \\
\text { mois des personnes ayant un bas niveau d'éducation et } \\
\text { travaillant à plein temps (CHF) ( } 3 \text { ) } \\
\text { Salaire horaire brut médian pendant les douze derniers } \\
\text { mois des personnes ayant un niveau d'éducation élevé } \\
\text { et travaillant à plein temps (CHF) (3) }\end{array}$ & 53 & 23 & 19 & -20 & 27 \\
\hline CITE1-2 & 15 & 10 & 21 & 24 & 15 \\
\hline CITE3-4 & 58 & -20 & -15 & -16 & -18 \\
\hline CITE5-6 & 28 & 10 & -6 & -8 & 3 \\
\hline
\end{tabular}

Note : 1. Les migrants humanitaires sont ceux qui sont entrés en Suisse avec l'intention de demander l'asile. 2. Uniquement les personnes avec un niveau d'éducation élevé $($ CITE5+6). 3. Le salaire horaire est dérivé du salaire brut total des répondants au cours des douze derniers mois en utilisant le nombre moyen d'heures travaillées par an en Suisse (1926h selon l'OFS). Seules les personnes ayant déclaré avoir été employées sans "interruption longue" sont prises en compte.

Source : Enquête suisse sur la population active, Office fédéral de la statistique, Suisse.

112. À noter que des données issues de l'ESPA présentées supra renvoient au premier titre de séjour obtenu par la personne lors de son entrée en Suisse, qui peut ne pas être le même que celui dont elle est titulaire au moment de l'enquête, en particulier pour les migrants qui résident en Suisse depuis plus de dix ans. Or $80 \%$ des migrants pour raisons humanitaires interrogés dans le cadre de l'ESPA résidaient en Suisse depuis plus de dix ans et $94 \%$ depuis plus de cinq ans. 
113. Pour étudier plus particulièrement l'intégration sur le marché du travail de cohortes d'immigrés plus récentes, l'Office fédéral des migrations a conduit en 2006 une grande enquête auprès des réfugiés qui venaient d'obtenir leur titre de séjour et auprès de deux cohortes supplémentaires de personnes reconnues réfugiées depuis un an, d'une part, et depuis trois ans, d'autre part. Ces cohortes enregistraient respectivement un taux d'emploi de $22 \%, 17 \%$ et $32 \%$, ce qui est inférieur aux taux observés, par exemple, en Norvège, où environ $27 \%$ des migrants pour raisons humanitaires occupent un emploi un an après leur arrivée et $43 \%$ trois ans après leur arrivée.

114. La situation générale des migrants sur le plan de l'emploi est donc contrastée. Celle des primoarrivants semble peu favorable comparativement à ce qui est observé dans d'autres pays, tandis que celle des migrants installés depuis plus longtemps paraît plutôt favorable. En l'absence de données longitudinales, d'une part, et de données se rapportant à des années différentes susceptibles de permettre des comparaisons, d'autre part, il est difficile de déterminer si ces résultats traduisent un processus de convergence tardif mais fort ou s'ils reflètent plutôt des effets de cohorte - à savoir que les migrants pour raisons humanitaires les plus récemment arrivés rencontrent davantage de difficultés à s'intégrer sur le marché du travail suisse. Le fait que ces migrants viennent essentiellement d'Afrique, tandis que ceux arrivés depuis plus longtemps sont souvent originaires d'Europe, semble plaider en faveur de la deuxième hypothèse, étant donné que les migrants originaires d'Europe s'intègrent en principe plus facilement sur le marché du travail dans tous les pays de l'OCDE.

\section{Programmes en faveur des immigrés pour raisons humanitaires}

115. Les immigrés arrivés dans le pays pour des raisons humanitaires forment le principal groupe visé par la politique d'intégration suisse, à tout le moins au niveau fédéral. Depuis 2008, l'État fédéral octroie aux cantons un forfait d'intégration de $6000 \mathrm{CHF}$ par réfugié reconnu ou personne admise à titre provisoire, affecté à «l'insertion professionnelle et à l'acquisition d'une langue nationale ». Pour $80 \%$, cette somme est versée à l'avance, tandis que les $20 \%$ restants sont octroyés après "intégration réussie ». L'ordonnance qui a instauré ce forfait dispose que l'intégration sera principalement mesurée sur la base du taux d'activité, «compte tenu de la situation sur le marché du travail dans le canton ». Toutefois, dans la pratique, dans la quasi-totalité des cas, le forfait d'intégration est versé sans qu'il soit tenu compte de cet " indicateur ». Les cantons utilisent ces fonds, d'une part pour apporter un soutien général aux programmes et mesures et, d'autre part, pour prendre en charge les frais de participation des bénéficiaires individuels. Le budget fédéral consacré au forfait d'intégration s'est élevé à 31 millions CHF en 2009 et à 56 millions CHF en 2010.

116. En outre, les autorités fédérales remboursent aux cantons les sommes payées au titre de l'aide sociale (versée à l'échelon infrafédéral) pendant les cinq années qui suivent l'entrée en Suisse, voire, pour les personnes admises à titre provisoire, pendant les sept premières années. Toutefois, les allocations d'aide sociale versées aux immigrés admis à titre provisoire sont nettement moins élevées que les prestations d'aide sociale normales. Dans la pratique, ce niveau moins élevé a souvent été imputé à une moindre volonté, de la part des cantons, d'intégrer ces migrants sur le marché du travail. Deux cantons, en l'occurrence les cantons de Lucerne et de Bâle-Ville, ont décidé de permettre aux personnes admises à titre provisoire d'accéder aux allocations d'aide sociale normales, et d'associer ces allocations à une aide intensive à l'intégration. Une évaluation de l'impact de cette mesure est actuellement en cours.

117. Au fond, la structure actuelle incite peu les cantons et les municipalités à favoriser l'intégration sur le marché du travail des réfugiés et des personnes admises à titre provisoire pendant les cinq à sept années qui suivent leur arrivée dans le pays. Il s'agit là d'une période cruciale, parce qu'elle a une forte valeur prédictive des résultats à long terme en matière d'intégration sur le marché du travail (OCDE, 2007). En réalité, l'offre de services structurés d'aide à l'intégration varie beaucoup selon les cantons et les municipalités. Les immigrés venus en Suisse pour des raisons humanitaires étant dispersés sur l'ensemble 
du territoire du pays, cette grande dispersion pose des problèmes du point de vue de l'accès à des services d'intégration et en matière d'égalité de traitement ${ }^{42}$.

118. Néanmoins, certaines municipalités et certains cantons ont mis en place des programmes pour faciliter l'intégration des migrants pour raisons humanitaires. En outre, l'Office fédéral des migrations finance des projets pilotes. Les programmes déjà en place sont principalement axés sur l'emploi peu qualifié dans les secteurs de l'hôtellerie et de la restauration, du commerce et de l'industrie, de la construction et des services de nettoyage (voir KEK-CDC, 2008). La plupart de ces programmes sont ouverts à l'ensemble des migrants pour raisons humanitaires et ne visent pas spécifiquement les primoarrivants. Il est difficile d'apprécier dans quelle mesure ils ont contribué aux taux d'emploi élevés observés actuellement, qui s'accompagnent d'une forte incidence de la surqualification parmi les migrants pour raisons humanitaires titulaires d'un diplôme de l'enseignement supérieur.

119. Contrairement à d'autres pays examinés par l'OCDE, en particulier aux pays nordiques, la plupart des cantons suisses ne sont pas dotés d'un programme d'intégration structuré ${ }^{43}$. Il ressort des données dont on dispose sur ce type de programmes - par exemple les données relatives au Danemark et à la Norvège - qu'ils peuvent, s'ils sont bien conçus, faciliter l'intégration des immigrés sur le marché du travail (voir OCDE, 2007, et Liebig, 2009). Étant donné la situation peu favorable sur le marché du travail des primo-arrivants qui sont venus en Suisse pour des raisons humanitaires, il est possible qu'un programme d'intégration structuré et spécialement conçu pour cette catégorie d'immigrés et leur famille ait des retombées positives non négligeables.

\section{L'emploi des femmes immigrées}

120. La Suisse est l'un des pays de l'OCDE qui affiche le taux d'emploi des femmes le plus élevé, y compris en ce qui concerne les femmes immigrées, avec un taux d'emploi global supérieur à $66 \%$ (voir tableau 7). L'un des problèmes que pose l'emploi des femmes en Suisse est la fréquence du travail à temps partiel. Ainsi, malgré un taux d'emploi féminin très élevé, le taux d'emploi des femmes en équivalents plein temps est moyen comparativement à celui d'autres pays. En Suisse, plus de la moitié des femmes qui travaillent occupent un emploi à temps partiel, contre un quart seulement en moyenne dans la zone OCDE (OCDE, 2006). La proportion de femmes immigrées travaillant à temps partiel est plus faible. Selon des données de l'ESPA relatives à 2009, la proportion de femmes employées à plein temps parmi les femmes d'âge actif s'établit à $31 \%$ pour les autochtones et à $32 \%$ pour les immigrées originaires de pays à faible revenu, alors que la proportion de femmes employées à temps partiel s'établit respectivement à $44 \%$ et $27 \%$. Par conséquent, globalement, la différence de taux d'emploi entre les femmes nées en Suisse et celles nées dans un pays à faible revenu s'explique par le fait que le travail à temps partiel est plus répandu parmi les premières.

121. L'existence de services de garde d'enfants influe de façon déterminante sur le taux d'activité des femmes; or, dans ce domaine, la Suisse accuse un retard par rapport à d'autres pays de l'OCDE (voir OCDE, 2009 et partie IV, infra). Les services d'éducation et d'accueil des jeunes enfants (ECEC) relèvent essentiellement de la compétence des cantons. Bien que l'offre de services dans ce domaine se soit nettement accrue ces vingt dernières années, cet accroissement a surtout concerné les cantons qui étaient déjà relativement bien dotés (voir Office fédéral de la statistique, 2008). Il s'agit plus précisément des cantons urbains de Genève, Zurich et Bâle. En 2005, une vaste étude sur les services ECEC a révélé que

\footnotetext{
42 À l'évidence, cette disparité de l'offre de services relevant des cantons touche également les autochtones. Toutefois, la situation des migrants pour raisons humanitaires - notamment de ceux admis provisoirement - est particulière, puisqu'ils ne jouissent pas pleinement du droit de libre circulation sur le territoire suisse et ne sont pas libres de choisir leur canton de résidence. 
dans l'ensemble, l'offre existante ne couvrait que $40 \%$ des besoins potentiels de la population suisse (Swiss National Fund, 2005). L'étude indiquait aussi qu'à caractéristiques observables comparables, les familles originaires de l'ex-Yougoslavie font plus appel aux services ECEC que les familles autochtones. Cette tendance n'a toutefois pas été observée parmi les autres catégories de migrants ${ }^{44}$.

122. Le tableau 7 donne une image plus précise des déterminants de l'emploi des femmes immigrées en Suisse. Plusieurs tendances importantes s'en dégagent. Premièrement, il existe un écart de taux d'emploi important entre les différentes catégories d'immigrées, les femmes originaires de Turquie affichant le taux d'emploi le plus faible. Deuxièmement, le handicap des femmes originaires de Turquie et de l'ex-Yougoslavie sur le marché du travail s'explique au moins pour moitié par le fait que la structure d'âge et de formation de ces groupes est moins favorable que celle des autochtones. Troisièmement, le fait d'avoir un enfant de moins de six ans a un impact particulièrement négatif sur le taux d'emploi des immigrées originaires de Turquie et de pays à faible revenu autres que l'ex-Yougoslavie. Il est possible que le recours important des femmes originaires de l'ex-Yougoslavie aux services d'ECEC explique pour partie le moindre impact de leur condition de parent sur leur taux d'emploi.

123. Le tableau 7 présente également les liens entre la présence d'enfants dans le ménage et l'emploi pour les femmes immigrées comparativement aux femmes autochtones, en distinguant travail à temps plein et travail à temps partiel. C'est pour le travail à temps partiel que l'écart est le plus grand. Toutefois, comme le suggère la corrélation positive pour les immigrées originaires des pays à faible niveau de revenus dans le modèle $4 \mathrm{a}$, le fait d'avoir des enfants est associé à une diminution nettement plus forte de la probabilité de travailler à temps plein parmi les femmes autochtones que parmi les immigrées originaires de pays à faible revenu.

Tableau 7 : Déterminants de l'emploi des femmes immigrées en Suisse, 15-64 ans, 2009

\begin{tabular}{|c|c|c|c|c|c|c|c|c|}
\hline Variables & (1) & (2) & (3) & $\begin{array}{c}\text { (3a) emploi } \\
\text { à plein } \\
\text { temps / sans } \\
\text { emploi }\end{array}$ & $\begin{array}{c}\text { (3b) emploi } \\
\text { à temps } \\
\text { partiel / sans } \\
\text { emploi }\end{array}$ & (4) & $\begin{array}{c}\text { (4a) emploi } \\
\text { à plein } \\
\text { temps / sans } \\
\text { emploi }\end{array}$ & $\begin{array}{c}\text { (4b) emploi } \\
\text { à temps } \\
\text { partiel / sans } \\
\text { emploi }\end{array}$ \\
\hline Turquie & $-24^{* * *}$ & $-12^{\star \star \star}$ & $-10^{\star \star *}$ & -1 & $-14^{\star \star *}$ & $-11^{* *}$ & $-9^{*}$ & $-14^{\star \star *}$ \\
\hline ex-Yougoslavie & $-14^{* * *}$ & $-6^{* * *}$ & $-5^{* * *}$ & $6^{* * *}$ & $-12^{\star * *}$ & $-8^{* * *}$ & -2 & $-16^{\star * *}$ \\
\hline autres pays à bas revenu & $-16^{\star * *}$ & $-16^{\star \star \star}$ & $-15^{\star \star *}$ & $-8^{\star \star \star}$ & $-22^{* * *}$ & $-15^{\star \star *}$ & $-16^{* * *}$ & $-22^{\star \star *}$ \\
\hline pays à revenu élevé & $-2^{*}$ & -1 & 0 & $6^{\star \star \star}$ & $-5^{\star \star \star}$ & 0 & $3^{*}$ & $-5^{\star * *}$ \\
\hline avec au moins un enfant de moins de 6 ans & & & $-11^{\star * *}$ & $-35^{\star * *}$ & 0 & $-9^{* * *}$ & $-40^{\star * *}$ & 2 \\
\hline avec au moins un enfant de 6 à 17 ans & & & 0 & $-13^{\text {***}}$ & $10^{* \star *}$ & 0 & $-18^{* * *}$ & $9^{* * *}$ \\
\hline enfant de moins de 6 ans * né en Turquie & & & & & & $-17^{* *}$ & 5 & $-17^{* *}$ \\
\hline enfant de 6 à 17 ans * né en Turquie & & & & & & $11^{*}$ & $19^{\star \star *}$ & 9 \\
\hline enfant de moins de 6 ans * né en ex-Yougoslavie & & & & & & -5 & $9^{* *}$ & 0 \\
\hline enfant de 6 à 17 ans * né en ex-Yougoslavie & & & & & & $8^{* \star *}$ & $18^{* * *}$ & $7^{*}$ \\
\hline $\begin{array}{l}\text { enfant de moins de } 6 \text { ans * né dans un pays à } \\
\text { bas revenu }\end{array}$ & & & & & & $-9^{* *}$ & $13^{\star \star *}$ & $-10^{* *}$ \\
\hline $\begin{array}{l}\text { enfant de } 6 \text { à } 17 \text { ans * né dans un pays à bas } \\
\text { revenu }\end{array}$ & & & & & & $6^{* *}$ & $18^{\star \star \star}$ & 6 \\
\hline $\begin{array}{l}\text { enfant de moins de } 6 \text { ans * né dans un pays à } \\
\text { revenu élevé }\end{array}$ & & & & & & -2 & $10^{\star \star \star}$ & -3 \\
\hline $\begin{array}{l}\text { enfant de } 6 \text { à } 17 \text { ans * né dans un pays à revenu } \\
\text { élevé }\end{array}$ & & & & & & 0 & $9^{* * *}$ & 0 \\
\hline Nombre d'observations & 19616 & 19578 & 19578 & 12026 & 13266 & 19578 & 12026 & 13266 \\
\hline
\end{tabular}

Note : La variable dépendante est la variable dichotomique "employé". Les coefficients des variables indépendantes sont basés sur une régression des MCO des taux d'emploi des femmes de 15-64 ans. Tous les modèles incluent une constante. Les modèles 2-6 incluent une variable de contrôle pour l'âge et le niveau d'éducation atteint. * ${ }^{\star *},{ }^{* \star *}$ indiquent une significativité aux seuils de $1 \%$, $5 \%$ et $10 \%$. "Né en", "Né dans", réfèrent au pays de naissance de la femme. Le groupe de référence est constitué des femmes nées dans le pays. Dans les colonnes $3 \mathrm{a} / \mathrm{b}$ et $4 \mathrm{a} / \mathrm{b}$, un coefficient positif est associé à une plus grande probabilité d'être employé à temps plein/partiel, le groupe de référence étant les personnes sans emploi.

Source : Enquête suisse sur la population active, Office fédéral de la statistique, Suisse.

44 Malheureusement, l'étude ne prenait en compte que les familles immigrées originaires de l'ex-Yougoslavie, du Portugal et d'Italie. 
124. Il existe peu de mesures destinées à favoriser l'intégration des immigrées. La preuve en est que, d'après les données de l'ESPA, seulement $15 \%$ des immigrées originaires de pays non limitrophes de la Suisse (c'est-à-dire qui n'ont pas de langue commune avec la Suisse) ont suivi des cours de langue.

125. En réalité, pour beaucoup d'immigrées, il est difficile d'accéder aux services proposés à la population en général. Les mesures mises en place par les services cantonaux de l'emploi s'adressent principalement aux bénéficiaires de l'assurance chômage, c'est-à-dire à des personnes qui ont déjà travaillé en Suisse. Depuis 2011, les services cantonaux de l'emploi pouvaient toutefois étendre le bénéfice de leurs prestations à d'autres groupes, mais cette disposition a été limitée dans le cadre de la récente révision de la loi relative à l'assurance chômage.

\section{Discrimination sur le marché du travail}

126. La question de la discrimination à l'encontre des migrants a jusqu'à présent suscité peu d'intérêt en Suisse. Il n'existe ni législation visant spécifiquement cette question, ni institution chargée de porter les affaires de discrimination devant les tribunaux (voir OCDE, 2008c ${ }^{45}$. Le cadre qui régit la lutte contre la discrimination est donc moins développé que dans les autres pays européens membres de l'OCDE ${ }^{46}$.

127. À l'échelon fédéral, la Commission fédérale contre le racisme apporte des conseils aux victimes de discrimination, mais n'est pas habilitée à saisir la justice ou à appliquer des sanctions à l'encontre des employeurs. À l'échelon infrafédéral, il existe des «centres de consultation » chargés de donner des conseils en cas de discrimination par des personnes privées. Sept de ces centres (dont la Commission fédérale contre le racisme) ont uni leurs forces en constituant un réseau. En 2010, ce réseau n'a traité que 23 affaires de discrimination sur le marché du travail. Le nombre limité d'affaires est peut-être lié au fait que le cadre juridique existant n'est pas clair au sujet des sanctions applicables en cas de discrimination à l'embauche ${ }^{47}$.

128. S'agissant de la discrimination par les autorités ou les institutions publiques, il existe, au sein du Département fédéral de l'intérieur, un Service de lutte contre le racisme (SLR). Contrairement à la Commission fédérale contre le racisme, le SLR dispose d'un petit budget - environ 1 million CHF - pour financer des projets destinés à lutter contre la discrimination au sein de l'administration fédérale. En outre, à l'échelon cantonal et municipal, une douzaine de bureaux de médiation proposent des conseils en matière de discrimination.

129. L'absence de cadre institutionnel fort en matière de lutte contre la discrimination est particulièrement flagrante dans le cas de la discrimination sur le marché du travail, où la liberté contractuelle l'emporte et où rien n'empêche a priori les employeurs de recruter sur la base de critères comme l'origine ${ }^{48}$. Cette situation, outre qu'historiquement les immigrés n'ont eu qu'assez peu de

45 Toutefois, certaines dispositions légales permettent en principe aux travailleurs victimes de discrimination de saisir la justice. En outre, la Commission fédérale contre le racisme et le Service de lutte contre le racisme peuvent conseiller et accompagner les victimes de discrimination. De plus, dans certains cantons, des institutions peuvent également apporter une aide. Enfin, depuis 2002, les ressortissants des pays de l'UE sont protégés contre les discriminations sur le marché du travail grâce aux dispositions de l'accord sur la libre circulation passé entre la Suisse et les pays de l'UE.

Tous les pays de l'OCDE membres de l'Union européenne sont dotés d'un cadre élémentaire de lutte contre les discriminations ethniques, qui repose sur la directive anti-discrimination adoptée par l'Union européenne ; pour la Norvège, voir Liebig (2009). Pour un tour d'horizon de la législation anti-discrimination en général, voir OCDE (2008).

$47 \quad$ Pour une analyse critique approfondie du système actuel, voir CFR (2009).

48

À condition qu'ils n'expriment pas leurs préférences, ce qui pourrait constituer une discrimination publique au sens de l'article 261 bis du Code pénal. La discrimination sexuelle est réprimée par une loi spécifique (la loi sur l'égalité). Voir à ce propos Fibbi (2005). 
difficultés à entrer sur le marché du travail suisse, font que la question de la discrimination occupe peu de place dans le débat public et intéresse peu les médias. Aujourd'hui encore, beaucoup d'acteurs de la société suisse ne sont pas convaincus qu'il existe une discrimination systématique sur le marché du travail, et les politiques destinées à lutter contre la discrimination sur le marché du travail sont peu nombreuses ${ }^{49}$.

130. Comme indiqué précédemment, les immigrés originaires de pays à faible revenu sont désavantagés sur le marché du travail suisse, même après prise en compte de diverses caractéristiques sociodémographiques et d'un indicateur objectif des compétences. Les différences au niveau des résultats en matière d'emploi constatées après neutralisation des effets de ces caractéristiques sont souvent interprétées comme un indicateur indirect de discrimination (voir de Coulon et Flückiger, 2000). Toutefois, il est toujours possible qu'elles s'expliquent par d'autres facteurs, qui n'ont pas été explicitement pris en compte ou ne sont pas observables directement.

131. Des tests expérimentaux à grande échelle sur les procédures de recrutement conduits dans plusieurs pays de l'OCDE, ces dernières années, permettent de surmonter les difficultés à démontrer l'existence de discrimination. Ces tests mettent en évidence un comportement fortement discriminatoire des employeurs (voir Simeone, 2005). Ils consistent à soumettre, pour un même poste, les candidatures de deux candidats (fictifs) se distinguant principalement par leur nom. Les deux candidats devant avoir sensiblement la même qualification, le test porte essentiellement sur des personnes qui ont obtenu leur diplôme le plus élevé dans le pays d'accueil, c'est-à-dire des immigrés de la deuxième génération. Un nombre suffisant de candidatures doit être envoyé pour exclure l'effet du hasard. Si les candidatures des candidats d'une origine donnée sont rejetées plus souvent que celles des autres, on en déduit qu'il y a discrimination. Ces études ont mis en évidence l'existence d'une forte discrimination à l'embauche dans six des neufs pays étudiés à ce jour (Belgique, Danemark, France, Allemagne, Pays-Bas et Suède).

132. Une étude de ce type a été conduite en Suisse, en 2002, pour le compte du Fonds national suisse de la recherche scientifique (Fibbi et al., 2004), sur la base de candidatures fictives déposées par des hommes jeunes à la recherche de leur premier emploi fixe et titulaires de diplômes décernés par des établissements d'enseignement suisses. Quatre groupes d'immigrés ont été étudiés - originaires du Portugal en Suisse romande, de Turquie en Suisse alémanique et originaires de l'ex-Yougoslavie dans ces deux parties du pays ${ }^{50}$. L'étude prévoyait que les candidats convoqués à un entretien d'embauche envoient un courrier de refus à leur convocation. Souvent, les candidats immigrés n'étaient convoqués qu'après envoi de la lettre de refus par le candidat autochtone. Il n'est pas aisé de déterminer si cette différence de traitement doit être analysée comme une discrimination dans la mesure où il n'est pas possible d'établir un lien de causalité entre le décalage dans l'envoi de la convocation aux deux candidats et le refus de la proposition par le premier candidat. Le tableau 8 présente une synthèse des résultats de l'étude. Il montre que la discrimination à l'égard des immigrés dans le processus de recrutement semble un phénomène courant en Suisse. Un candidat d'origine turque doit déposer trois fois plus de candidatures qu'un Suisse présentant, par ailleurs, les mêmes caractéristiques pour être convoqué à un entretien d'embauche et les candidats originaires de l'ex-Yougoslavie doivent en déposer jusqu'à cinq fois plus ${ }^{51}$. La différence non

49 Les considérants d'un arrêt rendu en 2002 par le Tribunal fédéral sont peut-être symptomatiques de cette situation. En l'espèce, le tribunal a rejeté à l'unanimité la requête formulée par deux jeunes filles - vivant avec leur mère suisse, divorcée de leur père albanais - pour obtenir l'autorisation de porter le nom de leur mère. Le tribunal a contesté le fait qu'un nom albanais puisse être préjudiciable à leur avenir professionnel. Il n'a pas exclu la possibilité qu'un nom à consonance balkanique puisse parfois jouer en défaveur des postulants à un emploi, mais a estimé que « dans la grande majorité des cas, pareille influence n'est ni démontrée, ni même suspectée » (arrêt 5C.163/2002 du 01.10.02).

Le lieu de naissance indiqué dans les candidatures fictives était le Kosovo et la « langue maternelle » était l'albanais.

51 En réalité, cette limite supérieure est peut-être nettement plus élevée dans la mesure où l'étude ne tenait pas compte des offres d'emploi exigeant des candidats de nationalité suisse ou de langue maternelle suisse-allemande. 
négligeable entre les deux indicateurs possibles porte à croire que les tensions qui règnent sur le marché du travail suisse limitent peut-être la discrimination.

133. Plus généralement, il semble que le groupe le plus important numériquement - les immigrés originaires de l'ex-Yougoslavie - soit aussi celui qui est le plus visé par la discrimination. La discrimination semble aussi plus forte en Suisse alémanique. L'étude révèle également que l'ampleur de la discrimination ne dépend pas de la taille de l'entreprise ou du secteur d'activité, mais que la tendance à la discrimination est légèrement plus marquée pour les emplois qui supposent des contacts avec la clientèle.

Tableau 8: Nombre estimé de candidatures envoyées par les différents groupes d'immigrés afin de recevoir une convocation à un entretien d'embauche, par rapport à un candidat suisse ayant les mêmes caractéristiques

\begin{tabular}{lcc}
\hline & Limite maximale & Limite minimale \\
\hline Portugais dans la partie francophone & 2.1 & aucune différence \\
ex-Yougoslaves dans la partie francophone & 2.9 & 1.3 \\
Turcs dans la partie alémanique & 3.3 & 1.4 \\
ex-Yougoslaves dans la partie alémanique & 4.8 & 2.5 \\
\hline
\end{tabular}

Source : Calculs de l'OCDE basés sur les données de Fibbi, Kaya et Piguet (2002).

134. Dans l'ensemble, il est permis de penser que le grand public et les employeurs confondent la préférence - inscrite dans la loi - accordée à la population résidente, suisse et étrangère, en matière d'immigration (une personne ne peut être admise dans le pays pour y travailler que si aucun Suisse, ressortissant de l'Union européenne ou étranger déjà installé ne peut occuper le poste) et l'attitude à avoir vis-à-vis des étrangers à la recherche d'un emploi qui résident légalement en Suisse et bénéficient, selon la loi, d'une égalité d'accès au marché du travail. Une enquête nationale régulière réalisée en 2001 comportait la question suivante : «Estimez-vous que les citoyens suisses devraient bénéficier d'une priorité sur le marché du travail ? $»^{52}$. Elle a démontré qu'une majorité de Suisses étaient favorables à la préférence nationale à l'embauche (33\% entièrement favorables et $27 \%$ partiellement favorables) et que $12 \%$ seulement de la population y étaient fortement opposés (Raymann, 2003).

135. De fait, il n'est pas rare que la «nationalité suisse » soit exigée dans les offres d'emploi des entreprises privées ${ }^{53}$. Contrairement à ce qui est observé dans d'autres pays de l'OCDE (voir, par exemple, OCDE, 2008b et OCDE, 2008c), en général, cette forme de discrimination n'est pour l'instant pas illégale en Suisse ${ }^{54}$.

136. Il semble que le renforcement du cadre anti-discrimination en Suisse ne fasse pas de doute. Toutefois, il ne faut pas fonder trop d'espoirs sur des mesures strictement juridiques, même assorties d'importants moyens d'application et de fortes pénalités. Même dans les pays de l'OCDE dotés d'un cadre fort et bien établi en matière de lutte contre les discriminations, ces mesures ne permettent de s'attaquer qu'à la partie émergée de l'iceberg. Pour mettre fin à la discrimination à l'embauche, il est important que le problème soit mis au premier plan. D'autres pays de l'OCDE, allant au-delà de la mise en place d'un cadre juridique de lutte contre la discrimination, adoptent des «politiques en faveur de la diversité » (encadré 3).

\footnotetext{
$52 \quad$ Cette enquête n'a pas été répétée depuis lors.

53 En Suisse alémanique, la « langue maternelle suisse-allemande » est également parfois exigée.

54 Toutefois, les ressortissants de l'UE sont quelque peu protégés de cette forme de discrimination parce qu'ils peuvent invoquer l'accord sur la libre circulation.
} 


\section{Encadré 3. Politiques visant à promouvoir la diversité}

Contrairement à la plupart des autres pays de l'OCDE, la Suisse ne dispose pas d'une législation complète contre la discrimination pour remédier à la discrimination à l'embauche. En outre, même dans les pays qui disposent d'un système avancé dans ce domaine, la discrimination s'est révélée difficile à détecter ou à prouver. Dans tous les pays, le nombre de plaintes liées à la discrimination à l'embauche est réduit par rapport à l'ampleur de la discrimination mise en lumière par les tests en situation réalisés dans de nombreux pays de l'OCDE, dont la Suisse. Le manque d'efficacité de la législation anti-discrimination et la persistance d'autres obstacles structurels à l'emploi des immigrés et de leurs enfants ont incité les pouvoirs publics à prendre des mesures plus volontaristes. Une nouvelle stratégie, connue sous le nom de politique de la diversité, prend forme ces dernières années dans nombre de pays de l'OCDE. S'inspirant de politiques néerlandaises datant des années 90, la Belgique, en particulier, est récemment devenue un pays pionnier en la matière. Les politiques en faveur de la diversité visent à garantir aux groupes défavorisés (dont les immigrés et leurs enfants) l'égalité des chances sur le marché du travail au moyen d'incitations et de mesures fortement ciblées, quoique de manière indirecte. À titre d'exemple, la Belgique (notamment la Flandre), réserve, pendant une période limitée, l'accès à certains postes aux groupes défavorisés et offre un soutien financier et administratif aux entreprises qui s'efforcent de veiller à la diversité du personnel dans les processus de recrutement et d'avancement. Les premiers résultats d'une évaluation de cette politique indiquent que celle-ci semble avoir contribué aux améliorations récemment constatées en matière d'intégration sur le marché du travail, notamment pour les enfants d'immigrés (voir Van der Voorde et de Bruijn, 2010).

Les initiatives lancées dans d'autres pays de l'OCDE ont été moins ambitieuses mais sont également de plus en plus nombreuses (voir OCDE, 2008b). En France, par exemple, les entreprises ont la possibilité de se soumettre à un audit destiné à déterminer si leurs pratiques en matière de recrutement et de promotion sont discriminatoires. Si elles obtiennent de bons résultats à l'audit et si elles ont mis en œuvre des mesures supplémentaires pour promouvoir la diversité, elles peuvent obtenir un «label diversité » décerné par les autorités compétentes en matière d'intégration. Pour recevoir ce label, elles doivent satisfaire à six critères : un engagement formel en faveur de la diversité ; un rôle actif des partenaires sociaux au sein de l'entreprise ; des procédures de gestion des ressources humaines équitables ; une communication sur la diversité ; la mise en œuvre d'actions citoyennes concrètes en faveur de la diversité ; des procédures d'évaluation des pratiques. La France, comme de plus en plus d'autres pays de l'OCDE, notamment la Belgique, les Pays-Bas et l'Allemagne, œuvre aussi en faveur d'une «charte de la diversité » dont les signataires s'engagent à favoriser le pluralisme à travers le recrutement et la gestion des carrières, dans le cadre d'une stratégie axée sur le progrès de l'entreprise, sur son efficacité et sur la qualité de ses relations sociales. En l'absence de suivi précis des recrutements et de l'évolution des carrières au sein des entreprises signataires, il est difficile d'avoir une vision précise de l'efficacité de ce type de mesure. II se produit sans nul doute un phénomène d'autosélection des entreprises déjà engagées en faveur de la diversité parmi les signataires bien qu'il ne soit probablement pas inutile de donner un caractère formel à la procédure pour diffuser les normes dans l'ensemble de l'entreprise. Plusieurs pays de l'OCDE, notamment la France, l'Allemagne et la Norvège, ont également testé les CV anonymes, bien que l'évidence de leur efficacité n'ait pas été démontrée.

En Belgique, en Norvège et aux Pays-Bas, on a observé un réel effort pour améliorer la diversité dans le secteur public. Les politiques en place sont axées sur les différents aspects du processus de recrutement pour lesquels les immigrés et leurs enfants sont structurellement défavorisés. Parmi ces politiques figurent l'introduction à grande échelle du CV anonyme, la promotion ciblée de l'apprentissage pour les jeunes issus de l'immigration, l'offre de stages de nature à leur permettre d'entrer dans la vie active et la mise en place d'une formation spéciale pour les aider à réussir les tests de recrutement.

En Suisse, aucun outil de promotion de la diversité de ce type n'a été introduit jusqu'à présent hormis des projets à petite échelle mis en œuvre au niveau cantonal et quelques mesures volontaires prises par des employeurs (voir Schönenberger et Piguet, 2010).

137. Globalement, d'importants progrès peuvent et doivent être accomplis dans le domaine de la discrimination, sur le plan du cadre législatif et institutionnel et sur celui des mesures de sensibilisation à cette problématique et de lutte contre la discrimination sur le marché du travail.

\section{La naturalisation et son impact}

138. L'accès à la nationalité du pays d'accueil est un instrument important de la politique d'intégration. L'OCDE a montré en 2011 que la naturalisation influe généralement positivement sur les résultats des immigrés en matière d'emploi à travers différents canaux (2011 b). Parmi ces canaux figure la suppression des obstacles institutionnels à l'accès au marché du travail, en particulier à l'accès aux emplois 
publics et qualifiés. En outre, la naturalisation semble être perçue par les employeurs comme le signe d'un meilleur « potentiel d'intégration », qui peut aller de pair avec une productivité plus élevée (en raison, par exemple, d'une meilleure maitrise de la langue ou d'une plus forte motivation). À titre d'exemple, des études fondées sur des tests en situation ont montré qu'à caractéristiques équivalentes, les immigrés naturalisés sont plus souvent convoqués à un entretien d'embauche que des candidats immigrés non naturalisés. Cette fonction de signal de la naturalisation est plus ou moins forte selon qu'il est, ou non, usuel d'indiquer sa nationalité dans le processus de candidature (si cette mention n'est pas exigée pour l'emploi lui-même, ce qui est rare). En Suisse, il est courant d'indiquer sa nationalité dans une candidature, ce qui porte à croire que cette fonction de signal joue peut-être un rôle très important.

139. Les critères auxquels est subordonné l'accès à la citoyenneté sont très variables d'un pays de l'OCDE à l'autre et le système de naturalisation de la Suisse est, à bien des égards, l'un des plus restrictifs de l'OCDE, sachant toutefois qu'il est supposé en Suisse couronner un processus d'intégration réussie. Par exemple, la durée minimale de résidence exigée dans le cadre de la procédure de naturalisation ordinaire est de douze ans, ce qui constitue la durée la plus longue de l'OCDE. D'autre part, le système de naturalisation suisse se distingue par le fait que la citoyenneté suisse comporte trois niveaux (voir encadré 4). Il s'ensuit qu'un peu plus d'un tiers seulement des immigrés résidant dans le pays depuis plus de dix ans ont la nationalité suisse, contre plus de $60 \%$, en moyenne, dans les pays de l'OCDE. Le Luxembourg est le seul pays où le pourcentage d'immigrés résidents de longue durée non naturalisés est plus élevé.

140. Cette politique de naturalisation restrictive, conjuguée à la longue tradition d'immigration de la Suisse, se traduit par le fait que plus de $6 \%$ de la population née dans le pays n'ont pas de passeport suisse. En réalité, la Suisse est, des pays de l'OCDE pour lesquels des données comparables sur la nationalité des immigrés de la deuxième génération sont disponibles, celui qui affiche le plus faible pourcentage de personnes naturalisées parmi cette population (graphique 13) ${ }^{55}$.

Graphique 13: Pourcentage des enfants d'immigrés venant des pays à bas revenus, nés dans le pays, âgés de 20-29 ans et non scolarisés, autour de 2007

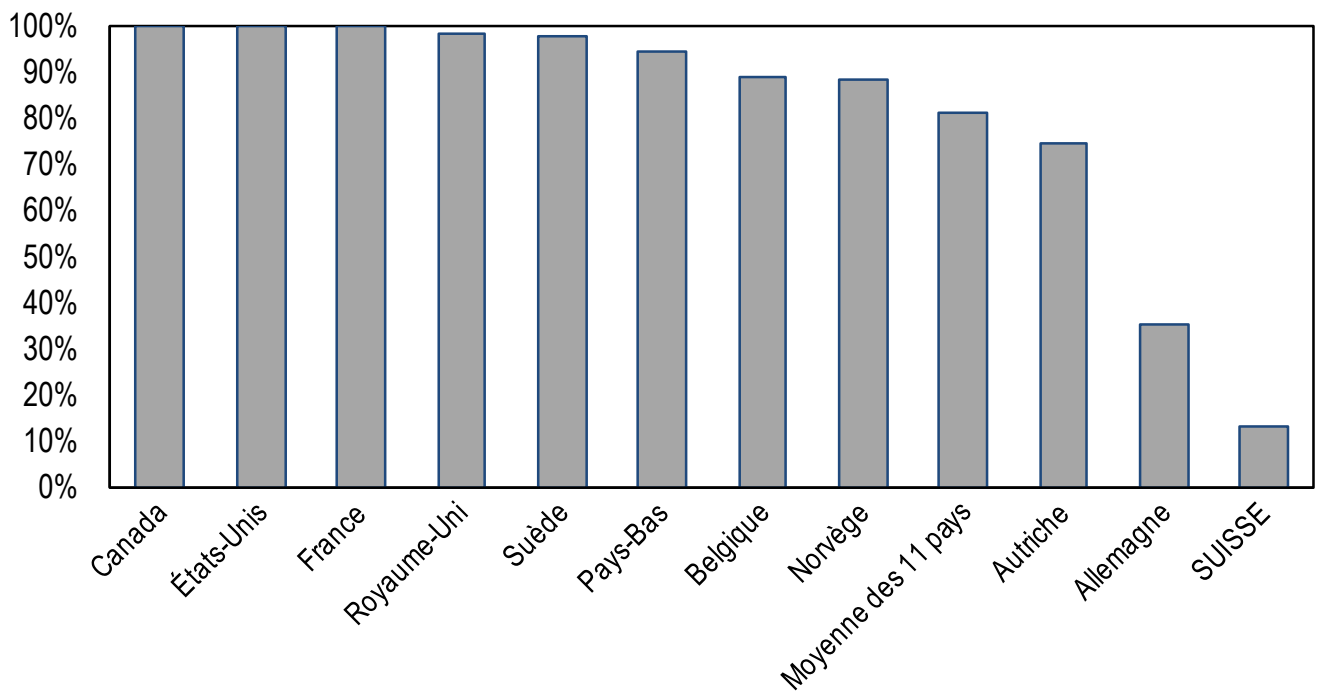

Source : Voir Liebig et Von Haaren (2011).

55 Cependant, certaines facilités existent pour les enfants d'immigrés, en particulier, dans le calcul de la durée des conditions de résidence, les années de résidence en Suisse entre 10 et 20 ans sont comptées deux fois. 
DELSA/ELSA/WD/SEM(2012)2

\section{Encadré 4 : Accès à la nationalité suisse}

La Suisse est l'un des pays de l'OCDE où les conditions de naturalisation imposées aux étrangers sont particulièrement sévères. Pour la procédure ordinaire, la durée minimale de résidence exigée est de 12 ans, ce qui constitue une durée beaucoup plus longue que celle exigée dans les autres pays de l'OCDE, où elle est généralement comprise entre cinq et huit ans (voir Guimezanes, 2011). De surcroît, la Suisse applique le principe du droit du sang (ius sanguinis), aux termes duquel les immigrés de la deuxième génération n'acquièrent pas automatiquement la nationalité suisse.

La nationalité suisse se singularise par le fait qu'elle comporte trois niveaux. Avoir la citoyenneté suisse suppose d'avoir aussi la citoyenneté cantonale et communale et ces trois échelons administratifs imposent leurs propres conditions de naturalisation. En conséquence, l'administration fédérale ne peut pas faire appliquer une décision de naturalisation si les autorités cantonales ou municipales y sont défavorables, même si les conditions officielles sont remplies. Les autorités infrafédérales exigent généralement une durée minimale de résidence, pouvant aller jusqu'à dix ans, dans le canton et la municipalité concernés. Ces règles tendent à entraver la mobilité professionnelle et géographique.

Autrefois, certaines municipalités soumettaient la naturalisation à un vote populaire. Le Tribunal fédéral a toutefois mis fin à cette pratique répandue en 2003, en arrêtant que la naturalisation constituait un acte administratif. Les refus de naturalisation doivent donc être motivés, ce qui n'est pas possible en cas de vote populaire.

Plusieurs tentatives, dont la dernière remonte à 2004, ont été effectuées pour libéraliser l'accès à la citoyenneté suisse, en particulier pour les immigrés de la deuxième génération qui ont été élevés et scolarisés en Suisse. Bien que ces tentatives de réforme de fond aient échoué, certaines modifications importantes des règles d'accès à la citoyenneté suisse ont été apportées depuis 2004 et ont libéralisé cet accès. La principale d'entre elles est la réforme introduite en 2006, qui interdit aux cantons et aux municipalités d'exiger des émoluments de naturalisation supérieurs aux frais de procédure. Auparavant, ces émoluments pouvaient, dans certaines municipalités, s'élever à plusieurs milliers de francs suisses.

Une réforme de fond de la législation sur la citoyenneté suisse est actuellement en préparation. II est notamment envisagé de ramener de douze à huit ans la durée de résidence exigée dans le cadre de la procédure ordinaire et d'imposer des limites à la durée de résidence exigée par les autorités municipales et cantonales. Si cette réforme est votée, l'accès à la nationalité restera plus difficile en Suisse que dans la plupart des autres pays de l'OCDE, mais les pratiques de la Suisse se rapprocheront de celles des autres pays.

141. Dans toute analyse, il ne faut jamais oublier que les immigrés naturalisés et ceux qui ne le sont pas diffèrent à maints égards parce que la naturalisation est un processus de sélection. Les candidats à la naturalisation doivent déposer une demande et satisfaire à un certain nombre de critères. Pour étudier l'impact de la naturalisation, il faut disposer de données longitudinales permettant de comparer les immigrés au fil du temps. Or aucune étude longitudinale de ce type n'a été réalisée en Suisse à ce jour ${ }^{56}$. Steinhardt et al. (2010) utilisent des données transversales de l'ESPA pour comparer les résultats en matière d'emploi d'immigrés naturalisés et d'immigrés non naturalisés. Il en ressort, même après neutralisation des effets de diverses caractéristiques sociodémographiques, que le taux d'emploi et les salaires sont plus élevés parmi les immigrés naturalisés que parmi les autres, même si la situation de l'emploi des immigrés naturalisés reste moins favorable que celle des autochtones.

142. Liebig et Von Haaren (2011) s'appuient eux aussi sur des données transversales pour comparer les résultats en matière d'emploi des immigrés naturalisés et non naturalisés dans 14 pays de l'OCDE. En Suisse, les immigrés naturalisés de sexe masculin ont une probabilité d'occuper un emploi qualifié supérieure de presque $10 \%$ à celle d'immigrés non naturalisés présentant, par ailleurs, des caractéristiques

56 Des études longitudinales portant sur d'autres pays de l'OCDE ont toutefois été réalisées. Il en ressort que la naturalisation tend à avoir un effet positif sur les résultats en matière d'emploi, en particulier pour les immigrés les plus défavorisés. Toutefois, l'ampleur de ces retombées est très variable selon le pays et le groupe de migrants (voir OCDE, 2011, pour une analyse exhaustive de cette question). 
observables comparables (voir tableau 9). L'écart est particulièrement grand pour les hommes immigrés. En outre, une comparaison avec d'autres pays de l'OCDE confirme aussi ce résultat. L'accès à des postes plus qualifiés est en effet plus fortement corrélé à la naturalisation en Suisse qu'ailleurs, ce qui peut en partie s'expliquer par le fait que, dans ce pays, les conditions de naturalisation entravent la mobilité géographique des étrangers (voir également l'encadré 4).

\section{Tableau 9 : Estimation de la différence de probabilité d'occuper un poste hautement qualifié entre un naturalisé et un non-naturalisé, autour de 2007, 15-64 ans}

\begin{tabular}{|c|c|c|c|c|c|c|}
\hline & \multicolumn{2}{|c|}{$\begin{array}{l}\text { Ensemble des } \\
\text { immigrés }\end{array}$} & \multicolumn{2}{|c|}{$\begin{array}{l}\text { Immigrés nés dans un } \\
\text { pays à revenus élevés }\end{array}$} & \multicolumn{2}{|c|}{$\begin{array}{c}\text { Immigrés nés dans un } \\
\text { pays à bas revenus }\end{array}$} \\
\hline & Hommes & Femmes & Hommes & Femmes & Hommes & Femmes \\
\hline Autriche & $5^{\star \star \star}$ & $(-1)$ & $9^{* \star *}$ & $-11^{\star \star *}$ & $5^{\star \star \star}$ & $4^{\star \star}$ \\
\hline Belgique & $(-4)$ & $(-1)$ & $(-4)$ & $(-2)$ & $(1)$ & $8^{* *}$ \\
\hline Canada & $1^{* \star *}$ & $2^{\star * \star}$ & & & & \\
\hline Suisse & $9^{\star \star \star}$ & $5^{\star \star}$ & $8^{\star \star}$ & (2) & $10^{\star \star \star}$ & $7^{\star \star}$ \\
\hline Allemagne & $-2^{\star \star \star}$ & $3^{* *}$ & $-8^{\star \star \star}$ & $4^{* *}$ & $3^{\star \star \star}$ & $4^{\star \star \star}$ \\
\hline Danemark & $12^{\star \star \star}$ & $8^{* *}$ & $13^{\star \star \star}$ & (0) & $12^{\star \star \star}$ & $12^{\star \star \star}$ \\
\hline Espagne & $6^{*}$ & (0) & (2) & $(-5)$ & $11^{\star * \star}$ & $(4)$ \\
\hline France & $7^{\star \star *}$ & $3^{* *}$ & $10^{\star \star \star}$ & (3) & $5^{\star * *}$ & (3) \\
\hline Luxembourg & (1) & $(-4)$ & $(0)$ & $(-6)$ & (7) & (1) \\
\hline Pays-Bas & (0) & $(-2)$ & $(-2)$ & $(-2)$ & $5^{\star \star}$ & (1) \\
\hline Norvège & (5) & $-12^{* * *}$ & (4) & $(-8)$ & $17^{* *}$ & $-19^{* *}$ \\
\hline Suède & $(-1)$ & $(1)$ & (2) & (5) & $11^{\star \star \star}$ & $(-1)$ \\
\hline Royaume-Uni & (2) & (2) & (3) & (2) & $5^{\star *}$ & (2) \\
\hline États-Unis & (2) & $5^{* * *}$ & (1) & (4) & $2^{*}$ & $5^{\star * *}$ \\
\hline $\begin{array}{l}\text { États-Unis } \\
\text { (Excl.irrég). }\end{array}$ & (1) & $4^{\star \star \star}$ & (1) & (4) & -1 & $4^{* *}$ \\
\hline
\end{tabular}

Note : L'échantillon est limité aux personnes employées. Le tableau montre les coefficients attachés à la naturalisation en points de pourcentage. La variable dépendante est la variable dichotomique "Employé dans une profession hautement qualifiée". La régression inclut les variables de contrôle pays de naissance, âge et éducation.

Source et notes approfondies : Voir Liebig et Von Haaren (2011).

143. En Suisse, les immigrés naturalisés ont aussi beaucoup plus de chances que leurs homologues non naturalisés de bénéficier d'une formation pratique et d'être recrutés dans le secteur public ${ }^{57}$. Toutefois, les immigrés originaires de pays à faible revenu restent très peu représentés dans le secteur public suisse, même lorsqu'ils ont été naturalisés (Liebig et Von Haaren, 2011).

\footnotetext{
$57 \quad$ En général, en Suisse, les immigrés sont fortement sous-représentés dans le secteur public (c'est-à-dire dans l'administration publique et le système éducatif). Bien qu'il en aille également ainsi dans d'autres pays de l'OCDE, la différence entre la part relative de l'emploi public dans l'emploi total des immigrés et cette part dans l'emploi total des autochtones est particulièrement grande en Suisse (voir annexe, graphique 5). Il est permis de le déplorer dans la mesure où, en recrutant des immigrés, le secteur public montre l'exemple au secteur privé. En outre, le fait que les immigrés trouvent des emplois dans le secteur public améliore leur visibilité dans la vie quotidienne. Enfin, le fait que les immigrés travaillent dans le secteur public peut aider les institutions publiques à mieux cerner leurs besoins et ceux de leurs enfants. Les immigrés qui exercent des professions importantes, par exemple la profession d'enseignant, peuvent aussi servir d'exemple à d'autres, en particulier aux immigrés de la deuxième génération.
} 


\section{L'INTÉGRATION DES ENFANTS D'IMMIGRÉS SUR LE MARCHÉ DU TRAVAIL}

\section{Résultats au regard de l'éducation des enfants d'immigrés en Suisse}

144. Comme on l'a vu à la section I, le bilan global de l'intégration des enfants d'immigrés sur le marché du travail suisse est favorable tant dans les comparaisons entre pays que dans celles avec les enfants d'autochtones. Toutefois, comme on l'a déjà dit, cela tient en partie au fait que les deux tiers des enfants de 20 à 29 ans, nés en Suisse de parents immigrés, ont des parents originaires de pays à revenu élevé et ne semblent pas connaître de difficultés pour s'intégrer sur le marché du travail suisse. Ce n'est pas le cas des enfants nés en Suisse de parents immigrés originaires de pays à faible revenu qui, en moyenne, sont confrontés à des difficultés. Pour déterminer le point de départ des mesures visant à favoriser l'intégration sur le marché du travail de ce groupe défavorisé, il est intéressant de remonter en arrière et de voir comment ce groupe s'intègre dans le système éducatif suisse qui, en principe, le prépare à prendre pied sur le marché du travail.

145. D'une manière générale, on s'attendrait à ce que les résultats au regard du marché du travail des enfants immigrés soient meilleurs là où ils obtiennent de bons résultats sur le plan de l'éducation. Si l'on prend comme indicateur du degré d'intégration les différences de performances en compréhension de l'écrit des élèves âgés de 15 ans, les résultats de l'édition 2009 du Programme international pour le suivi des acquis des élèves (PISA) montrent qu'en Suisse, les enfants d'immigrés affichent de plus fortes disparités de résultats sur le plan éducatif par rapport aux enfants d'autochtones que dans d'autres pays de l'OCDE (voir tableau 10). Les enfants d'immigrés obtiennent des résultats en lecture nettement inférieurs à ceux des enfants d'autochtones, surtout s'ils ne sont pas nés en Suisse mais y ont immigré pendant leur petite enfance. Particulièrement frappantes sont les fortes disparités entre les jeunes immigrés et les enfants d'autochtones (résultats près de deux fois moins bons que la moyenne OCDE), en dépit du fait que les disparités d'éducation des parents entre ces jeunes immigrés et les enfants d'autochtones ne sont pas supérieures à la moyenne OCDE.

146. Compte tenu de ces différences notables en matière de résultats scolaires telles que mesurées par le PISA, les disparités globales assez faibles en matière de résultats sur le marché du travail des enfants d'immigrés ont de quoi surprendre. Avec des données longitudinales sur le processus de passage de l'école à l'emploi, il est possible de mesurer le profil de carrière et les résultats ultérieurs des enfants d'immigrés de manière plus approfondie en les comparant à ceux des enfants d'autochtones. Ces données sont disponibles pour la Suisse sous la forme de l'enquête par panel PISA/TREE qui est la principale source de données sur laquelle est basée l'analyse qui suit. Cette enquête suit les participants à l'enquête PISA 2000 en Suisse pendant le processus de passage de l'école à l'emploi, la dernière vague d'enquête datant de 2010. L'encadré 5 donne des informations plus détaillées sur cet ensemble de données unique en son genre. 
Tableau 10 : Différences de points PISA de résultats en lecture pour les enfants d'immigrés comparé aux enfants de parents nés dans le pays, 2009

\begin{tabular}{|c|c|c|c|c|c|c|}
\hline & \multicolumn{2}{|c|}{ Non ajusté } & \multicolumn{2}{|c|}{ Ajusté } & \multicolumn{2}{|c|}{$\begin{array}{l}\text { Différences en nombre d'années de } \\
\text { scolarisation des parents comparé aux } \\
\text { enfants de parents nés dans le pays }\end{array}$} \\
\hline & $\begin{array}{c}\text { Enfants d'immigrés } \\
\text { nés dans le pays }\end{array}$ & Jeunes immigrés & $\begin{array}{l}\text { Enfants d'immigrés, } \\
\text { nés dans le pays }\end{array}$ & Jeunes immigrés & $\begin{array}{l}\text { Enfants d'immigrés, } \\
\text { nés dans le pays }\end{array}$ & Jeunes immigrés \\
\hline Australie & -16 & -3 & -19 & -3 & 0.0 & 0.5 \\
\hline Autriche & 55 & 98 & 30 & 69 & -1.2 & -1.9 \\
\hline Belgique & 65 & 71 & 37 & 42 & -1.5 & -1.1 \\
\hline Canada & 5 & 8 & -4 & 7 & -0.4 & 0.6 \\
\hline Danemark & 56 & 79 & 32 & 55 & -1.8 & -0.8 \\
\hline France & 55 & 77 & 24 & 51 & -1.8 & -1.4 \\
\hline Allemagne & 54 & 61 & 31 & 42 & -2.0 & -0.7 \\
\hline Grèce & 33 & 69 & 20 & 36 & 0.2 & -0.6 \\
\hline Irlande & -6 & 36 & 4 & 39 & -0.2 & 0.9 \\
\hline Italie & 45 & 81 & 23 & 54 & -0.2 & 0.0 \\
\hline Luxembourg & 56 & 47 & 22 & 24 & -3.4 & -2.0 \\
\hline Pays-Bas & 46 & 44 & 21 & 7 & -2.4 & -2.3 \\
\hline Nouvelle-Zélande & 28 & 6 & 10 & 15 & 0.1 & 0.8 \\
\hline Norvège & 45 & 60 & 31 & 37 & -0.7 & -0.9 \\
\hline Portugal & 16 & 36 & 9 & 29 & 1.1 & 2.2 \\
\hline Espagne & 26 & 62 & 20 & 44 & -0.1 & 0.0 \\
\hline Suède & 53 & 91 & 31 & 61 & -0.7 & -1.8 \\
\hline SUISSE & 42 & 58 & 21 & 42 & -1.7 & -0.9 \\
\hline Royaume-Uni & 7 & 41 & 11 & 29 & -0.3 & -0.3 \\
\hline États-Unis & 22 & 21 & 0 & -5 & -2.1 & -1.9 \\
\hline OCDE & 25 & 33 & 7 & 14 & -1.5 & -0.9 \\
\hline
\end{tabular}

Note : Les chiffres représentent les points de différence dans les résultats PISA 2009 de compétence en lecture entre les enfants de parents nés dans le pays d'un côté, et les enfants d'immigrés nés dans le pays ou non, de l'autre. Le terme "jeunes immigrés" se réfère aux étudiants qui sont nés à l'étranger et dont les parents sont aussi nés à l'étranger. Le terme "enfants d'immigrés, nés dans le pays" se réfère aux étudiants nés dans le pays dont les deux parents sont nés à l'étranger. "Non ajusté" indique les différences de points dans les notes brutes. "Ajusté" indique les différences après avoir contrôlé le milieu socio-économique des étudiants. Le milieu socio-économique a été construit grâce aux variables suivantes : I'Indice socioéconomique international du statut professionnel (ISEI), le plus haut niveau d'éducation des parents de l'élève, l'indice de richesse familiale, l'indice des ressources pédagogiques au foyer et, l'indice des biens liés à la « culture classique » dans le foyer. "OCDE" est la moyenne de tous les pays pour lesquels les données sont disponibles. Les valeurs négatives signifient que les enfants d'immigrés ont de meilleurs résultats que ceux de parents nés dans le pays. Les différences qui ne sont pas, statistiquement, différentes de zéro sont en italique.

Source : OCDE, base de données PISA, 2009. 


\section{Encadré 5. L'enquête par panel PISA/TREE}

L'enquête par panel PISA/TREE examine la cohorte qui a participé à l'étude PISA 2000 et a achevé sa scolarité obligatoire la même année. Les élèves de cette cohorte ayant accepté de participer se sont vu envoyer une enquête annuelle afin de suivre leur parcours éducatif ainsi que leur intégration sur le marché du travail. La cohorte est représentative de la Suisse et de ses régions linguistiques. L'ensemble de données qui en résulte est assez riche et permet un éventail assez large d'analyses.

\begin{tabular}{|c|c|c|c|c|c|c|c|c|}
\hline Année de l'enquête & 2000 & 2001 & 2002 & 2003 & 2004 & 2005 & 2006 & 2007 \\
\hline Échantillon brut & 11710 & 6343 & 5944 & 5609 & 5345 & 5060 & 4852 & 4659 \\
\hline Réponses & 6343 & 5528 & 5206 & 4877 & 4679 & 4506 & 4133 & 3979 \\
\hline \multicolumn{9}{|l|}{ parmi lesquelles } \\
\hline Enfants de parents nés dans le pays & $77 \%$ & $78 \%$ & $79 \%$ & $79 \%$ & $79 \%$ & $79 \%$ & $79 \%$ & $80 \%$ \\
\hline $\begin{array}{l}\text { Enfants nés dans le pays, de parents } \\
\text { nés dans un pays à revenus élevés }\end{array}$ & $10 \%$ & $9 \%$ & $9 \%$ & $9 \%$ & $9 \%$ & $9 \%$ & $9 \%$ & $9 \%$ \\
\hline $\begin{array}{l}\text { Enfants nés dans le pays, de parents } \\
\text { nés dans un pays à bas revenus }\end{array}$ & $6 \%$ & $6 \%$ & $6 \%$ & $6 \%$ & $6 \%$ & $6 \%$ & $6 \%$ & $5 \%$ \\
\hline $\begin{array}{l}\text { Jeunes immigrés nés dans un pays à } \\
\text { revenus élevés }\end{array}$ & $1 \%$ & $1 \%$ & $1 \%$ & $1 \%$ & $1 \%$ & $1 \%$ & $1 \%$ & $1 \%$ \\
\hline $\begin{array}{l}\text { Jeunes immigrés nés dans un pays à } \\
\text { bas revenus }\end{array}$ & $3 \%$ & $3 \%$ & $3 \%$ & $3 \%$ & $3 \%$ & $3 \%$ & $3 \%$ & $3 \%$ \\
\hline \% réponses de l'échantillon brut annuel & $54 \%$ & $87 \%$ & $88 \%$ & $87 \%$ & $88 \%$ & $89 \%$ & $85 \%$ & $85 \%$ \\
\hline \% réponses de l'échantillon brut de 2001 & & & $82 \%$ & $77 \%$ & $74 \%$ & $71 \%$ & $65 \%$ & $63 \%$ \\
\hline
\end{tabular}

Source : TREE

Les taux de réponse jusqu'en 2007 (année la plus récente pour laquelle les données ont été publiées) permettent effectivement d'effectuer une analyse distincte de différents groupes d'enfants d'immigrés, mais n'en limitent pas moins la mesure dans laquelle une analyse détaillée peut être effectuée pour les années ultérieures, s'agissant de questions spécifiques. Au fil des ans, le contenu du questionnaire a varié. S'il se concentrait principalement sur l'enseignement secondaire du deuxième cycle lors des trois premières vagues d'enquête, l'accent a par la suite été mis sur le passage à la vie active ou à l'enseignement supérieur au cours des trois dernières années pour lesquelles on dispose de données.

L'échantillon de 2000 est composé d'individus d'âges différents. L'âge médian ainsi que l'âge moyen est de 15 ans mais la fourchette réelle des âges va de 13 à 18 ans.

147. Dès 2000, l'étude PISA révélait un déficit de performance important pour les élèves de parents immigrés à la fin des études secondaires du premier cycle en Suisse ${ }^{58}$. Ce sont les enfants d'immigrés originaires de pays à faible revenu qui présentaient les plus forts handicaps. En outre, les résultats variaient considérablement selon les groupes d'origine : si les notes en lecture du PISA de la population considérée ne présentaient pas de différence significative par rapport à celles des enfants d'autochtones pour les enfants d'immigrés originaires d'Allemagne, de France, d'Autriche ou de Belgique (qu'ils soient nés à l'étranger ou en Suisse), les jeunes immigrés originaires des pays successeurs de l'ex-Yougoslavie présentaient des écarts de résultats de près de 170 points $^{59}$.

148. Une partie de ces disparités de performances scolaires peut s'expliquer par les différences de milieu socioéconomique des parents. Le graphique 14 montre la corrélation entre les notes moyennes en

\footnotetext{
58 Pour en savoir plus sur l'enquête PISA 2000, voir OCDE (2001), par exemple.

59 A noter toutefois que cette population n'avait été scolarisée que quelques années dans le système éducatif suisse.
} 
lecture du PISA et l'indice ISEI moyen du statut socioéconomique parental pour différents groupes d'enfants d'immigrés, la taille des bulles indiquant la taille relative des groupes (sauf pour les autochtones) ${ }^{60}$.

\section{Graphique 14 : Milieu socio-économique des parents et résultats PISA en lecture en 2000, selon l'origine migratoire et le pays d'origine}

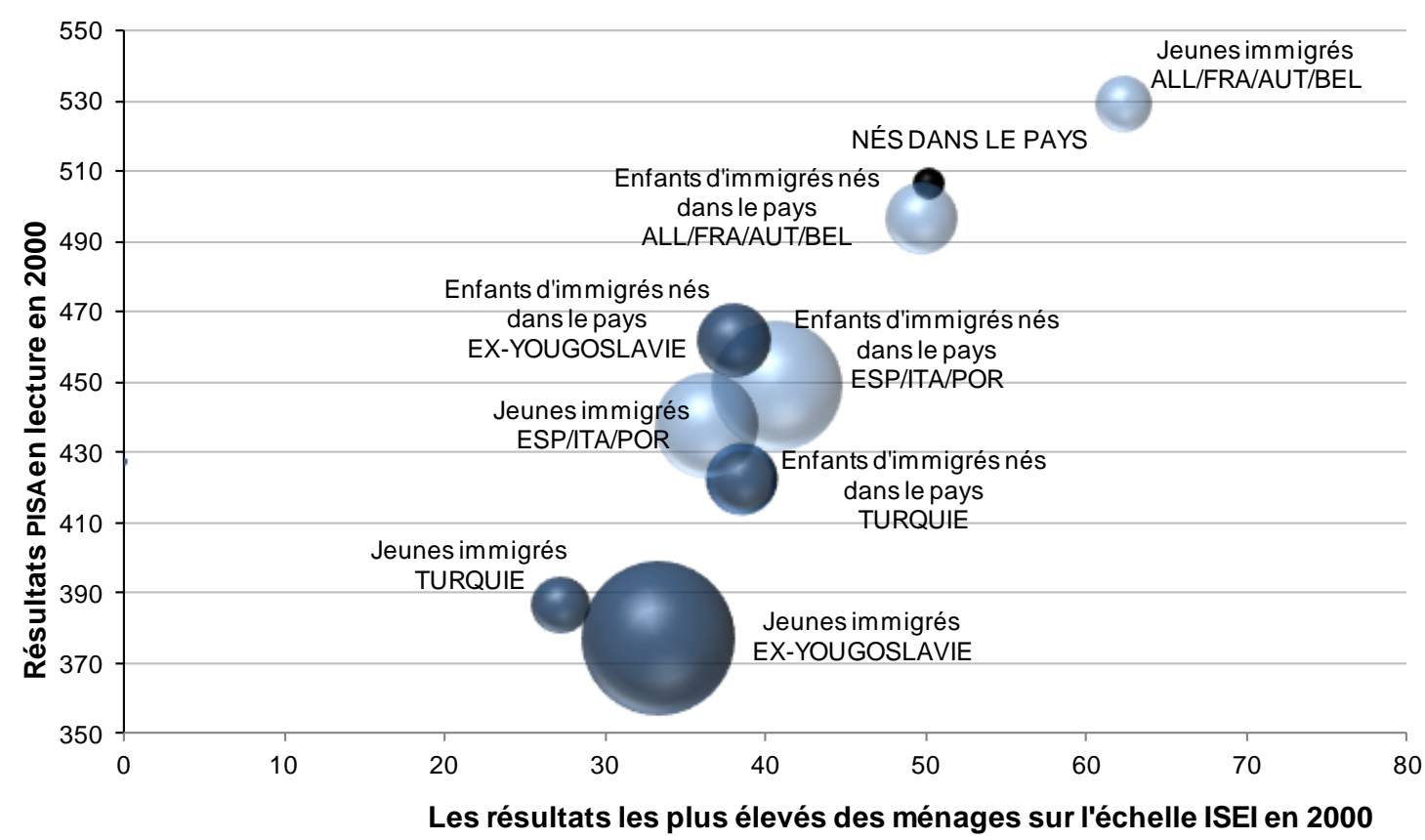

Note : La bulle "nés dans le pays" est la seule à ne pas être dessinée à sa taille relative exacte. Du fait de l'ampleur de ce groupe, sa bulle à taille relative exacte aurait dominé l'ensemble du graphique. Cette bulle ne peut donc être analysée qu'en terme de position et non de taille.

Source : TREE.

149. Comme l'illustre le graphique 14, les enfants immigrés de différents pays d'origine diffèrent généralement de manière significative en fonction du statut social de leurs parents. Les enfants dont les parents sont originaires de pays à revenu élevé tels que l'Allemagne, la France, l'Autriche ou la Belgique affichent un statut socioéconomique moyen comparable à celui des enfants d'autochtones ou, dans le cas des jeunes immigrés, encore plus favorable. En revanche, les enfants de parents originaires de Turquie ou des pays successeurs de l'ex-Yougoslavie ainsi que de pays à revenu élevé tels que l'Espagne, l'Italie ou le Portugal sont généralement issus de familles au statut socioéconomique plus faible. Bien que presque tous les enfants immigrés obtiennent, en moyenne, des résultats scolaires moins élevés d'après l'étude PISA, même après prise en compte du statut socioéconomique, les élèves dont les parents ont un faible statut socioéconomique sont plus défavorisés. Pour l'élaboration des politiques, il est particulièrement intéressant de comprendre comment ces différences, en corrélation avec le contexte migratoire, se traduisent sur le plan de l'éducation et, ultérieurement, de l'emploi.

\section{Éducation des jeunes enfants}

150. Une méthode assez efficace pour lutter contre les handicaps résultant de l'appartenance à un milieu socioéconomique défavorable semble être l'inscription des enfants dans des programmes

60 L'indice socioéconomique international de statut professionnel (ISEI) fait référence au résultat ISEI du père et de la mère. 
d'éducation et d'accueil des jeunes enfants $(E C E C)^{61}$. Les mesures visant les enfants vers l'âge de 3 ou 4 ans semblent particulièrement probantes à cet égard, et le résultat semble même plus bénéfique pour les enfants d'immigrés que pour les enfants d'autochtones. Une récente étude provenant d'Allemagne a montré que la fréquentation de structures d'ECEC augmente les chances d'inscription dans les établissements d'enseignement secondaire les plus exigeants (Écoles de maturité) de plus de $55 \%$ pour les enfants d'immigrés contre $38 \%$ de moyenne globale (Fritschie et Oesch, 2008). De la même façon, une étude française a montré que la fréquentation de structures d'ECEC à l'âge de 3 ans a un effet positif sur les résultats au regard de l'éducation des enfants d'immigrés (Caille, 2001) ${ }^{62}$.

En Suisse, la participation à des programmes d'ECEC a été particulièrement faible, surtout chez les enfants de moins de 4 ans. Parmi ceux âgés de 3 ans, moins de $10 \%$ participent à des programmes préscolaires, ce qui est dû au fait que, dans la plupart des cantons sauf le Tessin, l'ECEC ne commence qu'à l'âge de 4 ans. S'agissant du taux d'inscription des enfants de 4 ans, la Suisse se classe aussi au dernier rang parmi les pays du groupe de comparaison. En 2008, seuls $40 \%$ des enfants de cet âge fréquentent une structure formelle d'ECEC (voir graphique 15). En moyenne, les enfants en Suisse ne suivent que pendant 1.4 an les programmes préscolaires, contre une moyenne de 2.3 ans pour l'ensemble de l'OCDE ${ }^{63}$. On ne dispose pas de chiffres distincts sur les taux d'inscription des enfants issus de l'immigration. Bien qu'il n'y ait pas d'évidence qu'ils soient particulièrement désavantagés à cet égard (voir plus haut), les enfants d'immigrés ont peu de chances d'avoir un taux d'inscription plus élevé que le faible taux relevé chez les enfants de familles non-immigrées.

\section{Graphique 15: Pourcentage d'enfants de 3 et 4 ans pris en charge par un service de garde ou une crèche, dans quelques pays de l'OCDE, 2008}

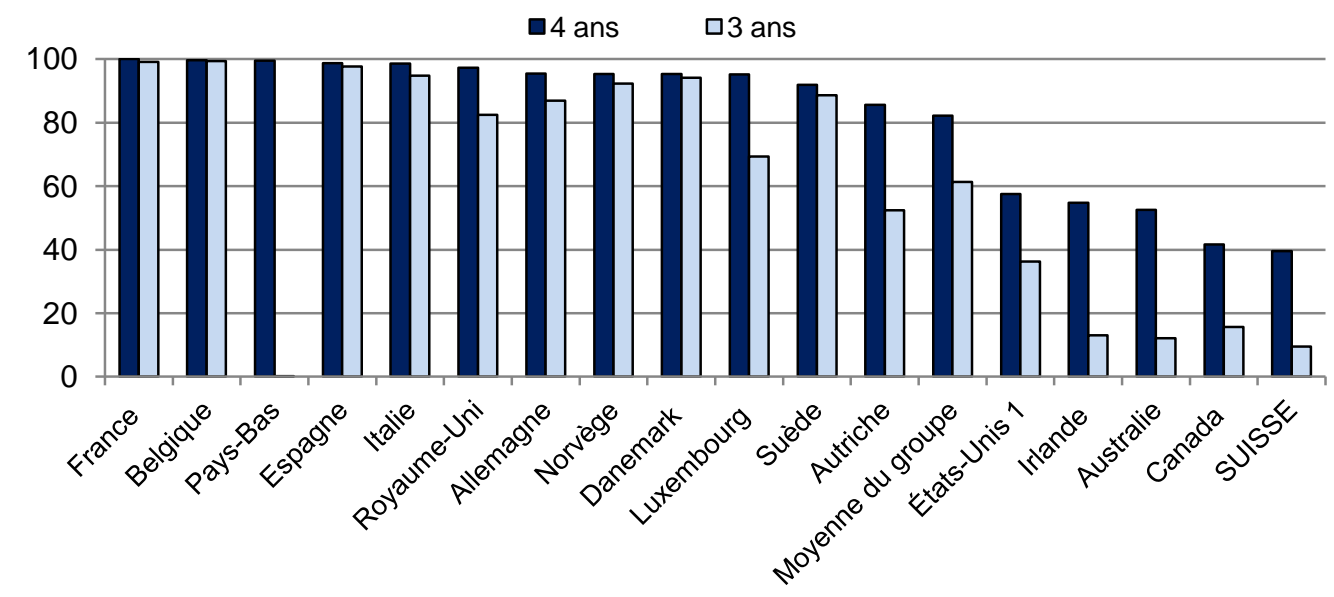

Source : OCDE, Base de données sur l'éducation.

151. Bien que les chercheurs soient largement d'accord sur l'impact bénéfique des programmes d'accueil et d'éducation des jeunes enfants (ECEC) sur le développement cognitif des enfants issus de milieux défavorisés, la question ne suscite l'intérêt du public et l'attention des pouvoirs publics que depuis

Dans toute cette section, le terme «éducation préscolaire » sera utilisé comme synonyme d'éducation et d'accueil des jeunes enfants (ECEC). OCDE (2006). 
peu en Suisse ${ }^{64}$. S'occuper des jeunes enfants à la maison est une tradition de longue date en Suisse, et cela demeure prioritaire par rapport à l'établissement d'une stratégie institutionnelle globale dans l'opinion publique $^{65}$. De fait, les projets de soutien linguistique pour les enfants défavorisés âgés de 0 à 3 ans dans certains cantons germanophones offrent aux mères une formation et une assistance linguistique à la maison, ce qui perpétue le système en place au lieu de favoriser les structures d'ECEC institutionnalisées.

152. Une récente expérience de choix initiée et financée par le Fonds national suisse a montré que la demande de programmes préscolaires ciblant les enfants de moins de 4 ans est en fait bien supérieure à l'offre existant actuellement en Suisse. Au cours de cette expérience, 600 familles dont 150 ayant un chef de famille de nationalité étrangère se sont vu présenter un ensemble de différentes offres fictives de structures d'ECEC à des prix différents (inspirés des prix réels du marché), et il a été demandé aux familles de choisir de façon hypothétique le moyen d'accueil de leurs enfants qu'elles préféraient. Alors que $84 \%$ des participants s'occupaient de leurs jeunes enfants à la maison dans la réalité, seuls $44 \%$ choisissaient cette solution dans l'expérience de réflexion fictive. En outre, la part des participants envisageant d'envoyer leurs enfants à la maternelle $(30 \%)$ était trois fois supérieure à celle des participants qui le faisaient effectivement. Plus remarquable encore, l'étude a révélé que l'offre de maternelles ne permettait de satisfaire que $40 \%$ de la demande à l'époque, laissant entendre que cette demande était assez élastique par rapport aux prix et aux niveaux de revenu (Fonds national suisse, 2005). Le profil de la demande était analogue pour toutes les familles, qu'elles soient ou non issues de l'immigration.

153. Si une initiative a été lancée pour harmoniser la préscolarisation dans les différents cantons en rendant la participation obligatoire à partir de l'âge de 4 ans (comme stipulé dans ce qu'il est convenu d'appeler le «Concordat HarmoS »), il n'y a pas eu d'initiative analogue pour les enfants de 3 ans, âge considéré généralement comme critique pour l'intégration. Les programmes existant pour ce groupe d'âge varient même de façon plus substantielle d'un canton et d'une ville à l'autre. Il existe une multitude de projets à bas seuil d'accès au niveau municipal et cantonal en Suisse, surtout dans les grandes villes comme Berne, Bâle, Zürich et St-Gall. Depuis 2001, par exemple, le gouvernement fédéral a fourni un financement de ce qu'il est convenu d'appeler des Spielgruppen (garderies), qui intègrent des modules de formation linguistique dans leur palette d'activités ${ }^{66}$. Toutefois, il manque à ces mesures un programme commun et une coordination des efforts, et elles ne sont guère institutionnalisées. Bien que beaucoup de ces initiatives locales se concentrent particulièrement sur l'aide aux familles d'immigrés et à leurs enfants, le nombre de familles qui en bénéficient demeure limité (voir Schulte-Haller, 2009). Face à ce morcellement, en 2009, la Commission fédérale des migrations a appelé à l'établissement d'un plan national d'action pour déterminer des conditions et des objectifs communs pour l'ECEC à l'intention des enfants de moins de 4 ans, et inciter à l'harmonisation des diverses offres existant en Suisse. A ce jour, aucun plan de cette nature n'a été mis en œuvre.

64 En 2008, l'UNICEF a publié un rapport qui offre un aperçu des cadres d'ECEC dans 25 pays de l'OCDE. Dans un classement comparatif qui inclut dix normes de bonnes pratiques dans le domaine de l'ECEC, la Suisse se classe dans le peloton de queue car elle ne remplit que trois des dix normes. Au moment de l'établissement du rapport, en 2008, la Suisse n'offrait des structures d'accueil régulées et subventionnées qu'à moins de $25 \%$ des enfants de moins de 3 ans, n'avait pas élaboré de plan national axé sur les besoins des enfants défavorisés, et n'atteignait pas la norme de dépenses de $1 \%$ du PIB par an pour les services à l'enfance. En 2003, seuls deux pays du groupe de comparaison de l'OCDE, l'Irlande et la Corée, consacraient moins de ressources à l'éducation et à l'accueil des jeunes enfants (UNICEF, 2008).

Pour plus de précisions, voir Schulte-Haller (2009).

Entre 2001 et 2007, des ressources ont été fournies par la Commission fédérale des étrangers. Depuis 2008, ces mesures sont financées par l'Office fédéral des migrations. 


\section{Enseignement secondaire du deuxième cycle}

154. Les enfants, en Suisse, entrent généralement à l'école à l'âge de 6 ou 7 ans. Ils suivent l'enseignement général jusqu'à la fin de la scolarité obligatoire, soit pendant 9 ans. Lorsqu'ils achèvent leur neuvième année, ils sont orientés vers différentes filières d'enseignement secondaire du deuxième cycle, ce qui constitue une étape décisive dans leur parcours éducatif. Le type d'enseignement du deuxième cycle choisi à ce stade détermine leur parcours éducatif ultérieur. Ce qu'il est convenu d'appeler les Écoles de maturité/Maturitätsschulen représente la filière la plus prestigieuse. L'admission est sélective et les établissements offrent un enseignement général préparant à l'enseignement supérieur et aboutissant au diplôme d'entrée à l'université du niveau 3A de la CITE (Maturité/Matura). La deuxième option est celle de l'éducation et de la formation professionnelles (EFP), qui conjugue généralement une scolarité à temps partiel et un apprentissage en entreprise. Enfin, les élèves peuvent choisir de continuer des études à plein temps dans des écoles professionnelles, ce qui leur permettra de poursuivre des études ou d'entrer en apprentissage. Mais ils ont rarement recours à cette option.

155. Les élèves qui suivent jusqu'au bout la filière sélective d'une École de maturité obtiennent généralement des résultats assez positifs dans la suite de leurs études ou dans l'emploi. Sur l'ensemble des participants à l'enquête TREE ayant obtenu le diplôme de Maturité (ou une qualification équivalente du niveau 3A de la CITE), dans les sept ans suivant la fin de la scolarité obligatoire, deux sur trois ont ensuite suivi des études supérieures. Quatre pour cent seulement n'étaient ni en emploi, ni en éducation ni en formation (NEET).

156. Une étude plus détaillée montre que les enfants de parents originaires de pays à faible revenu ont beaucoup moins de chances de suivre cette filière avec succès que les enfants d'autochtones (tableau 11), alors que les enfants nés en Suisse de parents immigrés originaires de pays à revenu élevé ne rencontrent pas vraiment d'obstacles. Le milieu socioéconomique des parents est un puissant déterminant des écarts en termes d'achèvement des études. Lorsqu'on tient compte des deux notes du PISA, les différences s'estompent. Si l'on fait intervenir, en outre, le contexte parental, les enfants nés en Suisse de parents immigrés originaires de pays à revenu élevé, de même que les jeunes immigrés provenant de pays à faible revenu, ont encore plus de chances d'obtenir le diplôme de Maturité que les enfants d'autochtones. Cette remarque concerne principalement les jeunes femmes. 
DELSA/ELSA/WD/SEM(2012)2

Tableau 11 : Différences, en point de pourcentage, de probabilité de terminer avec succès l'enseignement secondaire supérieur permettant d'accéder aux études universitaires, selon le genre (cohorte PISA 2000)

\begin{tabular}{|c|c|c|c|c|c|c|c|c|c|}
\hline \multirow[b]{2}{*}{ Par origine migratoire individuelle } & \multicolumn{3}{|c|}{ Modèle 1} & \multicolumn{3}{|c|}{ Modèle 2} & \multicolumn{2}{|c|}{ Modèle 3} & Total Hommes Femmes \\
\hline & & & & & & & & & \\
\hline $\begin{array}{l}\text { Enfants nés dans le pays, de parents } \\
\text { nés d'un pays à revenus élevés }\end{array}$ & -4 & -8 & -2 & $14^{\star}$ & 13 & 15 & $18^{*}$ & 14 & $24^{\star}$ \\
\hline $\begin{array}{l}\text { Enfants nés dans le pays, de parents } \\
\text { nés dans un pays à bas revenus }\end{array}$ & $-24^{\star \star \star}$ & $-25^{\star \star \star}$ & -11 & -8 & -14 & 6 & 2 & -7 & 20 \\
\hline $\begin{array}{l}\text { Jeunes immigrés nés dans un pays à } \\
\text { revenus élevés }\end{array}$ & $-18^{\star \star}$ & -16 & 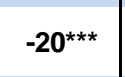 & -5 & -7 & -3 & -4 & -8 & 4 \\
\hline $\begin{array}{l}\text { Jeunes immigrés nés dans un pays à } \\
\text { bas revenus }\end{array}$ & $-25^{\star \star \star}$ & $-24^{\star \star \star}$ & $-28^{\star \star \star}$ & 11 & 5 & 22 & $21^{*}$ & 11 & 35 \\
\hline \multicolumn{10}{|l|}{ Ensemble des enfants d'immigrés } \\
\hline $\begin{array}{l}\text { Parents nés dans un pays à revenus } \\
\text { élevés }\end{array}$ & -6 & -11 & -1 & 7 & 1 & 13 & 9 & -2 & $22^{*}$ \\
\hline $\begin{array}{l}\text { Parents nés dans un pays à bas } \\
\text { revenus }\end{array}$ & $-21^{\star \star \star}$ & $-27^{\star \star \star}$ & -10 & 3 & -11 & 25 & 12 & -8 & $38^{*}$ \\
\hline \multicolumn{10}{|l|}{ Contrôle } \\
\hline \multirow{3}{*}{$\begin{array}{l}\text { Résultats PISA en lecture pour } 2000 \\
\text { Milieu socio-économique } \\
\text { Niveau d'éducation de la mère }\end{array}$} & & & & $\mathrm{x}$ & $\mathrm{x}$ & $\bar{x}$ & $\mathrm{x}$ & $\bar{x}$ & $\bar{x}$ \\
\hline & & & & & & & $x$ & $x$ & $x$ \\
\hline & & & & & & & $x$ & $x$ & $x$ \\
\hline
\end{tabular}

Note : Les chiffres montrent la différence entre les enfants d'immigrés et ceux de parents nés en Suisse. Ils correspondent aux effets marginaux après l'estimation Probit, calculés pour l'échantillon au moyen des variables respectives. Le groupe de référence est celui des personnes nées dans le pays. ${ }^{*},{ }^{* *},{ }^{* * *}$ indiquent un seuil de significativité de $1 \%, 5 \%$, et $10 \%$ respectivement. Dans les quatre premières lignes, les personnes interrogées sont regroupées selon leur propre statut migratoire ainsi que selon le pays d'origine des parents. Les deux lignes suivantes indiquent les résultats pour une analyse où les personnes interrogées sont regroupées, seulement, selon le pays d'origine de leurs parents.

Source : TREE.

157. Compte tenu de la sous-représentation globale des enfants d'immigrés dans la prestigieuse filière de l'enseignement secondaire du deuxième cycle, il convient d'accorder une attention particulière à l'éducation et à la formation professionnelle (EFP) parce que c'est le parcours qu'emprunte la majorité des enfants d'immigrés en Suisse. De fait, les enfants de nationalité étrangère représentent $36 \%$ des élèves inscrits dans des programmes d'EFP aboutissant à des qualifications de niveau 3C de la CITE pourcentage représentant près de deux fois leur part du groupe d'âge dans sa globalité (19\%). Le programme d'EFP le plus important est l'apprentissage, et le fait d'avoir mené à bien son apprentissage semble être immensément bénéfique pour le passage de l'école à l'emploi des enfants immigrés, surtout ceux dont les parents sont originaires de pays à faible revenu (voir tableau 12). Cela réduit nettement le risque de n'être ni en emploi ni en formation, à la fois en termes absolus et par rapport aux autochtones. Cette observation va dans le sens de ce qu'on a pu voir dans d'autres pays de l'OCDE où se pratique la formation en alternance comme l'Autriche, l'Allemagne ou les Pays-Bas [voir OCDE, 2007 ; OCDE, 2008 ; DELSA/ELSA/WP2(2011)6].

158. Bien que les enfants d'immigrés soient assez bien représentés en début d'apprentissage, la probabilité qu'ils aillent jusqu'au bout de leur apprentissage demeure inférieure à celle des enfants d'autochtones (voir tableau 12). On ne sait pas exactement quelles sont les raisons pour lesquelles les enfants d'immigrés « décrochent » pendant leur apprentissage. 
Tableau 12 : Différence de probabilité de terminer un apprentissage, comparaison des enfants d'immigrés avec les enfants de parents nés en Suisse, selon l'origine migratoire, 2007

(cohorte PISA 2000)

\begin{tabular}{|c|c|c|c|c|c|c|c|c|c|c|c|c|}
\hline \multirow[b]{2}{*}{ Origine migratoire } & \multicolumn{3}{|c|}{ Modèle 1} & \multicolumn{3}{|c|}{ Modèle 2} & \multicolumn{3}{|c|}{ Modèle 3} & \multicolumn{3}{|c|}{ Modèle 4} \\
\hline & Total & Hommes & Femmes & Total & Hommes & Femmes & Total & Hommes & Femmes & Total & Hommes & Femmes \\
\hline $\begin{array}{l}\text { Enfants nés dans le pays de parents } \\
\text { immigrés nés d'un... }\end{array}$ & & & & & & & & & & & & \\
\hline pays à revenus élevés & 2 & -3 & 7 & 1 & -3 & 4 & -4 & -11 & 3 & -5 & -10 & 2 \\
\hline à bas revenus & -4 & -7 & 5 & -5 & $-9^{*}$ & 3 & $-17^{\star \star *}$ & $-20^{* \star \star}$ & 1 & $-18^{\star \star \star}$ & $-21^{\star \star \star *}$ & 0 \\
\hline $\begin{array}{l}\text { Jeune immigré nés dans un... } \\
\text { pays à revenus élevés }\end{array}$ & -3 & $-13^{\star}$ & $26^{\star \star *}$ & -3 & $-13^{\star \star}$ & $27^{\star \star \star}$ & $-11^{*}$ & $-19^{\star \star \star}$ & $20^{*}$ & $-10^{\star}$ & $-18^{\star \star \star}$ & $20^{*}$ \\
\hline à bas revenus & -1 & $-9^{\star \star}$ & $16^{* \star *}$ & -3 & $-18^{\star \star \star}$ & $17^{\star \star \star}$ & $-21^{\star \star *}$ & $-25^{\star \star \star}$ & $-14^{\star \star}$ & $-23^{\star \star \star}$ & $-30^{\star \star *}$ & $-13^{\star *}$ \\
\hline $\begin{array}{l}\text { Parents dont le pays d'origine est un pays } \\
\text { à revenus élevés }\end{array}$ & & $-8^{*}$ & $11^{\star \star}$ & & $-8^{\star}$ & 9 & & $-14^{\star \star \star}$ & 6 & & $-14^{\star \star \star}$ & 5 \\
\hline $\begin{array}{l}\text { Parents dont le pays d'origine est un pays } \\
\text { à bas revenus }\end{array}$ & & $-8^{\star \star}$ & $14^{\star \star}$ & & $-14^{* *}$ & $14^{\star \star \star}$ & & $-21^{* \star *}$ & $-11^{*}$ & & $-25^{\star * *}$ & $-10^{*}$ \\
\hline Contrôles & & & & & & & & & & & & \\
\hline $\begin{array}{l}\text { Région } \\
\text { Statut socio-économique des parents } \\
\text { Résultats PISA en lecture } \\
\text { Genre }\end{array}$ & $\mathrm{x}$ & $\mathrm{x}$ & $\mathrm{x}$ & $\begin{array}{l}x \\
x\end{array}$ & $\begin{array}{l}x \\
x\end{array}$ & $\begin{array}{l}x \\
x\end{array}$ & $\begin{array}{l}x \\
x\end{array}$ & $\mathrm{x}$ & $x$ & $\begin{array}{l}x \\
x \\
x \\
x\end{array}$ & $\begin{array}{l}x \\
x \\
x\end{array}$ & $\begin{array}{l}x \\
x \\
x\end{array}$ \\
\hline
\end{tabular}

Note : Les chiffres montrent la différence entre les enfants d'immigrés et ceux de parents nés en Suisse. lls correspondent aux effets marginaux après l'estimation Probit, calculés pour l'échantillon au moyen des variables respectives. Le groupe de référence est celui des personnes nées dans le pays. ${ }^{*},{ }^{* *},{ }^{* * *}$ indiquent un seuil de significativité de $1 \%, 5 \%$, et $10 \%$ respectivement.

Source : TREE.

159. Dans l'ensemble, les enfants d'immigrés affichent une plus forte tendance à quitter l'école sans aucun diplôme du secondaire du deuxième cycle que les enfants d'autochtones (voir graphique 6). Environ $25 \%$ des élèves dont les parents sont originaires de pays à faible revenu ayant participé à l'étude PISA 2000 n'ont obtenu aucun diplôme de ce niveau dans les sept ans suivant la fin de la scolarité obligatoire, contre $10 \%$ seulement des enfants d'autochtones. La proportion parmi les enfants de parents originaires de pays à haut revenu s'élève à environ $15 \%$ pour les enfants nés en Suisse, et même à $25 \%$ pour les jeunes immigrés, ce qui est donc beaucoup plus que pour les enfants d'autochtones.

\title{
Graphique 16 : Proportion de personnes n'ayant pas atteint le second cycle de l'enseignement secondaire, sept ans après la fin de la scolarité obligatoire (cohorte PISA 2000)
}

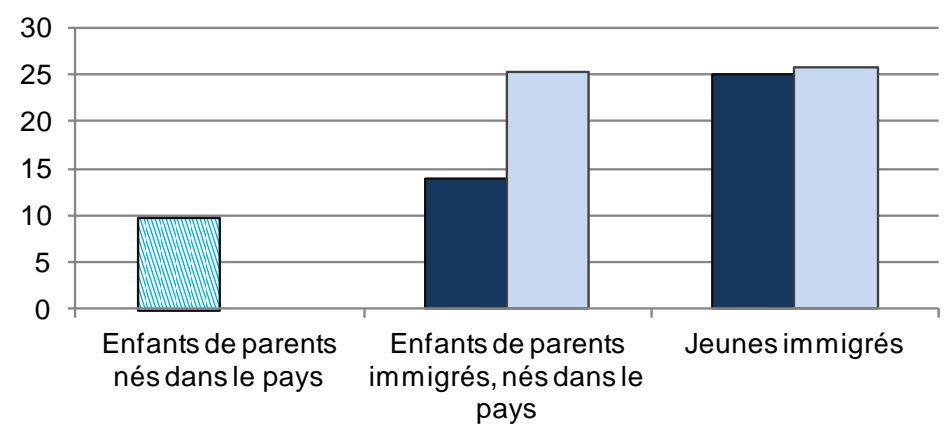

\author{
Parent nés dans... \\ un pays à revenus élevés \\ $\square$ un pays à bas revenus
}

Source: TREE.

160. Ces disparités peuvent s'analyser au regard des résultats scolaires antérieurs et du milieu social des parents. Dans une analyse par régression, les différences tendent à devenir non significatives quand les 
deux facteurs sont pris en compte ${ }^{67}$. Cela explique en partie les résultats étonnamment médiocres des enfants dont les parents sont originaires de pays à revenu élevé, les parents issus des grands pays d'origine que sont l'Espagne, le Portugal ou l'Italie ayant généralement un statut socioéconomique assez faible en moyenne (voir graphique 14 ci-dessous).

161. Un groupe particulièrement important pour l'action publique est celui des enfants qui ne sont ni en emploi, ni en éducation ni en formation (appelé groupe NEET). Les enfants d'immigrés originaires de pays à faible revenu, même après prise en compte de leurs résultats à l'enquête PISA et du niveau d'études atteint, ont une probabilité beaucoup plus forte que les enfants d'autochtones de figurer dans le groupe NEET sept ans après la fin de la scolarité obligatoire (tableau 13). Les écarts sont particulièrement importants pour les hommes et, de fait, leurs piètres résultats à l'enquête PISA n'expliquent pas totalement leur handicap.

Tableau 13 : Différence de probabilité de n'être ni dans le système éducatif ni dans en emploi (NEET), sept ans après la fin de la scolarité obligatoire, (cohorte PISA 2000)

\begin{tabular}{|c|c|c|c|c|c|c|c|c|c|c|c|c|}
\hline & \multicolumn{3}{|c|}{ Modèle 1} & \multicolumn{3}{|c|}{ Modèle 2} & \multicolumn{3}{|c|}{ Modèle 3} & \multicolumn{3}{|c|}{ Modèle 4} \\
\hline & Total & Hommes & Femmes & Total & Hommes & Femmes & Total & Hommes & Femmes & Total & Hommes & Femmes \\
\hline \multicolumn{13}{|l|}{$\begin{array}{l}\text { Enfants d'immigrés dont les } \\
\text { parents sont nés dans... }\end{array}$} \\
\hline un pays à revenus élevés & 2 & $14^{\star \star \star}$ & 1 & 1 & $13^{\star \star \star}$ & 0 & 1 & 5 & $-3^{*}$ & 1 & 4 & $-3^{*}$ \\
\hline un pays à bas revenus & $17^{* * *}$ & $13^{\star \star \star}$ & $20^{\star \star \star}$ & $13^{\star \star \star}$ & $12^{\star \star \star}$ & $8^{* *}$ & $13^{\star \star *}$ & $18^{\star \star \star}$ & $9^{* *}$ & $27^{\star \star \star}$ & $36^{* \star *}$ & $-7^{\star \star *}$ \\
\hline Apprentissage & & & & & & & 1 & $-5^{\star \star \star}$ & $6^{\star \star \star}$ & $2^{*}$ & -3 & $5^{\star \star \star}$ \\
\hline $\begin{array}{l}\text { Intéraction apprentissage } \\
\text { * pays à bas revenus }\end{array}$ & & & & & & & & & & $-5^{\star \star *}$ & $-5^{\star \star \star}$ & $-2^{* \star \star}$ \\
\hline Contrôle & & & & & & & & & & & & \\
\hline Résultats PISA en lecture & & & & $\mathrm{x}$ & $\mathrm{x}$ & $\mathrm{x}$ & $\mathrm{x}$ & $\mathrm{x}$ & $\mathrm{x}$ & $\mathrm{x}$ & $\mathrm{x}$ & $\mathrm{x}$ \\
\hline Régions linguistiques & $\mathrm{x}$ & $\mathrm{x}$ & $x$ & $\mathrm{x}$ & $\mathrm{x}$ & $\mathrm{x}$ & $\mathrm{x}$ & $\mathrm{x}$ & $\mathrm{x}$ & $\mathrm{x}$ & $x$ & $\mathrm{x}$ \\
\hline Genre & $\mathrm{x}$ & & & $\mathrm{x}$ & & & $\mathrm{x}$ & & & $\mathrm{x}$ & & \\
\hline
\end{tabular}

Note : Les chiffres montrent la différence entre les enfants d'immigrés et ceux de parents nés en Suisse. Ils correspondent aux effets marginaux après l'estimation Probit, calculés pour l'échantillon au moyen des variables respectives. Le groupe de référence est celui des personnes nées dans le pays. * ${ }^{* *},{ }^{* * *}$ indiquent un seuil de significativité de $1 \%, 5 \%$, et $10 \%$ respectivement. La catégorie "Apprentissage" concerne les enfants qui ont terminé leur formation d'apprenti. L'échantillon est limité aux enfants qui ont obtenu au moins le niveau CITE 3.

Source : TREE.

162. Alors qu'environ $5 \%$ seulement des enfants de parents autochtones ne sont ni en emploi, ni en formation sept ans après la fin de la scolarité obligatoire, c'est le cas de près de $20 \%$ des jeunes dont les parents sont originaires de pays à faible revenu, même parmi ceux qui sont parvenus à obtenir un diplôme du deuxième cycle du secondaire (graphique 17).

$67 \quad$ Les résultats de l'analyse par régression ne sont pas indiqués dans le présent document mais sont disponibles sur demande au Secrétariat. 


\section{Graphique 17 : Proportion de jeunes ni scolarisés ni employés, sept ans après la fin de la scolarité obligatoire, selon l'origine des parents et le niveau d'instruction}

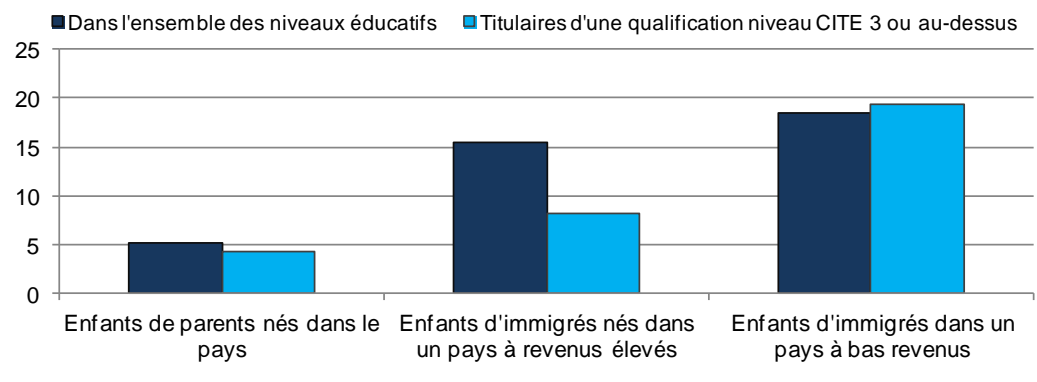

Source : TREE.

163. En résumé, la médiocrité des résultats scolaires à la fin de la scolarité obligatoire et un statut socioéconomique défavorable entraînent la perpétuation du handicap tout au long du deuxième cycle du secondaire pour les enfants d'immigrés originaires de pays à faible revenu, d'où une surreprésentation de ces catégories parmi les jeunes sans diplôme et au sein du groupe des NEET. Trouver les moyens de mieux intégrer les enfants d'immigrés pendant la période préscolaire et pendant les premières années de la scolarité obligatoire et réduire les écarts de performance tels que ceux constatés par l'étude PISA avant la fin de la scolarité obligatoire paraît crucial pour éviter que les enfants d'immigrés ne prennent du retard dans leur parcours scolaire ${ }^{68}$.

\section{Processus de transition de l'école à l'emploi et résultats sur le marché du travail}

164. Pendant le processus de transition de l'école à l'emploi, ce ne sont pas seulement les résultats scolaires antérieurs qui comptent, mais aussi le processus lui-même, c'est-à-dire la durée de la recherche d'un emploi et les filières utilisées pour obtenir un premier emploi.

165. Les données de l'enquête par panel TREE montrent que les enfants nés en Suisse de parents immigrés originaires de pays à revenu élevé connaissent un processus de transition grosso modo analogue à celui des enfants de parents nés en Suisse (voir graphique 18). En moyenne, ils suivent à peu près le même nombre d'années le système éducatif et comptent une part analogue de jeunes en emploi sept ans après la fin de la scolarité obligatoire (entre 40 et $50 \%$ ). Pour leur part, les enfants d'immigrés originaires de pays à faible revenu quittent généralement le système éducatif et prennent un emploi plus tôt. 


\section{Graphique 18: Transition de l'enseignement à l'emploi des enfants de parents nés dans le pays et des enfants d'immigrés nés dans le pays dont les parents sont nés dans un pays à revenus élevés (cohorte PISA 2000)}

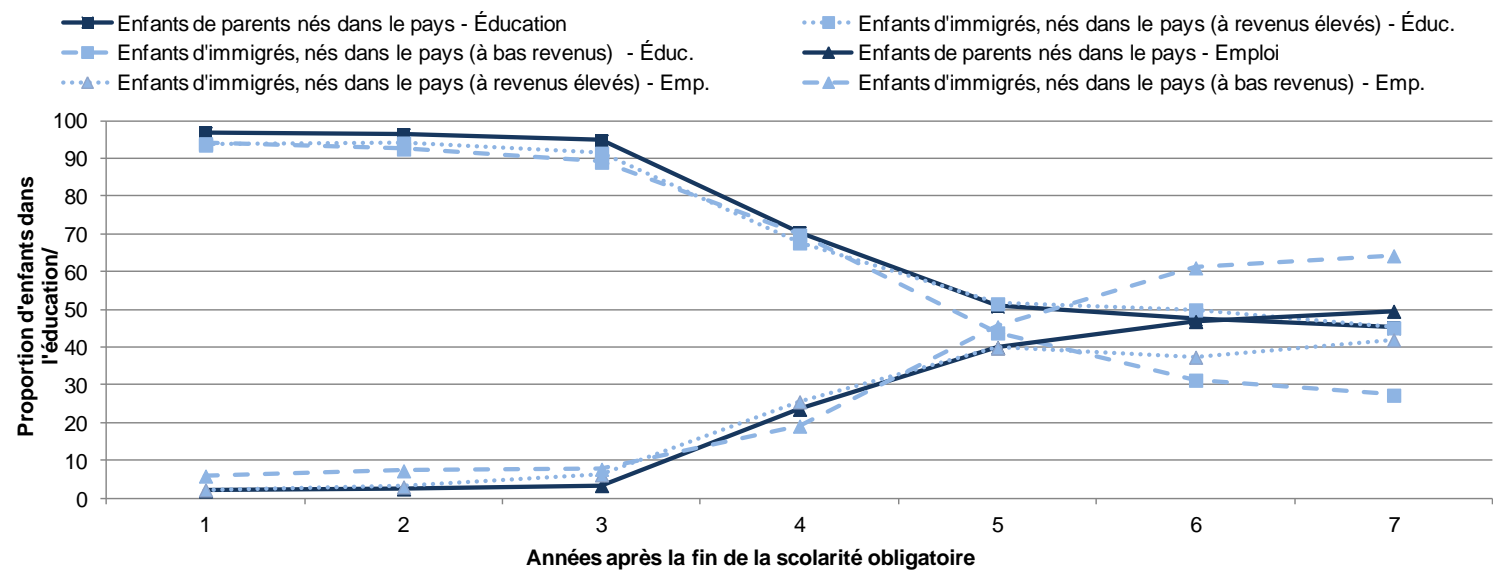

Source : TREE.

166. Lorsqu'on observe uniquement la situation individuelle au regard de l'emploi des participants à l'enquête TREE deux ans après l'obtention du diplôme le plus élevé, il ne semble pas y avoir de disparité de résultats par rapport aux enfants d'autochtones, même pour les enfants d'immigrés originaires de pays à faible revenu ${ }^{69}$. La majorité de ceux ayant obtenu un diplôme deux ans auparavant et qui ne suivent plus d'études à temps plein ont suivi une formation professionnelle. Dotés d'une formation par apprentissage en entreprise, ils ont assez bien géré le processus de transition, ce qui met en évidence le succès de cette filière pour assurer l'emploi des enfants d'immigrés. A cet égard, les réseaux personnels offrant ce type de filière constituent un facteur déterminant du passage de l'école à la vie active, par delà la seule éducation, en volume et en qualité ${ }^{70}$.

167. L'analyse fondée sur des données de l'Enquête suisse sur la population active (ESPA), qui comporte un module spécial sur le passage de l'école à l'emploi, conduit à penser que les filières formelles de recherche d'emploi jouent un beaucoup plus grand rôle pour les enfants nés en Suisse de parents immigrés originaires de pays à faible revenu que pour tout autre groupe. Les écarts sont encore plus prononcés pour les participants à l'enquête TREE (voir graphique 19). En l'occurrence, les enfants d'immigrés originaires de pays à faible revenu semblent avoir beaucoup moins de chances d'avoir trouvé leur emploi par le biais d'une filière informelle. Mais les écarts observés entre les données de l'enquête ESPA et de l'enquête TREE deviennent non significatifs dès lors que l'on prend en compte les études antérieures $^{71}$. Par conséquent, bien que l'enquête sur la population active et l'enquête par panel TREE fassent penser qu'il existe des différences dans les méthodes par lesquelles les enfants d'immigrés

Les élèves du panel diffèrent du point de vue de leur parcours éducatif. Ils alternent éducation et emploi. En raison de la taille de l'échantillon, il est impossible de prendre en compte tous ces facteurs en utilisant des modèles statistiques plus complexes. L'analyse se concentre plutôt sur la situation de chaque répondant au regard de l'emploi au cours de la deuxième année suivant l'obtention du diplôme le plus élevé, pour garantir un solide niveau de comparabilité. A ce stade, les élèves qui choisissent de poursuivre l'enseignement supérieur ont, pour la plupart, intégré les institutions éducatives, et ceux qui choisissent d'entrer dans la vie active ont eu un certain temps pour s'adapter.

La fiabilité des réponses à l'enquête concernant les diplômes obtenus et les dates d'obtention est sujette à caution. Il est donc difficile d'apprécier la représentativité globale du résultat.

Les tableaux de régression correspondants peuvent être mis à disposition sur demande. Mais ces résultats doivent être considérés avec circonspection vu que la taille des échantillons est plutôt réduite. 
originaires de pays à faible revenu sont parvenus à trouver leur premier emploi, ces différences semblent être liées avant tout aux différences de niveau d'études.

\section{Graphique 19 : Canaux par lesquels les 20-29 ans ont trouvé leur premier emploi, selon le contexte migratoire (cohorte PISA 2000)}

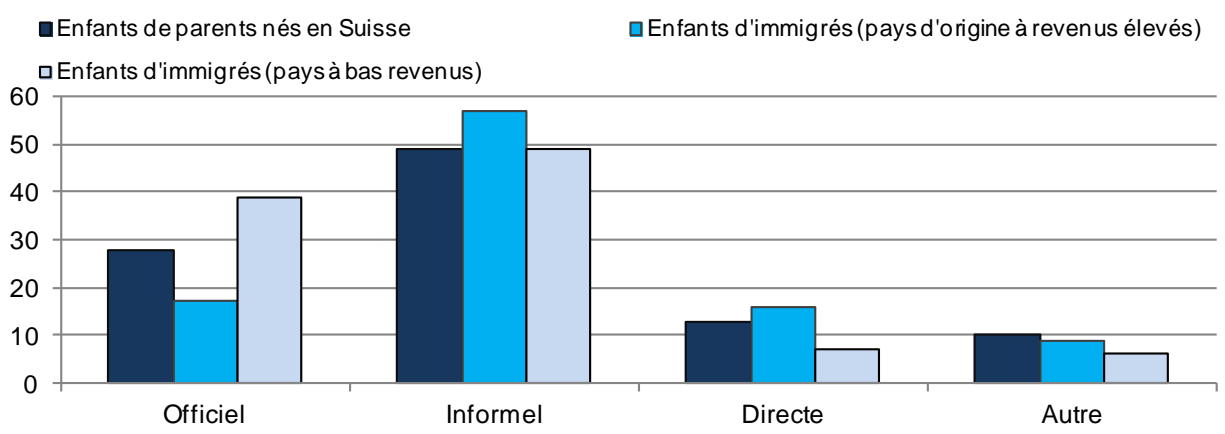

Note : Les enfants d'immigrés nés dans le pays et les jeunes immigrés sont regroupés en raison de contraintes de taille d'échantillon. "Officiel" se réfère à l'usage de l'agence pour l'emploi ou d'une agence d'emploi privée; "Informel" se réfère au réseau personnel; "Directe" se réfère aux candidatures transmises aux employeurs sans intermédiaire.

Source : TREE.

168. Outre l'absence de réseaux, d'autres facteurs peuvent entraver le passage de l'école à l'emploi des enfants d'immigrés, notamment la discrimination (voir plus haut) et la méconnaissance du fonctionnement du marché du travail. Tous ces éléments peuvent conduire à un allongement de la durée de recherche d'emploi. Dans ce cas, on peut s'attendre à ce que les enfants d'immigrés mettent plus de temps que les enfants d'autochtones à trouver un emploi après avoir obtenu leur diplôme de niveau le plus élevé. Il semble que ce soit le cas, sachant que les participants à l'enquête TREE dont les parents sont originaires de pays à faible revenu ont besoin, en moyenne, de deux fois plus de temps pour trouver un premier emploi, et que cet écart demeure relativement robuste même après prise en compte de l'éducation et des scores PISA ${ }^{72}$.

169. S'agissant de l'emploi des 20-29 ans qui ne suivent pas d'études à plein temps actuellement, les enfants nés en Suisse de parents immigrés originaires de pays à revenu élevé semblent s'intégrer assez bien sur le marché du travail local, enregistrant même des résultats légèrement meilleurs que les enfants d'autochtones (voir tableau 14). Plus de $90 \%$ de cette tranche d'âge ont réussi à trouver un emploi. Leur taux de chômage est légèrement inférieur à celui des enfants d'autochtones. Le tableau est différent pour les enfants nés en Suisse de parents immigrés originaires de pays à faible revenu, en particulier pour les jeunes hommes. Ceux-ci affichent un taux d'emploi nettement inférieur et un taux de chômage plus élevé. Les deux mesures diffèrent de 15 à 20 points de pourcentage par rapport aux taux observés pour les enfants d'autochtones. Mais dans le cas des femmes, seuls de légers désavantages sont observés.

\footnotetext{
72 Ces résultats doivent être interprétés avec circonspection. Non seulement les échantillons sont petits, mais il existe aussi un problème de sélectivité des échantillons. On peut, par exemple, supposer que les individus qui ont cherché plus longtemps un emploi n'ont pas du tout intégré le marché du travail. Les personnes ayant suivi un enseignement supérieur auront sans doute mis moins de temps pour trouver un emploi. Or les enfants d'immigrés sont sousreprésentés dans ce groupe. Un tel biais pourrait conduire à une surestimation de l'écart. A noter que la durée de la recherche d'emploi est assez courte en Suisse : elle n'est, en moyenne, que de trois mois environ pour les enfants d'autochtones.
} 
DELSA/ELSA/WD/SEM(2012)2

Tableau 14 : Taux d'emploi et taux de chômage, selon l'origine migratoire et le genre, 20-29 ans, non scolarisés, 2009

\begin{tabular}{|l|cc|cc|}
\hline & \multicolumn{2}{|c|}{ Taux d'emploi } & \multicolumn{2}{c|}{ Taux de chômage } \\
& Hommes & Femmes & Hommes & Femmes \\
\hline $\begin{array}{l}\text { Enfants de parents nés dans le pays } \\
\text { Enfants d'immigrés, nés dans le pays dont les parents }\end{array}$ & 89 & 87 & 7 & 7 \\
sont nés : & & & & \\
$\quad \begin{array}{l}\text { dans un pays à revenus élevés } \\
\text { dans un pays à bas revenus }\end{array}$ & 4 & 5 & -1 & -2 \\
\hline
\end{tabular}

Note: Les chiffres pour les enfants d'immigrés indiquent la différence, en point de pourcentage, entre eux et les enfants nés de parents nés dans le pays. Un taux d'emploi négatif signifie que les enfants d'immigrés ont un taux d'emploi plus bas que celui des enfants de parents nés dans le pays.

Source : Enquête suisse sur la population active, (Office fédéral de la statistique).

170. Comme on l'a vu précédemment, les résultats beaucoup moins favorables au regard de l'emploi des enfants de sexe masculin nés en Suisse de parents immigrés originaires de pays à faible revenu ne peuvent s'expliquer en totalité par le risque plus élevé de ne pas terminer leurs études secondaires du deuxième cycle. Comme indiqué plus haut, même parmi ceux ayant obtenu un diplôme du deuxième cycle du secondaire au moins dans les sept ans suivant la fin de la scolarité obligatoire, les enfants d'immigrés originaires de pays à faible revenu ont encore beaucoup plus de risques de n'être ni à l'école, ni en emploi (voir tableau 13 ci-dessus).

\section{Intervention des pouvoirs publics pour faciliter la transition de l'école à l'emploi}

171. Compte tenu de la précarité des perspectives d'emploi des jeunes ne possédant pas de diplôme du second cycle du secondaire en Suisse, ainsi que de la surreprésentation des enfants d'immigrés dans ce groupe, il semble particulièrement important que les pouvoirs publics interviennent pour aider à la maitrise du passage du premier cycle au deuxième cycle du secondaire. De fait, la Suisse prévoit une large palette de mesures pour accompagner les jeunes dans ce processus de transition. Ce cadre a été renforcé par l'OFFT depuis 2002 pour mieux prendre en compte les difficultés que rencontrent des jeunes pour accéder à l'apprentissage.

172. Le gouvernement fédéral et les cantons se sont engagés à augmenter la part des personnes âgées de 25 ans et titulaire d'un diplôme de deuxième cycle de $90 \%$ en 2004 à $95 \%$ en 2015. Dans ce but, la plupart des cantons développent actuellement un programme de gestion de la formation professionnelle financé par l'OFFT à concurrence de 35.5 millions de francs suisses jusqu'en 2015. Les cantons doivent établir des structures de gestion mieux à même de coordonner les mesures d'orientation des carrières professionnelles. De plus, les gestionnaires devront identifier les jeunes qui rencontrent des difficultés particulières dans le premier cycle et s'assurer qu'ils obtiendront une qualification en rapport avec le second cycle. Bien qu'il n'existe pas encore de données sur les caractéristiques des participants, on s'attend à ce que les enfants d'immigrés soient fortement représentés dans les groupes cibles, définis comme regroupant des jeunes rencontrant de multiples difficultés pour passer du premier au second cycle ${ }^{73}$.

173. Dans le cadre de la récente modification des dispositions suisses en matière d'éducation et de formation professionnelles, les Cantons ont décidé de baisser la limite du seuil exigé pour réguler le passage de trois à quatre années d'apprentissage (voir OCDE, 2009). Ces programmes d'apprentissage

73 La définition de ces «problèmes multiples» peut varier d'un canton a l'autre (voir OFFT, 2010b). Comme le programme est encore dans sa phase initiale, il n'y a pas encore eu d'évaluation rigoureuse concernant son succès ou le groupe de participants. Une évaluation du développement et de la mise en œuvre du projet a été réalisée par Landert (2011). 
s'étalent sur deux ans et donnent lieu à l'obtention d'un certificat qui permet d'accéder à des emplois moins qualifiés. Les apprentis qui risquent d'être exclus de ces programmes, peuvent bénéficier de conseils individuels, dans le cadre d'actions financées au niveau fédéral. A titre exceptionnel, il est aussi possible d'étendre la période d'apprentissage.

174. Enfin, l'OFFT finance aussi des mesures de transition, qui sont mises en ouvre sous la responsabilité des cantons, afin d'établir des liens entre le niveau d'enseignement primaire de second cycle et la formation professionnelle pour des jeunes qui n'arrivent pas à entrer en apprentissage à la fin de la scolarité obligatoire. Les programmes sont généralement prévus pour durer une année. Ils mettent l'accent sur les compétences de base ou consistent en un stage de formation (pré- apprentissage). Dans ce cadre, des cours de langue et d'intégration, destinés aux jeunes immigrés qui rencontrent d'importantes difficultés linguistiques, sont prévus. Toutes ces mesures pavent le chemin entre formation en apprentissage.

175. En plus des moyens de soutien fournis par l'OFFT, il y a aussi des mesures de politiques d'activation du marché du travail destinées aux jeunes qui sont financées par l'assurance chômage. Cependant les jeunes immigrés ayant accompli une partie de leur scolarité à l'étranger ne sont pas nécessairement susceptibles de bénéficier de toute la gamme de mesures de politique d'activation du marché du travail ${ }^{74}$. Le système d'assurance chômage, cependant, accepte à titre exceptionnel des jeunes qui ont passé au moins la dernière année de leur scolarité obligatoire dans le système d'éducation de la Suisse et les autorisent à participer au semestre de motivation (voir ce programme dans l'encadré 6$)^{75}$. Les Cantons doivent cependant financer à hauteur de $50 \%$ les frais occasionnés par ces participants exceptionnels, qui par ailleurs ne reçoivent aucune allocation de chômage dans ce cadre. Il est possible que certains de ces jeunes soient tentés de chercher un emploi peu qualifié afin de gagner rapidement de l'argent, plutôt que d'investir dans une meilleure éducation, ce qui peut accroître les risques de se retrouver dans la situation des NEET (voir supra). Cette situation semble plus répandue parmi les enfants d'immigrés issus de milieux défavorisés.

\footnotetext{
$74 \quad$ En Suisse, l'éligibilité requiert douze mois de contribution pendant une période de deux années précédant le chômage. Les jeunes qui s'inscrivent au chômage après avoir quitté le système éducatif ne sont exempts de ce règlement que s'ils ont été résidents en Suisse pendant une période de dix ans. Cela reflète l'objectif de promouvoir l'immigration familiale dès le départ, mais a aussi pour effet d'exclure les jeunes immigrants qui sont arrivés à la fin de la scolarité primaire ou plus tard.

Alors que le Semestre de motivation s'adresse en général aux jeunes chômeurs, de nombreux cantons offrent en outre des «semestres de pré-motivation». Ces derniers ciblent des bénéficiaires de l'aide sociale qui rencontrent des problèmes de langue ou d'intégration importants et qui ne sont pas encore prêts ou admissibles à participer au cours du semestre de motivation régulier (Spycher et al., 2007).
} 


\section{Encadré 6. Le Semestre de motivation}

Les Semestres de motivation sont un type de programme actif du marché du travail qui existe en Suisse depuis 1994. Ils sont ciblés sur les jeunes de 15 à 25 ans qui ont fini ou quitté l'école et qui sont sans emploi, soit parce qu'ils n'ont pas trouvé de place en apprentissage, ont abandonné un apprentissage ou n'ont aucun plan de carrière quel qu'il soit. Si le programme n'est pas conçu spécifiquement pour les immigrés, beaucoup de ceux qui répondent aux critères pour y participer sont des enfants d'immigrés.

Le Semestre de motivation vise à combler les déficits de formation formelle, à redonner confiance et à préparer les participants pour des emplois réguliers. Cette formule peut comprendre une formation linguistique. L'accent est mis non pas tant sur les compétences techniques (comme la conduite d'une machine) que sur des compétences plus immatérielles comme la ponctualité, le souci de la qualité et l'aptitude à travailler en équipe. Les programmes sont conçus pour se dérouler sur six mois à temps plein (40 heures par semaine), bien qu'ils puissent être prolongés. Si, généralement, l'apprentissage commence en août, l'inscription à un Semestre de motivation peut intervenir à n'importe quel moment de l'année. Les participants pouvant prétendre à des prestations de chômage continuent de les percevoir pendant le Semestre de motivation alors que ceux qui ne remplissent pas les critères d'admissibilité ne bénéficient d'aucun soutien financier pendant la durée du Semestre.

Ainsi, en 2011, 70 programmes ont été mis en place en Suisse (49 dans les cantons germanophones, 18 dans les cantons francophones et 3 au Tessin). Environ 2000 personnes (soit près de $2.5 \%$ d'une cohorte de jeunes) y participent chaque année, et les dépenses par participant s'élevaient à près de $40000 \mathrm{CHF}$ en 2009, ce qui rend la mesure assez coûteuse. Les Semestres de motivation sont financés par l'assurance chômage ${ }^{76}$. Ils sont sous-traités à des organisations du tiers secteur, généralement des organismes de formation ou des entreprises sociales. Le succès du Semestre de motivation repose en partie sur la relation de confiance qui s'instaure entre l'institut de formation et les employeurs locaux. Des dossiers individuels sont créés pour trouver à chaque élève une place de stage qui est censée déboucher sur un apprentissage. La structure de chaque Semestre de motivation varie, de même que la formation individuelle et le placement offerts aux participants.

A Thoune (canton de Berne), par exemple, une fondation propose un Semestre de motivation depuis 2003. Les participants sont orientés par le Service public de l'emploi, plus particulièrement ceux qui ont un faible niveau d'éducation et sont sans expérience professionnelle antérieure. Sur 53 places et 100 participants (du fait de la durée semestrielle du programme) chaque année, environ la moitié sont des enfants d'immigrés. Le programme consiste en deux journées de travail scolaire (langues, mathématiques, études sociales, sports et connaissances générales) en alternance avec trois jours de travail dans un atelier de découpe de PVC. Les élèves sont incités à commencer dès que possible un stage de 2 à 6 mois dans une entreprise. A la fin du stage, il est demandé aux entreprises de prendre les stagiaires comme apprentis. A Thoune, $60 \%$ des participants finissent pas trouver une place en apprentissage, $15 \%$ sont refusés et $25 \%$ « décrochent » du programme.

Le Semestre de motivation représente donc un investissement significatif et semble permettre d'éviter que les jeunes sans emploi ne sortent du système. II traduit la nécessité d'un soutien individuel pendant les cours et le stage. Le taux de réussite des programmes varie en fonction de la situation économique locale et des caractéristiques des jeunes concernés mais, en moyenne, environ deux tiers des participants entrent en apprentissage ou décident de suivre une autre forme d'éducation après la fin du programme (Duell et al., 2010). Cette mesure semble donc assez efficace.

176. En résumé, les bonnes performances globales des enfants d'immigrés dans le passé ont masqué les problèmes importants auxquels se heurtent les enfants d'immigrés originaires de pays à faible revenu dont les effectifs augmentent rapidement. Un certain nombre de programmes bien conçus sont en place mais on accorde trop peu d'attention aux interventions précoces qui sont cruciales pour les résultats en matière d'éducation et la transition de l'école au travail. origines dans l'article $59 \mathrm{~d}$ de la loi d'assurance-chômage. Le semestre de motivation, cependant, vise à placer des participants en apprentissage plutôt que sur le marché de l'emploi. 


\section{EVALUATION ET RECOMMANDATIONS}

\author{
La Suisse compte une forte \\ proportion d'immigrés, et le bilan \\ global du point de vue de \\ l'intégration sur le marché du \\ travail est favorable.
}

En Suisse, environ $27 \%$ de la population sont des personnes nées à l'étranger, ce qui constitue, comme au Luxembourg et en Australie, une des proportions les plus élevées de la zone OCDE. Globalement, les principaux indicateurs de l'intégration sur le marché du travail des immigrés sont tout à fait favorables dans les comparaisons internationales. La Suisse compte parmi les pays de l'OCDE où les immigrés des deux sexes affichent les taux d'emploi les plus élevés, ce qui vaut aussi pour les enfants nés en Suisse de parents immigrés. Néanmoins, et en dépit du faible taux de chômage global que connait le pays, le chômage de certains groupes d'immigrés n'est pas négligeable, s'agissant notamment de nombre de femmes immigrées avec de jeunes enfants.

Cela tient en partie au fait du quasi L'image plutôt favorable de l'intégration en Suisse par rapport aux plein emploi en Suisse et aussi que autres pays de l'OCDE semble s'expliquer, pour l'essentiel, par deux la plupart des immigrés sont facteurs. Premièrement, la situation globale du marché du travail originaires des pays voisins. suisse est bonne. Deuxièmement, contrairement à la plupart des autres pays de l'OCDE, l'essentiel de l'immigration passée et présente provient de pays à revenu élevé, en particulier de l'Allemagne et de l'Italie voisines qui constituent les deux plus importants pays d'origine. Dans le groupe des pays d'origine à faible revenu, l'ex-Yougoslavie et ses pays successeurs représentent près de la moitié de tous les immigrés, et il faut en outre mentionner $12 \%$ de migrants originaires de Turquie. L'essentiel de la migration a consisté en une migration de travail et de regroupements familiaux liées à cette migration, alors que les migrants pour raisons humanitaires (qui tendent à avoir des difficultés particulières pour s'intégrer sur le marché du travail dans tous les pays) représentent une part plus faible qu'ailleurs. Toutefois, même ce groupe n'est pas négligeable en termes d'effectif et, de fait, la Suisse a été l'un des principaux pays de destination des requérants d'asile dans l'OCDE au cours des deux dernières décennies. 
La Suisse compte de nombreux immigrés récents originaires de l'UE, population qui s'est généralement bien intégrée sur le marché du travail.

Certains signes montrent une dégradation de la situation de l'emploi des femmes immigrées originaires de pays à faible revenu, et c'est un point à suivre attentivement.
Depuis 2002, suite à l'instauration progressive de la liberté de circulation avec l'UE et ses pays membres, la Suisse a vu affluer un grand nombre d'immigrés en provenance de l'Union européenne, et plus de $5 \%$ de la population du pays sont des immigrés arrivés pendant cette période. Au sein de ce groupe, il existe une grande diversité sociodémographique des flux selon les pays d'origine, et les immigrés originaires du Portugal en particulier, aussi, sont confrontés à un fort taux de chômage. Généralement, toutefois, les migrants récents en provenance de l'UE élargie affichent de très bons résultats sur le marché du travail. En particulier, ils occupent en moyenne plus d'emplois correspondant à leur niveau de qualification formelle que les autochtones, ce qui suggère que leurs compétences correspondent bien à la demande de main-d'œuvre.

Un groupe dont les résultats sur le plan de l'emploi ne sont pas seulement médiocres mais se sont dégradés ces dernières années est celui des femmes immigrées originaires de pays à faible revenu, en particulier les nouvelles arrivantes. Cette évolution est inquiétante et requiert un suivi attentif. De fait, les femmes immigrées ont généralement tendance à échapper aux efforts d'intégration déployés en Suisse car elles sont souvent éloignées du marché du travail et ne bénéficient d'aucune prestation. Pour ce groupe, la récente réduction du cofinancement (par le biais de l'assurance chômage) des mesures actives du marché du travail pour les personnes ne bénéficiant pas d'une allocation de chômage pourrait bien avoir pour effet une nouvelle réduction de l'offre déjà limitée de mesures d'intégration, à moins que les services sociaux ne s'y opposent. Contrairement aux migrants humanitaires, les migrants venus par le biais d'un regroupement familial ne bénéficient pas d'une aide à l'intégration standard à leur arrivée, bien qu'ils puissent obtenir un certain soutien sous forme d'une formation linguistique de base. A l'inverse, dans les autres pays de l'OCDE, on prévoit de plus en plus de mesures d'accueil pour les nouveaux arrivants et/ou les activités d'organisations non gouvernementales compensent le manque d'offres générales. Ces mesures et activités occupent une place moindre en Suisse. Comme une meilleure intégration des femmes immigrées sur le marché du travail suisse peut aussi avoir d'importants effets d'entraînement pour leurs enfants, l'élargissement des mesures d'intégration à cette population mériterait d'être pris en considération de manière sérieuse et urgente. 
La situation médiocre des migrants pour raisons humanitaires arrivés récemment appelle des mesures plus structurées en faveur de l'intégration.
Les incitations des cantons $\grave{a}$ l'intégration rapide des migrants pour raisons humanitaires sur le marché du travail demandent à être renforcées.
... et les obstacles juridiques à l'accès au marché du travail qui subsistent devraient être levés.
Un groupe qui enregistre de beaucoup moins bons résultats sur le marché du travail maintenant que dans le passé, et qui enregistre aussi de piètres résultats dans les comparaisons internationales, est celui des migrants pour raisons humanitaires arrivés récemment. Les raisons de cette situation ne sont pas entièrement claires. En tout état de cause, il s'agit d'un groupe ayant besoin de mesures d'intégration ciblées. D'ailleurs, d'autres pays de l'OCDE ont mis en œuvre des programmes d'intégration structurés pour ce groupe. Ces mesures, dont la durée peut aller jusqu'à trois ans, semblent avoir rencontré un certain succès. L'amélioration des résultats des migrants pour raisons humanitaires arrivés récemment dans des pays comme le Danemark et la Norvège, dotés de programmes structurés ciblés sur l'intégration sur le marché du travail, fait penser que la Suisse aurait beaucoup à gagner en s'inspirant de cette approche.

Bien que l'aide sociale relève de la compétence des administrations infra-fédérales, elle est financée par la Confédération au niveau des cantons pendant les cinq premières années de séjour des réfugiés. Pour le groupe important et de plus en plus nombreux des personnes admises à titre provisoire, la Confédération va même jusqu'à offrir un financement pendant les sept premières années, avant que les personnes de ce groupe ne commencent à relever de la responsabilité cantonale/communale. En outre, les personnes admises à titre provisoire reçoivent moins que l'aide sociale ordinaire, et il semble que ce phénomène se soit parfois accompagné d'une baisse des efforts pour intégrer ce groupe. Ceci implique que les cantons ne sont guère incités à intégrer sur le marché du travail les migrants pour raisons humanitaires pendant les cinq à sept premières années. Mais si les immigrés ne sont pas préparés correctement au cours de période, il leur sera extrêmement difficile d'entrer avec succès sur le marché du travail. Or, d'après les données d'observation d'autres pays de l'OCDE, une entrée rapide sur le marché du travail est un déterminant crucial de l'intégration à long terme. Il convient donc de renforcer les incitations à l'intégration rapide des migrants pour raisons humanitaires sur le marché du travail.

Depuis 2008, les migrants pour raisons humanitaires admis provisoirement ont accès au marché du travail sans examen de la situation de l'emploi. Toutefois, contrairement aux autres groupes de migrants, il leur faut toujours un permis de travail, qu'ils obtiennent généralement s'ils parviennent à trouver un employeur disposé à les embaucher. Abolir l'obligation d'obtention d'un permis de travail séparé permettrait de réduire la paperasserie et de faciliter l'intégration sur le marché du travail. Dans tous les cas, les employeurs doivent être mieux informés sur la possibilité d'embaucher ces migrants. 


\section{Le cadre de l'intégration est relativement complexe et opaque...}

Comme, jusqu'à une date récente, l'action fédérale dans le domaine de l'intégration était limitée, la plupart des mesures d'intégration ont été mises en œuvre au niveau cantonal ou municipal, reflétant ainsi le principe de subsidiarité. Même si cela a contribué à une approche flexible de l'intégration, cela a également conduit à une importante différence entre les niveaux de prestations de services selon les cantons. Même si cela est vrai pour beaucoup de politiques en Suisse, et concerne aussi de nombreux services pour les nonimmigrés, cela pose la question pour les migrants pour raisons humanitaires de l'équité horizontale étant donné qu'ils ne peuvent pas choisir librement leur canton de résidence et ne peuvent se permettre qu'une mobilité restreinte sur le territoire.

...ce qui est principalement dî au fait que l'accès aux services d'intégration dépend de nombreux facteurs.

\author{
Le niveau inégal de l'offre \\ de services appelle une plus \\ grande coordination de \\ l'action publique.
}

Le caractère assez complexe et opaque du cadre d'intégration suisse est peut-être son principal défaut. Cela tient essentiellement au fait que l'accès aux services d'intégration dépend d'une multiplicité de facteurs -- canton de résidence, type de permis et durée de séjour, perception (ou non) de prestations d'assurances sociales, et régime d'assurance sociale (chômage, invalidité ou aide sociale) dont, le cas échéant, le migrant relève. Ces différences selon les cantons de résidence concernent également les autochtones, mais à la différence des derniers, les immigrés ne sont pas libres de choisir leur lieu de résidence en raison de restrictions à la mobilité géographique, s'agissant principalement des migrants humanitaires.

Le niveau apparemment inégal d'offre de services d'intégration dans les différents cantons et le fait que de nombreux migrants ne peuvent bénéficier des services généraux appellent davantage de coordination de l'action publique, à l'intérieur des cantons et d'un canton à l'autre. Il faudrait appliquer des normes minimum communes pour s'assurer que les immigrés bénéficient de mesures à la hauteur de leurs besoins, indépendamment de leur lieu de résidence en Suisse, de leur type de permis ainsi que de la nature et de l'importance des prestations/allocations d'assurance qu'ils sont en droit ou non de percevoir. Il conviendrait, en outre, de renforcer l'échange de bonnes pratiques entre les cantons et les municipalités. 
Les subventions salariales semblent Pour que cet échange ait son utilité, une évaluation des mesures qui constituer une mesure d'intégration marchent et de celles qui ne marchent pas constitue un préalable, ce particulièrement efficace pour les qui sous-entend qu'il faut intégrer dans les nouveaux programmes la immigrés, mais ceux-cien recherche et l'évaluation. De fait, en dépit d'une culture de bénéficient rarement. l'évaluation globale bien développée en Suisse, il n'y a guère eu d'évaluation spécifique des politiques du marché du travail concernant les immigrés et leurs enfants. Cela reflète l'approche de la politique d'intégration à travers les services généraux, mais fait l'impasse sur le fait que les effets de la politique active du marché du travail peuvent être différents sur les immigrés et sur les autochtones. Un résultat assez concret des évaluations passées (à la fois en Suisse et dans d'autres pays de l'OCDE) est que les subventions salariales sont particulièrement bénéfiques pour les immigrés. Oui, mais en Suisse, ces derniers bénéficient rarement de cette mesure.

Comme les données maintenant disponibles sont de meilleure qualité, remédier au déficit de recherche devrait faire partie d'une politique d'intégration cohérente.
L'absence d'évaluation spécifique des programmes concernant les immigrés se retrouve également dans la recherche sur l'intégration d'une manière plus générale, ce qui est surprenant vu la présence importante et de longue date des immigrés et de leurs enfants en Suisse. L'absence de recherche quantitative est en partie due à un manque de données. Comme des ensembles de données plus nombreux et de meilleure qualité (y compris des données longitudinales) deviennent progressivement disponibles, remédier au déficit de recherche et d'évaluation des programmes concernant l'intégration sur le marché du travail des immigrés et de leurs enfants devrait constituer un élément important de l'élaboration de politiques plus éclairées et mieux ciblées.

La formation linguistique doit être développée et axée plus clairement sur le marché du travail.
Une évidence au niveau international est que l'apprentissage de la langue peut faciliter l'intégration des immigrés. Il est difficile d'évaluer la formation linguistique en Suisse étant donné qu'elle est dispensée en grande partie par différents services au niveau cantonal, sans guère d'interaction entre eux. Le peu d'informations dont on dispose sur la formation linguistique conduit à penser que celle-ci est moins fréquemment dispensée en Suisse que dans d'autres pays de l'OCDE et qu'elle n'est pas associée à de bons résultats au regard de l'emploi pour ceux qui l'ont suivie. Les raisons de cet état de fait méritent un examen plus approfondi et les mesures correspondantes devraient être prises. En tout état de cause, il semblerait judicieux à la fois d'étendre le champ de la formation linguistique et de donner à la formation linguistique une orientation plus professionnelle. Afin de normaliser la formation linguistique dans toute la Suisse, il serait souhaitable d'envisager un cadre général pour cet apprentissage. 
La naturalisation devrait être facilitée car elle favorise l'intégration des immigrés.

Pour favoriser la mobilité, il conviendrait de revoir les critères de résidence cantonaux et municipaux.
La Suisse s'est dotée d'une des politiques de naturalisation les plus restrictives parmi les pays de l'OCDE, reflétant en cela l'idée que la naturalisation est la garantie d'une intégration réussie plutôt qu'un instrument pour la promouvoir. D'après les données empiriques, la naturalisation va de pair avec de meilleurs résultats sur le plan de l'emploi des immigrés et de leurs enfants en Suisse, en particulier pour les immigrés originaires de pays à faible revenu, qui peuvent ainsi avoir accès à des emplois plus qualifiés et à la fonction publique. Ces constatations et d'autres données longitudinales provenant de pays de l'OCDE suggèrent qu'il y aurait apparemment beaucoup à gagner si l'on facilitait l'accession à la nationalité suisse. En tout état de cause, le maintien des restrictions en vigueur a un coût et il conviendrait d'avoir une action de sensibilisation à cet égard et démontrer les avantages liés à l'obtention de la nationalité suisse pour les migrants qui peuvent y prétendre.

Outre le critère fédéral de 12 ans de séjour pour la naturalisation ordinaire, il existe des critères de résidence cantonaux et municipaux. Cela rend l'acquisition de la nationalité plus difficile et peut constituer un frein à la mobilité des immigrés à l'intérieur du pays. Une réforme de la législation suisse sur la naturalisation est en préparation. Elle devrait remédier à quelques-unes des faiblesses les plus significatives de la loi par une harmonisation globale des critères de durée de résidence cantonaux et municipaux, et ramener à huit ans le critère fédéral de durée de séjour. Toutefois, même si la réforme prévue est votée, le système suisse resterait restrictif dans les comparaisons internationales, en particulier pour les nombreux enfants nés en Suisse de parents immigrés parmi lesquels quelquesuns seulement, actuellement, ont la nationalité suisse.

Les mesures d'intégration doivent La plupart des mesures d'intégration disponibles sont ciblées sur mieux tenir compte des compétences l'emploi peu qualifié. Or l'incidence de la «surqualification » des immigrés. (migrants occupant des postes exigeant un niveau de qualification inférieur à la qualification formelle qu'ils possèdent) est élevée pour les migrants ayant obtenu leurs diplômes dans des pays à faible revenu, et ce constat semble rester valable après prise en compte des mesures objectives de compétences. 
La transparence de l'évaluation et de la reconnaissance des diplômes étrangers doit être améliorée, et toutes les professions doivent être couvertes.

La création d'un guichet unique pour toutes les demandes d'évaluation et de reconnaissance serait utile à cet égard.

\author{
Les offres passerelles et le \\ mentorat pour les migrants \\ qualifiés devraient être mis en \\ auvre à une plus grande échelle.
}

L'évaluation et la reconnaissance des diplômes étrangers semblent être un des points faibles du système suisse. Bien que les frais en jeu soient faibles et le processus assez rapide, le système manque de transparence et n'est pas très bien connu même chez les prestataires de services d'intégration. En outre, pour un certain nombre de professions, il est actuellement impossible d'obtenir une évaluation et une reconnaissance des qualifications et de l'expérience de travail acquises à l'étranger, et ce sont là des lacunes du système qu'il faudrait combler. Il est important d'améliorer le système d'évaluation et de reconnaissance car la grande majorité des immigrés diplômés ont obtenu leurs titres à l'étranger. Le résultat de cette procédure semble être accepté sur le marché du travail parce que les immigrés ayant obtenu une reconnaissance formelle affichent de meilleurs résultats sur le plan de l'emploi. Toutefois, rares sont les immigrés possédant des diplômes de l'enseignement supérieur décernés par des pays non membres de l'EEE qui cherchent effectivement l'évaluation et la reconnaissance de ces diplômes. On ne sait pas très bien si c'est parce qu'ils ne sont pas au courant de cette possibilité ou s'ils sont découragés à l'idée de faire la demande, pensant qu'ils ont peu de chances d'obtenir la reconnaissance.

L'Office fédéral de la formation professionnelle et de la technologie (OFFT) accepte déjà les «demandes préliminaires» de reconnaissance dans toutes les disciplines et à tous les niveaux et fournit des informations sur les organismes compétents. Une nouvelle étape serait la création d'un guichet unique qui accepterait formellement toutes les demandes plutôt que de les renvoyer aux candidats avec un avis sur l'organisme qu'il leur faut contacter. Cette démarche constituerait une avancée importante dans le sens de la transparence. En outre, le fait que la reconnaissance aide à obtenir des emplois de qualité devrait être plus largement diffusé auprès des immigrés.

Il existe peu de formations passerelles pour les immigrés, et l'accent mis sur l'intégration rapide sur le marché du travail tant des réfugiés que des chômeurs de façon générale est en contradiction avec les offres passerelles. En particulier, l'accès à des emplois hautement qualifiés s'effectue souvent via des réseaux auxquels la population immigrée est rarement associée. En l'occurrence, les programmes de mentorat semblent un outil efficace, mais leur ampleur et leur portée restent limitées jusqu'à présent. Il semblerait aussi que les immigrés tireraient grandement avantage d'une offre plus large de validation des acquis, dispositif qui se développe progressivement en Suisse. Il conviendrait de s'assurer que les mesures de validation des acquis en cours d'élaboration touchent effectivement les immigrés et répondent à leurs besoins. 
La forte incidence de la discrimination à l'embauche en Suisse mériterait une plus grande sensibilisation à ce problème.
Des études de testing ont mis en évidence une forte incidence de la discrimination à l'embauche. Elles laissent entendre que pour être invités à un entretien, les enfants d'immigrés ayant fait leurs études en Suisse doivent parfois soumettre jusqu'à cinq fois plus de candidatures que les enfants d'autochtones dont le CV est par ailleurs identique. L'incidence relativement faible du chômage chez les enfants d'immigrés suggère que ces derniers compensent la discrimination en soumettant un plus grand nombre de candidatures. Le degré élevé de discrimination est aussi partiellement masqué par la situation globalement bonne du marché du travail et, dans le débat public, on ne se préoccupe guère du risque de discrimination à l'embauche. Néanmoins, les enfants d'immigrés ont besoin de deux fois plus de temps pour trouver un emploi que les autochtones affichant les mêmes résultats scolaires. Une plus grande sensibilisation à ce problème est donc importante.

Le cadre juridique et institutionnel Le cadre juridique et institutionnel de lutte contre la discrimination de lutte contre la discrimination est est largement déficient par rapport à ceux de la plupart des autres largement déficient par rapport aux pays de l'OCDE et, actuellement, la discrimination fondée sur la autres pays de l'OCDE... nationalité n'est pas illégale, excepté pour les immigrés venant de l'UE. En outre, les structures de conseil et d'orientation sont souvent moins bien dotées que dans d'autres pays, et si elles peuvent dispenser des avis juridiques, elles ne peuvent pas entreprendre d'action en justice. En conséquence, le nombre de dossiers qu'elles traitent est négligeable au regard du niveau de la discrimination révélé par les études de testing.

...et le renforcement du cadre devrait être complété par d'autres mesures.
Il est donc important de renforcer à la fois le cadre juridique et le cadre institutionnel de lutte contre la discrimination et de les faire connaître plus largement aux immigrés. Cette réforme pourrait être complétée par des mesures plus volontaristes en faveur de la diversité, visant à la diversification des filières de recrutement par les employeurs, ce qui fait défaut actuellement en Suisse. Le secteur public, dans lequel les immigrés sont sous-représentés, doit aussi participer à ce mouvement. Des outils de diversité tels que les CV anonymes, les labels «diversité » et les conseils en diversité ont récemment été mis en œuvre dans un certain nombre de pays de l'OCDE. 
Le système de l'apprentissage semble bien fonctionner pour les enfants d'immigrés mais les faibles taux d'achèvement des formations méritent plus d'attention.
Le Programme international de l'OCDE pour le suivi des acquis des élèves (PISA) a montré qu'en Suisse, les enfants d'immigrés affichent à l'âge de 15 ans des résultats scolaires bien moins bons que ceux des enfants d'autochtones, et que les écarts y sont supérieurs à ceux observés dans beaucoup d'autres pays de l'OCDE. L'analyse longitudinale tend à indiquer que le système scolaire et de formation réussit néanmoins à donner ultérieurement à ces enfants d'immigrés des qualifications relativement bonnes, et le système d'apprentissage semble marcher particulièrement bien pour eux. Cependant, les enfants d'immigrés ont moins de chances de terminer leur apprentissage. Il faudrait étudier plus avant ce phénomène et prendre les mesures correctives qui s'imposent.

Les enfants d'immigrés semblent
avoir plus de problèmes maintenant

Le mécanisme du passage de l'école à l'emploi fonctionne bien pour que par le passé, et y remédier devrait devenir une priorité de l'action publique.

Tous les jeunes immigrés doivent bénéficier de l'égalité d'accès aux politiques actives du marché $d u$ travail pour les jeunes. la plupart des enfants d'immigrés. En même temps, certains signes montrent que les cohortes récentes d'enfants d'immigrés ont aujourd'hui plus de difficultés que les précédentes, et beaucoup d'enfants d'immigrés nés à l'étranger enregistrent des résultats moins favorables. Cette remarque concerne en particulier les enfants d'immigrés dont les parents sont originaires de pays à faible revenu, qui se trouvent souvent ni en emploi, ni en éducation ni en formation (NEET) quelques années après avoir quitté l'école. Comme les enfants d'immigrés des pays à faible revenu sont aujourd'hui nombreux à entrer dans la vie active, il est important que les pouvoirs publics fassent une priorité de l'amélioration de leurs résultats.

Un vaste cadre de mesures existe pour lisser le processus de passage de l'école à l'emploi des jeunes ayant besoin d'un soutien. Toutefois, l'admissibilité à la plupart des mesures de politique active du marché du travail est souvent limitée aux personnes présentes sur le territoire depuis plus de 10 ans, ce qui exclut de nombreux jeunes immigrés. De plus, les jeunes immigrés bénéficiant de ces programmes, notamment le programme dit «Semestre de motivation», ne perçoivent pas d'allocations de chômage (à la différence des enfants nés en Suisse), et peuvent ainsi être tentés d'entrer directement sur le marché du travail et de se contenter d'un emploi peu qualifié. Ils risquent alors de se retrouver ultérieurement ni en emploi, ni en éducation, ni en formation (NEET), ce qui semble être souvent le cas. Une première mesure pour relever cet important défi serait de s'assurer que les enfants immigrés aient les mêmes incitations à participer à des programmes que les enfants autochtones. 
Il convient d'améliorer l'éducation Un domaine qui semble impliquer des avantages particuliers pour les et l'accueil des jeunes enfants, enfants d'immigrés est l'éducation préscolaire, comme tendent à en se concentrant notamment sur l'indiquer les recherches effectuées dans un certain nombre de pays les enfants d'immigrés défavorisés. de l'OCDE. Toutefois, l'éducation préscolaire est actuellement largement sous-développée en Suisse, en particulier pour les enfants de trois et quatre ans, âges critiques pour l'intégration. La fourniture plus large et mieux ciblée d'une éducation préscolaire à ces âges ainsi que des mesures précoces de soutien linguistique sembleraient présenter des avantages importants. N'en bénéficieraient pas seulement les enfants d'immigrés eux-mêmes mais aussi leurs mères, pour lesquelles le lien entre l'emploi et le fait d'avoir des enfants est beaucoup plus fort que pour les mères autochtones. 
DELSA/ELSA/WD/SEM(2012)2

\section{BIBLIOGRAPHIE}

Avenir Suisse (ed.) (2008), Die neue Zuwanderung. Die Schweiz zwischen Brain-Gain und Überfremdungsangst, Verlag Neue Zürcher Zeitung, Zürich.

BASS (2006), Ausländer/innen, Erwerbslosigkeit und Arbeitslosenversicherung, Seco Publikation Arbeitsmarktpolitik No. 16., State Secretariat for Economic Affairs, Bern.

Becker, L., T. Liebig et A. Sousa-Poza (2006), "Migration Policy and Industrial Structure: The Case of Switzerland”, International Migration, Vol. 46, No. 2, pp. 81-107.

Caille, J.P. (2001), "Scolarisation à 2 ans et réussite de la carrière scolaire au début de l'école élémentaire", Éducation \& formations, Vol. 60, pp. 7-18.

Card, D. (2004), “Is the New Immigration Really so Bad?", The Economic Journal, Vol. 115, No. 507, pp. 300-323.

CFR (1996), Stellungnahme der Eidg. Kommission gegen Rassismus zum Drei-Kreise-Modell des Bundesrats über die schweizerische Ausländerpolitik, Bern.

Conseil fédéral (2010), Bericht zur Weiterentwicklung der Integrationspolitik des Bundes, Bern.

Cueni, D. et Sheldon, G. (2011), Arbeitsmarktintegration von EU/EFTA-Bürgerinnen und Bürgern in der Schweiz. Studie im Auftrag des Bundesamtes für Migration. Forschungsstelle für Arbeitsmarkt- Und Industrieökonomik, Bâle.

de Coulon, A. et Y. Flückiger (2000), "Analyse économique de l'intégration de la population étrangère sur le marché suisse du travail", in P. Centlivres et I. Girod (eds.), Les défis migratoires: actes du colloque CLUSE. Les défis migratoires à l'aube du troisième millénaire, Seismo, Zurich, pp. 109 119.

de Coulon, C. et K. Gäuman K (2011); Rapport SOPEMI 2009, Office fédéral des migrations, Berne.

D'Amato, G. (2009), “Switzerland: A Multicultural Country Without Multicultural Policies?", in S. Vertovec et S.Wessendorf (eds.), The Multiculturalism Backlash, Routledge, London.

Duell, N., P. Tergeist, U. Bazant et S. Cimper (2010), Activation Policies in Switzerland, OECD Social Employment and Migration Working Paper No. 112, Paris.

Fibbi, R. (2005), "Mesures de lutte contre les discriminations à l'embauche“, Forum suisse pour l'étude des migrations et de la population, Neuchâtel.

Fibbi, R., et al. (2010), Les Portugais en Suisse, Study commissioned by the Swiss Federal Office for Migration.

Fibbi, R., M. Lerch et P. Wanner (2006), "Unemployment and Discrimination against Youth of Immigrant Origin in Switzerland - When the Name Makes a Difference“, Journal of International Migration and Integration, No. 7, pp. 351-366. 
Fibbi, R., B. Kaya et E. Piguet (2004), Nomen est omen: Quand s'appeler Pierre, Afrim ou Mehmet fait la différence, Direction du programme PNR43 - FNRS, Bern.

Fibbi, R., B. Kaya et E. Piguet (2003): Le passeport ou le diplôme ? Étude des discriminations à l'embauche des jeunes issus de la migration. Neuchâtel: Forum suisse pour l'étude des migrations Rapport de Recherche 31.

Fritschi, T. et T. Oesch (2008), Volkswirtschaftlicher Nutzen von frühkindlicher Bildung in Deutschland. Eine ökonomische Bewertung langfristiger Bildungseffekte bei Krippenkindern, étude commandée par la Fondation Bertelsmann.

Gerfin, M. et M. Lechner (2002), "A Microeconometric Evaluation of the Active Labour Market Policies in Switzerland“, Economic Journal, No. 112, pp. 854-893.

KEK-CDC Consultants et ODM (2008), Arbeitsmarktintegration von Flüchtlingen und Vorläufig Aufgenommenen - Studie über erfolgversprechende Faktoren, Bern.

Körner, H. (1990), Internationale Mobilität der Arbeit, Wissenschaftliche Buchgesellschaft, Darmstadt.

Lalive, R., J.C. van Ours et J. Zweimueller (2002), The Impact of Active Labour Market Programs on the Duration of Unemployment, Institute of Empirical Research in Economics, University of Zurich, Working Paper No. 41.

Landert, Charles (2011), Projet national case management «Formation professionnelle »: Rapport sur l'évaluation de la mise en œuvre, Bern.

Liebig, T. (2009), Jobs for Immigrants: Labour Market Integration in Norway. OECD Social, Employment and Migration Working Paper No. 94, Paris.

Liebig, T. (2002), Switzerland's immigration experiences: lessons for Germany?, Research Institute for Labour Economics and Labour Law, University of St. Gallen, Discussion Paper No. 76, St. Gallen.

Liebig, T. et F. von Haaren (2011), "Nationalité et integration socio-économique des immigrés et de leurs enfants : vue d'ensemble dans les pays de l'Union européenne et de l'OCDE" in OCDE (ed.), La naturalisation : un passeport pour une meilleure integration des immigrés ?, Editions de l'OCDE, Paris.

Liebig, T. et S. Widmaier (2010), "Overview - Children of Immigrants in the Labour Markets of OECD and EU Countries", in OECD (ed.), Equal Opportunities? The Labour Market Integration of the Children of Immigrants, Editions de l'OCDE, Paris.

Mahnig, H. (ed.) (2005), Histoire des politiques d'immigration, d'intégration et d'asile en Suisse, Seismo, Zurich.

Nekby, L. (2008), Active Labor Market Programs for the Integration of Youths and Immigrants into the Labor Market - The Nordic Experience, Report for the UN Economic Commission for Latin America and the Caribbean (ECLAC), mimeographed.

Niederberger, J.M. (2005), "Le développement d'une politique d'intégration suisse“, in H. Mahnig (ed.), Histoire des politiques d'immigration, d'intégration et d'asile en Suisse, Seismo, Zurich, pp. 255-287. 
Niederberger, J.M. (2004), Ausgrenzen, Assimilieren, Integrieren - Die Entwicklung einer schweizerischen Integrationspolitik, Seismo, Zurich.

OCDE (à paraître), Études économiques de l'OCDE : Suisse 2011, Editions de l'OCDE, Paris.

OCDE (2011a), La naturalisation : un passeport pour une meilleure intégration des immigrés ?, Editions de l'OCDE, Paris.

OCDE (2011b), Perspectives des migrations internationales 2011, Editions de 1'OCDE, Paris.

OCDE (2010a), Equal Opportunities? The Labour Market Integration of the Children of Immigrants, Editions de l'OCDE, Paris.

OCDE (2010b), Études économiques de l'OCDE : Suisse 2009, Editions de l'OCDE, Paris.

OCDE (2009), Learning for Jobs: Switzerland, Editions de l’OCDE, Paris.

OCDE (2008a), Perspectives des migrations internationales, Editions de l'OCDE, Paris.

OCDE (2008b), Les migrants et l'emploi (Vol. 2), Editions de l'OCDE, Paris.

OCDE (2008c), Perspectives de l'emploi, Editions de l'OCDE, Paris.

OCDE (2007), Jobs for Immigrants (Vol. 1), Edition de l'OCDE, Paris.

OCDE (2006a), Petite enfance, grands défis II : Éducation et structures d'accueil, Editions de l'OCDE, Paris.

OCDE (2006b), Études économiques de l'OCDE : Suisse 2007, Editions de l'OCDE, Paris.

OCDE (2000), PISA - Mesurer les connaissances et les compétences des élèves. Lecture, mathématiques et science : l'évaluation de PISA 2000, Editions de l'OCDE, Paris.

ODM (2007), Report Integration Measures, Bern.

OFFT (2010a), Validation of Achievements in Education, Bern.

OFFT (2010b), Case management «Formation professionnelle » : Rapport de monitorage 2010 : état du projet, Bern.

OFIAMT et OFE (1991), Rapport sur la conception et les priorités de la politique suisse des étrangers pour les années 1990, Bern.

Piguet, E. (2009), L'immigration en Suisse - soixante ans d'entrouverture, 2éme édition, Presse polytechniques romandes - Collection "Le Savoir Suisse", Lausanne.

Raymann, U. (2003), Meinungen und Einstellungen gegenüber Ausländerinnen und Ausländern in der Schweiz, Trend- und Vertiefungsbericht, GfS-Forschungsinstitut, Zürich.

Sancar-Flückiger, A. (1999), "Integrationsleitbilder und Integrationspolitik: zur kontroversen LeitbildDebatte in Zürich, Bern und Basel", in H. Dietrich (ed.), Flüchtlinge, Migration und Integration, Widerspruch, Zürich, pp. 137-145. 
Schönenberger, S. et R. Fibbi (2010), Lutte contre les discriminations à l'embauche - Les mesures volontaires mises en oeuvre en Suisse, Forum suisse pour l'étude des migrations, Neuchâtel (Working document commissioned by the Federal Department of the Interior).

Schulte-Haller, M. (2009), Frühe Förderung: Forschung, Praxis und Politik im Bereich der Frühförderung: Bestandsaufnahme und Handlungsfelder, in Swiss Federal Commission for Migration (CFM, ed.), Materialien zur Migrationspolitik, Bern.

Schwarz, H. (1988), Volkswirtschaftliche Wirkungen der Ausländerbeschäftigung in der Schweiz. Rüegger, Chur.

Sheldon, G. (2001), "Foreign Labor Employment in Switzerland: Less Is Not More“, Schweizerische Zeitschrift für Politikwissenschaft, Vol. 7, No. 1, pp. 104-112.

Simeone, L. (2005), Discrimination Testing Based on ILO Methodology, International Labor Office, Geneva, mimeographed.

Swiss National Fund (2005), Familienergänzende Kinderbetreuung in der Schweiz: Aktuelle und zukünftige Nachfragepotenziale, Zürich.

Spycher, S., T. Egger et E. Hüttner (2007), Die Möglichkeiten der sozialen Sicherung bei der Integration von Ausländerinnen und Ausländern, Centre for Labour and Social Policy Studies (BASS, ed.) (Final report for Project No. 405140-69220 in the context of the National Research Programme 51 "Integration and Social Exclusion", Bern.

Steiner, P. (2007), Chronique d'une commission: 37 ans CFE, Commission fédéral des étrangers, Bern.

Steinhardt, M.F., T. Straubhaar et J. Wedemeier (2010), Studie zur Einbürgerung und Integration in der Schweiz: Eine arbeitsmarktbezogene Analyse der Schweizerischen Arbeitskräfteerhebung, Study commissioned by Swiss Federal Office for Migration, Bern.

Swiss Federal Statistical Office (2008), Familien in der Schweiz, Statistischer Bericht, Bern.

Tripartite Agglomeration Conference (2009), Avenir de la politique suisse d'intégration des étrangers, Conférence des gouvernements cantonaux, Bern.

UNICEF (2008), The child care transition. A league table of early childhood education and care in economically advanced countries, UNICEF Innocenti Research Centre (ed.), Innocenti Report Card 8 , Florence.

Van de Voorde, M. et H. de Bruijn (2010), "Mainstreaming the Flemish Employment Equity and Diversity Policy", in OCDE (ed.): Equal Opportunities? The Labour Market Integration of the Children of Immigrants, Editions de l'OCDE, Paris, pp. 229-242.

Weins, C. (2010), "Kompetenzen oder Zertifikate? Die Entwertung ausländischer Bildungsabschlüsse auf dem Schweizer Arbeitsmarkt“, Zeitschrift für Soziologie, Vol. 39, No. 2, pp. 124-139. 


\section{GLOSSAIRE}

AELE

ALL

CFE

CFM

CFR

CITE

CRUS

DFE

DIOC

ECEC

EEE

EFP

ENIC

ESPA

EU-SILC

ISEI

NEET

ODM

OFFT
Association européenne de libre-échange

Adult Literacy and Life Skills Survey

(Enquête sur la littérarité et les compétences essentielles des adultes)

Commission fédérale des étrangers

Commission fédérale pour les questions de migration

Commission fédérale contre le racisme

Classification internationale type de l'éducation

Conférence des recteurs des universités suisses

Département fédéral de l'économie

OECD Database on Immigrants and Expatriates in OECD Countries

(Base de données sur les immigrés dans les pays de l'OCDE)

Early Childhood Education and Care

(Éducation et accueil des jeunes enfants)

Espace économique européen

Éducation et formation professionnelle

European Network of National Information Centers on Academic Recognition and Mobility

(Réseau européen commun des centres nationaux d'information sur la mobilité et la reconnaissance universitaire)

Enquête suisse sur la population active

EU Survey of Income and Living Conditions

(Enquête de l'UE sur les revenus et les conditions de vie)

International Socio-Economic Index of Occupational Status

(Indice socioéconomique international du statut professionnel)

Neither in Employment nor in Education or Training

(Ni en emploi, ni scolarisé, ni en formation)

Office fédéral des migrations

Office fédéral de la formation professionnelle et de la technologie 
OFIAMT Office fédéral de l'industrie des arts et métiers et du travail

OIE Ordonnance du 24 octobre 2007 sur l'intégration des étrangers

ONG Organisation non-gouvernementale

ORP Offices régionaux de placement

OSAR Organisation suisse d'aide aux réfugiés

PAMT Politiques actives du marché du travail

PISA OECD Programme for International Student Assessment

(Programme international pour le suivi des acquis des élèves)

PSM Panel suisse de ménages

SECO Secrétariat d'Etat à l'économie

SESAM Protection sociale et marché du travail (Enquête)

SPE

Service public de l'emploi

SYMIC Système d'information central sur la migration, SYMIC

TREE Transition Ecole-Emploi

UNHCR United Nations High Commissioner for Refugees

(Haut Commissariat des Nations unies pour les réfugiés) 
ANNEXE 1

Graphique 1 : Distribution du salaire horaire brut des employés à plein temps en Suisse, 15-64 ans et ayant terminé leur scolarité, 2009

(salaire horaire médian de l'ensemble de la population active)

口Suisses $\quad$ Immigrés nés dans un pays à revenus élevés $\quad$ Immigrés nés dans un pays à bas revenus

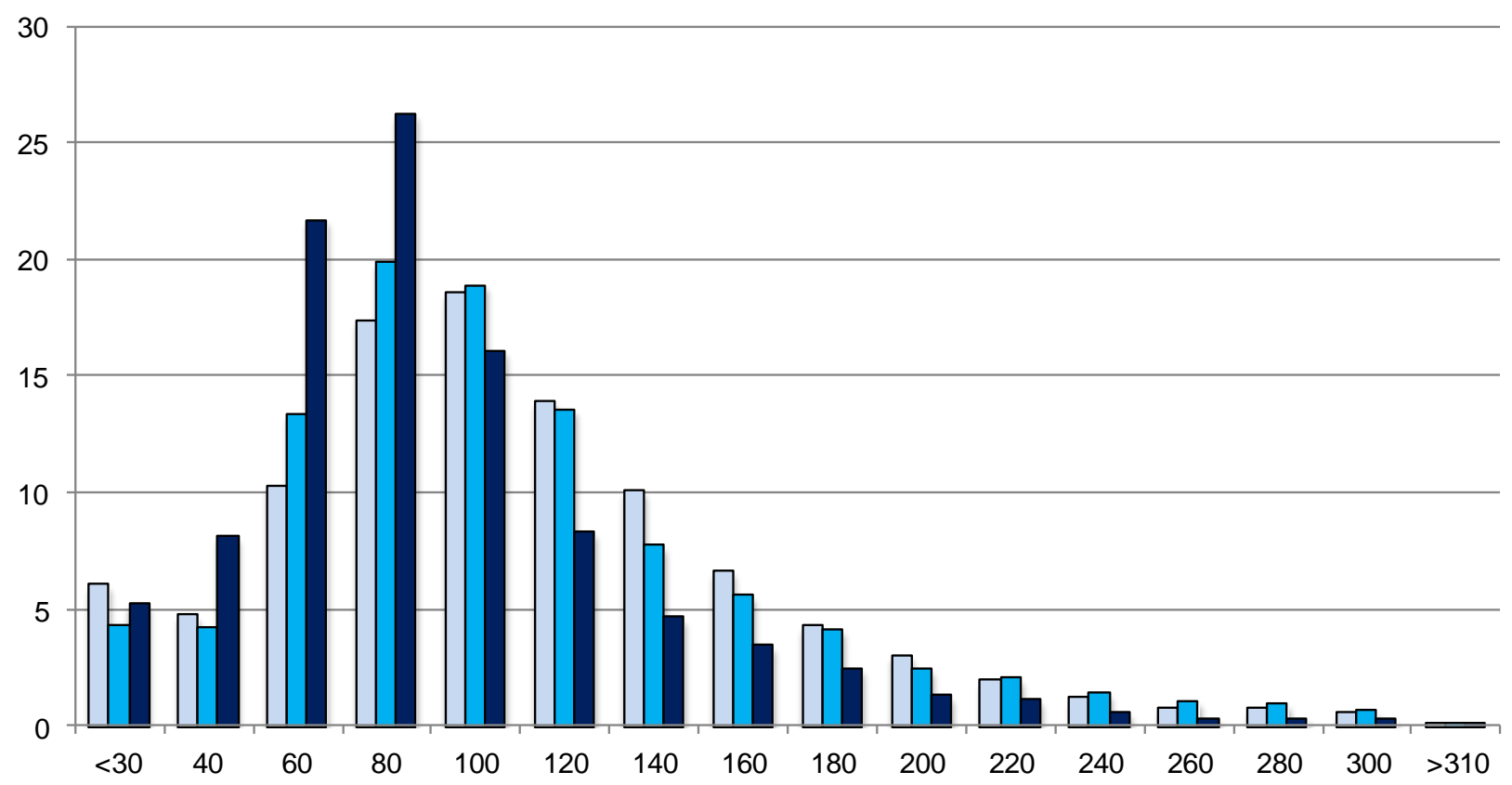

Note : Les chiffres de l'axe des abscisses désignent le milieu de chaque intervalle respectif (par exemple, 100=90\%-110\% du salaire horaire médian).

Source : Données provenant de l'Enquête suisse sur la population active (Office fédéral de la statistique) liées aux données administratives sur les salaires (SESAM). 
Graphique 2 : Principaux pays d'origine de la population migrante actuelle, 2008

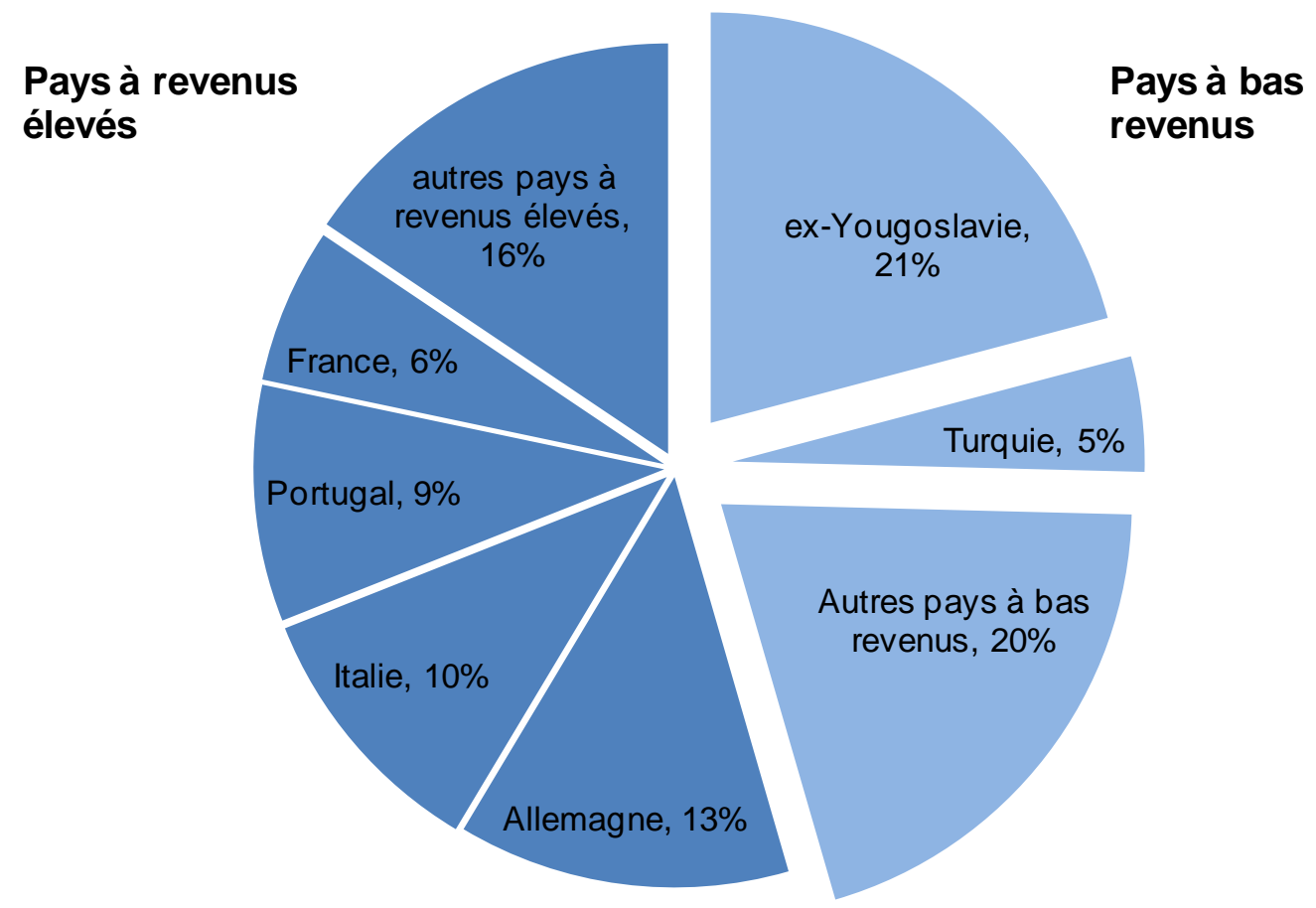

\begin{tabular}{|lrr|}
\hline Pays d'origine & $\%$ \\
\hline \hline ex-Yougoslavie & Kosovo 7\% & $21 \%$ \\
& Serbie 4\% & \\
& Macédoine 4\% & \\
& Bosnie-Herzégovine 3\% \\
& Croatie 2\% & \\
& Slovénie 0\% & \\
& Monténégro 0\% & \\
Allemagne & & $13 \%$ \\
Italie & & $10 \%$ \\
Portugal & & $9 \%$ \\
France & & $6 \%$ \\
Turquie & & $5 \%$ \\
Espagne & & $3 \%$ \\
Royaume-Uni & & $2 \%$ \\
Autriche & & $2 \%$ \\
États-Unis & & $1 \%$ \\
Brésil & $1 \%$ \\
Sri Lanka & & $1 \%$ \\
Inde & & $1 \%$ \\
Pays-Bas & & $1 \%$ \\
\hline
\end{tabular}

Source : Enquête suisse sur la population active (Office fédéral de la statistique). 
Graphique 3 : Population immigrée par régions et par principaux groupes migratoires, 2009

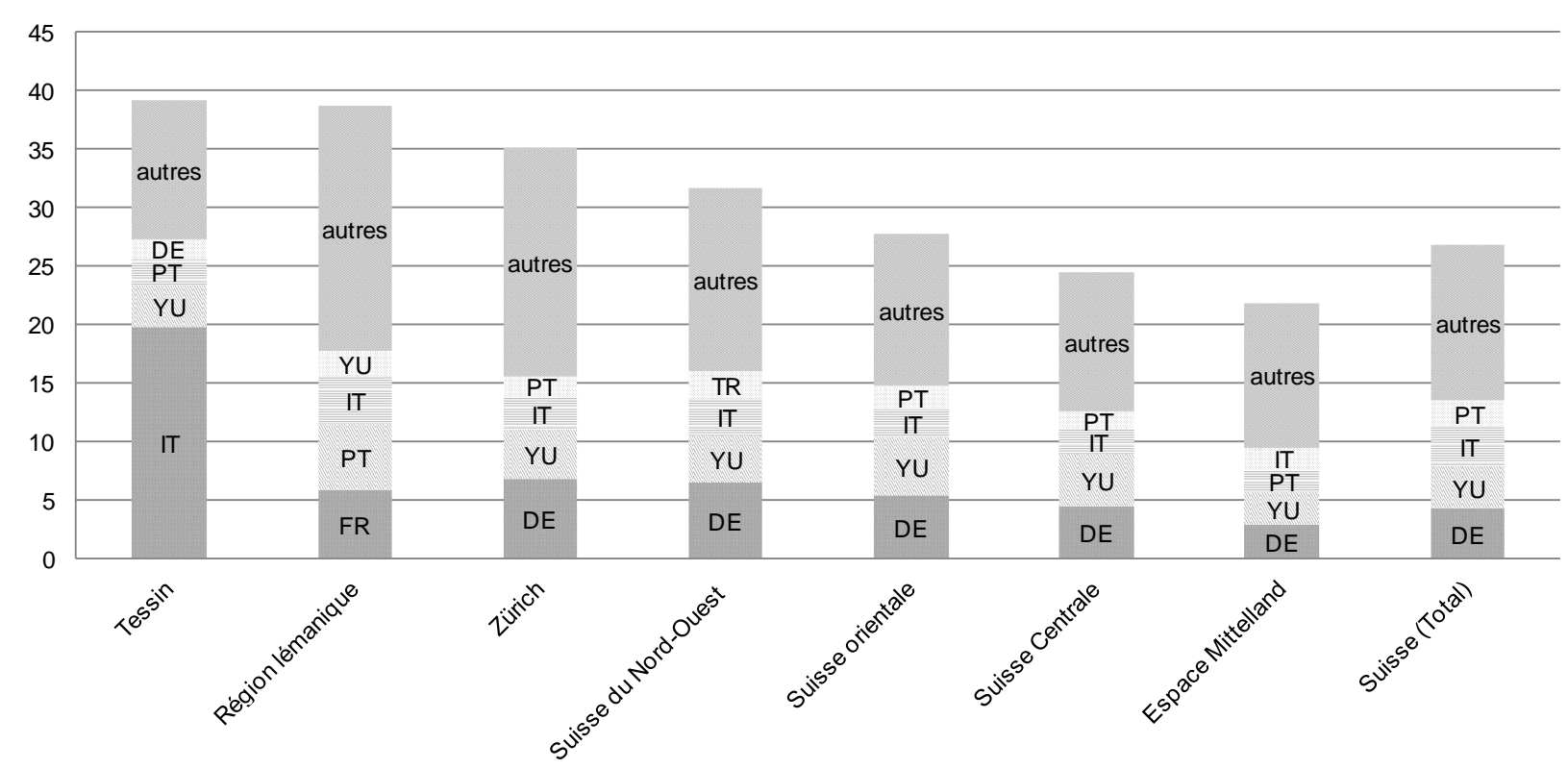

Source : Enquête suisse sur la population active (Office fédéral de la statistique).

Note : Le classement des régions est le suivant :

\section{Regions Cantons}

Région lémanique Vaud, Valais, Genève

Espace Mittelland $\quad$ Berne, Fribourg, Soleure, Neuchâtel, Jura

Suisse du Nord-

Ouest

Bâle-Ville, Bâle-Campagne, Aargau

Zürich Zürich

Glaris, Schaffhouse, Appenzell Rhodes-Extérieures, Appenzell Rhodes-Intérieures, Saint-Gall, Grisons, Thurgovie

Suisse orientale

Suisse Centrale Lucerne, Uri, Schwyz, Obwalden, Nidwalden, Zug

Tessin Tessin 


\section{Graphique 4 : Répartition des immigrés d'ex-Yougoslavie par année d'arrivée et région de résidence, 15-64 ans, 2009}

: Arrivés avant $1991 \quad$ Arrivés en 1992 et après

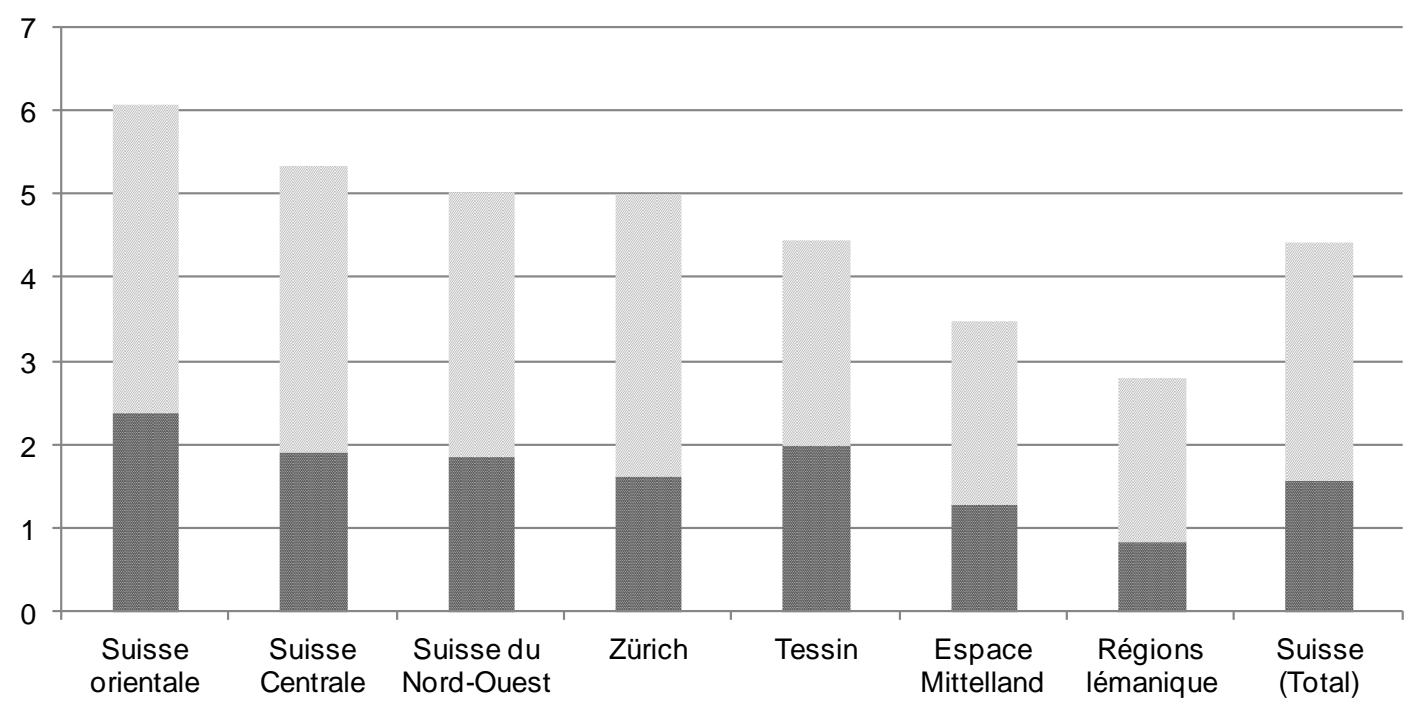

Note: Pour la classification des régions, voir la note du graphique 3, en annexe. 1. Sans la Slovénie.

Source : Enquête suisse sur la population active (Office fédéral de la statistique).

\section{Graphique 5 : Emploi des personnes nées à l'étranger, dans le service public pour quelques pays} membres de l'OCDE, 15-64 ans, 2006/2007

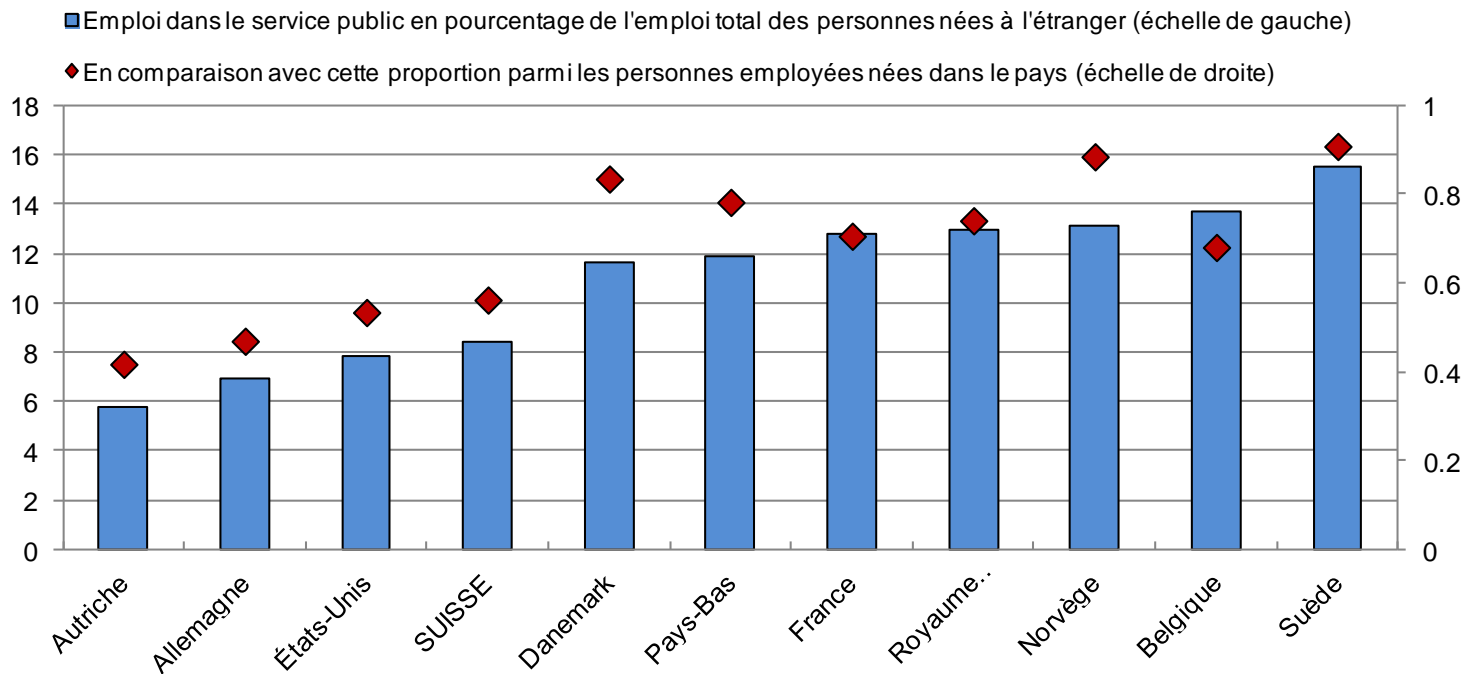

Source : Enquête européenne sur la population active. 
DELSA/ELSA/WD/SEM(2012)2

Tableau 1 : Salaires des immigrés récents selon le pays de naissance et le genre, 15-64 ans, 2008

\begin{tabular}{|l|r|cc|}
\hline & Total & $\begin{array}{c}\text { Salaire horaire brut médian } \\
\text { Hommes }\end{array}$ & Femmes \\
\hline Total des nouvelles arrivées & 38 & 39 & 34 \\
provenant de l'UE-27 & 41 & 44 & 39 \\
- d'Allemagne & 32 & 32 & 30 \\
- d'ltalie & 26 & 28 & 24 \\
- du Portugal & 45 & 49 & 34 \\
- Autres & & & 32 \\
& 36 & 38 & 32 \\
\hline Tous les immigrés récents & 35 & 43 & \\
Tous les immigrés & 40 & & \\
Personnes nées dans le pays &
\end{tabular}

Note : Prise en considération, seulement, des employés à temps plein qui ont travaillé au cours des douze derniers mois "sans interruption longue".

Source : Enquête suisse sur la population active (Office fédéral de la statistique), SESAM.

\begin{tabular}{|c|c|c|c|c|c|c|}
\hline & \multicolumn{3}{|c|}{ Hommes } & \multicolumn{3}{|c|}{ Femmes } \\
\hline & $\begin{array}{l}\text { (1) différence } \\
\text { brute }\end{array}$ & $\begin{array}{l}\text { (2) en tenant compte de } \\
\text { l'expérience, l'éducation } \\
\text { et la région }\end{array}$ & $\begin{array}{l}\text { (3) (2) plus la profession, } \\
\text { région }\end{array}$ & $\begin{array}{l}\text { (1) différence } \\
\text { brute }\end{array}$ & $\begin{array}{l}\text { en tenant compte } \\
\text { de l'expérience, } \\
\text { l'éducation et la } \\
\text { région }\end{array}$ & $\begin{array}{l}\text { (3) (2) plus la profession, } \\
\text { région }\end{array}$ \\
\hline $\begin{array}{l}\text { Total des nouvelles arrivées } \\
\text { provenant de l'UE-27 }\end{array}$ & & & & & & \\
\hline - d' Allemagne & $5.7^{\star \star \star}$ & $4.3^{\star \star}$ & $3.7^{\star \star}$ & $8.3^{\star \star \star}$ & $5.0^{\star \star \star}$ & $4.5^{\star \star \star}$ \\
\hline - d'Italie & $-7.8^{* \star}$ & 2.4 & 2.2 & 0.1 & 2.0 & 1.7 \\
\hline - du Portugal & $-18.6^{* * *}$ & 1.3 & $3.6^{\star *}$ & $-8.6^{\star \star \star}$ & 0 & 2.1 \\
\hline - Autre & $29.6^{\star \star \star}$ & $22.1^{\star * \star}$ & $21.2^{* * *}$ & $6.5^{\star \star \star}$ & 2.6 & 2.9 \\
\hline EU-27 $>5$ ans & $2.9^{\star \star \star}$ & $2.4^{\star \star}$ & $2.8^{\star \star}$ & $3.7^{* * \star}$ & 0.2 & 1.0 \\
\hline $\begin{array}{l}\text { Venant des pays non membres de } \\
\text { l'U-E27 (immigrés récents) } \\
\text { Venant des pays non membre de }\end{array}$ & 3.3 & $8.2^{\star * *}$ & $9.8^{* * *}$ & -1.0 & 0.1 & 1.6 \\
\hline l'EU à 27 (>5 ans) & $-10.7^{\star \star *}$ & $-5.6^{\star \star}$ & -3.0 & $-6.1^{\star * *}$ & $-5.4^{\star \star *}$ & $-3.8^{\star \star \star}$ \\
\hline constante & 48.0 & 22.5 & 17.9 & 32.9 & 18.0 & 11.7 \\
\hline
\end{tabular}

Note: La variable dépendante est le salaire horaire, qui a été déduit de la rémunération totale brute au cours des 12 derniers mois, en utilisant la moyenne des heures de travail, par an, en Suisse (1926 heures selon l'Office fédéral de la statistique). Seulement les personnes qui ont déclaré avoir été employées "sans longue interruption" pendant cette période sont prises en compte. Les immigrés nés aux États-Unis, au Canada, au Japon, en Corée, en Australie ou en Nouvelle-Zélande ainsi que les personnes interrogées gagnant 0 ou plus de $1000 \mathrm{CHF}$ par heure ont été exclues. Le groupe de référence est celui des personnes nées dans le pays. ${ }^{\star *},{ }^{* *},{ }^{* * *}$ indiquent un seuil de significativité de $1 \%, 5 \%$, et $10 \%$ respectivement.

Source : Enquête suisse sur la population active (Office fédéral de la statistique), SESAM. 
Tableau 2 : Résultat sur le marché du travail des personnes ayant un niveau d'études élevé dans quelques pays à revenus élevés de I'OCDE, 15-64 ans, 2008/2009

\begin{tabular}{|c|c|c|c|c|c|c|}
\hline \multicolumn{7}{|c|}{ Pourcentage des personnes agées de 15 à 64 ans... } \\
\hline & & $\begin{array}{c}\text { Emploi } \\
\text { hautement } \\
\text { qualifié }\end{array}$ & $\begin{array}{l}\text { Emploi } \\
\text { moyennement } \\
\text { qualifié }\end{array}$ & $\begin{array}{l}\text { Emploi peu } \\
\text { qualifié }\end{array}$ & Chômage & Inactif \\
\hline \multicolumn{7}{|l|}{ Autriche } \\
\hline & nés dans le pays & 70 & 17 & (1) & (2) & 10 \\
\hline & nés à l'étranger & 55 & 16 & (5) & (4) & 19 \\
\hline & nés à l'étranger, pays à revenus élevés & 65 & 14 & $\ldots$ & $\ldots$ & 17 \\
\hline & nés à l'étranger, pays à bas revenus & 40 & 20 & (11) & $\ldots$ & 23 \\
\hline \multicolumn{7}{|l|}{ Belgique } \\
\hline & nés dans le pays & 65 & 18 & 1 & 3 & 13 \\
\hline & nés à l'étranger & 51 & 19 & 3 & 8 & 19 \\
\hline & nés à l'étranger, pays à revenus élevés & 60 & 17 & $\ldots$ & 5 & 17 \\
\hline & nés à l'étranger, pays à bas revenus & 41 & 20 & 5 & 12 & 21 \\
\hline \multicolumn{7}{|c|}{ 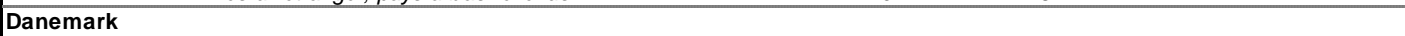 } \\
\hline & nés dans le pays & 76 & 11 & (1) & 3 & 9 \\
\hline & nés à l'étranger & 57 & 16 & $\ldots$ & (8) & 14 \\
\hline & nés à l'étranger, pays à revenus élevés & 68 & (12) & $\cdots$ & $\ldots$ & (12) \\
\hline & nés à l'étranger, pays à bas revenus & 39 & (25) & $\cdots$ & $\cdots$ & $\ldots$ \\
\hline \multicolumn{7}{|l|}{ France } \\
\hline & nés dans le pays & 64 & 16 & 1 & 4 & 14 \\
\hline & nés à l'étranger & 51 & 15 & 3 & 8 & 22 \\
\hline & nés à l'étranger, pays à revenus élevés & 59 & 11 & $\ldots$ & (7) & 21 \\
\hline & nés à l'étranger, pays à bas revenus & 49 & 17 & 4 & 8 & 22 \\
\hline \multicolumn{7}{|c|}{ 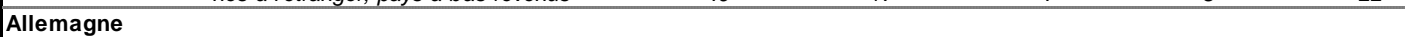 } \\
\hline & nés dans le pays & 71 & 17 & 1 & 3 & 14 \\
\hline & nés à l'étranger & 54 & 20 & 5 & 7 & 14 \\
\hline & nés à l'étranger, pays à revenus élevés & $\ldots$ & $\ldots$ & $\ldots$ & $\ldots$ & $\ldots$ \\
\hline & nés à l'étranger, pays à bas revenus & $\ldots$ & $\ldots$ & $\ldots$ & $\ldots$ & $\ldots$ \\
\hline \multicolumn{7}{|l|}{ Pays-Bas } \\
\hline & nés dans le pays & 77 & 10 & 1 & 2 & 10 \\
\hline & nés à l'étranger & 58 & 15 & (3) & 4 & 19 \\
\hline & nés à l'étranger, pays à revenus élevés & 69 & 13 & $\ldots$ & $\ldots$ & 12 \\
\hline & nés à l'étranger, pays à bas revenus & 52 & 16 & (3) & (5) & 23 \\
\hline \multicolumn{7}{|c|}{ 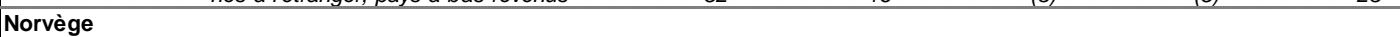 } \\
\hline & nés dans le pays & 80 & 9 & $\ldots$ & 1 & 8 \\
\hline & nés à l'étranger & 61 & 18 & $\ldots$ & $\ldots$ & 14 \\
\hline & nés à l'étranger, pays à revenus élevés & 73 & $\ldots$ & $\ldots$ & $\ldots$ & $\ldots$ \\
\hline & nés à l'étranger, pays à bas revenus & 49 & 25 & $\ldots$ & $\ldots$ & 17 \\
\hline \multicolumn{7}{|c|}{ 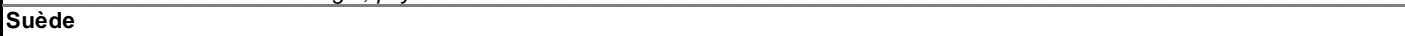 } \\
\hline & nés dans le pays & 79 & 9 & 1 & 3 & 8 \\
\hline & nés à l'étranger & 52 & 20 & 4 & 9 & 15 \\
\hline & nés à l'étranger, pays à revenus élevés & 67 & 16 & $\ldots$ & (5) & 10 \\
\hline & nés à l'étranger, pays à bas revenus & 41 & 22 & 6 & 13 & 18 \\
\hline \multicolumn{7}{|l|}{ Royaume-Uni } \\
\hline & nés dans le pays & 65 & 18 & 2 & 4 & 12 \\
\hline & nés à l'étranger & 58 & 19 & 3 & 5 & 15 \\
\hline & nés à l'étranger, pays à revenus élevés & 65 & 15 & 3 & 5 & 12 \\
\hline & nés à l'étranger, pays à bas revenus & 55 & 21 & 2 & 6 & 17 \\
\hline \multicolumn{7}{|c|}{ 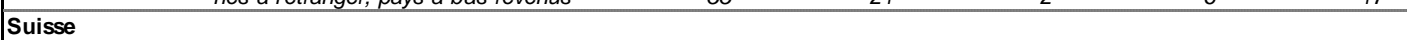 } \\
\hline & nés dans le pays & 72 & 18 & 1 & 2 & 7 \\
\hline & nés à l'étranger & 69 & 14 & (1) & 4 & 12 \\
\hline & nés à l'étranger, pays à revenus élevés & 76 & 12 & $\ldots$ & 3 & 10 \\
\hline & nés à l'étranger, pays à bas revenus & 53 & 18 & (3) & 7 & 18 \\
\hline \multicolumn{7}{|l|}{ États-Unis } \\
\hline & nés dans le pays & 53 & 26 & 3 & 3 & 15 \\
\hline & nés à l'étranger & 49 & 25 & 4 & 4 & 18 \\
\hline & nés à l'étranger, pays à revenus élevés & 53 & 23 & 3 & 3 & 18 \\
\hline & nés à l'étranger, pays à bas revenus & 48 & 26 & 5 & 4 & 17 \\
\hline \multicolumn{7}{|l|}{ Moyenne OCDE ${ }^{1}$} \\
\hline & nés dans le pays & 72 & 14 & 1 & 3 & 10 \\
\hline & nés à l'étranger & 52 & 16 & 3 & 6 & 15 \\
\hline & nés à l'étranger, pays à revenus élevés & 60 & 12 & 3 & 4 & 12 \\
\hline & nés à l'étranger, pays à bas revenus & 42 & 18 & 3 & 8 & 18 \\
\hline
\end{tabular}

Note : 1. La moyenne du groupe OCDE fait référence à la moyenne des pays inclus dans le tableau et pour lesquels des données sont disponibles.

Source : Enquête européenne sur la population active 2008-2009 et Current Population Survey Survey, March Supplement 2009 pour les États-Unis. 
Tableau 3 : Taux d'emploi et taux de chômage des immigrés nés en ex-Yougoslavie, selon le genre et l'année d'arrivée, 15-64 ans, 2009

\begin{tabular}{lrrrrrr} 
& \multicolumn{3}{c}{ Taux d'emploi (\%) } & \multicolumn{4}{c}{ Taux de chômage (\%) } \\
\cline { 2 - 7 } Arrivée & Total & Avant 1992 & 1992 et après & Total & Avant 1992 & 1992 et après \\
\hline Hommes & 73.1 & 75.8 & 71.3 & 8.1 & 5.8 & 9.7 \\
\hline Femmes & 55.3 & 59.1 & 53.6 & 10.7 & 8.1 & 11.9 \\
\hline Total & 64.3 & 68.7 & 61.9 & 9.2 & 6.7 & 10.7
\end{tabular}

Source : Enquête suisse sur la population active (Office fédéral de la statistique). 
DELSA/ELSA/WD/SEM(2012)2

ANNEXE 2.

\section{ZUSAMMENFASSUNG}

Die Schweiz ist innerhalb der OECD eines der Länder mit dem höchsten Immigrantenanteil - $27 \%$ der Personen im erwerbsfähigen Alter sind im Ausland geboren - und das Thema Einwanderung nimmt sowohl in der politischen Agenda als auch in der öffentlichen Diskussion einen wichtigen Platz ein. In Anbetracht der zahlreichen Debatten, die das Thema im Land auslöst, könnte man versucht sein zu glauben, die Immigranten seien in der Schweiz weniger gut integriert als in anderen Ländern.

Die Fakten zeigen jedoch, dass die Integration in der Schweiz im Grossen und Ganzen relativ gut funktioniert. Auf dem Arbeitsmarkt ist die Lage der zugewanderten Bevölkerung insgesamt bedeutend besser als in anderen Ländern,. Sowohl bei den Männern als auch bei den Frauen ist die Beschäftigungsquote der Immigranten höher als in den anderen Ländern der OECD.

Diese guten Ergebnisse lassen sich hauptsächlich durch die insgesamt gute Lage auf dem Schweizer Arbeitsmarkt erklären, aber auch durch eine spezielle Konstellation bei den Herkunftsländern. Der Grossteil der Migranten (mehr als $60 \%$ ) stammen aus OECD-Ländern mit hohen Einkommensniveau, mehr als die Hälfte davon aus den Nachbarländern, in denen eine der Schweizer Landessprachen gesprochen wird. Die anderen Immigranten stammen hauptsächlich aus den Ländern Ex-Jugoslawiens und der Türkei.

Im Laufe der letzten Jahre sind als Folge der schrittweisen Einführung des freien Personenverkehrs mit den Mitgliedländern der Europäischen Union aussergewöhnlich viele Zuwanderer in die Schweiz gekommen. Ungefähr $5 \%$ der Wohnbevölkerung sind Menschen, die erst seit kurzem, d.h. seit weniger als fünf Jahren, in der Schweiz wohnen. Diese neuen Einwanderer stammen mehrheitlich aus Nachbarländern, insbesondere aus Deutschland. Alle konventionellen Indikatoren zeigen, dass sie sich gut auf dem Arbeitsmarkt integrieren.

Trotz diesem im Ganzen positiven Bild ist die Lage für gewisse Einwanderergruppen schwieriger, beispielsweise für Frauen mit kleinen Kindern. Es wird wenig unternommen, um die Integration der eingewanderten Frauen zu fördern, die häufig nicht Zugang zu allen Massnahmen der aktiven Arbeitsmarktpolitik haben. Gewisse Elemente scheinen ausserdem darauf hinzuweisen, dass die Beschäftigungsquote dieser Bevölkerungsgruppe seit einigen Jahren sinkt.

Eine weitere Gruppe verzeichnet schlechte Beschäftigungsquoten, und dies auch im internationalen Vergleich: Personen, die vor kurzem aus humanitären Gründen eingewandert sind, scheinen bei der Integration in den schweizerischen Arbeitsmarkt mehr Mühe zu bekunden als frühere Kohorten humanitärer Migranten. Im Gegensatz zu anderen Ländern gibt es in der Schweiz noch kein allgemeines Programm für die Integration dieser Kategorie von Neuankömmlingen. Dies kann zum Teil deren schlechte Leistungen erklären. Angesichts der ermutigenden Erfahrungen, die andere OECD-Länder mit strukturierten und auf den Arbeitsmarkt ausgerichteten Integrationsprogrammen gemacht haben, wäre es wünschenswert, dass die Schweiz ernsthaft erwägt, ebenfalls solche Programme umzusetzen.

Die allgemein hohe Beschäftigungsquote der Immigranten in der Schweiz ist auch mit einer häufigen Überqualifizierung derjenigen Migranten verbunden, die in Nicht-OECD-Ländern ein Diplom erworben 
haben. Qualifikationen, die im Ausland erworben wurden, werden auf dem schweizerischen Arbeitsmarkt ungenügend anerkannt, und es gibt nur wenige Brücken- bzw. Passerelle-Kurse. Im Gegensatz zu anderen Ländern der OECD gibt es in der Schweiz auch nur wenige Patenschaftsprogramme oder ähnliche Initiativen die es den Immigranten ermöglichen würden, die unabdingbaren Kontakte zu in der Schweiz geborenen Personen und zu Arbeitgebern zu knüpfen und sich gleichzeitig mit den Abläufen des Arbeitsmarkts vertraut zu machen; zwei Aspekte, die entscheidend sind, um Zugang zu hochqualifizierten Stellen zu erhalten. Solche Massnahmen sollten stärker verbreitet werden, in Zusammenarbeit mit den Arbeitgebern.

In der Integrationspolitik zeigt sich klar der föderalistische Charakter des Landes. Verschiedene Massnahmen wurden auf lokaler und kantonaler Ebene entwickelt, um die Integration zu fördern. Die Anwendung des Subsidiaritätsprinzips hat einerseits dazu geführt, dass punktuelle und flexible Massnahmen zugunsten vieler Immigranten getroffen wurden, sie hat jedoch gleichzeitig die Entwicklung einer Integrationspolitik des Bundes verzögert. Infolgedessen ist die allgemeine Architektur in Bezug auf die Integration weiterhin unterentwickelt, trotz einer deutlichen Verbesserung in den letzten zehn Jahren. Die Integrationspolitik des Bundes ist alles in allem bescheiden, wenn man sie mit der anderer OECDLänder vergleicht, von denen die meisten einen niedrigeren Einwandereranteil haben als die Schweiz. Mit Ausnahme einiger Instrumente wie beispielsweise dem vom Bundesamt für Migration finanzierten sprachlichen Grundkurs sind in der Schweiz nur wenige Integrationsmassnahmen spezifisch auf Immigranten ausgerichtet. Man geht allgemein davon aus, dass die Immigranten von den allen zugänglichen Massnahmen profitieren werden, anstatt spezifisch auf Immigranten ausgerichtete Politiken zu gestalten. Um die Mängel des aktuellen Systems etwas zu mildern, haben Bund, Kantone und lokale Behörden kürzlich einige Vorschläge formuliert, wie der allgemeine Rahmen für die Integration verbessert werden könnte. Sie haben sich zudem verpflichtet, die Mittel für die Integration aufzustocken.

Auf kantonaler Ebene sind die Massnahmen bei der Integrationshilfe sehr unterschiedlich, was teilweise auf die Unterschiede in der Grösse und der Zusammensetzung der zugewanderten Bevölkerung zurück zu führen ist. Wohl haben zahlreiche Kantone in den letzten Jahren die Integrationsmassnahmen verstärkt, dies jedoch häufig nur in kleinem Umfang und im Rahmen von Beispielprojekten, was eine Evaluation deren Wirksamkeit schwierig macht. Die Bundesbehörden sollten Minimalnormen festlegen, damit alle Immigranten unabhängig vom Wohnkanton von den von ihnen benötigten Massnahmen profitieren können.

Für die Immigranten ist es schwierig, das Schweizer Bürgerrecht zu erwerben. Die erforderliche Aufenthaltsdauer ist in der Tat besonders lang - 12 Jahre für ein normales Verfahren, die längste Frist innerhalb der OECD - und das Verfahren für den Erwerb läuft auf drei Ebenen: Der Kandidat muss sowohl den Anforderungen des Bundes als auch denjenigen des Kantons und der Gemeinde genügen. Eine Reform der Gesetzgebung ist im Gang, sie sollte einige der wichtigsten Lücken im Bürgerrechtsgesetz schliessen und die Mobilität der Migranten innerhalb der Schweiz verbessern. Forschungsergebnisse deuten darauf hin, dass dies eine starke Dynamisierung der Integration benachteiligter Immigrantengruppen bewirken könnte.

Insgesamt erzielen die Kinder von Immigranten im internationalen Vergleich gute Resultate auf dem Arbeitsmarkt, was zum Teil auf die allgemein guten Bedingungen auf dem Arbeitsmarkt zurück zu führen ist. Andere Faktoren spielen ebenfalls eine Rolle, wie beispielsweise die starke Verbreitung der Berufslehre, die sich für Immigrantenkinder im Übergang von der Schule in die Arbeitswelt besonders positiv auszuwirken scheint. Es wurden auch einige innovative Programme lanciert, um niedrigqualifizierte Jugendliche (von denen ein Grossteil Immigrantenkinder sind) auf die Berufslehre vorzubereiten. Diese Programme scheinen eine positive Wirkung gehabt zu haben. 
Immigrantenkinder, deren Eltern ein tiefes Bildungsniveau haben, erzielen in der Schule schlechte Leistungen. Gegenwärtig treten diese Immigrantenkinder in steigender Zahl in den Arbeitsmarkt ein. Es scheint, dass diese weniger befriedigenden Leistungen zumindest teilweise darauf zurück zu führen sind, dass das Bildungssystem keine Betreuung ab einem genügend frühen Alter vorsieht. Die Einschulung der Kleinkinder ist in der Schweiz noch nicht sehr weit entwickelt. Es sollte dringend eine Priorität des staatlichen Handelns werden, den Immigrantenkindern ab dem entscheidenden Alter von drei oder vier Jahren einen angepassten und zielgerichteten Unterricht sowie Möglichkeiten zum Erlernen der Sprache anzubieten.

In einem Bereich weist die Schweiz einen klaren Rückstand auf andere OECD-Länder auf, und zwar im Kampf gegen Diskriminierung. Den Arbeitgebern ist das Problem, das ausserdem nicht öffentlich diskutiert wird, zu wenig bewusst. Studien haben jedoch gezeigt, dass Immigrantenkinder, insbesondere wenn die Eltern aus ExJugoslawien stammen, bei gleichen Qualifikationen bis zu fünf Mal mehr Bewerbungen schreiben müssen als Kinder ohne Migrationshintergrund, um zu einem Vorstellungsgespräch eingeladen zu werden. Es ist wichtig, die erforderlichen Massnahmen zu treffen, um dieser Ungleichbehandlung entgegenzuwirken.

Auch wenn die Schweiz im Grossen und Ganzen im internationalen Vergleich insgesamt gute Resultate erzielt bei der Integration der zugewanderten Bevölkerung in den Arbeitsmarkt, gibt es dennoch mehrere Anzeichen dafür, dass die Situation für gewisse Gruppen von Migranten divergiert. Einige benachteiligte Gruppen stehen in der Gefahr, auf der Strecke zu bleiben. Es ist daher wichtig, dass sofort gehandelt wird, solange die Resultate gesamthaft noch positiv sind. Dies ist erkannt worden, und die Integrationsbemühungen werden auf allen drei staatlichen Ebenen verstärkt. In vielerlei Hinsicht bleibt die Integrationspolitik der Schweiz jedoch hinter dem zurück, was in anderen OECD-Ländern geleistet wird. Es sollten Massnahmen ins Auge gefasst werden, um diese Situation zu verbessern und zu erreichen, dass die Resultate in Zukunft für alle Gruppen von Immigranten zufriedenstellend sind.

\section{Zusammenfassung der wichtigsten Politikempfehlungen}

\section{A) Den übergeordneten Rahmen für die Integration stärken}

- Gemeinsame Minimalnormen für Integrationsmassnahmen entwickeln, die für alle Kantone gelten.

- Den Austausch von guten Praktiken zwischen Kantonen und Gemeinden erleichtern.

- Sicherstellen, dass alle Immigranten, die Integrationshilfe benötigen, diese auch erhalten, unabhängig vom Bewilligungstypus und der Art und Höhe von Leistungen, die sie beziehen. Dies ist besonders für Immigrantinnen wichtig.

- Allen Immigranten, die eine sprachliche Förderung benötigen, entsprechende Möglichkeiten anbieten, unter Berücksichtigung ihrer Kenntnisse und Qualifikationen.

- Den Zugang zum Schweizer Bürgerrecht vereinfachen, indem insbesondere die Anforderungen der Kantone und Gemeinden bezüglich Aufenthaltsdauer reduziert werden, um Migranten die geographische Mobilität zu erleichtern.

- Auf die Vorteile aufmerksam machen, welche der Erwerb des Schweizer Bürgerrechts für eine bessere Integration der Immigranten und ihrer Kinder mit sich bringt.

B) Bei Migranten, die aus humanitären Gründen eingewandert sind, eine schnelle Integration in den Arbeitsmarkt fördern

- Stärkere Anreize für die Kantone, um Migranten, die aus humanitären Gründen eingewandert sind, innerhalb der ersten fünf Aufenthaltsjahre schnell in den Arbeitsmarkt zu integrieren.

- Umsetzen eines strukturierten Integrationsprogramms zugunsten aller Migranten, die vor kurzem aus humanitären Gründen eingewandert sind (Asylbewerber, deren Antrag angenommen wurde oder die vorläufig aufgenommen sind), je nach den Bedürfnissen der Einzelnen. Der Schwerpunkt sollte klar auf der Integration in den Arbeitsmarkt liegen.

- $\quad$ Eine bessere Information der Arbeitgeber über den Zugang zum Arbeitsmarkt von Personen, die vorläufig aufgenommen sind. 


\section{Zusammenfassung der wichtigsten Politikempfehlungen (Fortsetzung)}

\section{C) Die Fähigkeiten der Migranten besser nutzen}

- $\quad$ Sicherstellen, dass der gegenwärtige Schwerpunkt aktuell auf wenig qualifizierter Arbeit bei Migranten, die aus humanitären Gründen eingewandert sind, nicht ein Hindernis darstellt für eine bestmögliche Nutzung ihrer Fähigkeiten.

- Unter den Immigranten die Angebote für Evaluation und Anerkennung von im Ausland erworbenen Qualifikationen besser bekannt machen. Die Vorteile einer solchen Anerkennung stärker aufzeigen.

- Brückenkurse bzw. Passerellen-Angebote und andere Unterstützungsprogramme einrichten, um Immigranten mit im Ausland erworbenen Diplomen bei der Suche nach einer besser qualifizierten Stelle zu helfen.

- Eine auf Migranten ausgerichtete behutsame Ausdehnung von zeitlich befristeten Lohnsubventionen in Erwägung ziehen.

\section{D) Die Anstrengungen für eine rasche Integration der Immigrantenkinder verstärken}

- Die vorschulische Erziehung ausbauen und besonders darauf achten, dass Kinder von Immigranten aus benachteiligten Verhältnissen ab dem Alter von 3 oder 4 Jahren daran teilnehmen.

- Die sprachliche Förderung der Immigrantenkinder, verstärken, insbesondere im vorschulischen Bereich.

- Untersuchen, warum bei Immigrantenkindern der Prozentsatz der Lehrabschlüsse offenbar niedrig ist, und Massnahmen treffen, um dies zu ändern.

\section{E) Einen breit abgestützten Rahmen für den Kampf gegen Diskriminierung schaffen}

- Illegalisierung von Diskriminierung aufgrund der Nationalität in Personalauswahl und Einstellungsverfahren.

- Arbeitgeber und die Gesellschaft im Allgemeinen vermehrt für das Problem der Diskriminierung sensibilisieren.

- Entschiedenere Massnahmen für den Kampf gegen Diskriminierung in Erwägung ziehen. 


\section{BEURTEILUNG UND EMPFEHLUNGEN}

Die Schweiz weist einen hohen Anteil an Immigranten auf, und die Gesamtbilanz hinsichtlich der Integration in den Arbeitsmarkt ist positiv.

Dies ist teilweise darauf zurïck zu führen, dass in der Schweiz nahezu Vollbeschäftigung herrscht und die meisten Immigranten aus Nachbarländern stammen.
Ungefähr $27 \%$ der in der Schweiz wohnhaften Personen sind im Ausland geboren. Das ist, neben Luxemburg und Australien, einer der höchsten Anteile der OECD-Zone. Im internationalen Vergleich sind die zentralen Indikatoren für die Integration der Immigranten in den Arbeitsmarkt insgesamt durchaus positiv. Die Schweiz ist innerhalb der OECD eines der Länder, in dem Immigranten beider Geschlechter die höchsten Beschäftigungsquoten aufweisen. Dies gilt ebenso für die in der Schweiz geborenen Kinder eingewanderter Eltern. Dennoch, und trotz der allgemein tiefen Arbeitslosenquote im Land, ist die Arbeitslosigkeit gewisser Immigrantengruppen nicht unbedeutend.

Das im Vergleich zu anderen OECD-Ländern recht positive Bild der Integration in der Schweiz dürfte sich im Wesentlichen auf zwei Faktoren zurück führen lassen. Erstens ist die Lage auf dem schweizerischen Arbeitsmarkt gesamthaft gesehen gut. Zweitens stammten und stammen die meisten Immigranten in der Schweiz, im Gegensatz zu anderen OECD-Ländern, aus Hocheinkommensländern, insbesondere aus den Nachbarländern Deutschland und Italien, den beiden häufigsten Herkunftsländern. In der Gruppe der Herkunftsländer mit tiefem Einkommensniveau stellen Ex-Jugoslawien und seine Nachfolgestaaten fast die Hälfte aller Immigranten. $12 \%$ der Migranten stammen aus der Türkei. Gründe für die Einwanderung waren im Wesentlichen die Arbeitsmigration und der damit zusammen hängende Familiennachzug. Der Anteil an Migranten, die aus humanitären Gründen eingewandert sind (und die in allen Ländern oft besondere Schwierigkeiten haben bei der Integration in den Arbeitsmarkt) ist jedoch kleiner als in anderen Ländern. Allerdings ist auch diese Gruppe zahlenmässig nicht unbedeutend, da die Schweiz in den letzten zwei Jahrzehnten eines der Hauptzielländer für Asylbewerber innerhalb der OECD war. 
Die Schweiz verzeichnet einen

hohen Anteil vor kurzem

eingewanderter Personen aus der

EU. Diese Bevölkerungsgruppe hat

sich im Allgemeinen gut in den

Arbeitsmarkt integriert.
Seit 2002 sind in Folge der schrittweisen Einführung der Freizügigkeit mit der EU und ihren Mitgliedstaaten sehr viele Personen aus der Europäischen Union in die Schweiz eingewandert. Mehr als $5 \%$ der Bevölkerung des Landes sind in diesem Zeitraum eingewandert. Innerhalb dieser Gruppe ist die soziodemographische Zusammensetzung je nach Herkunftsland sehr unterschiedlich. Besonders viele Immigranten aus Portugal sind arbeitslos. Im Allgemeinen erzielen jedoch die neu zugewanderten Migranten aus der erweiterten EU sehr gute Resultate auf dem Arbeitsmarkt. Insbesondere entspricht ihre Arbeitsstelle im Durchschnitt eher ihrer formellen Qualifikation, als dies bei den Nichtzuwanderern der Fall ist. Dies legt die Vermutung nahe, dass ihre Kompetenzen in hohem Masse mit der Nachfrage nach Arbeitskräften übereinstimmen.

Es gibt gewisse Anzeichen einer

Immigrantinnen aus Ländern mit niedrigen Einkommensniveau, insbesondere neu eingewanderte Frauen, sind eine Gruppe, deren Verschlechterung der Beschäftigungssituation für Frauen aus Ländern mit niedrigen Einkommensniveau. Diese Entwicklung gilt es im Auge zu behalten.
Resultate bezüglich der Beschäftigung nicht nur unvorteilhaft sind, sondern sich in den letzten Jahren noch verschlechtert haben. Dies ist eine beunruhigende Entwicklung, die aufmerksam verfolgt werden muss. In der Tat erreichen die Integrationsbemühungen der Schweiz die Immigrantinnen oft nicht, da die Frauen häufig nicht im Arbeitsmarkt sind und von keinen Leistungen profitieren können. Für diese Gruppe könnte die kürzlich erfolgte Reduktion der Mitfinanzierung (über die Arbeitslosenversicherung) der aktiven arbeitsmarktlichen Massnahmen für Personen ohne Arbeitslosengeld zur Folge haben, dass das bereits eingeschränkte Angebot an Integrationsmassnahmen weiter eingeschränkt wird, wenn nicht die Sozialdienste diese Reduktion auffangen. Im Gegensatz zu Migranten, die aus humanitären Gründen eingewandert sind, können Migranten, die durch Familiennachzug in die Schweiz gekommen sind, bei ihrer Ankunft nicht von einer einheitlichen Integrationshilfe profitieren. Sie können jedoch eine gewisse Unterstützung in der Form einer sprachlichen Grundausbildung erhalten. In den anderen OECD-Ländern hingegen werden Massnahmen zunehmend auf alle neu Ankommenden ausgeweitet, und/oder die Tätigkeiten von Nichtregierungsorganisationen kompensieren den Mangel an allgemeinen Angeboten. Beide Ansätze sind in der Schweiz eher unbedeutend. Da eine bessere Integration der Immigrantinnen in den schweizerischen Arbeitsmarkt auch den Kindern dieser Frauen wichtige Impulse geben kann, sollte eine Ausdehnung der Integrationsmassnahmen auf diese Bevölkerungsgruppe dringend und ernsthaft in Betracht gezogen werden. 
Die schlechte Situation der

Migranten, die vor kurzem aus

humanitären Gründen

eingewandert sind, verlangt nach

besser strukturierten Massnahmen

für die Integration.

Die Anreize der Kantone für eine rasche Integration der aus humanitären Gründen eingewanderten Personen in den Arbeitsmarkt müssen verstärkt werden...
Personen, die vor kurzem aus humanitären Gründen eingewandert sind, erzielen heute auf dem Arbeitsmarkt viel weniger gute Resultate als früher, zudem schneiden sie auch im internationalen Vergleich schlecht ab. Es ist nicht ganz klar, welche Gründe zu dieser Situation geführt haben. Auf jeden Fall handelt es sich hier um eine Gruppe, die gezielte Integrationsmassnahmen benötigt. Andere OECD-Länder haben strukturierte Integrationsprogramme für diese Gruppe umgesetzt. Diesen Massnahmen, die bis zu drei Jahren dauern können, scheint ein gewisser Erfolg beschieden zu sein. In Ländern wie Dänemark oder Norwegen, die über strukturierte, auf die Integration in den Arbeitsmarkt ausgerichtete Programme verfügen, haben sich die Resultate der Personen, die vor kurzem aus humanitären Gründen eingewandert sind, verbessert. Dies deutet darauf hin, dass die Schweiz stark profitieren könnte, wenn sie sich an diesem Ansatz orientieren würde.

Für die Sozialhilfe ist zwar nicht der Bund zuständig, auf Ebene der Kantone wird sie jedoch während den ersten fünf Aufenthaltsjahren der Flüchtlinge vom Bund bezahlt. Für die auch zahlenmässig immer wichtigere Gruppe der vorläufig aufgenommenen Personen bietet der Bund sogar eine Finanzierung während der ersten sieben Jahre, bevor die Kantone /Gemeinden für diese Personen zuständig sind. Vorläufig aufgenommene Personen erhalten zudem weniger als die ordentliche Sozialhilfe, und diese Tatsache scheint manchmal mit einer Verminderung der Integrationsbemühungen für diese Gruppe einher zu gehen. Dies bringt mit sich, dass für die Kantone Anreize fehlen, um die aus humanitären Gründen eingewanderten Personen während der ersten fünf bis sieben Jahre in den Arbeitsmarkt zu integrieren. Wenn die Immigranten jedoch während dieser Zeit nicht richtig vorbereitet werden, werden sie beim Eintritt in den Arbeitsmarkt grosse Schwierigkeiten haben. Gemäss Analysen aus anderen OECD-Ländern ist ein schneller Eintritt in den Arbeitsmarkt jedoch ein entscheidender Faktor für eine langfristige Integration. Die Anreize für eine rasche Integration der aus humanitären Gründen eingewanderten Personen in den Arbeitsmarkt sollten daher verstärkt werden. 
... und die weiterhin bestehenden juristischen Hindernisse für den Zugang zum Arbeitsmarkt sollten beseitigt werden.

Der Rahmen für die Integration ist ziemlich komplex und undurchsichtig ...
Seit 2008 haben aus humanitären Gründen eingewanderte, vorläufig aufgenommene Migranten Zugang zum Arbeitsmarkt, ohne Prüfung der Arbeitsmarktsituation. Sie benötigen jedoch eine Arbeitsbewilligung, im Gegensatz zu den anderen Gruppen von Migranten. Diese erhalten sie im Allgemeinen, wenn sie einen Arbeitgeber finden, der bereit ist, sie einzustellen. Eine Abschaffung der Pflicht zum Erwerb einer separaten Arbeitsbewilligung würde dazu beitragen, dass der Papierkrieg vermindert und die Integration in den Arbeitsmarkt vereinfacht würde. Auf jeden Fall müssen die Arbeitgeber besser über die Möglichkeit der Anstellung solcher Migranten informiert werden.

Da die Tätigkeit des Bundes im Bereich der Integration bis vor kurzem eingeschränkt war, wurden die meisten Integrationsmassnahmen auf kantonaler oder kommunaler Ebene umgesetzt, was das Subsidiaritätsprinzip widerspiegelt. Dies hat zwar zu einer Flexibilität bei der Integration geführt, aber auch zu grossen Unterschieden bei den Dienstleistungen zwischen den einzelnen Kantonen. Auch wenn diese Tatsache in der Schweiz für viele Politiken gilt und auch zahlreiche Massnahmen und Programme für Nicht-Immigranten davon betroffen sind, stellt sich für Personen, die aus humanitären Gründen eingewandert sind, trotzdem die Frage nach der horizontalen Gerechtigkeit. Schliesslich können sie ihren Wohnkanton nicht frei wählen und sind auch in ihrer Mobilität eingeschränkt.

... was hauptsächlich darauf zurück Komplexität und Intransparenz des schweizerischen zu führen ist, dass der Zugang zu den Integrationsmassnahmen von vielen Faktoren abhängt.
Integrationsrahmens sind vielleicht dessen grösster Mangel. Dies ist im Wesentlichen darauf zurück zu führen, dass der Zugang zu den Integrationsdiensten von zahlreichen Faktoren abhängt - von Wohnkanton, Aufenthaltsstatus und Dauer des Aufenthalts, Erhalt (oder nicht) von Sozialversicherungsleistungen und davon, welche Sozialversicherung (Arbeitslosigkeit, Invalidität oder Sozialhilfe) gegebenenfalls für den Migranten zuständig ist. 
Die Unterschiede im Angebot der Dienstleistungen erfordern eine bessere Koordination des staatlichen Handelns.

Lohnsubventionen scheinen eine besonders wirksame Integrationsmassnahme für Immigranten zu sein, diese profitieren jedoch nur selten davon.
Die Tatsache, dass das Angebot an Integrationsdiensten in den verschiedenen Kantonen offenbar unterschiedlich ist und viele Migranten nicht von den allgemeinen Massnahmen profitieren können, erfordert eine bessere Koordination des staatlichen Handelns, sowohl innerhalb der Kantone als auch zwischen den Kantonen. Es sollten gemeinsame Minimalnormen angewendet werden, um sicher zu stellen, dass die Immigranten von Massnahmen profitieren können, die ihren Bedürfnissen entsprechen, und dies unabhängig vom Wohnort, dem Aufenthaltsstatus und der Art und Höhe von Versicherungsleistungen oder -beihilfen, auf die sie Anspruch haben oder nicht. Ausserdem wäre es wichtig, den Austausch guter Praktiken zwischen den Kantonen und Gemeinden zu verstärken.

Damit ein solcher Austausch Sinn macht, muss vorgängig evaluiert werden, welche Massnahmen funktionieren und welche nicht. Dies bedeutet, dass Forschung und Evaluation in die neuen Programme integriert werden müssen. Obwohl in der Schweiz die allgemeine Evaluationskultur gut entwickelt ist, gibt es praktisch keine spezifische Evaluation der Arbeitsmarktpolitik bezüglich der Immigranten und ihrer Kinder. Dies widerspiegelt den Ansatz einer Integrationspolitik über die allgemeinen Programme und Massnahmen, lässt jedoch die Tatsache beiseite, dass die Auswirkungen der aktiven Arbeitsmarktpolitik für Immigranten anders sein können als für Personen ohne Migrationshintergrund. Frühere Evaluationen (sowohl in der Schweiz als auch in anderen OECDLändern) haben ein ziemlich robustes Ergebnis gebracht: Lohnsubventionen wirken sich besonders bei Immigranten sehr positiv aus. In der Schweiz profitieren diese jedoch nur selten von dieser Massnahme.

Da die heute verfügbaren Daten von Eine spezifische Evaluation fehlt nicht nur bezüglich der besserer Qualität sind, sollte es Teil Programme für Immigranten, sondern auch allgemein in der einer kohärenten Integrationspolitik Forschung über Integration. Dies ist erstaunlich, wenn man sein, das Forschungsdefizit zu beheben. bedenkt, wie viele Immigranten und ihre Kinder seit langem in der Schweiz leben. Das Fehlen quantitativer Forschung ist teilweise auf einen Mangel an Daten zurück zu führen. Da nun nach und nach mehr und bessere Datensammlungen verfügbar werden (auch Längsschnittdaten), sollte das Beheben des Defizits in Forschung und Evaluation der Programme zur Integration der Immigranten und ihrer Kinder in den Arbeitsmarkt ein wichtiges Element beim Erarbeiten einer besser informierten und zielgerichteteren Politik Sein. 
Die Sprachförderung muss ausgebaut und stärker auf den Arbeitsmarkt ausgerichtet werden.
Internationale Studien zeigen, dass das Erlernen der Sprache die Integration der Immigranten erleichtern kann. Eine Evaluation der Sprachförderung in der Schweiz ist schwierig, da sie grösstenteils von verschiedenen Diensten auf kantonaler Ebene angeboten wird, die kaum miteinander in Kontakt stehen. Die wenigen vorhandenen Informationen über die sprachliche Förderung weisen darauf hin, dass entsprechende Kurse weniger häufig durchgeführt werden als in anderen OECD-Ländern, und der Besuch solcher Kurse nicht mit guten Resultaten in Bezug auf die Beschäftigung einhergeht. Es würde sich lohnen, die Gründe dafür vertieft zu untersuchen und die entsprechenden Massnahmen zu treffen. Auf jeden Fall scheint es vernünftig, das Feld der sprachlichen Ausbildung sowohl zu erweitern als auch stärker auf das Berufsleben auszurichten. Auch wäre es wünschenswert, die Schaffung eines allgemeinen Rahmens für die sprachliche Ausbildung ins Auge zu fassen und diese so gesamtschweizerisch $\mathrm{zu}$ vereinheitlichen.

Die Einbürgerung sollte erleichtert werden, da sie die Integration der Immigranten fördert.
Die Schweiz ist in Sachen Einbürgerungspolitik eines der restriktivsten Länder innerhalb der OECD. Diese Tatsache widerspiegelt die Idee, dass die Einbürgerung nicht ein Instrument für die Förderung der Integration ist, sondern vielmehr die Bestätigung einer gelungenen Integration. Gemäss den empirischen Daten geht die Einbürgerung Hand in Hand mit besseren Resultaten im Bereich der Beschäftigung der Immigranten und ihrer Kinder in der Schweiz. Dies gilt besonders für Immigranten aus Ländern mit niedrigen Einkommensniveau, die so Zugang erhalten zu besser qualifizierten Tätigkeiten und zum öffentlichen Dienst. Diese Feststellungen und weitere Längsschnittdaten aus OECD-Ländern legen nahe, dass mit einem erleichterten Zugang zum Schweizer Bürgerrecht offenbar viel zu gewinnen wäre. Auf jeden Fall hat das Beibehalten der geltenden Einschränkungen seinen Preis. Es wäre wichtig, dass diesbezüglich eine Sensibilisierung stattfinden würde und die Vorteile aufgezeigt würden, welche der Erwerb des Schweizer Bürgerrechts den dazu berechtigten Migranten bringt. 
Die Wohnsitzkriterien in Kanton und Gemeinde sollten überarbeitet werden, um die Mobilität zu fördern.
Die Integrationsmassnahmen müssen die Kompetenzen der Immigranten stärker berücksichtigen.
Neben der eidgenössischen Wohnsitzfrist von 12 Jahren für die ordentliche Einbürgerung gibt es Wohnsitzfristen des Kantons und der Gemeinde. Dies erschwert den Erwerb des Bürgerrechts und kann auf die Mobilität der Immigranten innerhalb der Schweiz bremsend wirken. Eine Reform der Schweizerischen Gesetzgebung über das Bürgerrecht ist in Vorbereitung. Sie sollte mittels einer umfassenden Harmonisierung der kantonalen und kommunalen Wohnsitzfristen einige der wichtigsten Schwächen des Gesetzes beseitigen und die eidgenössische Wohnsitzfrist auf acht Jahre verkürzen. Selbst wenn die vorgesehene Reform angenommen würde, wäre das schweizerische System im internationalen Vergleich weiterhin restriktiv, insbesondere für die zahlreichen in der Schweiz geborenen Kinder eingewanderter Eltern, von denen gegenwärtig nur wenige das Schweizer Bürgerrecht besitzen.

Die meisten verfügbaren Integrationsmassnahmen sind auf niedrig qualifizierte Arbeit ausgerichtet. Die Zahl der Migranten, die ihr Diplom in einem Land mit niedrigem Einkommensniveau erworben haben und «überqualifiziert» sind (Migranten in einer Anstellung, für die eine niedrigere Qualifikation verlangt wird als die formelle Qualifikation, die sie besitzen) ist jedoch hoch, und diese Feststellung scheint auch nach Berücksichtigung objektiver Kompetenzmessungen ihre Gültigkeit zu behalten.

Evaluation und Anerkennung ausländischer Diplome scheinen einer der Schwachpunkte des schweizerischen Systems zu sein. Die entsprechenden Kosten sind zwar tief und das Verfahren ist relativ schnell, das System ist jedoch nicht transparent und sogar bei Erbringern von Integrationsleistungen nicht sehr bekannt. Ausserdem ist es momentan für gewisse Berufe unmöglich, eine Evaluation und eine Anerkennung der Qualifikation und der im Ausland erworbenen Berufserfahrung zu erhalten. Diese Lücken im System sollten geschlossen werden. Da die überwiegende Mehrheit der Immigranten mit einem Diplom den Titel im Ausland erworben hat, ist es wichtig, das System für die Evaluation und die Anerkennung zu verbessern. Das Ergebnis des Evaluationsverfahrens scheint auf dem Arbeitsmarkt akzeptiert zu sein, weil die Immigranten, die eine formelle Anerkennung erhalten haben, im Bereich der Beschäftigung bessere Ergebnisse erzielen. Es gibt jedoch nur sehr wenige Immigranten mit einem Hochschuldiplom eines Nicht-EWR-Landes, die eine die Evaluation und Anerkennung ihrer Diplome beantragen. Es ist nicht ganz klar, ob dies darauf zurück zu führen ist, dass sie diese Möglichkeit nicht kennen, oder darauf, dass sie ein Gesuch gar nicht erst stellen, weil sie sich nur wenige Chancen auf eine Anerkennung ausrechnen. 
In dieser Hinsicht wäre es nützlich, Das Bundesamt für Berufsbildung und Technologie (BBT) eine zentrale Anmeldestelle für alle akzeptiert bereits «Vorgesuche» für Anerkennungen in allen Evaluations- und Disziplinen und auf allen Ebenen und bietet Informationen über Anerkennungsgesuche zu schaffen. die zuständigen Stellen an. Es wäre nun ein weiterer Schritt, eine zentrale Anmeldestelle zu schaffen, die formell alle Gesuche annimmt, statt sie den Kandidaten zurïck zu schicken und ihnen die zu kontaktierende Stelle anzugeben. Ein solches Vorgehen wäre ein wichtiger Fortschritt im Sinn der Transparenz. Ausserdem sollte die Tatsache, dass man mit einer Anerkennung leichter eine gute Stelle findet, unter den Immigranten stärker verbreitet werden.

Die Passerellen-Angebote und das Migranten sollten in grösserem Umfang umgesetzt werden, in Kooperation mit den Arbeitgebern. Mentoring für qualifizierte

Es gibt nur wenige Passerellen-Ausbildungen für Immigranten, und die Tatsache, dass der Schwerpunkt sowohl bei den Flüchtlingen als auch allgemein bei den Arbeitslosen auf eine schnelle Integration in den Arbeitsmarkt gelegt wird, steht im Widerspruch zu Brückenkursen bzw. Passerellen-Angeboten. Insbesondere der Zugang zu hochqualifizierten Stellen erfolgt oft über Netzwerke, aber über diese verfügt die eingewanderte Bevölkerung kaum. Hier scheinen Mentoring-Programme ein wirksames Instrument zu sein, diese sind jedoch in Umfang und Reichweite bislang begrenzt. Es scheint auch, dass die Immigranten sehr von einem grösseren Angebot für Akkreditierung nicht-formalen Lernens profitieren würden, einem Angebot, das in der Schweiz nach und nach ausgebaut wird. Bei der Ausarbeitung der Massnahmen zur Akkreditierung nichtformalen Lernens sollte sicher gestellt werden, dass diese die Immigranten tatsächlich erreichen und auf ihre Bedürfnisse zugeschnitten sind. Diese Massnahmen sollten verstärkt angeboten werden, in Ko-operation mit den Arbeitgebern.

Diskriminierung bei der Anstellung kommt in der Schweiz häufig vor, und eine verstärkte Sensibilisierung für dieses Problem ist erforderlich.
Durch Teststudien konnte gezeigt werden, dass Diskriminierung bei der Stellensuche häufig vorkommt. Um zu einem Vorstellungsgespräch eingeladen zu werden müssen immigrantenkinder, die ihre Ausbildung in der Schweiz absolviert haben, müssen bis zu fünf mal mehr Bewerbungen schreiben als Kinder ohne Migrationshintergrund mit ansonsten vergleichbarem Lebenslauf,. Die relativ tiefe Arbeitslosigkeit bei Immigrantenkindern lässt darauf schliessen, dass diese die Diskriminierung dadurch kompensieren, dass sie sich mehr bewerben. Die Häufigkeit der Diskriminierung wird auch teilweise dadurch überdeckt, dass die Lage auf dem Arbeitsmarkt allgemein gut ist. In der öffentlichen Debatte wird das Diskriminierung bei der Anstellung zudem kaum thematisiert. Dennoch benötigen Kinder von Immigranten doppelt so viel Zeit wie Kinder ohne Migrationshintergrund und ansonsten gleichen Schulleistungen, um eine Stelle zu finden. Eine stärkere Sensibilisierung für dieses Problem ist daher wichtig. 
Der juristische und institutionelle Rahmen für den Kampf gegen die Diskriminierung liegt im Vergleich mit den anderen OECD-Ländern weit zurück...

... und sollte durch andere Massnahmen ergänzt werden.
Der juristische und institutionelle Rahmen für den Kampf gegen die Diskriminierung liegt im Vergleich mit den meisten anderen OECD-Ländern weit zurück. Zurzeit ist Diskriminierung aufgrund der Nationalität nicht illegal, ausser für Immigranten aus der EU. Ausserdem sind die Beratungsstrukturen häufig weniger gut ausgestattet als in anderen Ländern. Sie können zwar juristische Stellungnahmen abgeben, jedoch keine Klagen einreichen. Dementsprechend ist die Anzahl der behandelten Dossiers unbedeutend angesichts der Häufigkeit von Diskriminierung, wie sie durch die Teststudien ans Licht gebracht worden ist.

Es ist daher wichtig, dass sowohl der juristische als auch der institutionelle Rahmen für den Kampf gegen Diskriminierung verstärkt und bei den Immigranten besser bekannt gemacht werden. Diese Reform könnte ergänzt werden durch entschlossenere Massnahmen zugunsten der Diversität, mit dem Ziel einer grösseren Vielfalt an Rekrutierungswegen für die Arbeitgeber. Dies fehlt momentan in der Schweiz. Der öffentliche Dienst, in dem die Immigranten untervertreten sind, muss bei dieser Bewegung ebenfalls involviert sein. Massnahmen wie beispielsweise anonyme Lebensläufe, «Diversitätslabels» und Beratung zu Diversität wurden vor kurzem in mehreren OECD-Ländern umgesetzt.
Das System der Berufslehre scheint für die Kinder der Immigranten gut zu funktionieren aber der Tatsache, dass der Prozentsatz der Lehrabschlüsse niedrig ist, sollte mehr Beachtung geschenkt werden.
Das internationale Programm der OECD zur Schülerbewertung (PISA) hat gezeigt, dass Immigrantenkinder in der Schweiz im Alter von 15 Jahren bedeutend schlechtere schulische Leistungen erbringen als die Kinder ohne Migrationshintergrund, und dass der Abstand grösser ist als in vielen anderen OECD-Ländern. Eine Längsschnittanalyse mit Schweizer PISA-Daten weist darauf hin, dass das Schul- und Ausbildungssystem diesen Immigrantenkindern später trotzdem relativ gute Qualifikationen ermöglicht. Das System der Berufslehre scheint für sie besonders gut zu funktionieren. Die Wahrscheinlichkeit, dass Immigrantenkinder ihre Lehre auch abschliessen, ist allerdings weniger gross. Die Gründe für dieses Phänomen sollten näher untersucht und anschliessend die nötigen Korrekturmassnahmen getroffen werden. 
Immigrantenkinder scheinen heute mehr Probleme zu haben als frïher. Diesem Missstand abzuhelfen, sollte eine Priorität des staatlichen Handelns werden.

Alle jungen Immigranten müssen gleichberechtigten Zugang zur aktiven Arbeitsmarktpolitik für Jugendlichehaben.
Die meisten Immigrantenkinder schaffen den Übergang von der Schule ins Erwerbsleben gut. Es gibt jedoch Anzeichen, die darauf hindeuten, dass die neuen Kohorten von Immigrantenkindern heute grössere Schwierigkeiten haben als frühere Gruppen, und viele im Ausland geborene Immigrantenkinder erzielen weniger gute Leistungen. Dies betrifft insbesondere Kinder, deren Eltern aus Ländern mit niedrigen Einkommensniveau stammen. Einige Jahre nach Schulabschluss haben sie oft weder eine Stelle noch stehen sie in einer Ausbildung (NEET). Da heute viele Kinder von Immigranten aus Ländern mit niedrigen Einkommensniveau ins Berufsleben eintreten, sollte die öffentliche Hand die Verbesserung ihrer Integrationsergebnisse $\mathrm{zu}$ einer Priorität machen.

Es existiert eine breite Palette an Massnahmen, um Jugendlichen, die Unterstützung brauchen, den Übergang von der Schule ins Erwerbsleben zu erleichtern. Da der Zugang zu den meisten Massnahmen der aktiven Arbeitsmarktpolitik jedoch häufig auf Personen beschränkt ist, die sich seit mehr als 10 Jahren im Land befinden, sind viele junge Immigranten davon ausgeschlossen. Ausserdem erhalten die jungen Immigranten, die von diesen Programmen, insbesondere dem «Motivationssemester» profitieren, keine Arbeitslosenbeiträge (im Gegensatz zu den in der Schweiz geborenen Kindern). Sie können daher versucht sein, direkt in den Arbeitsmarkt einzutreten und sich mit einer wenig qualifizierten Arbeit zufrieden zu geben. So stehen sie in der Gefahr, später ohne Stelle und ohne Ausbildung dazustehen , was offenbar häufig der Fall ist. Es wäre eine erste Massnahme, um diese wichtige Herausforderung anzugehen, wenn sicher gestellt würde, dass Immigrantenkinder dieselben Anreize für die Teilnahme an den Programmen haben wie Kinder ohne Migrationshintergrund.

Die Förderung und Betreuung der Die vorschulische Erziehung ist offenbar ein Bereich, der für Kleinkinder muss verbessert werden, Immigrantenkinder besondere Vorteile mit sich bringt. Dies zeigen mit einem Schwerpunkt auf Kinder Forschungen aus mehreren OECD-Ländern. Die vorschulische benachteiligter Immigranten.

Erziehung ist jedoch in der Schweiz gegenwärtig stark unterentwickelt, insbesondere für Kinder im für die Integration entscheidenden Alter von drei und vier Jahren. Ein grösseres und zielgerichteteres Angebot vorschulischer Erziehung für diese Altersgruppe sowie Massnahmen für eine frühe sprachliche Unterstützung würden offenbar viele Vorteile bringen. Profitieren würden nicht nur die Kinder der Immigranten, sondern auch deren Mütter, für welche die Tatsache, Kinder zu haben, einen viel grösseren Einfluss auf den Bereich der Erwerbstätigkeit hat als für Mütter, die in der Schweiz geboren sind. 
DELSA/ELSA/WD/SEM(2012)2

\section{ANNEXE 3.}

\section{SINTESI}

La Svizzera è uno dei Paesi dell'OCSE con la maggiore percentuale di immigrati (il 27\% delle persone in età lavorativa è nato all'estero) e in cui il tema dell'immigrazione occupa un posto di rilievo sia nell'agenda politica sia nel dibattito pubblico. Se si tiene conto delle numerose discussioni sollevate da questo problema a livello nazionale, si potrebbe essere tentati di pensare che in Svizzera le persone immigrate siano meno bene integrate che in altri Paesi.

I fatti dimostrano invece che, nell'insieme, l'integrazione in Svizzera funziona piuttosto bene. In genere, per quanto riguarda il mercato del lavoro, la popolazione immigrata si trova in una situazione complessivamente molto favorevole rispetto a quanto si osserva in altri Paesi. Il tasso di occupazione degli immigrati di ambedue i sessi è più elevato che negli altri Paesi dell'OCSE.

Questi ottimi risultati sono da ricondurre soprattutto alla situazione del mercato del lavoro svizzero globalmente buona e a una combinazione particolare di Paesi d'origine. La maggior parte dei migranti (più del 60\%) è originaria di Paesi dell'OCSE ad alto reddito, e più della metà di loro proviene dai Paesi limitrofi, le cui lingue nazionali sono le stesse di quelle parlate in Svizzera. Gli altri immigrati provengono soprattutto dai Paesi dell'ex Jugoslavia e dalla Turchia.

Negli ultimi anni, dopo l'introduzione progressiva della libera circolazione delle persone con gli Stati membri dell'Unione europea, la Svizzera ha assistito all'arrivo di un numero insolitamente elevato di stranieri. Circa il 5\% della popolazione residente è composto da immigrati recenti ossia da persone che si sono stabilite in Svizzera da meno di cinque anni. La maggior parte dei nuovi arrivati è originaria dei Paesi limitrofi, soprattutto dalla Germania e, secondo tutti gli indicatori convenzionali, si integra bene nel mercato del lavoro.

Tuttavia, nonostante un quadro globalmente positivo, la situazione è più delicata per certe categorie di immigrati, come per esempio le donne con bambini piccoli. Infatti, sono poche le iniziative a favore dell'integrazione delle donne immigrate, le quali non hanno accesso a tutte le misure di politica attiva del mercato del lavoro. Del resto, alcuni elementi denotano un calo del tasso d'attività di questo gruppo di popolazione da qualche anno a questa parte.

Vi è un altro gruppo con un tasso d'occupazione mediocre, anche nel confronto internazionale: i migranti titolari di un permesso di soggiorno per motivi umanitari recentemente arrivati che, rispetto ai gruppi precitati, sembrano avere maggiori difficoltà a integrarsi nel mercato del lavoro svizzero. Contrariamente ad altri Paesi, la Svizzera non ha ancora predisposto un programma d'integrazione specifico destinato a questa categoria di nuovi arrivati, il che può spiegare in parte tali risultati insoddisfacenti. Sulla scorta delle esperienze incoraggianti maturate dai Paesi dell'OCSE che propongono programmi d'integrazione strutturati e incentrati sul mercato del lavoro, sarebbe auspicabile che la Svizzera prendesse seriamente in considerazione l'adozione di simili iniziative.

In Svizzera, il tasso d'occupazione generalmente elevato degli immigrati è legato anche al fatto che $\mathrm{i}$ migranti titolari di un diploma provenienti da Paesi non membri dell'OCSE spesso sono sovraqualificati. Le qualifiche acquisite all'estero sono insufficientemente valorizzate sul mercato del lavoro svizzero, e l'offerta di corsi "passerella" è alquanto limitata. Contrariamente ad altri Paesi dell'OCSE, la Svizzera ha realizzato pochi programmi di mentorato o iniziative analoghe atti a fornire agli immigrati i contatti indispensabili con gli autoctoni e i datori di lavoro, familiarizzandoli al tempo stesso con il funzionamento del mercato del lavoro. Vista la cruciale importanza di questi due aspetti per accedere a impieghi altamente 
qualificati, simili interventi meriterebbero di essere ampiamente diffusi in collaborazione con i datori di lavoro.

Il carattere federale del Paese si riflette chiaramente nella politica d'integrazione. Infatti, diverse misure volte a promuovere l'integrazione sono state sviluppate a livello locale e cantonale. L'applicazione del principio della sussidiarietà ha comportato l'attuazione di misure puntuali e flessibili destinate a numerosi immigrati. Ma al tempo stesso ha ritardato lo sviluppo di una politica integrativa a livello federale. Di conseguenza, e nonostante un netto miglioramento durante l'ultimo decennio, l'architettura generale in materia d'integrazione rimane sottosviluppata. Paragonandola alle azioni condotte in altri Paesi dell'OCSE, nella maggior parte dei quali la popolazione immigrata è meno numerosa che in Svizzera, la politica integrativa federale è abbastanza modesta. Salvo qualche strumento come la formazione linguistica elementare finanziata dall'Ufficio federale della migrazione, le misure specifiche destinate agli immigrati in Svizzera sono poche. Invece di concepire politiche destinate ai soli immigrati, l'approccio globale all'integrazione parte dall'idea che gli immigrati possono beneficiare dei servizi offerti a tutti. Per colmare le lacune del sistema attuale, la Confederazione, i Cantoni e le autorità locali hanno formulato recentemente un certo numero di suggerimenti volti a migliorare il quadro generale dell'integrazione, impegnandosi inoltre ad aumentare le risorse destinate all'integrazione.

A livello cantonale, le misure volte a favorire l'integrazione variano molto, in parte a causa delle differenze per quanto riguarda entità e composizione delle popolazioni immigrate. Sebbene negli ultimi anni numerosi Cantoni abbiano intensificato le loro misure integrative, spesso lo hanno fatto su piccola scala e nell'ambito di progetti modello, il che non permette di valutarne facilmente l'efficacia. Occorrerebbe che le autorità federali stabilissero alcune norme minime affinché tutti gli immigrati possano beneficiare delle misure di cui hanno bisogno, a prescindere dal loro Cantone di residenza.

Per gli immigrati è difficile accedere alla cittadinanza svizzera: in effetti, la durata di soggiorno richiesta è particolarmente lunga - 12 anni per la procedura ordinaria, ossia il periodo più lungo dell'OCSE -, inoltre il processo d'acquisizione si svolge a tre livelli e il candidato deve soddisfare le esigenze federali, cantonali e comunali. $\grave{E}$ in atto una riforma della legislazione, volta a ovviare alle maggiori lacune della legge sulla cittadinanza e a migliorare la mobilità dei migranti sul territorio svizzero. Le osservazioni empiriche suggeriscono che ciò potrebbe dare una forte spinta all'integrazione dei gruppi di immigrati svantaggiati.

Nel confronto internazionale, le performance occupazionali dei figli d'immigrati sono generalmente buone. Ciò è dovuto in parte alle buone condizioni generali del mercato del lavoro nonché ad altri fattori quali il ruolo importante dell'apprendistato che, a quanto pare, avrebbe un effetto particolarmente benefico per i figli di immigrati durante il processo di transizione dalla scuola al lavoro. Per preparare all'apprendistato i giovani poco scolarizzati (tra cui anche molti figli d'immigrati) sono altresì stati sviluppati alcuni programmi innovativi, che sembra abbiano avuto un effetto benefico.

I figli d'immigrati i cui genitori hanno un basso livello d'istruzione conseguono risultati mediocri a scuola; al momento, questi giovani sono sempre più numerosi ad accedere al mercato del lavoro. Queste performance meno soddisfacenti sembrerebbero riconducibili, almeno in parte, alla mancanza di interventi di sostegno sufficientemente precoci da parte del sistema educativo. Offrire ai figli d'immigrati dall'età di tre o quattro anni in poi un insegnamento adeguato e mirato, parallelamente all'apprendimento della lingua, dovrebbe dunque imporsi urgentemente come una priorità dell'azione pubblica.

Un settore in cui la Svizzera accusa un certo ritardo rispetto ad altri Paesi dell'OCSE è quello della lotta alla discriminazione. I datori di lavoro non si rendono sufficientemente conto del problema, che del resto è assente anche dal dibattito pubblico. Eppure, alcuni studi hanno dimostrato che prima di essere invitato a un colloquio di assunzione, un figlio d'immigrati, soprattutto se i suoi genitori sono originari dell'ex Jugoslavia, deve presentare un numero di candidature pari al quintuplo di quelle inoltrate da un 
figlio di autoctoni. Occorrerebbe dunque adottare le misure che s'impongono per ovviare a questo tipo di ineguaglianza.

Sebbene, per quanto riguarda l'integrazione nel mercato del lavoro della popolazione immigrata, i risultati conseguiti dalla Svizzera nel confronto internazionale siano in genere buoni, alcuni segni rivelano una situazione diversa per determinate categorie di migranti, ossia per certi gruppi svantaggiati che corrono il rischio di restare indietro. È importante agire subito, mentre i risultati globali sono ancora positivi. Il problema è stato individuato e sono già in atto misure volte a intensificare l'integrazione ai tre livelli del governo. Per molti versi, le politiche integrative della Svizzera non reggono tuttavia il confronto con quelle adottate da altri Paesi dell'OCSE. Occorrerebbe pertanto considerare un certo numero di misure per rimediare a questa situazione e fare in modo che d'ora in poi i risultati siano soddisfacenti per tutte le categorie d'immigrati.

\section{Sintesi delle principali raccomandazioni destinate per le politiche}

\section{A) Potenziare il quadro globale d'integrazione}

- Sviluppare norme minime comuni affinché le misure volte a favorire l'integrazione sia applicate in tutti i Cantoni.

- $\quad$ Facilitare lo scambio di buone pratiche tra i Cantoni e i Comuni.

- Assicurarsi che tutti gli immigrati che necessitano di un aiuto all'integrazione vi possano accedere in modo appropriato, a prescindere dal tipo di permesso di cui sono titolari nonché dalla natura e dalla rilevanza delle prestazioni di cui beneficiano, specialmente trattandosi di donne immigrate.

- Offrire una formazione linguistica a tutti gli immigrati che ne hanno bisogno, tenendo conto delle loro competenze e delle loro qualifiche.

- $\quad$ Facilitare l'acquisizione della cittadinanza svizzera, in particolare riducendo le esigenze dei Cantoni e dei Comuni in termini di durata del soggiorno, così da agevolare la mobilità geografica dei migranti.

- Sensibilizzare ai vantaggi derivanti dall'acquisizione della cittadinanza svizzera per una migliore integrazione degli immigrati e dei loro figli.

\section{B) Favorire l'integrazione rapida nel mercato del lavoro dei migranti per motivi umanitari}

- $\quad$ Rafforzare gli incentivi dei Cantoni a favore dell'integrazione rapida nel mercato del lavoro dei migranti per motivi umanitari durante i primi cinque anni di soggiorno.

- $\quad$ Realizzare un programma d'integrazione strutturato destinato a tutte le persone immigrate di recente per motivi umanitari (i richiedenti la cui domanda è stata accolta o che beneficiano dell'ammissione provvisoria), tenendo conto dei bisogni individuali e ponendo chiaramente l'accento sull'integrazione nel mercato del lavoro.

- Informare meglio i datori di lavoro circa l'accesso al mercato del lavoro delle persone che beneficiano dell'ammissione provvisoria. 


\section{Sintesi delle principali raccomandazioni per le politiche (cont.)}

\section{C) Utilizzare al meglio le competenze dei migranti}

- Assicurarsi che l'accento attualmente posto sull'occupazione poco qualificata dei migranti per motivi umanitari non impedisca di utilizzare al meglio le loro competenze.

- Far meglio conoscere agli immigrati le offerte disponibili in materia di valutazione e di riconoscimento delle qualifiche acquisite all'estero e spiegare quali sono i vantaggi derivanti da tale riconoscimento.

- $\quad$ Predisporre corsi passerella e altri programmi di sostegno per aiutare gli immigrati titolari di diplomi conseguiti all'estero ad accedere a impieghi più qualificati.

- Considerare la possibilità di estendere con discernimento il beneficio delle sovvenzioni salariali temporanee agli immigrati.

\section{D) Intensificare gli sforzi a favore di una rapida integrazione dei figli d'immigrati}

- Sviluppare l'educazione prescolare e rivolgere un'attenzione particolare alla partecipazione dei figli d'immigrati provenienti da ambienti svantaggiati a partire dall'età di 3 o 4 anni.

- Potenziare la formazione linguistica dei figli d'immigrati, segnatamente dei più giovani.

- Esaminare le cause dei tassi apparentemente deboli di completamento della formazione da parte dei figli d'immigrati, adottare misure per porre rimedio a questa situazione.

\section{E) Stabilire un quadro solido per combattere la discriminazione}

- Rendere illegale la discriminazione fondata sulla nazionalità al momento dell'assunzione.

- Sensibilizzare soprattutto i datori di lavoro e la società in generale alla questione della discriminazione.

- $\quad$ Prendere in considerazione l'introduzione di misure più incisive per combattere la discriminazione. 


\section{VALUTAZIONE E RACCOMANDAZIONI}

La Svizzera presenta un'elevata percentuale di immigrati e, per quanto riguarda l'integrazione nel mercato del lavoro, il bilancio globale è positivo.
In Svizzera, le persone nate all'estero rappresentano circa il $27 \%$ della popolazione, il che corrisponde, come nel Lussemburgo e in Australia, a una delle percentuali più elevate registrate nella zona dell'OCSE. Nel confronto internazionale, i principali indicatori dell'integrazione degli immigrati nel mercato del lavoro sono in genere abbastanza favorevoli. La Svizzera si colloca tra i Paesi dell'OCSE con il più alto tasso d'occupazione degli immigrati di ambedue i sessi. Lo stesso vale anche per i figli nati in Svizzera da genitori immigrati. Tuttavia, nonostante il basso tasso di disoccupazione generale registrato nel Paese, la disoccupazione di certi gruppi di immigrati non è trascurabile, in particolare trattandosi in molti casi di donne immigrate con bambini piccoli.

Ciò è dovuto in parte alla situazione Pare che la situazione piuttosto favorevole dell'integrazione in di quasi pieno impiego in Svizzera e Svizzera rispetto agli altri Paesi dell'OCSE sia sostanzialmente inoltre al fatto che la maggior parte dovuta a due fattori. Innanzitutto, la situazione globale del mercato degli immigrati proviene dai Paesi del lavoro svizzero è buona. Inoltre, contrariamente alla maggior limitrofi. parte degli altri Paesi dell'OCSE, una forte proporzione dell'immigrazione passata e presente proviene da Paesi ad alto reddito, soprattutto dalla Germania e dall'Italia (i due maggiori Paesi d'origine). Nel gruppo dei Paesi d'origine a basso reddito, quasi la metà di tutti gli immigrati è originaria dell'ex Jugoslavia e dei suoi Paesi successori, senza dimenticare il 12\% degli immigrati provenienti dalla Turchia. I flussi migratori sono dovuti soprattutto a motivi di lavoro e al ricongiungimento familiare connesso, mentre l'immigrazione per motivi umanitari (che in tutti i Paesi è caratterizzata da maggiori difficoltà di integrazione nel mercato del lavoro) è più bassa rispetto ad altri Paesi. Tuttavia, in termini quantitativi, nemmeno questo gruppo è trascurabile e di fatto, negli ultimi due decenni, la Svizzera è stata uno dei principali Paesi di destinazione dei richiedenti l'asilo dell'OCSE. 
La Svizzera annovera numerosi

Dal 2002, in seguito alla progressiva introduzione della libera immigrati recenti originari dell'UE. circolazione delle persone con l'UE e i suoi Paesi membri, la In genere, si tratta di una fetta della Svizzera ha assistito all'afflusso di un forte numero di immigrati popolazione bene integrata nel mercato del lavoro. provenienti dall'UE. Gli immigrati arrivati durante questo periodo rappresentano una percentuale della popolazione del Paese superiore al 5\%. All'interno di questo gruppo si riscontra, secondo il Paese d'origine, una grande diversità sociodemografica dei flussi e segnatamente gli immigrati provenienti dal Portogallo sono confrontati anche con un elevato tasso di disoccupazione. In genere, le persone immigrate di recente in provenienza dall'UE allargata conseguono ottimi risultati sul mercato del lavoro. Rispetto alla media degli autoctoni, queste persone occupano più frequentemente un posto di lavoro equivalente al loro livello di qualifica formale, il che dimostra che le loro competenze corrispondono al fabbisogno di manodopera.

Vi sono segni di un degrado della Le donne immigrate originarie di Paesi a basso reddito, situazione occupazionale delle segnatamente le nuove arrivate, non solo conseguono risultati donne immigrate originarie di Paesi mediocri sul piano occupazionale, ma da alcuni anni vedono la loro a basso reddito. Occorre seguire la situazione addirittura deteriorarsi. Tale evoluzione è inquietante e cosa con particolare attenzione. richiede un attento monitoraggio. Infatti, le donne immigrate tendono in genere a sottrarsi agli sforzi compiuti in Svizzera a favore dell'integrazione, poiché spesso sono lontane dal mercato del lavoro e non beneficiano di alcuna prestazione. La recente riduzione del cofinanziamento (attraverso l'assicurazione contro la disoccupazione) delle misure attive del mercato del lavoro adottate a sostegno delle persone che non beneficiano di un'indennità di disoccupazione potrebbe comportare, a meno che i servizi sociali non si oppongano, una nuova riduzione dell'offerta già limitata di misure integrative, svantaggiando ulteriormente questo gruppo. Contrariamente ai migranti per motivi umanitari, i migranti giunti in Svizzera ai fini del ricongiungimento familiare non possono beneficiare di un aiuto all'integrazione standard all'arrivo, anche se viene offerto loro un certo sostegno sotto forma di una formazione linguistica di base. Gli altri Paesi dell'OCSE prevedono invece sempre più spesso misure d'accoglienza destinate ai nuovi arrivati, e/o le attività di organizzazioni non governative compensano l'assenza di offerte globali in tali Stati. Tali misure e attività occupano un posto meno importante in Svizzera. Siccome l'integrazione riuscita delle donne immigrate nel mercato del lavoro ha ricadute positive sui figli, l'ampliamento delle misure integrative destinate a questa cerchia della popolazione meriterebbe di essere preso seriamente e urgentemente in considerazione. 
La situazione mediocre delle persone immigrate di recente in Svizzera per motivi umanitari richiede misure più strutturate a favore dell'integrazione.
Le persone immigrate di recente in Svizzera per motivi umanitari conseguono risultati di gran lunga inferiori, sul mercato del lavoro, rispetto al passato e al confronto internazionale. Le ragioni non sono del tutto chiare. A ogni modo, si tratta di un gruppo che necessita di misure integrative mirate. Anche altri Paesi dell'OCSE hanno predisposto programmi strutturati volti a favorire l'integrazione di questo gruppo. Le misure adottate, che possono estendersi sull'arco di tre anni, sembrano dare buoni risultati. Il miglioramento della situazione dei migranti arrivati di recente in Paesi dotati di programmi strutturati incentrati sull'integrazione nel mercato del lavoro, quali la Danimarca e la Norvegia, induce a pensare che la Svizzera avrebbe molto da guadagnare lasciandosi ispirare da tale approccio.

Occorre potenziare gli incentivi dei Cantoni a una rapida integrazione dei migranti per motivi umanitari nel mercato del lavoro.

Sebbene competa alle amministrazioni sub-federali, l'aiuto sociale a livello cantonale è finanziato dalla Confederazione durante i primi cinque anni di soggiorno dei rifugiati. Per questo (viepiù) cospicuo gruppo di persone ammesse a titolo provvisorio, la Confederazione offre addirittura un finanziamento per i primi sette anni, prima ancora che i Cantoni / i Comuni prendano in carico le persone appartenenti a questo gruppo. Inoltre, le persone ammesse a titolo provvisorio beneficiano in minor misura dell'aiuto sociale normalmente previsto e pare che questo fenomeno vada di pari passo con una diminuzione degli sforzi volti a favorire l'integrazione di questo gruppo. Ne consegue uno scarso incentivo per i Cantoni a integrare nel mercato del lavoro i migranti giunti in Svizzera per motivi umanitari durante i primi cinque-sette anni. Ma se durante questo lasso di tempo non vengono preparati correttamente, per gli immigrati risulta estremamente difficile posizionarsi con successo sul mercato del lavoro. Secondo i dati di altri Paesi dell'OCSE, un'entrata rapida nel mercato del lavoro è determinante per l'integrazione a lungo termine. Occorre dunque potenziare gli incentivi per una rapida integrazione nel mercato del lavoro di questi migranti.

Dal 2008, i migranti per motivi umanitari ammessi a titolo provvisorio possono accedere al mercato del lavoro a prescindere dalla situazione occupazionale. Tuttavia, contrariamente agli altri gruppi, questi migranti necessitano tuttora di un'autorizzazione di lavoro che in genere riescono a ottenere se trovano un datore di lavoro disposto ad assumerli. Abolire l'obbligo di un'autorizzazione di lavoro a parte permetterebbe di ridurre le pratiche burocratiche e di facilitare l'integrazione nel mercato del lavoro. In ogni caso, occorre informare meglio i datori di lavoro della possibilità di assumere questi migranti. 


\section{Il contesto dell'integrazione $\grave{e}$ relativamente complesso e poco trasparente...}

Fino a poco tempo fa, gli interventi della Confederazione a favore dell'integrazione erano limitati, per cui la maggior parte delle misure integrative era realizzata a livello cantonale o comunale, secondo il principio della sussidiarietà. Pur avendo contribuito a un approccio flessibile all'integrazione, ciò ha condotto anche a una differenza rilevante tra i livelli di prestazione dei servizi nei vari Cantoni. Sebbene in Svizzera tale sia il caso in molti ambiti politici e in numerosi servizi destinati ai non immigrati, nel caso dei migranti per motivi umanitari ciò solleva la questione dell'equità orizzontale, visto che non possono scegliere liberamente il loro Cantone di residenza e possono beneficiare soltanto di una mobilità territoriale limitata.

...il che è dovuto soprattutto al fatto Il carattere assai complesso e poco trasparente del contesto che l'accesso ai servizi volti a dell'integrazione svizzero è forse il suo maggior difetto. Alla base vi favorire l'integrazione dipende da numerosi fattori. è Sostanzialmente il fatto che l'accesso ai servizi volti a favorire l'integrazione dipende da numerosi fattori - dal Cantone di residenza, dal tipo di permesso e dalla durata del soggiorno, dall'ottenimento (o meno) di prestazioni d'assicurazione sociale nonché dal regime d'assicurazione sociale (disoccupazione, invalidità o aiuto sociale) cui soggiace il migrante. Queste differenze tra Cantoni di residenza riguardano parimenti la popolazione autoctona, ma diversamente da quest'ultima, visto che si tratta soprattutto di migranti titolari di un permesso di soggiorno per motivi umanitari, gli immigrati non hanno la possibilità di scegliere liberamente il loro luogo di residenza a causa delle restrizioni alla mobilità geografica.

La differenza per quanto riguarda l'offerta di servizi evidenzia la necessità di coordinare maggiormente l'operato pubblico.
L'apparente differenza per quanto riguarda l'offerta di servizi volti a favorire l'integrazione nei vari Cantoni e il fatto che numerosi migranti non possono beneficiare dei servizi generalmente offerti evidenziano la necessità di un maggiore coordinamento dell'operato pubblico, sia all'interno dei Cantoni sia tra i vari Cantoni. Occorrerebbero norme minime comuni per garantire agli immigrati misure corrispondenti alle loro esigenze, a prescindere dal loro luogo di residenza in Svizzera, dal tipo di permesso nonché dalla natura e dalla rilevanza delle prestazioni/delle indennità assicurative a cui hanno o non hanno diritto. Andrebbe inoltre rafforzato lo scambio di buone pratiche tra i Cantoni e i Comuni. 
Apparentemente le sovvenzioni salariali sono una misura integrativa particolarmente efficace destinata agli immigrati, di cui questi tuttavia beneficiano raramente.

Affinché il predetto scambio abbia una sua utilità, occorre valutare preliminarmente le misure che funzionano e quelle che non funzionano, il che implica la necessità di integrare nei nuovi programmi le dimensioni della ricerca e della valutazione. Infatti, nonostante una cultura di valutazione globale ben sviluppata in Svizzera, non vi è stata praticamente nessuna valutazione specifica delle politiche del mercato del lavoro concernente gli immigrati e i loro figli. Ciò riflette l'approccio alla politica d'integrazione attraverso i servizi generalmente offerti, ma non tiene conto del fatto che la politica attiva del mercato del lavoro può avere un impatto differente sugli immigrati e sugli autoctoni. Dalle valutazioni effettuate in passato (sia in Svizzera sia in altri Paesi dell'OCSE) è scaturito un risultato abbastanza concreto: le sovvenzioni salariali sono particolarmente benefiche per gli immigrati. Peccato che in Svizzera questi ultimi beneficino solo raramente di tale misura.

\begin{abstract}
Dal momento che i dati ora disponibili sono di migliore qualità, una politica d'integrazione coerente dovrebbe dunque colmare la lacuna della ricerca.
\end{abstract}

L'assenza di una valutazione specifica dei programmi concernenti gli immigrati vale anche, sebbene in un'ottica più generale, per la ricerca sull'integrazione, il che sorprende vista la forte presenza di lunga data degli immigrati e dei loro figli in Svizzera. L'assenza di una ricerca quantitativa è dovuta in parte a una mancanza di dati. Dal momento che le serie di dati rese progressivamente disponibili sono sempre più numerose e di migliore qualità (compresi i dati longitudinali), cercare di colmare la lacuna della ricerca e rimediare alla mancante valutazione dei programmi concernenti l'integrazione nel mercato del lavoro degli immigrati e dei loro figli dovrebbe costituire un elemento importante per l'elaborazione di strategie politiche più chiare e più mirate.

A livello internazionale è risaputo che l'apprendimento della lingua può facilitare l'integrazione degli immigrati. È difficile valutare la formazione linguistica in Svizzera dal momento che è perlopiù dispensata a livello cantonale, da servizi che praticamente non interagiscono tra loro. Le poche informazioni disponibili sulla formazione linguistica inducono a pensare che in Svizzera l'offerta sia più limitata che negli altri Paesi dell'OCSE e non venga associata a buoni risultati per quanto riguarda l'occupazione di coloro che l'hanno conseguita. Occorrerebbe esaminare in modo più approfondito le ragioni a monte e adottare misure corrispondenti. Ad ogni modo sarebbe d'uopo ampliare il campo della formazione linguistica e improntare maggiormente l'insegnamento alla professione. Per standardizzare la formazione linguistica su tutto il territorio svizzero sarebbe auspicabile un quadro globale. 


\section{Occorrerebbe facilitare la naturalizzazione in quanto fattore d'integrazione dei migranti.}

Tra i Paesi dell'OCSE, la Svizzera si è dotata di una delle politiche di naturalizzazione più restrittive, rispecchiando in questo l'idea della naturalizzazione intesa come garanzia di un'integrazione riuscita e non come uno strumento atto a promuoverla. Secondo i dati empirici, la naturalizzazione viene associata a migliori risultati per quanto riguarda la situazione occupazionale degli immigrati e dei loro figli in Svizzera, in particolare trattandosi di persone originarie di Paesi a basso reddito che in tal modo possono accedere a impieghi più qualificati e svolgere funzioni pubbliche. Partendo da tali constatazioni e da altri dati longitudinali forniti dai Paesi dell'OCSE, si può ipotizzare che ci sarebbe molto da guadagnare facilitando l'acquisizione della cittadinanza svizzera. Il mantenimento delle restrizioni in vigore ha comunque un costo e pertanto sarebbe opportuno intraprendere un'azione di sensibilizzazione in merito, dimostrando i vantaggi connessi all'ottenimento della cittadinanza svizzera per i migranti che vi hanno diritto.

Oltre al requisito federale di 12 anni di soggiorno per la naturalizzazione ordinaria, esistono requisiti di residenza a livello cantonale e comunale. Ciò rende più difficile l'acquisizione della cittadinanza e può essere un freno alla mobilità degli immigrati all'interno del Paese. $\grave{E}$ in preparazione una riforma della legislazione svizzera sulla naturalizzazione. L'obiettivo è di eliminare alcuni dei maggiori punti deboli della legge grazie a un'armonizzazione globale dei requisiti relativi alla durata di residenza stabiliti a livello cantonale e comunale e di ridurre a otto anni la durata di soggiorno richiesta a livello federale. Anche se la riforma prevista sarà votata, nel confronto internazionale il sistema svizzero resterà comunque restrittivo, soprattutto per quanto riguarda i numerosi bambini nati in Svizzera da genitori immigrati di cui attualmente solo alcuni hanno la cittadinanza svizzera.

La maggior parte delle misure volte a favorire l'integrazione sono incentrate sul lavoro poco qualificato. Molti migranti che hanno conseguito un diploma in un Paese a basso reddito sono sovraqualificati e svolgono mansioni corrispondenti a una qualifica inferiore a quella formalmente conseguita. Questa constatazione sembra mantenersi anche se si tiene conto di una valutazione obiettiva delle competenze. 
Occorre accrescere la trasparenza della valutazione e del riconoscimento dei diplomi esteri e considerare tutte le professioni.
Sarebbe utile creare uno sportello unico per la presentazione di tutte le richieste di valutazione e di riconoscimento.
La valutazione e il riconoscimento dei diplomi esteri sembrano uno dei punti deboli del sistema svizzero. Sebbene i relativi costi siano bassi e il processo abbastanza veloce, il sistema manca di trasparenza ed e inoltre poco conosciuto anche dai fornitori di prestazioi integrative. Del resto, per un certo numero di professioni è tuttora impossibile ottenere una valutazione e un riconoscimento delle qualifiche e dell'esperienza lavorativa acquisite all'estero. Proprio qui risiedono le lacune del sistema che andrebbero colmate. È importante migliorare il sistema di valutazione e di riconoscimento, poiché la stragrande maggioranza degli immigrati diplomati ha conseguito il proprio titolo all'estero. A quanto pare, il risultato di tale procedura è bene accetto sul mercato del lavoro, giacché gli immigrati che hanno ottenuto un riconoscimento formale dei loro titoli mostrano risultati migliori sul piano occupazionale. Comunque, sono rari gli immigrati in possesso di diplomi d'insegnamento superiore rilasciati da Paesi non membri dello SEE che chiedono la valutazione e il riconoscimento dei loro titoli. Non si capisce bene se ciò sia dovuto alla loro ignoranza di tale possibilità o se sono semplicemente scoraggiati all'idea di dover presentare la richiesta, ritenendo di avere poche probabilità di ottenere il riconoscimento.

L'Ufficio federale della formazione professionale e della tecnologia (UFFT) accetta già le «domande preliminari» di riconoscimento in tutte le discipline e a tutti i livelli e fornisce informazioni sugli organi competenti. In una prossima tappa, si potrebbe creare uno sportello unico che accetti formalmente tutte le richieste anziché rinviarle ai candidati indicando il nome dell'organo competente al quale rivolgersi. Si tratterebbe di un passo importante verso una maggiore trasparenza e presenterebbe altresì il vantaggio di diffondere maggiormente la pratica di riconoscimento, prerequisito utile per trovare un buon impiego.

Occorrerebbe un'offerta più ampia di corsi passerella e mentorato per i migranti qualificati.
Esistono pochi corsi passerella destinati agli immigrati. Peraltro, l'accento su un'integrazione rapida nel mercato del lavoro sia dei rifugiati sia delle persone disoccupate in generale è in contraddizione con le offerte passerella. Va altresì rilevato che la popolazione immigrata è raramente associata alle reti che danno accesso agli impieghi altamente qualificati. I programmi di mentorato sembrerebbero uno strumento efficace, ma la loro rilevanza e la loro entità sono tuttora limitate. A quanto pare, anche gli immigrati trarrebbero enorme beneficio da un'offerta più vasta di validazione degli apprendimenti acquisiti, dispositivo che in Svizzera si sta sviluppando gradualmente. Occorrerebbe assicurarsi che le misure di validazione degli apprendimenti acquisiti in fase di elaborazione raggiungano effettivamente gli immigrati e corrispondano alle loro esigenze. 
L'alta incidenza della

discriminazione nelle assunzioni in Svizzera meriterebbe una maggiore sensibilizzazione al problema.
Da pertinenti studi di valutazione è emersa una forte incidenza della discriminazione nelle assunzioni. Per essere convocati a un colloquio, i figli di immigrati che hanno compiuto i loro studi in Svizzera devono talvolta presentare un numero di candidature pari addirittura al quintuplo rispetto ai figli di autoctoni con un CV peraltro identico. L'incidenza relativamente debole della disoccupazione tra i figli d'immigrati induce a pensare che questi ultimi compensino la discriminazione presentando un numero superiore di candidature. L'elevato grado di discriminazione è in parte mascherato dalla condizione globalmente favorevole del mercato del lavoro; va detto inoltre che, nel dibattito pubblico, il rischio di discriminazione nelle assunzioni non viene praticamente menzionato. Tuttavia, rispetto agli autoctoni che hanno conseguito gli stessi risultati a scuola, i figli degli immigrati ci mettono il doppio di tempo per trovare un impiego. Di qui l'importanza di una maggiore sensibilizzazione al problema.

Il quadro giuridico e istituzionale della lotta alla discriminazione ̀̀ nettamente insufficiente rispetto agli altri Paesi dell'OCSE...

Il quadro giuridico e istituzionale della lotta alla discriminazione è nettamente insufficiente rispetto alla maggior parte degli altri Paesi dell'OCSE. Attualmente, la discriminazione basata sulla nazionalità non è illegale, tranne che per gli immigrati provenienti dall'UE. Inoltre, nel confronto con altri Paesi risulta che le strutture di consulenza e di orientamento sono spesso meno ben dotate e sebbene possano fornire assistenza legale, non sono autorizzate a intraprendere azioni legali. Di conseguenza, il numero di casi trattati è trascurabile se si considera il livello di discriminazione evidenziato dagli studi di valutazione.

Pertanto è importante potenziare sia il quadro giuridico sia il quadro ...e il potenziamento del quadro istituzionale della lotta alla discriminazione e portarli maggiormente a conoscenza degli immigrati. Tale riforma potrebbe essere completata da misure più incisive a favore della diversità, finalizzate alla diversificazione dei canali di reclutamento da parte dei datori di lavoro, elemento che attualmente manca in Svizzera. Il settore pubblico, nel quale gli immigrati sono sottorappresentati, deve parimenti partecipare a tale innovazione. Strumenti al servizio della diversità quali i CV anonimi, le etichette «diversità » e le consulenze in materia di diversità sono stati recentemente implementati in un certo numero di Paesi dell'OCSE. 
Il sistema d'apprendistato sembra funzionare bene per i figli degli immigrati, ma i bassi tassi di completamento della formazione meritano una maggiore attenzione.
Il Programma dell'OCSE per la valutazione internazionale delle competenze degli studenti (PISA) ha dimostrato che in Svizzera i figli d'immigrati conseguono all'età di 15 anni risultati scolastici nettamente inferiori rispetto ai figli di autoctoni e che queste differenze superano quelle osservate in molti altri Paesi dell'OCSE. L'analisi longitudinale tende a indicare che il sistema scolastico e di formazione riesce comunque a fornire successivamente qualifiche relativamente buone a questi figli d'immigrati e che nel loro caso il sistema di apprendistato sembra funzionare particolarmente bene. I figli d'immigrati hanno comunque meno probabilità di riuscire a portare a termine il loro apprendistato. Occorrerebbe studiare più a fondo tale fenomeno e adottare le corrispondenti misure correttive.

A quanto pare i figli d'immigrati hanno più problemi che in passato, per cui porvi rimedio dovrebbe essere una priorità dell'operato pubblico.

Tutti i giovani immigrati devono beneficiare della parità d'accesso alle politiche attive del mercato del lavoro destinate ai giovani.
Il meccanismo del passaggio dalla scuola all'impiego funziona bene per la maggior parte dei figli d'immigrati. Vi sono però anche segni secondo i quali i figli d'immigrati nuovamente arrivati riscontrano maggiori difficoltà che in precedenza e secondo cui molti dei figli d'immigrati nati all'estero conseguono risultati meno favorevoli. Quest'osservazione riguarda in particolar modo i figli d'immigrati i cui genitori sono originari di Paesi a basso reddito, che alcuni anni dopo aver lasciato la scuola, spesso non lavorano, non studiano e non si aggiornano (NEET). Siccome oggigiorno i figli d'immigrati provenienti da Paesi a basso reddito che accedono alla vita attiva sono numerosi, è importante i poteri pubblicio considerino prioritario migliorare i loro risultati.

Esiste una vasta gamma di misure volte a facilitare ai giovani che necessitano di un sostegno il passaggio dalla scuola all'impiego. Tuttavia, l'ammissibilità alla maggior parte delle misure di politica attiva del mercato del lavoro è spesso limitata alle persone presenti sul territorio da più di 10 anni, il che esclude numerosi giovani immigrati. Inoltre, i giovani immigrati che beneficiano di questi programmi, soprattutto del cosiddetto «semestre di motivazione », non percepiscono indennità di disoccupazione (a differenza dei giovani nati in Svizzera), e possono quindi essere tentati di entrare direttamente nel mercato del lavoro accontentandosi di un'occupazione poco qualificata. Rischiano così di essere esclusi da lavoro, studi e formazione (NEET), il che sembra accadere spesso. Assicurarsi che i figli d'immigrati e i figli di autoctoni abbiano gli stessi incentivi a partecipare ai programmi costituirebbe un primo passo per affrontare questa sfida rilevante. 
Occorre migliorare l'educazione e Secondo le ricerche effettuate in un certo numero di Paesi l'accoglienza dei bambini piccoli, dell'OCSE, il settore dell'educazione prescolare comporterebbe concentrandosi soprattutto sui figli vantaggi particolari per i figli di immigrati. Tuttavia, in Svizzera degli immigrati svantaggiati. l'educazione prescolare è attualmente largamente sottosviluppata, soprattutto per quanto riguarda i bambini dai tre ai quattro anni, un'età critica per l'integrazione. Fornire un'educazione prescolare più ampia e più mirata ai bambini di quest'età e attuare precocemente misure di sostegno linguistico avrebbe importanti vantaggi. Non ne beneficerebbero solamente i figli d'immigrati, ma anche le loro madri per le quali, rispetto alle madri autoctone, il legame tra il lavoro e il fatto di avere dei figli è molto più forte. 
DOCUMENTS DE TRAVAIL DE L'OCDE : QUESTIONS SOCIALES, EMPLOI ET MIGRATIONS

Les numéros les plus récents sont:

No. 127 THE LABOUR MARKET INTEGRATION OF IMMIGRANTS AND THEIR CHILDREN IN AUSTRIA Karolin Krause and Thomas Liebig (2011)

No. 126 ARE RECENT IMMIGRANTS DIFFERENT? A NEW PROFILE OF IMMIGRANTS IN THE OECD BASED ON DIOC 2005/06 Sarah Widmaier and Jean-Christophe Dumont (2011)

No. 125 EARNINGS VOLATILITY AND ITS CONSEQUENCES FOR HOUSEHOLDS Danielle Venn (2011)

No. 124 CRISIS, RECESSION AND THE WELFARE STATE Willem Adema, Pauline Fron and Maxime Ladaique (2011)

No. 123 AGGREGATE EARNINGS AND MACROECONOMIC SHOCKS Andrea Bassanini (2011)

No. 122 REDISTRIBUTION POLICY AND INEQUALITY REDUCTION IN OECD COUNTRIES: WHAT HAS CHANGED IN TWO DECADES? Herwig Immervoll, Linda Richardson (2011)

No. 121 OVER-QUALIFIED OR UNDER-SKILLED Glenda Quintini (2011)

No. 120 RIGHT FOR THE JOB Glenda Quintini (2011)

No.119 THE LABOUR MARKET EFFECTS OF UNEMPLOYMENT COMPENSATION IN BRAZIL Alexander Hijzen (2011)

No. 118 EARLY MATERNAL EMPLOYMENT AND CHILD DEVELOPMENT IN FIVE OECD COUNTRIES Maria del Carmen Huerta, Willem Adema, Jennifer Baxter, Miles Corak, Mette Deding, Matthew C. Gray, Wen-Jui Han, Jane Waldfogel (2011)

No. 117 WHAT DRIVES INFLOWS INTO DISABILITY?Evidence from Three OECD Countries (2011) Ana LlenaNozal and Theodora Xenogiani (disponible en anglais seulement)

No. 116 COOKING, CARING AND VOLUNTEERING: UNPAID WORK AROUND THE WORLD Veerle Miranda (2011) (disponible en anglais seulement)

No. 115 THE ROLE OF SHORT-TIME WORK SCHEMES DURING THE 2008-09 RECESSION Alexander Hijzen and Danielle Venn (2010) (disponible en anglais seulement)

No. 114 LES MIGRANTS INTERNATIONAUX DANS LES PAYS DÉVELOPPÉS, ÉMERGENTS ET EN DÉVELOPPEMENT : ÉLARGISSEMENT DU PROFIL Jean-Christophe Dumont, Gilles Spielvogel and Sarah Widmaier (2010)

No. 113 ACTIVATION POLICIES IN JAPAN

Nicola Duell, David Grubb, Shruti Singh and Peter Tergeist (2010) (disponible en anglais seulement)

No. 112 ACTIVATION POLICIES IN SWITZERLAND

Nicola Duell and Peter Tergeist with contributions from Ursula Bazant and Sylvie Cimper (2010) (disponible en anglais seulement)

No. 111 ECONOMIC DETERMINANTS AND CONSEQUENCES OF CHILD MALTREATMENT Lawrence M. Berger, Jane Waldfogel (forthcoming) (disponible en anglais seulement)

No. 110 DISTRIBUTIONAL CONSEQUENCES OF LABOR DEMAND ADJUSTMENTS TO A DOWNTURN: A MODEL-BASED APPROACH WITH APPLICATION TO GERMANY 2008-09

Herwig Immervoll, Olivier Bargain, Andreas Peichl, Sebastian Siegloch (2010) (disponible en anglais seulement) 
No. 109 DECOMPOSING NOTIONAL DEFINED-CONTRIBUTION PENSIONS: EXPERIENCE OF OECD COUNTRIES' REFORMS

Edward Whitehouse (2010) (disponible en anglais seulement)

No. 108 EARNINGS OF MEN AND WOMEN WORKING IN THE PRIVATE SECTOR: ENRICHED DATA FOR PENSIONS AND TAX-BENEFIT MODELING

Anna Cristina D'Addio and Herwig Immervoll (2010) (disponible en anglais seulement)

No. 107 INSTITUTIONAL DETERMINANTS OF WORKER FLOWS: A CROSS-COUNTRY/CROSS-INDUSTRY APPROACH

Andrea Bassanini, Andrea Garnero, Pascal Marianna, Sebastien Martin (2010) (disponible en anglais seulement)

No. 106 MONTÉE DU CHÔMAGE DES JEUNES DANS LA CRISE : COMMENT ÉVITER UN IMPACT NÉGATIF À LONG TERME SUR TOUTE UNE GÉNÉRATION ?

Stefano Scarpetta, Anne Sonnet and Thomas Manfredi (2010) (disponible en anglais seulement)

No. 105 TRENDS IN PENSION ELIGIBILITY AGES AND LIVE EXPECTANCY, 1950-2050

Rafal Chomik and Edward Whitehouse (disponible en anglais seulement)

No. 104 ISRAELI CHILD POLICY AND OUTCOMES

John Gal, Mimi Ajzenstadt, Asher Ben-Arieh, Roni Holler et Nadine Zielinsky (2010) (disponible en anglais seulement)

No. 103 REFORMING POLICIES ON FOREIGN WORKERS IN ISRAEL

Adriana Kemp (2010) (disponible en anglais seulement)

No. 102 LABOUR MARKET AND SOCIO-ECONOMIC OUTCOMES OF THE ARAB-ISRAELI POPULATION Jack Habib, Judith King, Asaf Ben Shoham, Abraham Wolde-Tsadick et Karen Lasky (2010) (disponible en anglais seulement)

No. 101 TRENDS IN SOUTH AFRICAN INCOME DISTRIBUTION AND POVERTY SINCE THE FALL OF APARTHEID

Murray Leibbrandt, Ingrid Woolard, Arden Finn et Jonathan Argent (2010)

No. 100 MINIMUM-INCOME BENEFITS IN OECD COUNTRIES: POLICY DESIGN, EFFECTIVENESS AND CHALLENGES

Herwig Immervoll (2009)

No. 99 HAPPINESS AND AGE CYCLES - RETURN TO START...? ON THE FUNCTIONAL RELATIONSHIP BETWEEN SUBJECTIVE WELL-BEING AND AGE Justina A.V. Fischer (2009)

No. 98 ACTIVATION POLICIES IN FINLAND

Nicola Duell, David Grubb et Shruti Singh (2009)

No. 97 CHILDREN OF IMMIGRANTS IN THE LABOUR MARKETS OF EU AND OECD COUNTRIES: AN OVERVIEW

Thomas Liebig et Sarah Widmaier (2009)

No. 96 INCOME DISTRIBUTION AND SUBJECTIVE HAPPINESS: A SURVEY Claudia Senik (2009)

No. 95 LOOKING INSIDE THE PERPETUAL-MOTION MACHINE: JOB AND WORKER FLOWS IN OECD COUNTRIES

Andrea Bassanini et Pascal Marianna (2009)

No. 94 JOBS FOR IMMIGRANTS: LABOUR MARKET INTEGRATION IN NORWAY

Thomas Liebig (2009)

Voir la liste complète des Documents de travail de l'OCDE sur les affaires sociales, l'emploi et les migrations : www.oecd.org/els/documentsdetravail

Parmi les autres séries de documents de travail sur le site de l'OCDE : Documents de travail de l'OCDE sur la santé 
DELSA/ELSA/WD/SEM(2012)2

\section{RECENTES PUBLICATIONS DE L'OCDE :}

LA NATURALISATION: un passeport pour une meilleure intégration des immigrés ? Librairie de l'OCDE EQUAL OPPORTUNITIES? The Labour Market Integration of the Children of Immigrants (2010) Librairie de l'OCDE OECD REVIEWS OF LABOUR MARKET AND SOCIAL POLICIES: ESTONIA (2010) www.oecd.org/els/estonia2010 DES EMPLOIS POUR LES JEUNES : GRÈCE (2010) www.oecd.org/els/emploi/jeunes DES EMPLOIS POUR LES JEUNES : DANEMARK (2010) www.oecd.org/els/emploi/jeunes OECD REVIEWS OF LABOUR MARKET AND SOCIAL POLICIES: ISRAEL (2010) www.oecd.org/els/israel2010 DES EMPLOIS POUR LES JEUNES : ÉTATS-UNIS (2009) www.oecd.org/els/emploi/jeunes DES EMPLOIS POUR LES JEUNES : POLOGNE (2009) www.oecd.org/els/emploi/jeunes PERSPECTIVES DE L'EMPLOI DE L'OCDE : Faire face à la crise de l'emploi (2009) www.oecd.org/els/emploi/perspectives

ASSURER LE BIEN-ÊTRE DES ENFANTS (2009) www.oecd.org/els/social/bienetreenfants PANORAMA DE LA SOCIÉTÉ - ÉDITION ASIE/PACIFIQUE (2009) www.oecd.org/els/social/indicators/asia OECD REVIEWS OF LABOUR MARKET AND SOCIAL POLICIES: SLOVENIA (2009) www.oecd.org/els/slovenia2009

PERSPECTIVES DES MIGRATIONS INTERNATIONALES : SOPEMI (2009) www.oecd.org/els/migrations/pmi LES PENSIONS DANS LES PAYS DE L'OCDE : Panorama des systèmes de retraites (2009) www.oecd.org/els/social/pensions/PAG-fr

DES EMPLOIS POUR LES JEUNES : FRANCE (2009) www.oecd.org/els/emploi/jeunes

PANORAMA DE LA SOCIÉTÉ 2009 - Les indicateurs sociaux de l'OCDE (2009) www.oecd.org/els/social/indicateurs/SAG

DES EMPLOIS POUR LES JEUNES : AUSTRALIE (2009) www.oecd.org/els/emploi/jeunes

OECD REVIEWS OF LABOUR MARKET AND SOCIAL POLICIES: CHILE (2009) www.oecd.org/els/chile2009 PENSIONS AT A GLANCE - SPECIAL EDITION: ASIA/PACIFIC (2009) www.oecd.org/els/social/pensions/PAG-fr MALADIE, INVALIDITÉ ET TRAVAIL : SURMONTER LES OBSTACLES (VOL. 3) - Danemark, Finlande, Irlande et Pays-Bas (2008) www.oecd.org/els/invalidite

CROISSANCE ET INÉGALITÉS : Distribution des revenues et pauvreté dans les pays de l'OCDE (2008) www.oecd.org/els/social/inegalite

DES EMPLOIS POUR LES JEUNES : JAPON (2008) www.oecd.org/els/emploi/jeunes

DES EMPLOIS POUR LES JEUNES : NORVÈGE (2008) www.oecd.org/els/emploi/jeunes

DES EMPLOIS POUR LES JEUNES : ROYAUME-UNI (2008) www.oecd.org/els/emploi/jeunes

DES EMPLOIS POUR LES JEUNES : CANADA (2008) www.oecd.org/els/emploi/jeunes

Pour une liste complète, veuillez consulter la librairie en ligne de l'OCDE à www.oecd.org/bookshop 